LATE-TYPE Ca II EMISSION-LINE STARS IN THE SOUTHERN HEMISPHERE

\author{
A thesis \\ submitted in partial fulfilment \\ of the requirements for the Degree \\ of \\ Doctor of Philosophy \\ at the \\ University of Canterbury \\ by \\ A.C. Collier
}

University of Canterbury 
The results of a photometric and spectroscopic study of twenty-three late-type southern stars with strong Ca II $\mathrm{H}$ and $\mathrm{K}$ emission are presented.

The presence of photometric variability, with similar properties to the photometric wave phenomenon seen in the RS Canum Venaticorum binaries, is noted in thirteen of the fifteen stars for which extensive photometry was obtained. of twenty stars for which high-resolution radial velocity measurements were obtained, ten are found to be single-lined and seven are found to be double-lined spectroscopic binaries. orbital periods are determined for eight previously-unknown binaries. The periods range from 0.66 up to 53.9 days. Two stars with no radial velocity variations but with high rotational broadening ( $v_{e} \sin i \geqslant 40 \mathrm{~km} \mathrm{~s}^{-1}$ ) of their spectra are identified as probable new members of the FK Comae group of rapidly-rotating, chromospherically-active late-type giants.

High resolution $(\Delta \lambda<0.3 \AA) \mathrm{H} \alpha$ spectra were obtained of twenty-one of the candidate stars. Nine stars show $\mathrm{H} \alpha$ as an emission feature above continuum level. Eight show $\mathrm{H} \alpha$ either absent or unusually shallow, due to filling of the absorption profile with emission. High resolution $(\Delta \lambda<0.3 \AA)$ $\mathrm{Ca}$ II $\mathrm{H}$ and $\mathrm{K}$ spectra of ten of the program stars yield line surface fluxes in the range

$$
1.6 \times 10^{6} \leqslant F\left(K_{1}\right)+F\left(H_{1}\right) \leqslant 1.9 \times 10^{7} \text { erg } \mathrm{cm}^{-2} \mathrm{~s}^{-1}
$$

in the $\mathrm{H}$ and $\mathrm{K}$ emission cores. 
The high proportion of single-lined RS CVn binaries in the sample is discussed in terms of selection effects. The space density of RS CVn systems with mass ratios less than $q \simeq 0.8$ is found to be at least equal to that of the $q \geqslant 0.8$ systems. This implies a total space density of at least $10^{-5} \mathrm{pc}^{-3}$ for the RS CVn binaries.

Differences between the RS CVn binaries and the related short-period, long-period and semi-detached $\mathrm{G}$ and $\mathrm{K}$ binaries with $\mathrm{Ca}$ II $\mathrm{H}$ and $\mathrm{K}$ emission are discussed in terms of evolutionary processes in close binary systems. A space density calculation based on the evolutionary requirements for membership of the RS CVn group is shown to reproduce closely the mass and luminosity distributions among the RS CVn binaries. The theoretical space density of RS CVn systems in the Galactic plane is found to be $1.9 \times 10^{-5} \mathrm{pc}^{-3}$ by this method, in reasonable agreement with the observed value. 
CONTENTS

PREFACE

1. INTRODUCTION AND SOURCE SELECTION

1.1 The RS CVn Binaries. 2

1.2 Evolutionary status. 5

1.3 Activity Cycles and Period-Activity 7 Relations.

1.4 Evidence for Stellar Dynamos in Main Sequence Stars.

1.5 Theoretical Dynamo Models.

1.6 Applications to RS CVn Systems.

1.7 Source selection.

2 PHOTOMETRIC SURVEY 15

2.1 Introduction. 15

2.2 Equipment. 16

2.3 Observing Technique and Data Reduction. 17

2.4 Results 20

2.4.1 Extinction. $\quad 20$

2.4.2 Instrumental Response. 21

2.4.3 Magnitudes and Colours of Program 22 and Comparison Stars.

2.4.4 Variability and Rotation Period 26 Analysis.

2.4.5 Starspot Size and Temperature. 


\section{HIGH RESOLUTION SPECTROSCOPY}

3.1 Introduction.

3.2 Instrumentation and observational

Techniques

3.2.1 Mg I b Coudé spectra.

3.2.2 Ha Coudé Échelle Spectra.

3.2.3 Ca II $\mathrm{H}$ and $\mathrm{K}$ Coudé Spectra.

3.2.4 Ca II K Cassegrain Échelle Spectra.

3.2.5 UBV Photometry.

3.3 Data Reduction: Mg I b Spectra:

3.3.1 Preliminary Treatment.

3.3.2 Wavelength Calibration.

3.3.3 Radial Velocity Reductions.

3.4 Data Reduction: Ha Spectrá

3.4.1 Preliminary Treatment.

3.4.2 Wavelength Calibration.

3.4.3 Velocity Reductions.

3.4.4 Continuum Fitting.

3.5 Data Reduction: Ca II H and $\mathrm{K}$ Spectrá

3.5.1 Preliminary Treatment.

3.5.2 Wavelength Calibration.

3.5.3 Velocity Reductions.

3.5.4 Surface Gravities Using the Wilson-Bappu Effect.

3.5.5 Ca II $H$ and $K$ Line Surface Fluxes.

3.5.6 Measurement of Ca II Emission Flux Variations.

3.6 Data Reduction: UBV Photometry at MSO. 71

3.7 Preliminary Radial Velocity Solutions. 71 
4 RESULTS FOR INDIVIDUAL SYSTEMS

4.1 HD 5303: 73

4.1.1 Radial Velocity Observations. $\quad 73$

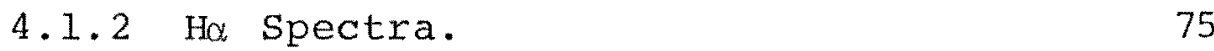

4.1.3 Further Photometric Analysis. 79

4.2 HD $8435 \quad 87$

4.2.1 Radial Velocity Observations. 87

4.2.2 H $\alpha$ Spectra. $\quad 94$

4.2.3 Photometric Observations. 94

4.2.4 Summary. $\quad 96$

$\begin{array}{lll}4.3 & H D & 14643\end{array}$

4.3.1 Radial Velocity Observations. 97

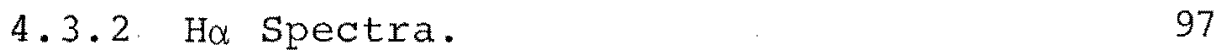

4.3.3 Photometric Observations. 100

$\begin{array}{lll}4.4 & \text { HD } 32918 & 101\end{array}$

4.4.1 Colour Changes and Spot Temperature. 101

$\begin{array}{lll}4.5 & H D & 106\end{array}$

4.5.1 Radial Velocity Observations. 106

4.5.2 Photometric Observations. 106

4.6. HD $36705 \quad 108$

4.6.1 Photometric Observations. 109

$\begin{array}{llll}4.7 & H D & 37434 & 111\end{array}$

4.7.1 Radial Velocity Observations. 111

4.7.2 Ha Spectra. 111 
4 Cont....

4.8 HD 37847

4.8.1 H $\alpha$ Spectra.

4.9 HD 39937

4.9.1 Radial Velocity Observations.

4.10 HD 42504

4.10.1 $\mathrm{H} \alpha$ Spectra.

4.11.1 Radial Velocity Observations.

4.11.2 $\mathrm{H} \alpha$ Spectra.

4.11.3 Ca II H and $\mathrm{K}$ Spectra.

119

4.11.4 Discussion.

4.12 HD 81410

4.12.1 Ha Spectra.

4.12.2 Ca II $\mathrm{H}$ and $\mathrm{K}$ spectra.

4.12.3 Photometric observations. 129

4.12.4 Discussion.

4.13.1 Ha Spectra.

4.13.2 Ca II $\mathrm{H}$ and $\mathrm{K}$ spectra.

4.13.3 Photometric Observations.

4.13.4 Discussion 
4 cont....

4.14 HD $101379=$ HR $4492 \quad 142$

4.14.1 Ha Spectra. $\quad 142$

4.14.2 Ca II $\mathrm{H}$ and $\mathrm{K}$ Spectra. 144

4.14.3 Photometric Observations and Analysis.

4.14.4 Ca II Absolute Surface Flux Calibration.

4.15 HD 119285

4.15.1 Ha Spectra. 156

4.15.2 Ca II $\mathrm{H}$ and $\mathrm{K}$ Spectra. 156

4.16 HD $127535 \quad 161$

4.16.1 Ha spectra. 161

4.16.2 Ca II H and $\mathrm{K}$ Spectra. 163

4.16.3 Photometric Observations. 167

4.16.4 Discussion. 167

$\begin{array}{llll}4.17 & H D & 137164 & 169\end{array}$

4.17.I $\mathrm{H} \alpha$ Spectra. 169

4.17.2 Ca II $\mathrm{H}$ and $\mathrm{K}$ spectra. 169

4.17.3 Photometric Observations. 170

4.17.4 Discussion. $\quad .174$

4.18 HD $155555 \quad 175$

4.18.1 Photometric Observations. 175

4.18.2 H $\alpha$ Spectra. 175

4.18.3 Ca II $H$ and $K$ Spectra. 176 
4 Cont....

$\begin{array}{lll}4.19 & \text { HD } 165141 & 179\end{array}$

4.19.1 Ho Spectra. $\quad 179$

4.19.2 Ca II $\mathrm{H}$ and $\mathrm{K}$ Spectra. 179

4.20 HD $181809-180$

4.20.1 Photometric Observations. 180

4.20.2 Ha Spectra. 181

4.20.3 Ca II $\mathrm{H}$ and $\mathrm{K}$ Spectra. 181

$\begin{array}{lll}4.21 & \text { HD } 185510 & 184\end{array}$

4.21.1 Photometric Observations. 184

4.22 HD $195040 \quad 184$

4.22.1 Radial Velocity Observations. 184

4.22.2 Ha Spectra. 185

4.22.3 Photometric Observations. 185

$\begin{array}{lll}4.23 & \text { HD } 196818 & 190\end{array}$

4.23.1 Photometric Observations. 190

4.23.2 Radial Velocity Observations. $\quad 190$

4.23.3 Ha Spectra. 191

5 SUMMARY AND DISCUSSION 192

5.1 Summary of Results 192

5.1.1 Duplicity. 192

5.1.2 Orbital Periods. 192

5.1.3 Photometric Variability. 193 
5 Cont...

5.1.4 Ca II $\mathrm{H}$ and $\mathrm{K}$ Emission. 194

5.1.5 H $\alpha$ Emission.

5.2 Selection Effects

5.2.1 Observed Sample Properties for Different Detection Methods. 201

5.2.2 Effects of Secondary Luminosity on Detection Likelihood.

5.3 Evolutionary Constraints on the observed Characteristics of RS CVn Systems 210

5.3.1 Distribution of Initial Mass Ratios.

5.3.2 Necessary Conditions for the RS CVn Syndrome.

5.3.3 Period Constraints on RS CVn Binary systems.

5.4 Main Sequence Systems.

5.5 Semi-Detached systems.

5.6 Giant systems.

5.7 Observed and Theoretical Mass and Luminosity Functions for RS CVn systems. 
REFERENCES
APPENDIX I : "Discovery of Two FK Comae stars in the Southern Hemisphere". (Collier, 1982).

APPENDIX II : "A Coordinated Radio and $\mathrm{H} \alpha$ Survey of Southern RS CVn Systems and Related Objects". (Collier, Haynes, Slee, Wright and Hillier, 1982). 
$2.1 \quad$ Mt. John Extinction Coefficients

2.2 Mt. John Photometric System Transformation

Coefficients.

2.3 Magnitudes and Colour Indices of Program Stars.

2.4 Photometrically-determined Rotation Periods for Four Stars.

3.1 Radial Velocities of standard stars.

Stars Used for Calibration of Empirical Wilson-Bappu Relation.

3.3 Ca II $K$ Emission Widths and Surface Gravities for Nine Stars.

Absolute $\mathrm{Ca}$ II $\mathrm{H}$ and $\mathrm{K}$ Emission Surface Fluxes for Ten stars.

3.5 Relative Ca II K Emission Core Flux Variations for Eight stars.

3.6 Preliminary Radial Velocity Solutions for Six Stars.

4.1.1 Radial Velocities of HD 5303.

4.1.2 Photometric Parameters adopted for HD 5303

WINK Lightcurve Synthesis.

4.2.1 Radial Velocities of HD 8435 .

4.3.1 Radial Velocities of HD 14643 .

4.7.1 Radial Velocities of HD 37434 .

4.8.1 Radial Velocities of HD 37847 .

4.9.1 Radial Velocities of HD 39937.

4.10.1 Radial Velocities of HD 42504 . 
4.11.1 Radial Velocities of HD 46697.

4.12.1 Radial Velocities of HD 81410. 126

4.13.1 Radial Velocities of HD $101309 . \quad 134$

4.14.1 Radial Velocities of HD $101379=\mathrm{HR} 4492 . \quad 146$

4.14.2 Model Parameters for Various Primary Masses and Orbital Inclinations in HD 101379.

4.15.1 Radial Velocities of HD 119285.

4.16.1 Radial Velocities of HD 127535. 164

4.17.1 Radial Velocities of HD 137164.

4.19.1 Radial Velocities of HD 165141. 180

4.20.1 Radial Velocities of HD 181809.

4.22.1 Radial Velocities of HD 195040. 189

4.22.2 Best-Fit Radial Velocity Solution for HD 195040.

5.2.1 Effects of Various Secondary Masses on Observable Parameters of a Model RS CVn System.

5.2.2 Variation of Model System Colour Index with Secondary Mass.

5.3.1 Limiting Orbital Periods on Synchronised, Detached RS CVn Systems.

5.3.2 Limiting Orbital Periods for Case B Evolution and Proportion of RS CVn Binaries for Various Masses.

5.7.1 Theoretical Mass and Luminosity Functions for Convective Hertzsprung Gap Stars.

5.7.2 Predicted and Observed Numbers of RS CVn Systems as a Function of Apparent Visual 


\section{LIST OF FIGURES}

FIGURE

PAGE

$2.1 \quad\left(V-R_{C}\right)$ and $(V-I)_{C}$ Vs. $(B-V)$ for Program,

Comparison and standard stars.

2.2 Photometric Periodograms for Four Program

Stars.

3.1 Empirical Wilson-Bappu Relation Calibration.

3.2 Schematic Representation of Ca II K Relative

Flux Measurements.

3.3 Variations in Ca II $K$ Relative Flux for

Eight Stars.

4.1.1 Cross-Correlation Function of HD 5303

Mg I b spèctrum.

4.1.2 Radial Velocities of HD 5303.

4.1 .

Ho Spectra of HD 5303.

4.1.4 WINK Synthesised Lightcurve of HD 5303.

$4.1 \cdot 5$

(O-C) Lightcurves of HD 5303.

4.2 .1

c.c.f.s of HD $8435 \mathrm{Mg}$ I b spectra.

4.2.2 Primary Radial Velocity vs. sin $\varnothing$ for HD 8435 .

Ho Spectra of HD 14643.

4.3.2 Periodogram for HD 14643 Radial Velocities.

4.4.1 Variations of Colour Indices with $V$

Magnitude in HD 32918.

4.5.1 V Lightcurve of HD 34802 .

4.6.1 V Lightcurve of HD 36705.

4.7 .1

Ho Spectrum of HD 37434 . 
4.9.1 Ha Spectrum of HD 39937.

4.10.1 H $\alpha$ Spectrum of HD 42504.

4.11.1 H H Spectra of HD 46697.

4.11.2 Periodogram for HD 46697 Radial Velocities.

4.11.3. Radial Velocities of HD 46697.

4.12.1 Ho Spectra of HD 81410.

4.12.2 Periodogram for HD 81410 Radial Velocities.

4.12.3 Radial Velocities, V Lightcurve and Ca II $\mathrm{K}$ Fluxes of HD 81410 .

4.12.4 V Lightcurve of HD 81410 from Eggen's (1973) Photometry.

4.13.1 Ha Spectra of HD 101309.

4.13.2: Ca II K Profile of HD 101309.

4.13.3 Periodogram for HD 101309 Radial Velocities.

4.13.4 Radial Velocities, V Lightcurve and Ca II K Fluxes of HD 101309.

4.14.1 Ca II $\mathrm{H}$ and $\mathrm{K}$ Spectrum of $\mathrm{HD} 101379=\mathrm{HR} 4492.145$

4.14.2 Periodogram for HR 4492 Radial Velocities. 148

4.14.3 Radial Velocities and V Lightcurve of

HR 4492 .

4.15.1 H H Spectra of HD 119285.

4.15.2 Periodogram for HD 119285 Radial Velocities.

4.15.3 Radial Velocities of HD 119285.

4.16.1 Ha Spectra of HD 127535.

4.16.2 Periodogram for HD 127535 Radial Velocities.

4.16.3 Radial Velocities, V Lightcurve and Ca II $\mathrm{K}$ Fluxes of HD 127535 . 
4.17.1 Ha Spectra of HD 137164.

4.17.2 Radial Velocities and $V$ Lightcurve of HD 137164.

4.18.1 V Lightcurve of HD 155555.

4.18.2 Ho spectra of HD 155555.

4.20.1 Ha Spectrum of HD 181809.

4.21.1 V Lightcurve of HD 185510.

4.22.1 Periodograms for HD 195040 Radial Velocities and Photometry.

4.22.2 Radial Velocities and V Lightcurve of HD 195040 .

4.23.1 Ho spectrum of HD 196818.

5.1.1 Ca II $H$ and $K$ spectra for Eight stars.

5.1.2 Ca II $H$ and $K$ surface Flux vs. Orbital Period for Eight Stars.

5.2.1 Isochrone for $t=4 \times 10^{9}$ years, from Sandage and Eggen (1969).

5.2.2 Observable Parameters vs. Mass Ratio for Model RS CVn System.

5.2.3 Deviations of Model System Colour Indices from Mean Relations for Various Secondary

Masses.

5.7.1 Theoretical Mass Function for Convective Hertzsprung Gap Stars, and Observed Distribution of RS CVn Primary Masses.

5.7.2 Theoretical Mass-Luminosity Relation for Stars at Base of Giant Branch.

5.7.3 Theoretical Luminosity Functions for Late Hertzsprung Gap Stars and RS CVn Primaries. 
5.7.4 Theoretical Absolute Magnitude

Distribution for RS CVn primaries

brighter than $\mathrm{V}=8.0$ and $\mathrm{V}=7.0$. 
PREFACE

The purpose of the programme of research described in this thesis was to investigate the natures of a number of bright $(V<9.0)$, late-type southern stars whose spectra exhibit strong, narrow emission features in the cores of the $\mathrm{H}$ and $\mathrm{K}$ resonance $\mathrm{lines}$ of singly-ionised calcium.

The theories of mechanisms by which such emission is produced in late-type stars are reviewed in chapter 1. Particular attention is paid to the peculiar RS Canum Venaticorum binary stars, which are among the strongest known Ca II emitters.

The equipment, observational techniques and data reduction methods employed in this investigation of southern Ca II emitting stars are described in Chapters 2 and 3. The results obtained for each of these 23 stars are presented in Chapter 4 .

The properties of the stars in the sample were found to differ in some ways from those obtained for similar objects in the northern hemisphere. These differences are discussed in Chapter 5, in terms of selection effects pertaining to different survey methods. The evolutionary theory for the origin of the RS CVn binaries is also tested against observation in this chapter.

Two papers incorporating the results of part of this work are to be published shortly, and are included in this thesis as appendices. 
CHAPTER 1

\section{INTRODUCTION AND SOURCE SELECTION}

\subsection{THE RS CVN BINARIES}

The RS Canum Venaticorum (RS CVn) binaries are a class of close binary systems containing late-type components displaying high levels of surface, chromospheric and coronal activity which appear to be magnetic in origin.

Although the requirements for membership of the class have in the past been somewhat vague, Hall (1976) has proposed a working definition of an RS CVn binary as one with the hotter component having an F-G V - IV spectral type, an orbital period between one day and two weeks, and strong $\mathrm{Ca}$ II $\mathrm{H}$ and $\mathrm{K}$ emission visible outside eclipse in the spectrum of the cooler component or, in some cases, in both components.

Related groups of detached main sequence $G$ binaries with periods less than one day, and of binaries with longer periods than the RS CVn systems and containing $k$ type components of luminosity class III also exist. These also show the strong $\mathrm{Ca}$ II emission features.

The RS CVn binaries initially attracted attention as a class of eclipsing binaries, because of their peculiar lightcurves.

Sitterly (1930) noted that the primary eclipse of RS CVn itself was asymmetric, the light levels immediately before and after primary eclipse differing by 0.13 magnitude. Sitterly offered two possible explanations for the lightcurve distortion. One involved a compact "hot spot" on one hemisphere of the hotter star; the other required a tidally- 
elongated ellipsoidal configuration for both stars, with a tidal lag of about $45^{\circ}$.

Sitterly's photometric solution indicated that the cool secondary's radius and temperature were such that it occupied a position on the Hextzsprung-Russell diagram midway between the main sequence and the giant branch. Joy's (1930) spectroscopic study of the system supported this conclusion.

Kron (1947) observed a similar, quasi-sinusoidal distortion of the lightcurve of the eclipsing binary AR Lacertae. He proposed a model in which the variation was caused by a longitudinally-asymmetric distribution of large, cool starspots on the surface of one or both co-rotating binary components.

Photoelectric lightcurves of RS CVn obtained by Keller and Limber (1951), Popper (1961), Chisari and Lacona (1965) and Catalano and Rodonò $(1967,1969)$ revealed that the lightcurve distortion was not static, but varied in both its mean level and amplitude. The phase of the wave minimum tended to migrate towards decreasing orbital phase on a timescale of several years, while the wave amplitude varied between 0.05 and 0.20 magnitudes.

Popper (1961) noted that the colour changes accompanying the changes in the mean lightcurve level corresponded to the colour of the cooler star, and suggested that some form of pulsation of the cool star might be responsible for the variations. He later extended this idea (Popper, 1977), proposing that the phenomenon was due to a non-radial standing wave in the cool star's outer envelope.

Catalano and Rodonò $(1967,1969,1974)$ developed a model in which the variation was cuased by a ring of circumstellar material, tilted with respect to the orbital plane of the system, and with a longitudinally-asymmetric optical 
cross section.

Hall (1972), Eaton and Hall (1979) and Shore and Hall

(1980) have developed the starspot model further. Eaton and Hall attempted to fit model lightcurves based on the three types of model to observations of RS CVn. Only the cool spot model gave a satisfactory fit to the observations during secondary eclipse.

The unusually strong $\mathrm{Ca}$ II $\mathrm{H}$ and $\mathrm{K}$ emission features associated with the cool components of RS CVn systems appear to be chromospheric in origin. Ultraviolet spectra of these stars taken with the COPERNICUS and International Ultraviolet Explorer (IUE) satellites have revealed rich emission spectra of the type associated with solar active regions. The line surface fluxes in these regions are in some cases two orders of magnitude greater than those observed in the spectrum of the quiet Sun (Simon and Linsky, 1980).

Erratically variable $\mathrm{H} \alpha$ emission has also been observed in several of the most active RS CVn systems, again apparently associated with the cooler, active component. This emission appears to originate in surface flaring (Bopp and Talcott, 1980). This hypothesis is supported by the fact that the RS CVn systems form an important class of flaring, non-thermal stellar radio sources (Spangler, Owen and Hulse, 1976). A prolonged radio flaring episode in the HR 1099 system during 1978 February was extensively observed at radio (Feldman et.al., 1978), optical (Fraquelli, 1978; Hearnshaw, 1978; Bopp and Talcott, 1978) and ultraviolet (Weiler et.al., 1978) wavelengths. Enhanced $\mathrm{H} \alpha$ and ultraviolet emission was observed during this flare event. The detection of many of the RS CVn systems as 
flaring soft X-ray sources (Walter, Charles and Bowyer, 1978; Walter et. al., 1980) indicates that these stars have solar-like coronae, but with higher temperatures and densities.

\section{I.2 EVOLUTIONARY STATUS}

In an extensive review of the properties of the RS CVn systems, Hall (1976) noted that in nearly all of these binaries, the active star was a $\mathrm{KO} / 1$ subgiant of 1.0 to 1.5 solar masses, while the hotter component was usually slightly less massive.

The evolutionary status of these objects has been much discussed. Hall (1972) suggested that the subgiant in RS CVn itself might be a pre-main-sequence object similar to the T Tauri stars.

Montle (1973) derived a kinematical age of 1 to $3 \times 10^{8}$ years for the 25 eclipsing RS CVn systems known at that time. This value was based on the mean observed height of these systems above and below the Galactic plane, and on their velocity dispersion. Using a revised age-velocity dispersion calibration by wielen (1974), Hall obtained a kinematical age of about $2 \times 10^{9}$ years. This, combined with the known presence of low-mass, main-sequence visual companions to several RS CVn systems, suggested that the timescales involved were not consistent with a pre-main-sequence evolutionary status. Biermann and Hall (1976) examined several scenarios which might lead to the formation of RS CVn systems. They concluded on the strength of the evidence cited above that a post-main-sequence interpretation was more likely. However, they noted that the RS CVn binaries did not appear to fit in with the post-main-sequence mass transfer theory for close 
binaries, and that their observed space density was too high for this explanation to be likely. Instead, they suggested that the RS CVn binaries might represent the end-products of post-main-sequence fission of rapidly-rotating single main sequence stars.

Popper and Ulrich (1977) examined the colour-luminosity, mass-radius and mass-colour relations for 23 eclipsing and 3 non-eclipsing RS CVn systems. They found that the results were consistent with an evolutionary picture in which the more massive component of a former main-sequence binary system had evolved off the main sequence toward the base of the ascending giant branch on its Hayashi track.

The main objection to this interpretation was that at least two RS CVn systems, SS Bootis and UX Cornae, contained subgiant components of one solar mass or slightly less. Such stars should not have evolved off the main sequence during the lifetime of the Galaxy.

However, period changes have been observed in most of the RS CVn systems, providing direct evidence that a certain amount of secular mass loss is occurring in these systems. Hall, Kreiner and Shore (1980) used a model in which a convectively-driven stellar wind corolates with the active star out to the Alfven radius, in order to estimate the mass loss rates required to produce the observed period changes in 34 eclipsing $R S C V n$ and related systems. In the cases of SS Boo and UX Com, mass loss rates of between $10^{-7}$ and $10^{-8}$ $M_{\odot}$ per year were indicated. Such rates would result in a significant reduction in mass on the timescale (about $10^{8}$ years) required for a star of between 1.0 and $1.5 \mathrm{M}_{\odot}$ to complete its Hertzsprung gap evolution.

The post-main-sequence binary hypothesis offers the most self-consistent evolutionary picture, and is found in 
Chapter 5 to give a theoretical space density for the RS CVn systems which is close to the observed value.

However, there may be exceptions to the rule, particularly as the RS CVn systems and their related groups show a wide range of general characteristics within Hall's (1976) working definition. Although most of the eclipsing and double-lined RS CVn systems are detached and have mass ratios close to unity, the group also includes several semidetached systems (e.g. RT Lac, HR 5110) which appear to be products of post-main-sequence mass exchange. It seems that the RS CVn phenomenon may occur in stars with considerably different structures and evolutionary histories.

1.3 ACTIVITY CYCLES AND PERIOD-ACTIVITY RELATIONS

Observations of several northern RS CVn systems over several decades have revealed the presence of cyclic activity, with periods of the order of 20-30 years for RS CVn and RT Lac. In these systems, the variation in the total number of spots as the cycle progresses, apparently causes the mean luminosity of $t$. spotted star to vary with the cycle period.

Hall (1972) hypothesised that the migration of the photometric wave towards decreasing orbital phase was due to differential rotation in the convective envelope of the active star. The migration rate varies cyclically with the mean level of starspot activity in $\mathrm{RS} C V \mathrm{~V}$, the migration rate having its greatest value immediately following the starspot minimum in the activity cycle.

Shore and Hall (1980) have applied a simple model of the solar cycle developed by Babcock (1961) to these cyclic phenomena in RS CVn. They find that the observations are consistent with a solar-like drift of the active region 
towards the stellar equator as the cycle progresses.

It thus appears that the mechanism which gives rise to the surface activity in the RS CVn stars is of a similar nature to the hydromagnetic dynamo process responsible for the cyclic surface activity observed in the sun. Such a dynamo process is dependent to a large extent on the axial rotation rate of the star, and recent X-ray observations have confirmed that a correlation between magnetic field strengths in the coronae of $\mathrm{RS} C \mathrm{CV}$ systems and their rotation rates appears to exist.

Walter and Bowyer (1981) have analysed X-ray observations of a nearly complete sample of RS CVn systems. Their finding is that the ratio of the soft $x$-ray Iuminosity to the bolometric luminosity of an RS CVn system is directly proportional to the rotational angular velocity $\Omega$ of the system, over a range of $\Omega$ spanning two orders of magnitude.

As the soft $x$-ray emission is thought to originate in magnetically-contained plasma within coronal loops (Walter, 1980; Rosner, Tucker and Vaiana, 1978) the soft X-ray luminosity is presumed to be closely related to the extent of the star's magnetic field. The correlation between $\mathrm{X}$-ray luminosity and rotation rate thus provides further evidence for a rotational1y-dependent dynamo mechanism in these stars.

1.4 EVIDENCE FOR STELLAR DYNAMOS IN MAIN SEQUENCE STARS

The existence of solar-like activity in single, lower main sequence stars has been established from the results of a long-running $\mathrm{Ca}$ II $\mathrm{H}$ and $\mathrm{K}$ flux measurement programme initiated by O.C. Wilson in 1966 (Wilson, 1968).

The association of regions of enhanced chromospheric emission (plages) with active magnetic regions on the sun's 
surface is well-established. The cyclic Ca II flux variations are interpreted, by analogy with the solar observations, as being due to variations in the fraction of the stellar surface covered with active magnetic regions. This suggests that magnetic activity cycles are a common feature in lower main sequence stars.

Moreover, the Wilson data in many cases show shorter term periodicities which have been interpreted as a rotational modulation of the emission flux (Vaughan et.al., 1981). Such a modulation is presumably due to a non-uniform longitudinal distribution of active regions on the stellar surface, such as is frequently observed on the Sun. As a result, Stimets and Giles (1980) and Vaughan et.al., (1981) have for the first time succeeded in measuring directly the axial rotation periods of a number of single, lower main sequence stars. Vaughan et.al. found that for stars of a given spectral type, the mean $\mathrm{Ca}$ II $\mathrm{H}$ and $\mathrm{K}$ emission flux was tightly correlated with the axial rotation rate. The relationship varies considerably according to the spectral type of the star, the emission being stronger for stars of later spectral type.

This is partly a contrast effect. The wilson flux index is defined as the ratio of the flux in two $1 \AA$ bands centred on the $\mathrm{H}$ and $\mathrm{K}$ lines, to the photospheric flux in two $20 \AA$ bands centred at $3901.068 \AA$ and $4001.067 \AA$. At these wavelengths, the photospheric flux increases rapidly with increasing effective temperature. However, this effect does not account completely for the temperature dependence of the emission flux. 
Recent theoretical models of dynamo activity in lower main sequence stars have been developed and related to the observations of Vaughan et.al. (1981) by Durney, Mihalas and Robinson (1981), Durney and Robinson (1982) and Robinson and Durney (1982).

These models are based on the $\alpha \omega$ dynamo process initially postulated by Parker (1955) as a likely hydromagnetic mechanism for producing self-sustaining magnetic fields in the convective envelopes of differentially-rotating stars. The details of this process have been developed further by Steenbeck and Krause (1969), Leighton (1969) and Yoshimura (1975). Models using this process have been applied with some success in reproducing the solar magnetic cycle (e.g. Yoshimura, 1975) although these models are largely dependent on the assumed form of the fluid motion within the convective zone, which is not well understood.

The usual forms of the dynamo equations for the $\alpha w$ process (e.g. Yoshimura, 1975) are

$$
\begin{aligned}
& \frac{\partial \mathrm{A}}{\partial t}=\alpha \mathrm{B}_{\phi}+\eta \nabla^{2} \mathrm{~A} \\
& \frac{\partial \mathrm{B}_{\phi}}{\partial \mathrm{t}}=\nabla \Omega+\nabla(\mathrm{Ar} \sin \theta)+\eta \nabla^{2} \mathrm{~B}_{\phi} \\
& \underset{\sim \mathrm{B}}{\mathrm{B}}=\nabla \times(0,0, \mathrm{~A}) .
\end{aligned}
$$

In a model of this type, an initial poloidal magnetic field $\underset{\sim p}{\mathrm{~B}}$ is acted upon by fluid shear due to the differential rotation of the plasma within the convective envelope, to produce a toroidal field component $\mathrm{B}_{\phi}$ within the plasma. This effect is represented by the first term in Eq. (1-1 b), in which $\theta$ is the polar angle. The poloidal field is regenerated 
from the toroidal component due to the effect of turbulent cyclonic convection, as expressed in the first term of Eq. (1-1 a). The quantity $\alpha$ is defined by steenbeck and Krause (1969) as

$$
\alpha=-\frac{1}{3} \tau<\underset{\sim}{v} \cdot \nabla \times \underset{\sim}{v}>
$$

where $\mathrm{V}$ describes the turbulent fluid motion and $\tau$ is the lifetime of the turbulent eddies. Dissipation is accounted for in Eqs. (1-1 a) and $(1-1 b)$, where $n$ is the turbulent magnetic diffusivity.

As field amplification proceeds within the convective envelope, local field concentrations formed by small-scale irregularities in the fluid motion give rise to regions of increased magnetic viscosity. These interact with the differential rotation to produce twisted "ropes" or tubes of magnetic flux. Parker (1975) found that the requirement of internal and external pressure equilibrium in these tubes led to buoyant instability if the magnetic pressure within the tube become comparable with the external gas pressure. This effectively limits the field strength attainable by the aw process and, when buoyant eruption of the flux tubes takes place, results in the formation of magnetic surface features such as sunspots and plages. Parker found that the buoyant rise velocity of the flux tubes decreased with depth at constant magnetic field strength. As a consequence, the aw dynamo is effectively limited to the base of the convective zone.

Durney and Robinson assumed accordingly that the surface magnetic field was essentially dependent on the local conditions at the base of the convective zone. These in turn determined the field strength attainable before the flux 
tubes rose out of the amplification region. Their local solutions to Eqs.(1-1) produced surface magnetic field strengths and areas whose dependences on rotation rate and spectral type are remarkably similar to the temperature rotation - Ca II flux relations found by Vaughan et.a1.(1981)

A further development of this localised model (Robinson and Durney, 1982) has had some success in predicting the activity cycle periods in three stars for which both cycle period and axial rotation period have been measured.

1.6 APPLICATIONS TO RS CVN SYSTEMS

The results of these observational and theoretical investigations of period-activity relations in main sequence stars provide several important insights into the RS CVn syndrome.

Clearly, the most important factors contributing to the activity levels observed in the RS CVn stars will be the nature of the differential rotation within the convective envelope, the rotation rate, and the inner radius, density, temperature and scale height at the base of the convective envelope (cf. Durney and Robinson, 1982).

All of these except the differential rotation may be inferred from model interior calculations and direct observation. Any attempt to model the RS CVn dynamo mechanism in detail must therefore await the development of models for the differential rotation in the convective envelope of a tidally-synchronised binary component.

Shore and Hall (1980) present observational evidence based on the variations in starspot migration rate, that the surface differential rotation rate $\frac{\Delta \Omega}{\Omega_{0}}$ in the active component of RS CVn itself is some two orders of magnitude lower than 
that in the sun, where the surface differential rotation is assumed to depend on stellar latitude according to

$$
\Omega(\beta)=\Omega_{0}-\Delta \Omega \sin ^{2} \beta
$$

Whether such a low differential rotation rate is a general feature of the deep convective envelopes in late Hertzsprung gap stars or an artifact of the tidal forces operating in a synchronously rotating binary component can only be resolved by future theoretical studies.

Any theoretical study of the dynamo mechanism in the RS CVn binaries must be related to a thorough knowledge of their observational properties. A great many observations must be made in order to determine the dependences of activity levels and cycle periods on such physical parameters as rotation rates, temperatures, masses and radii of active RS CVn components.

One of the most important questions which must be answered is whether the presence of a close (but detached) binary companion has a significant effect on the evolutionary processes which determine a star's interior structure. The effects of rapid axial rotation on stellar evolution are not well understood. It seems likely that even in detached systems such as the RS CVn stars, the rate of secular mass loss is enhanced through increased coronal activity due to rapid rotation. To some extent, the importance of rotationally. linked evolutionary effects can be assessed by comparing theoretical space densities and luminosity distributions calculated using non-rotating single-star evolutionary models, with the observed distribution of RS CVn binaries. It is part of the aim of the work described in this thesis to attempt such a comparison. 
$1.7 \quad$ SOURCE SELECTION

The primary aim of the observations presented in this thesis was to locate and study new RS CVn systems in the southern hemisphere, where little research has hitherto been carried out in this field.

The RS CVn systems in the northern hemisphere are predominantly eclipsing binaries, a selection effect which is due to the fact that these systems originally attracted attention because of their photometric peculiarities outside eclipse.

The candidate stars for this project were drawn from the lists of southern RS CVn candidates of Hearnshaw (1979) and Weiler and stencel (1980).

Two known but little-studied RS CVn systems which were selected for photometric observation were HD 5303 (Hearnshaw and Oliver, 1977) and HD 155555 (Bennett, Evans and Laing, 1962). One star, HD 39937, was included on the source list of Weiler and stencel because of its known photometric variability, despite a lack of observed Ca II emission.

The rest of the candidate stars were selected on the basis of their Ca II emission strengths. The intention was to include the strongest southern $\mathrm{Ca}$ II $\mathrm{H}$ and $\mathrm{K}$ emitters with $F, G$ and $K$ spectral types, luminosity classes between III and $V$, and apparent visual magnitudes less than about 8.5 .

In all cases, the presence of $\mathrm{Ca}$ II emission in the candidate stars' spectra had originally been noted by Bidelman and MacConnel1 (1973), Houk and Cowley (1975) or Houk (1978), on University of Michigan objective prism survey plates. 


\section{PHOTOMETRIC SURVEY}

\subsection{INTRODUCTION}

Photometric monitoring was adopted as the initial approach to detecting RS CVn-like surface activity and/or eclipses in the candidate stars.

Priority was given to those stars in the source lists (see Chapter 1) cited as exhibiting particularly strong emission cores in the $\mathrm{Ca}$ II $\mathrm{H}$ and $\mathrm{K}$ lines, and hence presumably high levels of chromospheric heating. The principal aim of the photometric programme was to seek signs of periodic variability in the candidate stars. Wave amplitudes as high as $\sim 0.2$ magnitude have been observed in the northern RS CVn stars II Peg (=HD 224085) and $\lambda$ And (Bopp and Noah, 1980), both of which are single- lined spectroscopic binaries displaying strong $\mathrm{Ca}$ II emission (Rucinski, 1977; Linsky, Worden, McClintock and Robertson 1979). If such wave amplitudes are typical for the strongest Ca II emitters, the likelihood of detecting photometric variability is high, even for stars whose rotation axes lie quite close to the observer's line of sight.

For those stars found to be variable, attempts were made to determine the period of rotation from observations obtained on as many nights as possible. In order to estimate the effective temperatures of the candidate stars, accurate determinations of colour indices were made when conditions permitted. In one star, HD 32918, the wave amplitude was considerably higher in the blue part of the 
spectrum than in the red, and a technique was developed which used this information to assess the area and effective temperature of the spot region.

Over the thirteen months of the survey (1979 August to 1980 September) useful observations were obtained for thirteen stars. In this chapter, the monitoring and data reduction techniques employed are described, and some general results are presented. A more detailed description of the results obtained for individual systems may be found in Chapter 4.

\subsection{EQUIPMENT}

The survey was carried out at Mount John University Observatory, Lake Tekapo, New Zealand. The $61 \mathrm{~cm}$ Boller and Chivens reflector was equipped with a photometer incorporating an RCA C31034A GaAs photomultiplier, cooled to $-30^{\circ} \mathrm{C}$.

The filter combinations used were chosen to approximate the $B, V, R$ and $I$ bands of the cape system described by Cousins (1976).

The tube output electronics permit operation of the system in either analogue (current-to-frequency) or photon counting mode. The latter mode was generally used for stars fainter than $V \sim 7.5$, in order to obtain sufficiently high integration values in ten seconds. A Motorola M6800 microprocessor system controls integration times and records the time of observation, integration value, filter code and object code for each integration, punching them to paper tape for subsequent editing and reduction. 
In order to minimise the effects of extinction, differential measurements were made, interspersing observations of each program star with observations of a nearby comparison star seen through a similar airmass. The comparison stars and check stars were selected so that their colour indices matched those of the corresponding program stars as closely as possible. In no case was the angular separation of program star and comparison greater than $1^{\circ} .5$.

Three integrations of ten seconds each were taken per filter, and the filter sequence $B-V-R-I$ was used for each complete observation of a star. The whole sequence for a single such observation required about three minutes to complete.

No observations were made at zenith angles greater than $60^{\circ}$ (i.e. at more than two airmasses) in order to avoid differential colour extinction effects due to rapidlychanging airmass during the course of a single observation sequence, and to reduce scintillation noise.

Observations of the background sky brightness level in each filter were made every half hour, or more frequently if the sky brightness was changing rapidly (at moonrise, for example). The check star was usually observed once every half hour. A typical observing sequence would thus be

$$
S-C-V-C-K-V-C-V-C-S,
$$

where $\mathrm{S}=\mathrm{sky}, \mathrm{C}=$ comparison star, $\mathrm{V}=$ program star, and $\mathrm{K}=$ check star.

Such a sequence permits accurate subtraction of the sky background and calculation of differential magnitudes 
and colour indices by linear interpolation in time.

Whenever conditions permitted, primary and secondary extinction coefficients were measured nightly employing the method described by Hardie (1962) observing two stars of different colour indices over a wide range of airmasses. Primary extinction was evaluated using the comparison star.

Transformation coefficients and zero point constants for the linear transformations between the instrumental and standard systems were determined from observations of stars in the Harvard E-Regions whose magnitudes and colours in the standard system have been published by Cousins (1973, 1976).

The data reduction was performed on the Burroughs B 6718 system at the University of Canterbury. The main steps in the reduction of a single night's observations of a particular program star were as follows.

\section{(a) Sky subtraction}

An interpolated figure for the background sky integration value was subtracted from the raw integration value for each star, in each filter, after averaging the three integration values taken for each filter.

(b) Conversion to raw magnitudes and colours

Each set of mean integration values minus sky was converted to an arbitrary $\mathrm{V}$ magnitude and three colour indices, using

$$
\begin{aligned}
v & =-2.5 \log \left[I_{v} \times 10^{-4}\right] \\
(b-v) & =-2.5 \log \frac{I_{b}}{I_{v}}
\end{aligned}
$$

and similar expressions for $(v-r)$ and $(v-i)$, where $I_{j}$ is the integration value for filter $j$. 
(c) Extinction correction

Zero-airmass magnitudes and colour indices were evaluated in the instrumental system, using

$$
\begin{aligned}
v_{0} & =v-k_{v}^{\prime} x-k_{v}^{\prime \prime}(b-v) x \\
(b-v)_{0} & =(b-v)\left(1-k_{b v}^{\prime \prime} x\right)-k_{b v}^{\prime} x
\end{aligned}
$$

and similar expressions for $(v-r)_{0}$ and $(v-i)_{0}$, where

$$
\begin{aligned}
& k^{\prime}=\text { primary extinction coefficient } \\
& k^{\prime \prime}=\text { secondary " } \\
& x=\text { airmass at time of observation. }
\end{aligned}
$$

(d) Evaluation of differential magnitudes and colours

The differential zero-airmass values were evaluated

at the time of each observation of the program star and the check star. A linear interpolation in time was used to give the corresponding set of values for the comparison star at the time of observation; these were subtracted in the sense (variable-comparison).

(e) Transformation to standard system

The zero-airmass instrumental differential measures were transformed to the standard system using

$$
\begin{aligned}
& \Delta(B-V)=\mu_{b v} \Delta(b-v)_{o} \\
& \Delta\left(V-R_{C}\right)=\mu_{v r} \Delta(v-r)_{0} \\
& \Delta\left(V-I_{C}\right)=\mu_{v i} \Delta(v-i)_{0} \\
& \Delta V=\Delta v_{o}+\varepsilon \Delta(B-V)
\end{aligned}
$$

where the $\varepsilon, \mu$ are transformation coefficients between the instrumental and cape systems. The subscript "c" denotes the cape system $R$ and $I$ bands, which are somewhat different from the corresponding bands in the Johnson system. (Johnson et al., 1966). The U, B, and V bands, however, are 
essentially the same in both systems.

A program in FORTRAN was written to perform these reductions on the raw data, given the appropriate extinction and transformation coefficients. The program also evaluated heliocentric corrections to be applied to the times of the differential observations, and gave individual magnitudes and colour indices in the standard system for each observation.

\section{$2.4 \quad$ RESULTS}

\subsubsection{Extinction}

Mean values for the extinction coefficients measured at Mt.John during the survey are given in Table 2.1.

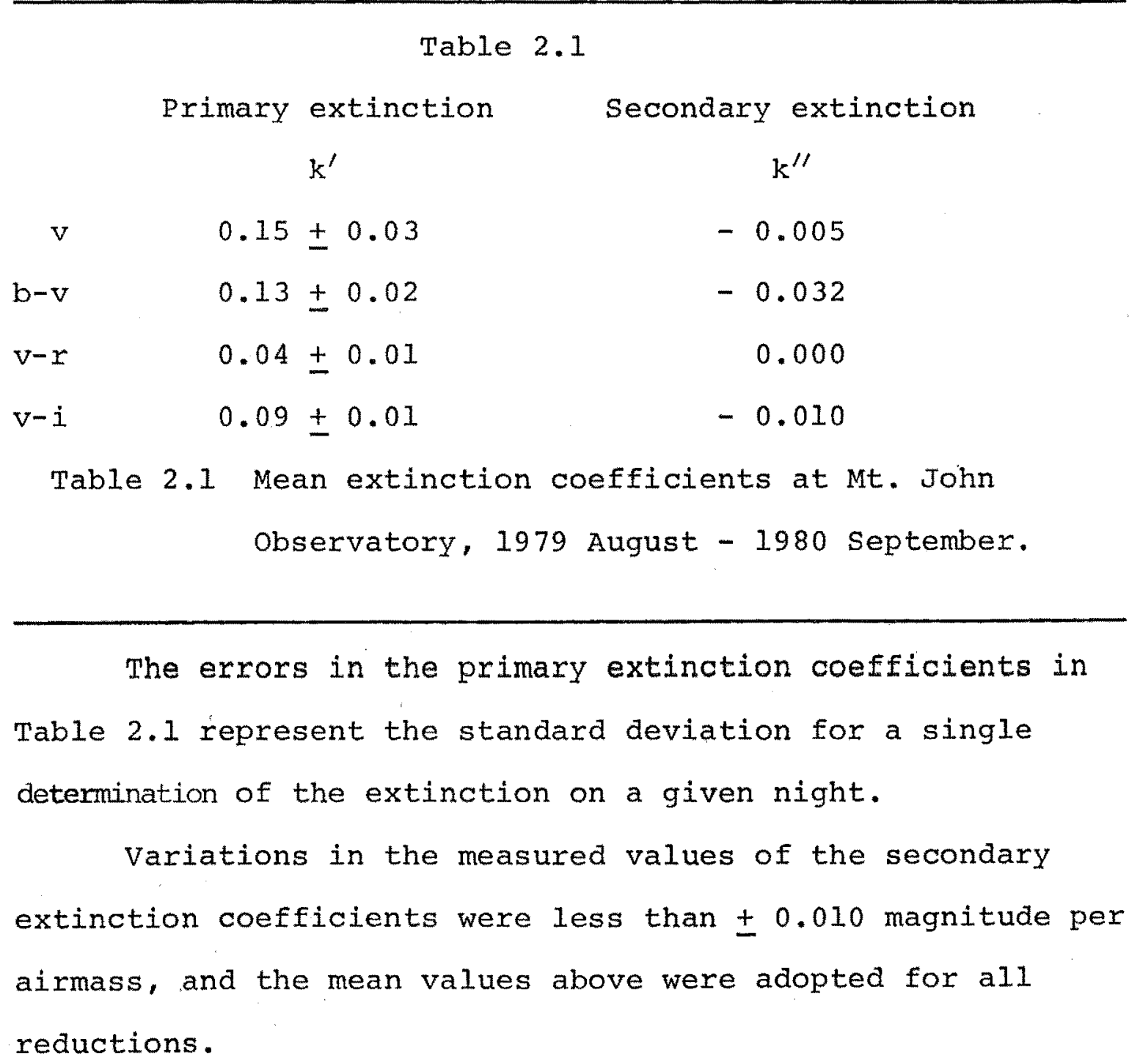


The primary extinction coefficients for the colour indices were apparently independent of the primary $V$ extinction coefficient, $\mathrm{k}_{\mathrm{v}}$, but showed an interdependence described by the mean relations

$$
\begin{aligned}
& \mathrm{k}_{\mathrm{vr}}^{\prime} \simeq 0.23 \mathrm{k}_{\mathrm{bv}}^{\prime} \\
& \mathrm{k}_{\mathrm{vi}}^{\prime} \simeq 0.67 \mathrm{k}_{\mathrm{bv}}^{\prime}
\end{aligned}
$$

Primary extinction values obtained on the same night were used in the reduction of the program star data whenever they were available. Otherwise, the mean values of Table 2.1 were used.

\subsubsection{Instrumental Response}

Night-to-night changes in the overall system sensitivity occasionally amounted to as much as 0.2 magnitude However, examination of data obtained over several hours of continuous observing on individual nights rarely showed drifts in the $\mathrm{V}$ zero point constant of more than 0.01 magnitude during a single night.

The colour sensitivity of the system was found to be extremely stable, and the zero-point constants relating the instrumental colour indices to $(B-V),\left(V-R_{C}\right)$ and $\left(V-I_{C}\right)$ showed no evidence for variations in excess of 0.01 magnitude between 1979 August and 1980 september.

The colour transformation coefficients and zero point constants are listed in Table 2.2. 


\begin{tabular}{|c|c|}
\hline & Table 2.2 \\
\hline & $\begin{array}{l}\text { Zero-point } \\
\text { constant (magnitudes) }\end{array}$ \\
\hline$(b-v)_{0} \rightarrow(B-V)$ & $\zeta_{\mathrm{bv}}=-0.28$ \\
\hline$(v-r)_{0} \rightarrow\left(v-R_{C}\right)$ & $\zeta_{\mathrm{vr}}=-0.13$ \\
\hline$(v-i)_{0} \rightarrow\left(V-I_{c}\right)$ & $\mu_{v i}=1.011$ \\
\hline Table 2.2. & $\begin{array}{l}\text { Transformation coefficients and zero-point } \\
\text { constants relating M.J.U.O. instrumental } \\
\text { colours to Cousins System, } 1979 \text { August - } \\
1980 \text { september. }\end{array}$ \\
\hline Note: & $(A-B)=\mu_{a b}(a-b)_{0}+\zeta_{a b}$ \\
\hline
\end{tabular}

2.4.3 Magnitudes and Colours of Program and Comparison stars

The magnitudes and colour indices of the program and comparison stars in the Cape system are listed in Table 2.3 .

The values given in the table are based on observations made on nights when the primary extinction coefficients and the $\mathrm{V}$ zero-point constant could be determined reliably. In cases where the program star was found to be variable, the $V$ magnitudes listed are based on differential observations made at the time of maximum observed brightness. The amplitude of the observed variation in $\mathrm{V}$ is given in parentheses.

For several stars, UBV photometry was obtained at Mount Stromlo Observatory, A.C.T., Australia during 1981 March-May. The details of this later work are described in Chapter 3; however, for the sake of completeness, the magnitudes and 
colours determined for these stars are included in Table 2.3 .

\section{Table 2.3}

Magnitudes and colour indices in Cousins $U_{B V R} I_{C}$ system for program and comparison stars. Asterisks denote eclipsing binaries.

\begin{tabular}{|c|c|c|c|c|c|c|}
\hline & $\operatorname{tar}$ & $V$ & $(U-B)$ & $(B-V)$ & $(V-R)_{C}$ & $(V-I)_{C}$ \\
\hline $\begin{array}{l}* H D \\
\text { HD }\end{array}$ & $\begin{array}{l}5303 \\
5210\end{array}$ & $\begin{array}{l}7.44(.37) \\
8.72\end{array}$ & - & $\begin{array}{l}0.71 \\
0.58\end{array}$ & $\begin{array}{l}0.44 \\
0.34\end{array}$ & $\begin{array}{l}0.91 \\
0.68\end{array}$ \\
\hline HD & 14643 & $\begin{array}{l}8.19(.15) \\
8.33\end{array}$ & - & $\begin{array}{l}0.82 \\
1.16\end{array}$ & $\begin{array}{l}0.50 \\
0.61\end{array}$ & $\begin{array}{l}0.98 \\
1.17\end{array}$ \\
\hline $\begin{array}{l}\mathrm{HD} \\
\mathrm{HD}\end{array}$ & $\begin{array}{l}32918 \\
33763\end{array}$ & $\begin{array}{l}8.09(.29) \\
8.43\end{array}$ & - & $\begin{array}{l}1.04 \\
0.89\end{array}$ & $\begin{array}{l}0.59 \\
0.49\end{array}$ & $\begin{array}{l}1.19 \\
0.97\end{array}$ \\
\hline $\begin{array}{l}\text { HD } \\
\text { HD }\end{array}$ & $\begin{array}{l}34802 \\
34297\end{array}$ & $\begin{array}{l}7.59(.06) \\
7.37\end{array}$ & - & $\begin{array}{l}1.09 \\
0.66\end{array}$ & $\begin{array}{l}0.59 \\
0.37\end{array}$ & $\begin{array}{l}1.19 \\
0.76\end{array}$ \\
\hline $\begin{array}{l}\mathrm{HD} \\
\mathrm{HD}\end{array}$ & $\begin{array}{l}36705 \\
37379\end{array}$ & $\begin{array}{l}6.38(.04) \\
7.99\end{array}$ & $\overline{-}$ & $\begin{array}{l}0.80 \\
1.45\end{array}$ & $\begin{array}{l}0.46 \\
0.76\end{array}$ & $\begin{array}{l}0.93 \\
1.49\end{array}$ \\
\hline $\begin{array}{l}\mathrm{HD} \\
\mathrm{HD}\end{array}$ & $\begin{array}{l}81410 \\
81904\end{array}$ & $\begin{array}{l}7.13(.06) \\
7.59\end{array}$ & $\begin{array}{l}0.66 \\
0.63\end{array}$ & $\begin{array}{l}1.05 \\
0.98\end{array}$ & $\begin{array}{l}0.58 \\
0.49\end{array}$ & $\begin{array}{l}1.17 \\
0.99\end{array}$ \\
\hline $\begin{array}{l}\mathrm{HD} \\
\mathrm{HD}\end{array}$ & $\begin{array}{l}101309 \\
101679\end{array}$ & $\begin{array}{l}7 \cdot 9:(.11) \\
8 \cdot 0:\end{array}$ & $\begin{array}{l}0.41 \\
0.74\end{array}$ & $\begin{array}{l}0.94 \\
1.11\end{array}$ & - & $\overline{-}$ \\
\hline * $\mathrm{HD}$ & $\begin{array}{l}101379 \\
100382\end{array}$ & $\begin{array}{l}4.8:(.10) \\
5.6:\end{array}$ & $\begin{array}{l}0.26 \\
1.00\end{array}$ & $\begin{array}{l}0.79 \\
1.14\end{array}$ & $\begin{array}{l}0.55 \\
-\end{array}$ & 1.11 \\
\hline $\begin{array}{l}\mathrm{HD} \\
\mathrm{HD}\end{array}$ & $\begin{array}{l}127535 \\
128227\end{array}$ & $\begin{array}{l}8.3:(.36) \\
8.1:\end{array}$ & $\begin{array}{l}0.70 \\
0.73\end{array}$ & $\begin{array}{l}1.07 \\
1.08\end{array}$ & $\overline{-}$ & - \\
\hline $\begin{array}{l}\mathrm{HD} \\
\mathrm{HD}\end{array}$ & $\begin{array}{l}137164 \\
136489\end{array}$ & $\begin{array}{l}7.1:(.22) \\
8.8:\end{array}$ & $\begin{array}{l}0.71 \\
0.68\end{array}$ & $\begin{array}{l}1.04 \\
1.05\end{array}$ & $\overline{-}$ & $\overline{-}$ \\
\hline$\underset{H D}{H D}$ & $\begin{array}{l}155555 \\
156427\end{array}$ & $\begin{array}{l}6.76(.08) \\
7.42\end{array}$ & - & $\begin{array}{l}0.78 \\
1.49\end{array}$ & $\begin{array}{l}0.45 \\
0.80\end{array}$ & $\begin{array}{l}0.87 \\
1.54\end{array}$ \\
\hline $\begin{array}{l}\mathrm{HD} \\
\mathrm{HD}\end{array}$ & $\begin{array}{l}156026 \\
155849\end{array}$ & $\begin{array}{l}6.33(\leqslant .01) \\
7.69\end{array}$ & - & $\begin{array}{l}1.15 \\
1.26\end{array}$ & $\begin{array}{l}0.72 \\
0.73\end{array}$ & $\begin{array}{l}1.30 \\
1.41\end{array}$ \\
\hline $\begin{array}{l}\mathrm{HD} \\
\mathrm{HD}\end{array}$ & $\begin{array}{l}181809 \\
181786\end{array}$ & $\begin{array}{l}6.81(.01) \\
8.70\end{array}$ & - & $\begin{array}{l}1.04 \\
1.09\end{array}$ & $\begin{array}{l}0.60 \\
0.60\end{array}$ & $\begin{array}{l}1.17 \\
1.18\end{array}$ \\
\hline $\begin{array}{l}\text { HD } \\
\text { SAO }\end{array}$ & $\begin{array}{r}185510 \\
0143623\end{array}$ & $\begin{array}{l}8.34(.10) \\
8.28\end{array}$ & - & $\begin{array}{l}1.13 \\
1.11\end{array}$ & $\begin{array}{l}0.67 \\
0.61\end{array}$ & $\begin{array}{l}1.31 \\
1.21\end{array}$ \\
\hline $\begin{array}{l}\mathrm{HD} \\
\mathrm{HD}\end{array}$ & $\begin{array}{l}195040 \\
195106\end{array}$ & $\begin{array}{l}8.96(.13) \\
9.23\end{array}$ & - & $\begin{array}{l}1.13 \\
1.05\end{array}$ & $\begin{array}{l}0.66 \\
0.57\end{array}$ & $\begin{array}{l}1.28 \\
1.08\end{array}$ \\
\hline $\begin{array}{l}\mathrm{HD} \\
\mathrm{HD}\end{array}$ & $\begin{array}{l}196818 \\
196520\end{array}$ & $\begin{array}{l}8.27(.02) \\
7.62\end{array}$ & - & $\begin{array}{l}1.13 \\
1.05\end{array}$ & $\begin{array}{l}0.67 \\
0.58\end{array}$ & $\begin{array}{l}1.33 \\
1.12\end{array}$ \\
\hline
\end{tabular}


Table 2.3 cont...

Notes : 1. Magnitude determinations for the Mt.stromlo UBV data are uncertain by \pm 0.1 magnitude. Al1 other $V$ magnitudes are uncertain by \pm 0.02 magnitude, as are the values for $U-B, B-V, V-R_{C}$ $\mathrm{V}-\mathrm{I}_{\mathrm{C}}$

2. The original comparison star for HD 101379 has been rejected owing to suspected variability; $V-R_{C}$ and $V-I_{C}$ were not determined for the new comparison selected for the UBV programme.

Figures $2.1(a)$ and $(b)$ are plots of $\left(V-R_{C}\right)$ and $\left(V-I_{C}\right)$ respectively, against $(B-V)$. The solid and dashed lines give the mean relations of Johnson (1966) for the main sequence and for unreddened giants, respectively, converted to the Cape system using the relations given by Cousins (1976):

$$
\begin{aligned}
& \left(V-R_{J}\right)=1.40\left(V-R_{C}\right)+0.028 \quad\left(V-R_{C}\right)<1.0 \\
& \left(V-I_{J}\right)=1.30\left(V-I_{C}\right)-0.013 \quad\left(V-I_{c}\right)<2.0
\end{aligned}
$$

The program stars have colour indices typical of objects lying on or near the main sequence. The four bluest program stars have $\left(V-I_{c}\right)$ values rather higher than the mean values for main sequence dwarfs. 

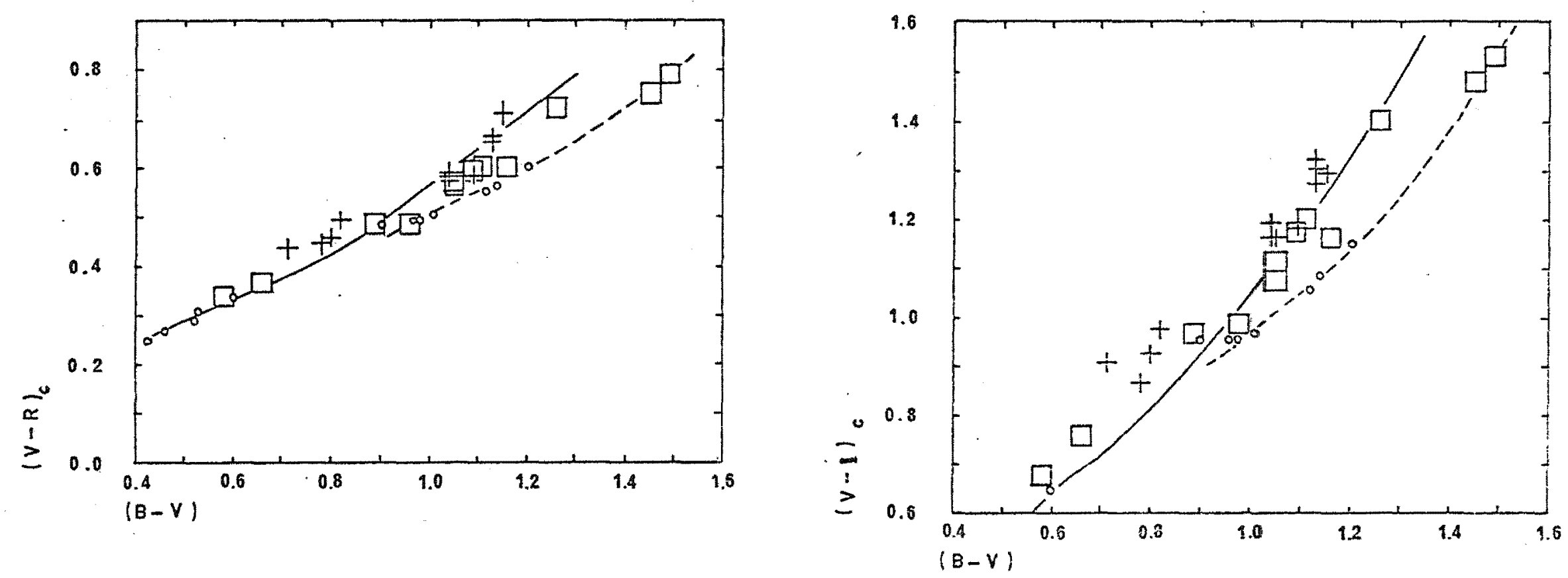

Fig. 2.1

(a) $\left(V-R_{C}\right)$ plotted against $(B-V)$ for program stars (crosses), comparison stars (squares) and several standara stars in the Harvard E-Regions (circles). The solid line represents the mean relation of Johnson (1966) for Iuminosity class $v$, and the dashed line represents the relation for luminosity class III.

(b) $(V-I)_{c}$ plotted against $(B-V)$. Symbols are as for Fig. 2.1 (a). 
Two of these stars, HD 5303 and HD 155555, are known double-lined spectroscopic binaries; the apparent infrared excess is largely an artifact of the superimposed energy distributions of the component stars, which are discussed individually in Chapter 4.

HD 36705 is a peculiar object, a single star with extreme ( $v_{e} \sin i \sim 75 \mathrm{~km} \mathrm{~s}^{-1}$ ) rotational broadening of its spectrum, and an apparent photometric rotation period of 0.514 days (Pakul1, 1981).

HD 14643 is also a fast rotator, whose photometric rotation period seems to be of the order 10 - 20 days, and whose equatorial rotation is $v_{e} \sin i \sim 25 \mathrm{~km} \mathrm{~s} \mathrm{~s}^{-1}$.

In both these cases, the presence of extensive starspot activity, with a large temperature difference between the spot and the photosphere, may be responsible for the apparent infrared excess.

2.4.4 Variability and Rotation Period Analysis

Significant variability was seen in all but one of the program stars, the amplitudes of variation ranging from $0.01 \mathrm{mag}$ (HD 181809) to $0.32 \mathrm{mag}$ (HD 32918). Both HD 5303 and HD 101379 (= HR 4492) were discovered to be eciipsing binaries.

In most cases, insufficient data were available for a period analysis of the photometric variations to give any meaningful results. In the cases of $\operatorname{HD} 32918,34802$, 185510 and 195040, however, preliminary period analyses yielded possible rotation periods.

The method chosen for the analysis was similar to that described by Gieseking (1981) for period analysis of radial velocity observations. The method involves fitting a sinusoid to the observations by least squares, for each 
member of a set of trial periods. The r.m.s. deviation of the observations from the fitted curve is evaluated for each trial period, and a plot of these residuals against $\log \mathrm{P}$ is constructed. Minima in the residuals indicate likely periods for the variation.

This technique is well-suited to period analyses of radial velocity data for low-eccentricity spectroscopic binaries, and its use for this purpose will be described again in chapter 3. In most cases, the photometric variation resulting from RS CVn-like surface activity is a rather poor approximation to a sinusoid. Nonetheless, if sufficient data are available, the period of the lowest harmonic present in the data is usually fairly readily determined from a plot of the residuals against $\log \mathrm{P}$ (fig. 2.2).

The complicated aliasing structure seen in the periodograms arises from undersampling of the data. Observing runs were generally scheduled during bright-sky time, and (depending on weather conditions) useful data were typically obtained on only three to four nights per month.

The spacing of the minima in the aliasing pattern is dependent on the intervals between observing runs. Families of minima occur at periods $\mathrm{P}_{\Delta t, \mathrm{n}}$ given by

$$
\mathrm{P}_{\Delta t, n} \frac{\mathrm{I}}{\mathrm{P}_{0}} \pm \frac{\mathrm{I}}{\Delta \mathrm{t}} \mathrm{n}=0,1,2, \ldots
$$

where $P_{0}$ is the true period in days and $\Delta t$ is the interval in days between any two sets of observations. Several such families, with $\Delta t$ being multiples of one month, are seen in most cases. Careful analysis of the aliasing pattern generally revealed the most likely period. The preliminary periods obtained for these four stars are given in Table 2.4 . 
Fig. 2.2 Periodograms for four program stars, generated using the method of Gieseking (see text). In each case, "Po" denotes the most' likely rotation period, as determined from the aliasing pattern 


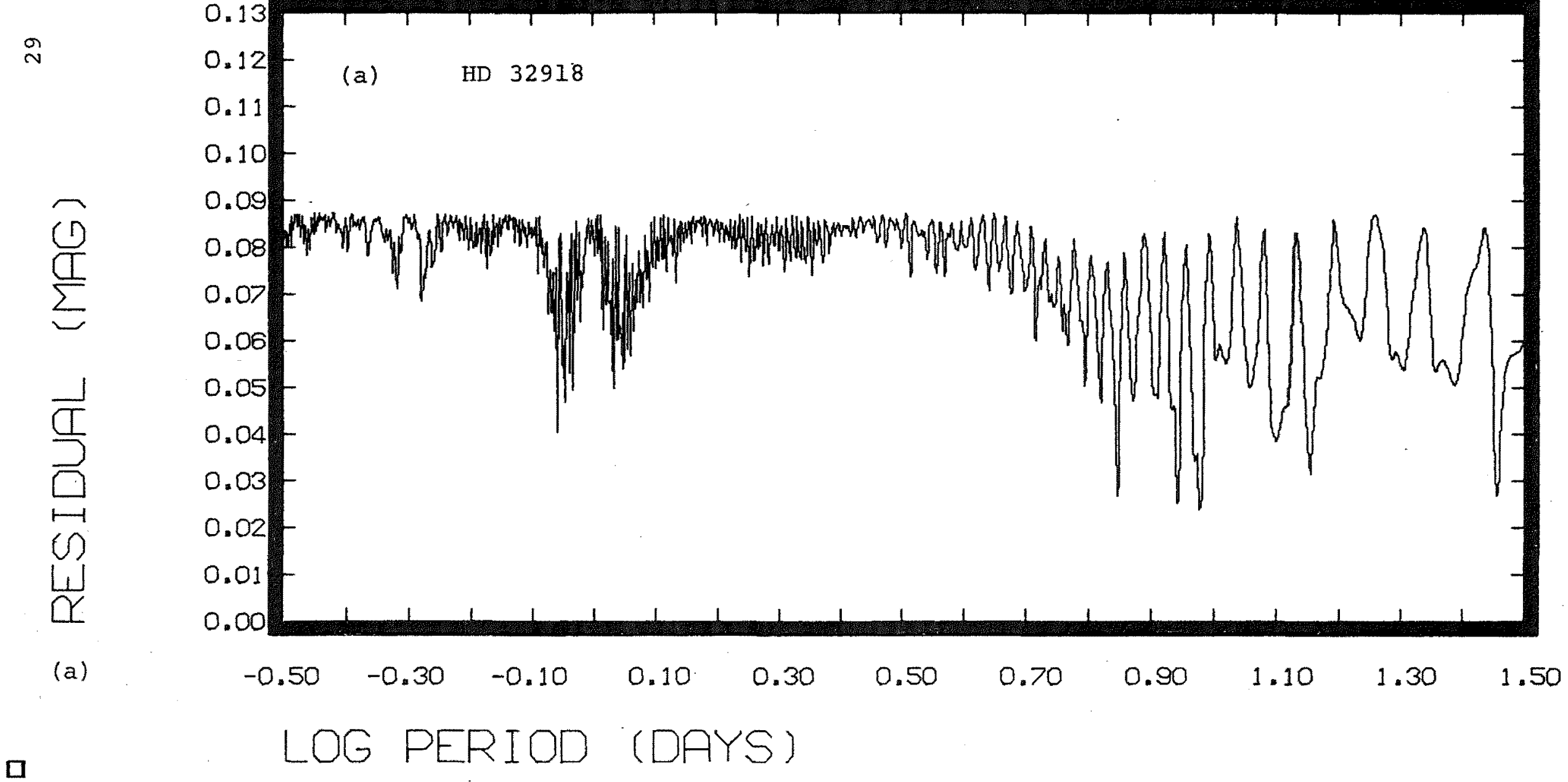




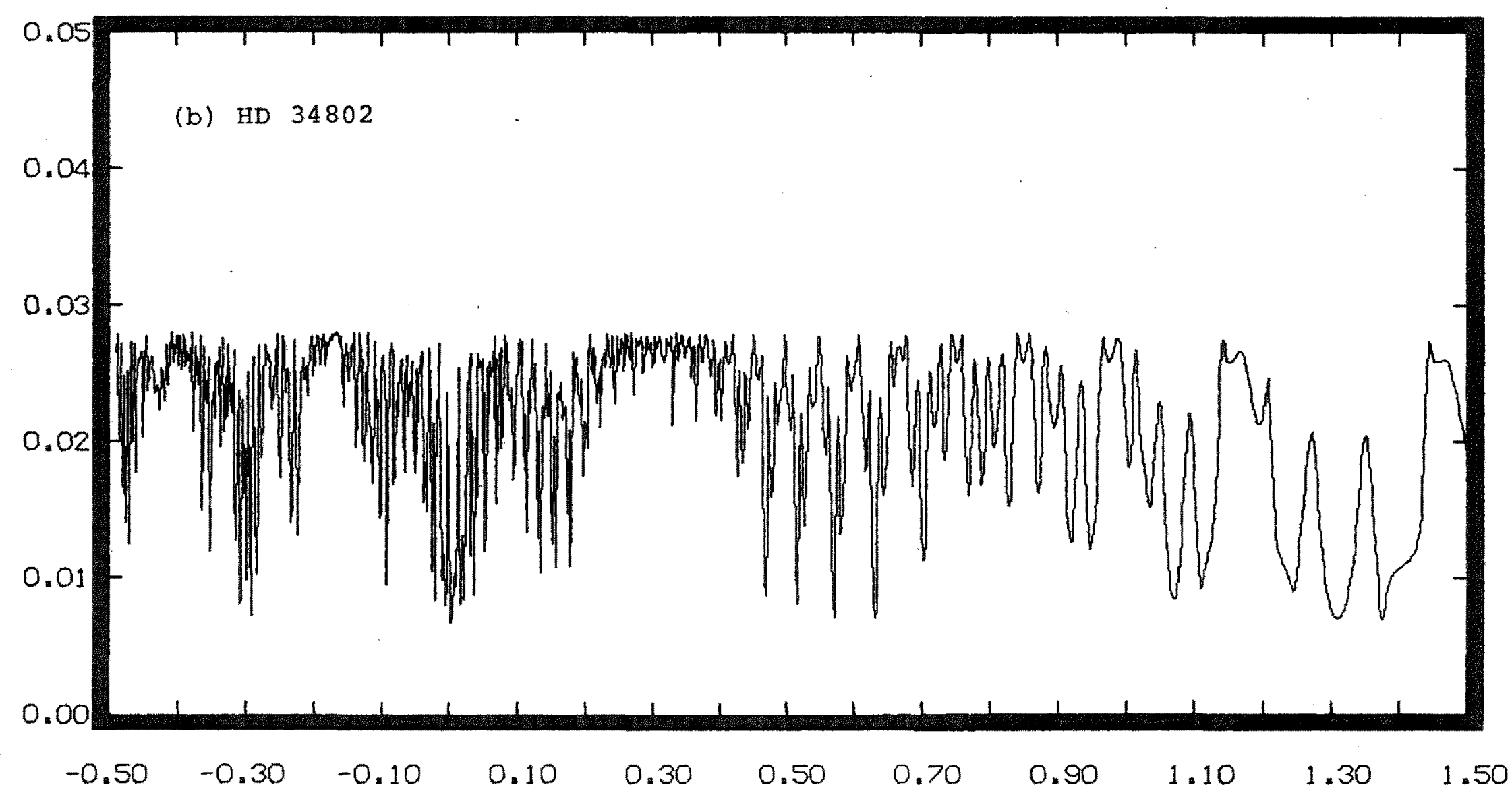

$\square$

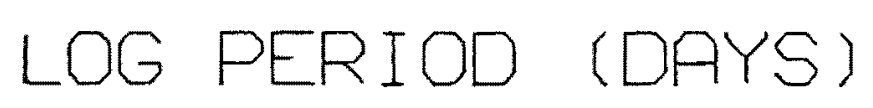




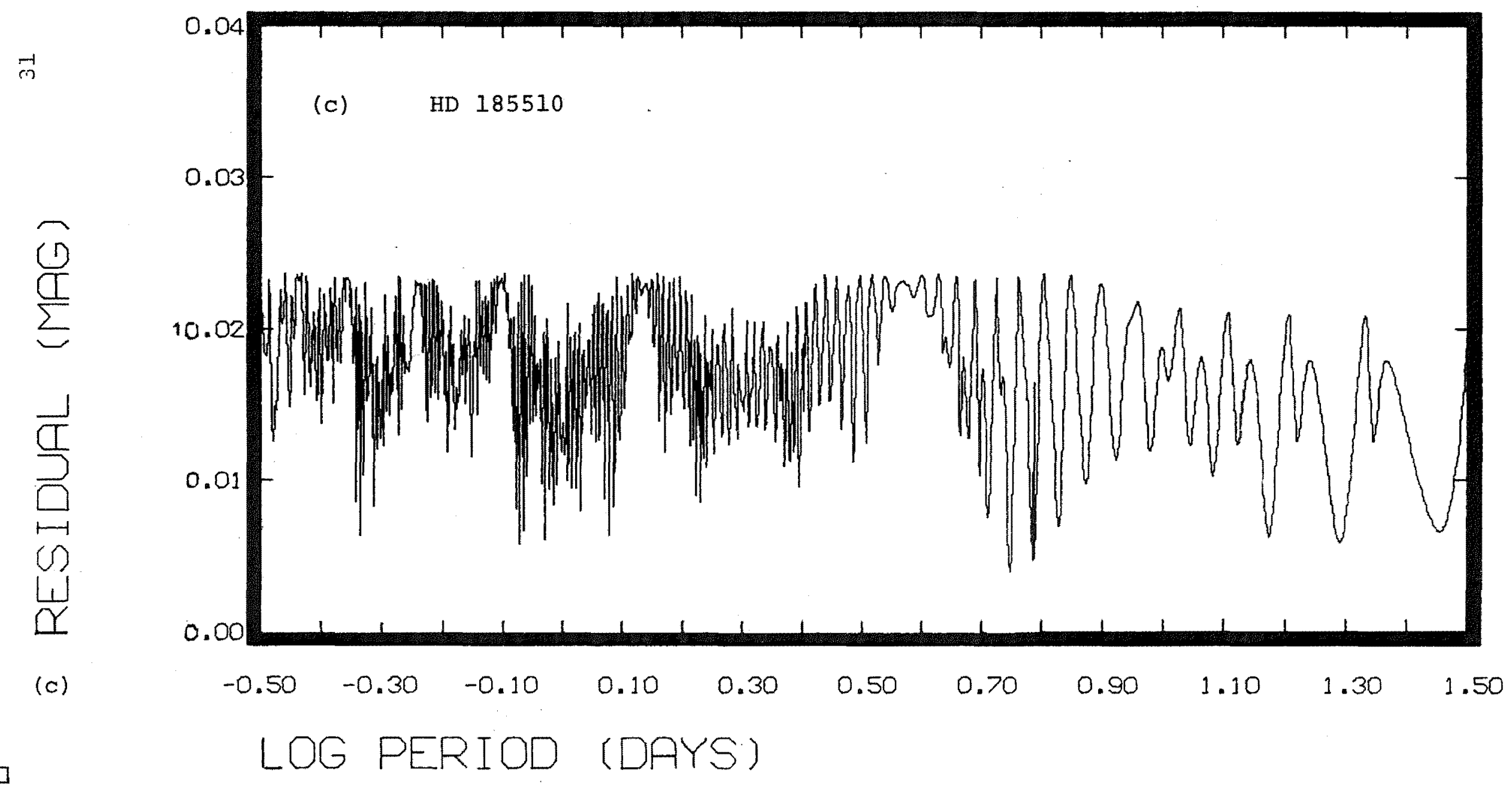




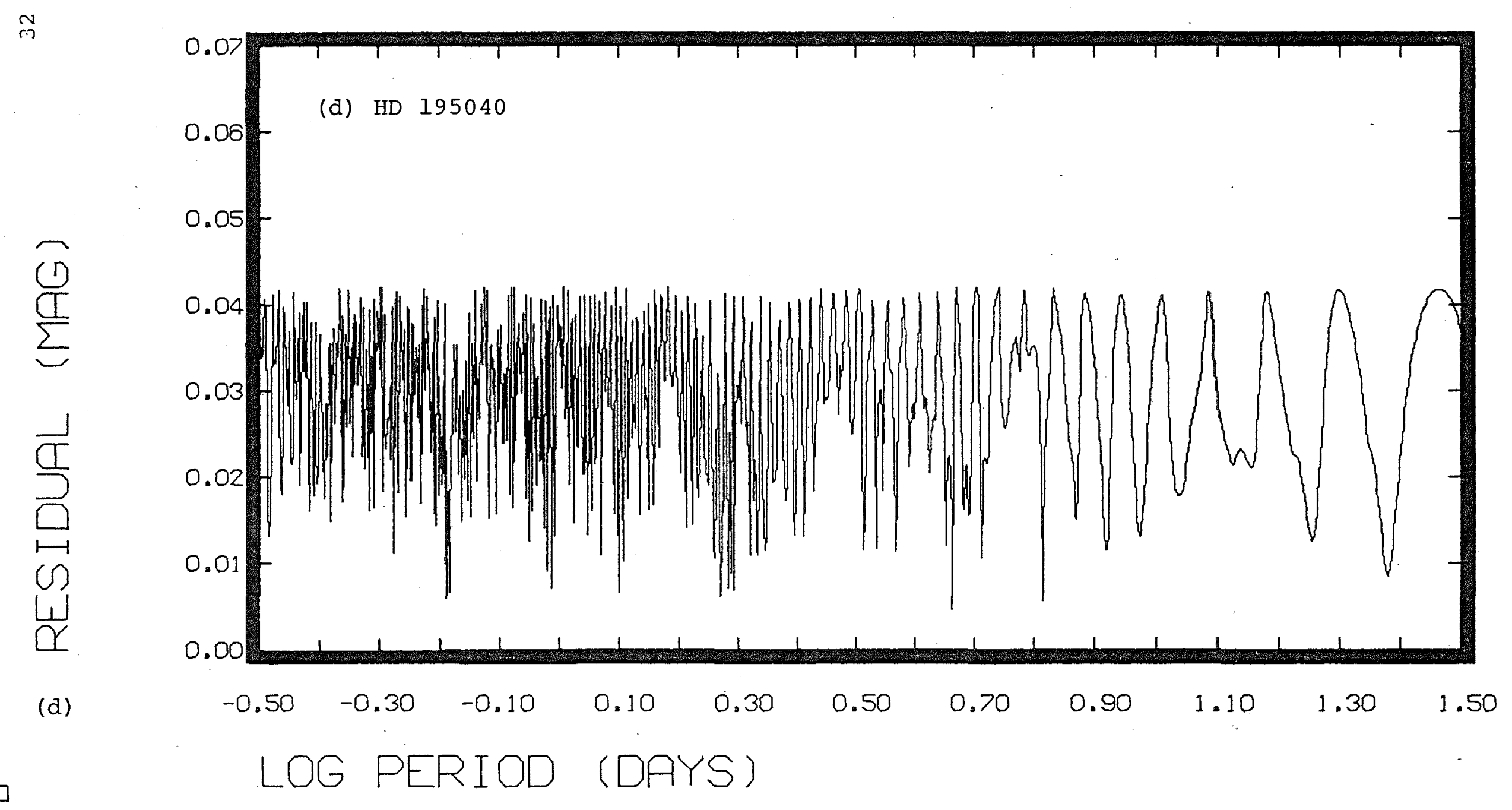


Table 2.4

$\begin{array}{ccc}\begin{array}{c}\text { Star } \\ \text { HD }\end{array} & \begin{array}{c}\text { Most likely } \\ \text { Rotation period }\end{array} & \text { (days) } \\ 32918 & 9.55 \pm 0.02 & \text { A } \\ 34802 & 20.36 \pm 0.06 & \text { B } \\ 185510 & 6.12 \pm 0.02 & \text { B } \\ 195040 & 8.27 \pm 0.02 & \mathrm{C}\end{array}$

Table 2.3 Photometrically determined rotation periods for four stars. The reliability of each period is a subjective estimate based on the ease of identifying $P_{0}$ from the aliasing pattern.

In the case of HD 195040, the period of 8.27 days was selected because it coincided with one of the likely orbital periods determined from the star's radial velocity curve. The system is discussed in more detail in Chapter 4.

\subsubsection{Starspot size and Temperature}

In surface-active stars exhibiting large-amplitude rotational variability, the highly uneven longitudinal surface brightness distribution may give rise to variations in the colour indices with varying spot aspect, provided that the surface brightness of the spot (at least in the red part of the spectrum) is not negligibly small compared with that of the photosphere.

The magnitude depression due to the spot in a band with an effective wavelength $\lambda$ may be expressed in the form 


$$
\Delta \mathrm{m}_{\lambda}=-2.5 \log \left[1-\alpha_{\lambda}\left(1-\beta_{\lambda}\right)\right]
$$

where $\alpha_{\lambda}$ is the fractional area of the star's visible hemisphere (seen projected on the sky) occupied by the spots, and $\beta_{\lambda}$ is the ratio of the specific intensity $s_{\lambda}^{\prime}$ of the spot (at the disc centre) to that of the surrounding photosphere, $\mathrm{s}_{\lambda}$.

Vogt (1981) decouples the effects of limb darkening and apparent spot area from those of the temperature difference between the spot and the photosphere, by defining

$$
\alpha_{\lambda}=\iint_{\frac{\left(1-u_{\lambda}+u_{\lambda} \cos \gamma\right) \cos \gamma \sin \theta d \theta d \phi}{\pi\left(1-\frac{u_{\lambda}}{3}\right)}}
$$

where $u_{\lambda}$ is the limb darkening coefficient,

$\gamma$ is the angle of foreshortening,

$\theta$ is the stellar co-latitude, and

$\phi$ is the stellar longitude.

$\alpha_{\lambda}$ thus represents an effective spot "area" which incorporates the effect of limb darkening on the apparent area of spots located near the limb of the star's disc. It is assumed that the limb darkening coefficient $u$ has the same value for both the spot and the photosphere.

Equation (2.6) may be re-arranged to give

$$
\alpha_{\lambda}=\frac{\operatorname{dex}\left(-\Delta \mathrm{m}_{\lambda} / 2.5\right)-1}{\beta_{\lambda}-1}
$$

The variation of $\alpha_{\lambda}$ with wavelength is determined by the variation of the limb darkening coefficient is with wavelength. In the $V, R$ and I bands, the limb darkening coefficient for a late $G$ for early $\mathrm{K}$ dwarf or subgiant has a value of about 0.6 (Grygar, Cooper and Jurkevich, 197.2), 
showing only a weak wavelength dependence. In this case,

$$
\begin{aligned}
& \alpha_{V}=\alpha_{R} \text { and we write } \\
& \frac{\beta_{R}-1}{\beta_{V}-1}=\frac{\operatorname{dex}\left(-\Delta m_{R} / 2.5\right)-1}{\operatorname{dex}\left(-\Delta m_{V} / 2.5\right)-1}
\end{aligned}
$$

Vogt (1981) then uses the Barnes-Evans relationship between Johnson $V-R$ colour index and the visual surface brightness parameter $F_{V}$ to obtain the difference between the $V-R$ colour indices of the spot and the photosphere:

$$
\begin{aligned}
\mathrm{F}_{\mathrm{V}}=4.2207- & 0.1 \mathrm{~V}_{\circ}-0.5 \log \phi^{\prime} \\
\text { where } \mathrm{V}_{\mathrm{O}} & \text { is the unreddened } \mathrm{V} \\
& \text { magnitude, and } \phi \text { is } \\
& \text { the angular diameter } \\
& \text { of the star (Barnes, } \\
& \text { Evans and Moffett,1978). }
\end{aligned}
$$

Now

$$
\mathrm{V}_{\mathrm{O}}=-2.5 \log \mathrm{S}_{\mathrm{v}}+\text { const. }
$$

Hence

$$
\log S_{V}=4 F_{V}+\text { const. }
$$

and

$$
\beta_{v}=\frac{S_{v}^{\prime}}{S_{v}}=\operatorname{dex}\left[4\left(F_{v}^{\prime}-F_{v}\right)\right]
$$

Similarly

$$
\beta_{R}=\frac{S_{R}^{\prime}}{S_{R}}=\operatorname{dex}\left[4\left(F_{v}^{\prime}-F_{V}\right)\right]-0.4\left((V-R)_{\text {star }}-(V-R)_{\text {spot }}\right)
$$

Now

$$
\begin{gathered}
F_{V}=3.841-A(V-R) \circ \text { where } A=0.321 \pm 0.001 \\
\text { for stars with }(V-R)_{0} \geqslant 0.7 . \\
\quad \text { (Barnes, Evans and } \\
\text { (Moffett, 1978). }
\end{gathered}
$$


Hence

$$
\begin{aligned}
F_{V}^{\prime}-F_{V}= & A D \\
& \text { where } D=(V-R)_{\text {star }}-(V-R)_{\text {spot }}
\end{aligned}
$$

By combining Eqs. 2.11, 2.12 and 2.14 and substituting for $\beta_{R}$ and $\beta_{V}$ in $E q \cdot 2.7$, Vogt obtains

$$
=\frac{\operatorname{dex}[4 D(A-0.1)]-1}{\operatorname{dex}[4 A D]-1}=\frac{\operatorname{dex}\left(-\Delta m_{R} / 2.5\right)-1}{\operatorname{dex}\left(-\Delta m_{v} / 2.5\right)-1}
$$

This may be solved iteratively, to obtain the difference $D$ between the Johnson $(V-R)$ colour indices of the spot and the photosphere. The mean colour index/temperature relations of Johnson (1966) are then used to obtain the effective temperatures of the spot and the photosphere, using the star's $(V-R)$ colour index at wave maximum.

The effective fractional spot area at wave minimum is then given by

$$
\alpha=\frac{\operatorname{dex}\left(-m_{v} / 2.5\right)-1}{\operatorname{dex}(4 A D)-1}
$$

Although this method is capable of yielding the spot temperature to within $\pm 200 \mathrm{~K}$ or so, it will only yield an accurate difference between the photospheric and spot temperatures (and hence an accurate spot area) if the hemisphere visible at wave maximum is free from starspots. This can only be assumed if the lightcurve shows clearly "flat-topped" maxima. For stars which exhibit continuous variation in magnitude and colour near wave maximum, the photospheric temperature will generally be under-estimated, because of the contribution of the spotted area still visible at wave maximum. 
of the stars for which extensive lightcurve coverage was obtained, only HD 32918 and HD 5303 were found to exhibit spot waves of such an amplitude that the colour changes were readily observable.

The calculation of the spot temperature in HD 5303 is complicated by the presence of the light from the hotter secondary component in the binary, and is discussed in the paper by Collier, Hearnshaw and Austin (1981).

HD 32918 is a member of the FK Comae class of single, rapidly-rotating convective giants (Bopp and Stencel, 1981). The calculation of the size and temperature of the spot region in this star is described fully in Chapter 4 . 


\section{HIGH RESOLUTION SPECTROSCOPY}

\subsection{INTRODUCTION}

The high incidence of variability found among the stars observed in the course of the photometric survey at Mt.John suggested that RS CVn-like starspot activity accompanied the observed strong $\mathrm{Ca}$ II $\mathrm{H}$ and $\mathrm{K}$ emission in nearly all cases.

However, little further information concerning the dimensions of the observed systems could be obtained through continued photometry. The low-resolution spectroscopic survey by Hearnshaw (1978) consisted of a large number of blue plates taken at Mt.John, at a dispersion of $60 \AA \mathrm{mm}^{-1}$ giving velocity measures with a standard error of $10 \mathrm{~km} \mathrm{~s}^{-1}$. Several spectra each of the strong Ca II emitters HD 5303, 34802, 36705, 101309, $101379,137164,155555,156026$ and 196818 were included, but measurable radial velocity variations were only present for the double-lined binaries HD 5303 (Hearnshaw and oliver, 1977; Collier, Hearnshaw and Austin, 1981) and HD 155555 (Hearnshaw, 1979). Some luminosity information could be gleaned from visual inspection of the line ratio $\lambda 4077 \mathrm{SrII} /$ $\lambda 4071 \mathrm{FeI}$ on the place tracings, and indicated that the luminosity classes of the stars surveyed ranged from III to V.

Four high-resolution spectroscopic surveys were carried out at the Mount stromlo (MSO) and siding spring (SSO) Observatories of the Australian National University, during late 1980 and early 1981.

The first survey, carried out during 1980 october and November, sought evidence of radial velocity variations 
in spectra of the $\mathrm{Mg}$ I b triplet region around $5170 \AA$.

The second survey consisted of three observing runs in 1980 December, 1981 February and $1981 \mathrm{July}$. High resolution spectra of the $\mathrm{H} \alpha$ profiles of the candidate stars were obtained and were examined for signs of flare activity in the form of emission or unusually shallow $\mathrm{Ha}$ absorption profiles. Secondary aims of the survey were

(a) to assess the degree of $\mathrm{H} \alpha$ variability and, if possible, to look for signs of rotational modulation of the emission strength;

(b) to measure the degree of rotational smearing of the weak metallic absorption lines near $\mathrm{H} \alpha$, and hence obtain values of $v_{e} \sin i$; and

(c) to evaluate the radial velocity of the metallic line spectrum.

The aim of the third and fourth surveys was a detailed analysis of the $\mathrm{Ca}$ II $\mathrm{H}$ and $\mathrm{K}$ emission lines in nine stars. Coudé spectra covering a $120 \AA$ band centred on $3950 \AA$, with. a resolution of $0.3 \AA$ were obtained during 1981 March using the MSO $1.88 \mathrm{~m}$ telescope and were analysed using the calibration method developed by Linsky, Worden, Mcclintock and Robertson (1979) to obtain absolute surface fluxes (and hence net chromospheric cooling rates) for the program stars.

A more extensive survey of eight stars was carried out during 1981 March and April, using the Cassegrain échelle spectrograph on the sso $1.0 \mathrm{~m}$ telescope. These spectra covered a $40 \AA$ band centred on the $K$ line, with a higher dispersion but similar resolution to the MSO spectra. The aims of this survey were

(a) to obtain accurate surface gravities for the program stars, from the Wilson-Bappu emission core 
(b) to seek evidence of rotational modulation of the Ca II K emission flux.

A simultaneous program of UBV photometry was carried out between 1981 March and May, in order to establish epochs of distortion wave minima in the program stars, for comparison with any regular variation found in the Ca II $K$ emission flux.

Radial velocities were evaluated for both the emission and absorption features in the Ca II spectra. These were combined with the radial velocities obtained from the $\mathrm{Mg} \mathrm{I} \mathrm{b}$ and $H \alpha$ spectra, and the period analysis technique of Gieseking (1981) was applied to those stars for which sufficient data was available for a preliminary radial velocity solution to be obtained.

\subsection{INSTRUMENTATION AND OBSERVATIONAL TECHNIQUES}

\subsubsection{Mg I b coudé spectra}

For the purpose of detailed radial velocity analysis of as many stars as possible, the region of the $\mathrm{Mg} I \mathrm{~b}$ triplet near $5170 \AA$ was chosen as being particularly suitable for late-type stars with (in some cases) rotationally-smeared spectra. The absorption lines in this spectral region are well-defined even in the presence of rotational smearing amounting to $\mathrm{v}_{\mathrm{e}} \sin i \sim 70 \mathrm{~km} \mathrm{~s}^{-1}$. The combination of the stellar energy distribution and the $s-20$ response of the detector permitted high signal-to-noise ratios to be obtained with short exposure times at this wavelength.

The spectra were obtained using the "C" grating 1600 lines $\mathrm{mm}^{-1}$, blazed at $12000 \AA$ in the first order) at the 
f/31.7 coudé focus of the $1.88 \mathrm{~m}$ telescope at MSO. The grating was used in its second order. The focal lengths of the collimator and camera were 5.64 , and $0.813 \mathrm{~m}$ respectively.

The detector used was the one-dimensional photon counting array (PCA) developed at MSO (Stapinski, Rodgers and Ellis, 1979). The device consists of a six-stage image intensifier with $\mathrm{s}-20$ photocathode response, focussed onto dual Reticon arrays consisting of 512 elements each, on $25 \mu$ centres in the dispersion direction. Each photon pulse can be located to within half an element in the dispersion direction, giving an effective array length of 1024 elements on $12.5 \mu$ centres. During an exposure the data are transferred to a fast-incrementing memory. Exposure times and data displays are controlled by a PDP/11 computer, which may access the PCA memory at any time during exposure. Upon completion of an exposure, the data are written to magnetic tape, with a file header incorporating information on the start time and duration of the exposure and telescope coordinates.

In typical seeing conditions, the star images were between 3 and 5 arc seconds in diameter. A typical slit width of $350 \mu$ (i.e. a projected slit width of 1.0 arc second) gave a resolution

$$
\mathrm{R}=\frac{\lambda}{\Delta \lambda}=1.3 \times 10^{4},
$$

or $\Delta \lambda \simeq 0.4 \AA$ at $5170 \AA$. The effective dispersion at the Reticon was $0.174 \AA \mathrm{pixel}^{-1}$ or $13.9 \mathrm{Am}^{-1}$. The discrepancy between this figure and the calculated $9.6 \AA \mathrm{mm}^{-1}$. for the spectrograph configuration arises in the optical system used to focus the phosphor image from the intensifier on to the Reticon. 
With this configuration it was found that in typical seeing conditions and using a slit width of $350 \mu$, a $G-K$ star with a $V$ magnitude of $\sim 8.5$ gave a mean count rate per pixel of about $2 \mathrm{~Hz}$. For brighter stars, neutral density filters were used to give count rates between 2 and $5 \mathrm{~Hz}$, and exposure times were adjusted so as to give a total mean count rate close to 900 photon events per channel, with a corresponding signal to statistical photon noise ratio of about $30: 1$.

Exposures of a neon-iron arc spectrum were made before and after each stellar spectrum, for wavelength calibration purposes. A "flat-field" exposure of an incandescent continuum source was made at the end of each night, to aid the removal of fixed-pattern detector noise from the spectra during data processing. In order to minimise photon noise, the flat-field exposure was made at a count rate near $3 \mathrm{~Hz} /$ pixel for about 1000 seconds, giving a signal-to-noise ratio two or three times greater than that of an average stellar spectrum.

\subsubsection{Ha coudé échelle spectra}

The $\mathrm{H} \alpha$ spectra were obtained using the same collimator, camera and detector arrangement on the MSO $1.88 \mathrm{~m}$ telescope already described for the Mg I b coudé spectra. An échelle grating (79 lines $\mathrm{mm}^{-1}$ with blaze angle $63^{\circ} 26^{\prime}$ ) was used in its $35^{\text {th }}$ order, in conjunction with the " $E$ " grating (300 lines $\mathrm{mm}^{-1}$, blazed at $5000 \AA$ in the first order) as cross-disperser.

The effective dispersion of this configuation was 2.7 $\AA \mathrm{mm}^{-1}$ at the Reticon array, or $0.034 \AA$ per $12.5 \mu$ pixel. A slit width of $200 \mu$ gave a resolution $\Delta \lambda \simeq 0.2 \AA$ at $\lambda=6560 \AA$. and in typical seeing a $V=8.5$ star with spectral type near 
KO gave a mean count rate per pixel of about $0.1 \mathrm{~Hz}$. Exposure times of up to 3600 s were used; signal-to-noise ratios of about 14 were obtainable for the faintest stars observed $(V \sim 9.0)$ in this time.

Flat-field spectra of an incandescent source were taken on each night, using the same technique described for the Mg I b spectra.

3.2.3 Ca II $\mathrm{H}$ and $\mathrm{K}$ coudé spectra

The Ca II $H$ and $K$ spectra were obtained at the coudé focus of the MSO $1.88 \mathrm{~m}$ telescope, using the same collimator, grating, camera and detector arrangement already described for the Mg I b spectra.

The grating was used in its third order. In order to cut out the red wavelengths from the second order, a liquid $\mathrm{CuSO}_{4}$ filter and a $2 \mathrm{~mm}$ schott BG 25 filter were used.

The effective dispersion at the Reticon array was $0.112 \AA$ per $12.5 \mu$ pixel; a typical slit width of 1 arc second gave a resolution of $0.31 \AA$ at $3950 \AA$. The spectra covered the wavelength rage 3890-4010 $\AA$. Neon-iron arc spectra were taken with each stellar spectrum, for wavelength calibration purposes.

\subsubsection{Ca II $K$ Cassegrain échelle spectra}

The échelle spectra of the Ca II K line emission cores of the program stars were obtained with the Boller and Chivens échelle spectrograph at the $f / 10$ Cassegrain focus of the $1.0 \mathrm{~m}$ telescope at siding spring Observatory. The spectrograph comprises a collimator and camera with focal lengths $1.0 \mathrm{~m}$ and $0.303 \mathrm{~m}$ respectively; a 79 line $\mathrm{mm}^{-1}$ échelle grating was used, with a 6001 ine $\mathrm{mm}^{-1}$ grating 
(blazed at $7500 \AA$ in the first order) in its second order as the cross-disperser.

The detector was the two-dimensional photon counting array (2D PCA) developed at Mount Stromlo observatory (Stapinski, Rodgers and Ellis, 1981). The system consists of a dual microchannel plate intensifier followed by a singlestage electrostatic-focus tube. The phosphor image is focussed onto a Fairchild CCD type SL 62925 , consisting of a $488 \times 380$ array of $12 \times 18$ micron charge-coupled photosites. Each photon pulse spreads over about four elements in each direction, but can be located with an accuracy of about half an element. In the present system, photon events are located to within half a diode in the dispersion direction and to one diode in the other direction, giving an effective array size of $760 \times 488$ pixels.

The photon pulses are transferred to a fast-incrementing memory with a capacity of $512 \mathrm{~K}$ 16-bit words. Exposure times and data displays are controlled by a PDP/11 computer; at the completion of each exposure the data are recorded on magnetic tape.

In typical seeing conditions the image diameters of the stars were about 4-6 arc seconds. In order to obtain sufficiently high count rates per pixel for the fainter program stars to give reasonable signal-to-noise ratios, projected slit widths of up to 4 arc seconds were necessary. The corresponding slit width setting was $200 \mu$, and gave a resolution $\Delta \lambda=0.28 \AA$ at $\lambda \sim 3935 \AA$. The effective dispersion at the CCD was $0.053 \AA$ per pixel at this wavelength The vertical extent of each order of the échelle spectrum was between 3 and 5 rows of the CCD array, depending on the seeing conditions. The sum of the count rates over the 
rows occupied by the spectrum was about $0.03 \mathrm{~Hz}$ per channel in the dispersion direction for $a \mathrm{~V} \simeq 8.5$ star of spectral type near ko. A signal-to-noise ratio of about 10 was thus attainable in a 3600-second exposure for such a star.

The spectra covered the wavelength range 3915 to

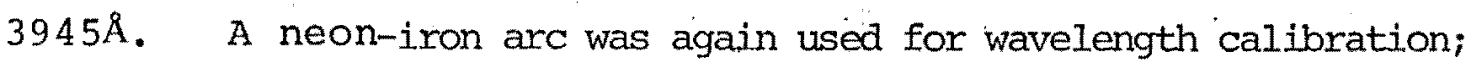
arc spectra were taken before and after each stellar spectrum. Subsequent examination of the wavelength calibration data showed that the system was very stable, with negligible drifts in the wavelength calibration occurring during the course of a night with changing telescope attitude.

\subsubsection{UBV Photometry}

A program of broad-band differential photometry in the UBV standard system was carried out over a total of 17 nights during 1981 March to May.

The single-channel photon-counting photometer on the $0.76 \mathrm{~m}$ Boller and Chivens telescope at MSO was employed for this purpose. A dry ice-cooled IP2I photomultiplier was used in conjunction with a general-purpose integrating system controlled by a PDP/11 computer.

A similar observing routine to that described for the MJUO photometric survey (Chapter 2) was adopted. Sets of three ten-second integrations per filter were used; observation times, telescope coordinates, integration values and filter codes were stored on disk for later reduction on the same computer. 
All spectra obtained at Mount stromlo and Siding Spring Observatories were analysed using the SPECT interactive spectral reduction package on the VAX $11 / 780$ computer at Mt. Stromlo.

\subsubsection{Preliminary Treatment}

The first step in the reduction of the raw spectra involved the removal of the fixed noise pattern inherent in the detector. The pattern took the form of a "ripple" with a cycle length of four pixels, generated by clock noise from the detector electronics. The amplitude of the ripple was about $3 \%$ of the mean spectrum level.

The flat-field spectra taken on each night were normalised to give a mean value per pixel of 1.0. Each stellar spectrum was then divided, pixel by pixel, by the normalised flat-field spectrum, effectively eliminating the fixed noise pattern.

\subsubsection{Wavelength Calibration}

Each stellar spectrum was next calibrated from measurements of the positions in the array (in pixel units) of lines of accurately known wavelength in the accompanying neon-iron arc spectra. Each arc line was spread over about four pixels, and the line centres could be determined with an accuracy of about 0.2 pixel. A third order polynomial fit was found to represent adequately the variation of wavelength with pixel number. The spectra were calibrated individually, and it was found that the r.m.s. deviation of the known arc line wavelengths from the values obtained using the polynomial fit was typically about $0.1 \AA$. 
3.3 .3 Radial Velocity Reductions

The cross-correlation method of simkin (1974) was used to evaluate the heliocentric radial velocity of each stellar spectrum.

The method involves correlating the spectrum whose radial velocity is to be calculated, with a "template" spectrum of a star of similar spectral type and accurately determined radial velocity, in the Fourier domain.

Preparation of the data for this process involves re-binning each spectrum into an array with $\mathrm{N}$ pixels, so that the relationship between pixel number $\mathrm{x}$ and wavelength $\lambda$ takes the form

$$
\begin{aligned}
\mathrm{x}=\mathrm{A} & \log \lambda+\mathrm{B} \\
& \text { where } \mathrm{A}+\mathrm{B} \text { are constant. }
\end{aligned}
$$

This arrangement is such that a difference in radial velocity $\Delta v$ between two spectra will be seen as a uniform relative shift in pixel units along the entire length of the spectrum

$\Delta x($ pixel units $)=A \Delta(\log \lambda)=\frac{1}{2} A \log \left[\frac{1-\frac{\Delta v}{c}}{1+\frac{\Delta v}{C}}\right]$

The cross-correlation function (c.c.f) of a stellar spectrum $G(x)$ with a template spectrum $T(x)$ is defined as

$$
\begin{aligned}
C(z) \equiv a \int_{-\infty}^{\infty} T(x) G(x+z) d x \\
\text { where } a^{-1} \text { is a scaling factor }
\end{aligned}
$$

equal to the product of the mean square values of. $T$ and $G$, when the means of $T$ and $G$ have been set to zero.

$C(z)$ will have a maximum at the lag $z=z_{0}$, which represents the shift (in pixel units) of the spectrum under examination with respect to the template spectrum. This yields directly the difference between their geocentric 
radial velocities.

In the Fourier domain, the transform of the c.c.f. is

$$
\begin{aligned}
c(f)= & g(f) t(-f) \\
& \text { where } f \text { is the transform }
\end{aligned}
$$

variable and lower-case function names denote Fourier transforms, and $t(-f)$ is the conjugate transform of $T(x)$.

The c.c.f. may be calculated most efficiently by using the Fast Fourier Transform (FFT) algorithm and performing the cross-correlation in the Fourier domain.

For ease of computing, each spectrum was normalised to a mean value of zero, thus avoiding the effects of a large zero-frequency component in the transform. The ends of each spectrum were then attenuated with a cosine bell applied to the first and last ten percent of the data train in order to minimise end effects. The length $\mathrm{N}$ of the array containing the spectrum was required to be a power of 2 by the FFT algorithm used.

Because the sections of spectrum used for the correlation behave as periodic functions when represented by finite Fourier series, problems can arise due to the end of the "shifted" spectrum overlapping the start of the template spectrum and vice versa. To overcome this effect, the data arrays were doubled in length to $2 \mathrm{~N}$ pixels, and the extra $N$ locations at the end of each array were set at zero.

After "masking" as described above, the transforms of the spectra were calculated. An optimum high frequency filter of the type devised by Brault and White (1971) was applied to each spectrum in the Fourier domain.

The filtered transforms were then correlated using 
Eq. 3-4 and the inverse transform of the result was computed. The c.c.f. obtained for each spectrum was a periodic function, with a period of $\mathrm{N}$ pixel units. The peak of the c.c.f.' was displaced from the origin by a number of pixel units equal to the lag between the program and template spectra. The lag was measured by fitting a Gaussian to the c.c.f. peak by least squares, and determining its centre; in cases where the lag was negative, the displacement was measured from the end of the array.

The lags were then converted from pixel units to kilometres per second, and heliocentric corrections for the radial velocities of both the program star and the template star were applied

$$
\begin{aligned}
& \mathrm{V}_{\mathrm{H}}=\mathrm{v}_{\mathrm{CCf}}+\mathrm{v}_{\mathrm{t}}+\Delta \mathrm{v}_{\mathrm{H}}+\Delta \mathrm{v}_{\mathrm{t}}
\end{aligned}
$$

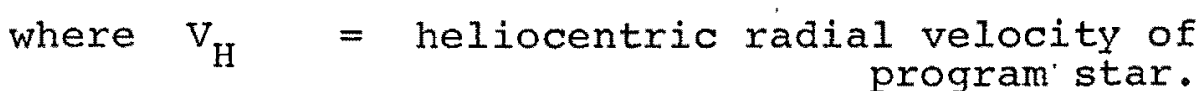

$$
\begin{aligned}
& v_{t}=\text { heliocentric radial velocity of } \\
& \mathrm{V}_{\mathrm{CCf}}=\mathrm{c} \cdot \mathrm{c} \cdot \mathrm{f} \cdot \operatorname{lag}\left(\text { in } \mathrm{kms}^{-1}\right) \text {. } \\
& \Delta \mathrm{V}_{\mathrm{H}}=\text { heliocentric correction for } \\
& \text { program star. } \\
& \Delta v_{t}=\text { heliocentric correction for } \\
& \text { template star }
\end{aligned}
$$

In order to assess the accuracy of the method, several observations were made of bright stars with we11-determined radial velocities (Griffin, 1972).

The template star used in all cases was HR 141 (KO III; $v_{t}=18.7 \pm 0.2 \mathrm{~km} \mathrm{~s}^{-1} ;$ Griffin, 1972). The results for these observations of radial velocity standard stars are summarised in Table 3.1. 
Table 3.1

\begin{tabular}{|c|c|c|c|c|c|c|}
\hline $\begin{array}{c}\text { Star } \\
\text { HR }\end{array}$ & $\begin{array}{l}\text { Radial } \\
\text { Velocity }\left(\mathrm{kms}^{-1}\right) \\
\text { (Griffin, I972) }\end{array}$ & $\begin{array}{l}\text { HJD } \\
\text { (mid-exp) } \\
2444000+\end{array}$ & $\begin{array}{l}\text { C.c.f. } \\
\text { lag } \\
(\text { pixel) }\end{array}$ & $\begin{array}{l}\text { Heliocentric } \\
\text { Correction } \\
\left(\mathrm{kms}^{-1}\right)\end{array}$ & $\begin{array}{l}\text { Heliocentric } \\
\text { Radial } \\
\text { Velocity }\left(\mathrm{kms}^{-1}\right)\end{array}$ & $O-C$ \\
\hline 141 & -18.7 & 566.932 & - & -22.2 & -18.7 & - \\
\hline 387 & -3.0 & 566.939 & 1.44 & -20.6 & -2.7 & 0.3 \\
\hline 406 & -26.8 & 566.947 & -1.12 & -20.1 & -27.9 & 1.1 \\
\hline 507 & +19.7 & 566.955 & 3.49 & -18.4 & +19.9 & 0.2 \\
\hline 1450 & -3.8 & 567.114 & -1.35 & 1.8 & -8.2 & -4.4 \\
\hline 1488 & +34.1 & 567.120 & 2.26 & 3.1 & +29.1 & -5.0 \\
\hline 8897 & +8.9 & 566.916 & 3.18 & -28.3 & +6.9 & -2.0 \\
\hline 8928 & +13.3 & 566.924 & 3.66 & -28.4 & +11.6 & -1.7 \\
\hline
\end{tabular}

Table 3.1. Observed and catalogued radial velocities for eight radial

velocity standard stars, 1980 November. 
The r.m.s. deviation of the observed radial velocities in Table 3.1 from those given by Griffin, is $\pm 2.7 \mathrm{kms}^{-1}$. This figure is indicative of the precision which we may expect for the radial velocities of the program stars.

In practice, however, the uncertainty in the radial velocity as determined from the c.c.f. lag is dependent on the width of the c.c.f. peak. Many of the stars observed were found to have projected equatorial rotation speeds in excess of $v_{e} \sin i=20 \mathrm{kms}^{-1}$.

The resulting smearing of the stellar spectrum also affects the cross correlation function. It may be seen from the definition of the c.c.f. in Eqs. 3-3 and 3-4 that convolution of the spectrum $G(x)$ with a rotational smearing profile $S(x)$ will give

$$
c(f)=g(f) . s(f) \cdot t(f)
$$

in the Fourier domain. The resulting c.c.f. is simply the convolution of the "non-rotating" c.c.f. with the rotational smearing profile of the program star.

The c.c.f. for a double-lined spectroscopic binary will have twin peaks, whose shifts from the origin give the individual radial velocities of the binary components. In this case particularly, the cross-correlation method has the advantage over more conventional radial velocity techniques, that individual lines need not be identified as belonging to one component or the other prior to evaluating the radial velocities. The spectrum is analysed as a whole, and the problems of blended lines in overlapping spectra are effectively eliminated.

The results of the $\mathrm{Mg}$ I b radial velocity survey are discussed for individual stars in Chapter 4. 


\section{$3.4 .1 \cdots$ Preliminary Treatment}

Fixed-pattern noise in the spectra was removed by flat-fielding, using the technique described in section 3.3 .1 .

\subsubsection{Wavelength Calibration}

The spectra were calibrated using a third-order polynomial fit to the wavelengths of eight strong telluric $\mathrm{H}_{2} \mathrm{O}$ absorption lines seen superimposed on the stellar spectra.

This method was used in preference to an arc spectrum calibration, owing to the lack of a sufficient number of neon-iron or argon-iron arc lines near Ho at the high dispersion used. The r.m.s. deviation of the known $\mathrm{H}_{2} \mathrm{O}$ wavelengths from the values derived using the polynomial fit was again about $0.1 \AA$ for all spectra.

\subsubsection{Velocity Reductions}

For those spectra in which the rotational smearing was less than $v_{e} \sin i \sim 30 \mathrm{~km} \mathrm{~s}^{-1}$, the weak metallic lines near $\mathrm{H} \alpha$ were sufficiently well-defined to be used for radial velocity determinations.

In spectra containing a strong $\mathrm{H} \alpha$ absorption feature, the width and strength of the $\mathrm{H} \alpha$ profile would have caused it to dominate the c.c.f. if Simkin's method were applied directly to the "untreated" spectra. To avoid this, the H $\alpha$ feature was artificially excised from all spectra. An artificial section of flat continuum was inserted in its place, thereby ensuring that only the sharp metallic lines contributed to the c.c.f. peak.

Each spectrum was then re-binned into uniform increments in log wavelength, normalised to a mean value of zero and 
masked using the same methods described in section 3.3 .3 for the Mg I b spectra.

The template spectrum used was that of HD 157457

(G8 III; $v_{r}=17.4 \pm 0.2 \mathrm{~km} \mathrm{~s}^{-1}$ ). The template was calibrated using the rest wavelengths of the sharp metallic lines in the stellar spectrum. The c.c.f. lags of the spectra with respect to this template thus gave a direct measure of the geocentric radial velocity for each program star, thereby eliminating the need to apply additional corrections for the template velocity.

The filtering and cross correlations were performed in the Fourier domain, using simkin's method as described in Section 3.3 .3 .

For values of $v_{e} \sin i$ less than about $30 \mathrm{~km} \mathrm{~s}^{-1}$, the metallic absorption lines were sufficiently well-defined that they could be compared with artificially-smeared spectra for various values of $v_{e} \sin i$. The $v_{e} \sin i$ values estimated in this way were uncertain by about $5 \mathrm{~km} \mathrm{~s}^{-1}$.

3.4.4 Continuum Fitting

The primary aim of the $\mathrm{H} \alpha$ survey was a qualitative inspection of the range of emission activity present in the program stars.

The $H \alpha$ spectra accompanying the notes on individual systems in Chapter 4 were produced using a plotting routine incorporated in the Mt. Stromlo SPECT, package. prior to plotting, each raw spectrum was re-binned from the original pixel scale to a wavelength scale in the rest frame of the observer. 
The spectra were then filtered by convolving them with a narrow Gaussian of the form

$$
F(x)=A e^{-\left(\frac{x}{1.2}\right)^{2}}
$$

$$
\text { where } A \text { is a normalisation }
$$

constant and $x$ is in pixel units.

This filter effectively eliminated large-amplitude,highfrequency noise in the spectra, without significant degradation of the line profiles.

Finally, the spectra were flattened and normalised to a continuum level of unity, using continuum windows near $6550,6553,6557,6567,6572$ and $6578 \AA$. 


\subsubsection{Preliminary Treatment}

All spectra were divided by normalised flat-field exposures to remove fixed-pattern detector noise.

In the case of the siding spring 2D PCA data, each spectrum occupied between three and five rows (depending on the seeing conditions during the exposure) of the two dimensional data array. The rows containing the spectrum were flat-fielded separately, and were then added together pixel-by-pixel to produce a single row spectrum. The same procedure was followed for the corresponding rows of the neon arc spectra.

\subsubsection{Wavelength Calibration}

The spectra were calibrated using a third-order polynomial fit to the wavelengths of lines in the neon arc spectra accompanying each stellar spectrum, for both the MSO coudê spectra and the sso échelle spectra.

\subsubsection{Velocity Reductions}

For each spectrum, a section of the absorption spectrum outside the $\mathrm{H}$ and $\mathrm{K}$ absorption line centres and containing as many strong, well-defined absorption lines as possible was chosen. In the case of the sso $k$-line spectra, the region $3916 \AA-3930 \AA$ was used; for the MSO $H$ and $K$ spectra, the region 3900 - $3930 \AA$ was used.

The spectrum of the radial velocity standard star HD $80170\left(v_{r}=0.0 \pm 0.2 \mathrm{~km} \mathrm{~s}^{-1}\right)^{1}$ was used as the template. As with the Mg I b spectra, the wavelength calibration of the template was performed using the neon-iron arc spectrum rather than the stellar spectrum.

1. Trans I.A.U. IX, 442 (1957). 
Filtering and cross-correlation of the program star spectra with the template spectrum was carried out in the Fourier domain using Simkin's method (Section 3.3.3), and heliocentric corrections were applied to the resulting radial velocities.

In addition, the radial velocities of the $\mathrm{H}$ and $\mathrm{K}$ emission peaks were measured. A Gaussian was fitted to each line core in order to determine the position of its centre, and the radial velocity was measured directly from the observed line wavelength and the rest wavelength. Heliocentric corrections were again applied.

\subsubsection{Surface gravities using the Wilson-Bappu effect}

The empirical relationship between the absolute visual magnitude of a star and the full widths at half maximum (FWHM) of the emission cores in the $\mathrm{Ca}$ II $\mathrm{H}$ and $\mathrm{K}$ lines (Wilson and Bappu, 1957) may be expressed in terms of the more fundamental atmospheric parameters gravity, $g$, and effective temperature, $T_{e}$ (Reimers, 1973; Necke1, 1974).

Ayres (1979) gives the general form of the empirical expression for the FWHM of the $\mathrm{K}$ emission core, $\mathrm{W}_{\mathrm{O}^{\prime}}$, as

$$
w_{0} \sim g^{\alpha} \mathrm{T}_{\mathrm{e}}^{\beta} \mathrm{A}_{\mathrm{Fe}}{ }^{\gamma}
$$

where $\mathrm{A}_{\mathrm{Fe}}$ is the iron abundance relative to the sun, and

$$
\begin{array}{r}
-0.23 \leqslant \alpha \leqslant-0.20 \\
1.3 \leqslant \beta \leqslant 1.7 \\
0.0 \leqslant \gamma \leqslant 0.1
\end{array}
$$

Ayres' analytical investigation indicated that the form of this relation was largely a consequence of the hydrostatic equilibrium conditions in the stellar chromosphere, 
and confirmed that $W_{0}$ should be more or less independent of the degree of chromospheric heating. Similar relationships exist for the separations of the $K_{1}$ minima and the $K_{2}$ peaks in the emission profile; however, both these quantities are dependent on the overall level of chromospheric heating. In order to obtain estimates of the surface gravities of the program stars, it was necessary first of all to calibrate the expression $3.5-1$ using stars with well-determined emission widths, surface gravities and effective temperatures. The rather weak dependence on the metallicity was ignored, owing to the lack of abundance measurements for any of the program stars, and a relation of the form

$$
\log W_{o}(\AA)=-0.22 \log g+1.5 \log \mathrm{T}_{\mathrm{e}}+\text { const. }
$$

was assumed, using intermediate values of $\alpha$ and $\beta$ (Eq.3.5 - 1). The calibration was based on high-resolution Ca II k-line data presented in a paper by Linsky, worden, MCClintock and Robertson (1979). Independently-determined values of $\mathrm{T}_{e}$ and $\log \mathrm{g}$ were given in their paper for fifteen stars with a wide range of spectral types, luminosity classes and absolute $\mathrm{Ca}$ II $\mathrm{H}$ and $\mathrm{K}$ line surface fluxes.

The $W_{0}$ values for the $K$ line were measured by Linsky et al directly from their spectra, and were not corrected for instrumental resolution. They stated, however, that the instrumental profile could be approximated closely by a gaussian with a FWHM of $0.120 \AA$.

An approximate correction for the effect of instrumental degradation on the emission width may be made by treating the emission core as a gaussian. Deconvolution of this profile with the instrumental profile gives a gaussian whose half-width at half-maximum (HWHM) is given by 


$$
\begin{gathered}
\left(\frac{W_{o}}{2}\right)^{2}=\beta_{\text {obs }}^{2}-\beta_{\text {inst }}^{2} \quad(3.5-3) \\
\text { where } \beta_{\text {obs' }} \beta_{\text {inst }} \text { are the }
\end{gathered}
$$

HWHM's of the observed and instrumental profiles respectively. The corrected widths and the values of the constant in relation 3.5-2 obtained for the fifteen stars selected for the calibration, are given in Table 3.2 .

\section{TABLE 3.2}

Star

$$
\begin{aligned}
& { }^{*} \text { FWHM } A \\
& \text { Ca II K }
\end{aligned}
$$$$
\begin{aligned}
& W_{O}=\text { corrected } \\
& \text { FWHM }(\AA)
\end{aligned}
$$$$
{ }^{*} \mathrm{~T}_{\mathrm{e}} \quad * \log 9
$$

$\log w_{0}+0.22 \log g$ Ca II

0.68

0.67

6100

4.4

$-4.88$

HD 76151

0.72

0.71

5600

4.6

$-4.76$

61. UMa

0.49

0.48

5350

4.5

$-4.93$

$\xi B O 0 A$

0.47

0.45

5250

4.4

$-4.95$

70 Oph A

0.51

0.50

5150

4.4

$-4.90$

E $\operatorname{Er} i$

0.40

0.38

5000

4.5

$-4.98$

EQ Vir

0.36

0.34

4000

4.4

$-4.90$

$\alpha$ Boo

0.92

0.91

4250

1.7

$-5.11$

$\alpha$ Tau

0.99

0.98

3800

1.4

$-5.07$

E Leo

1.40

1.39

5300

1.40

$-5.13$

B Dra

2.05

2.05

5375

1.35

$-4.99$

$\varepsilon$ Gem

1.92

1.92

4600

1.85

$-5.02$

$56 \mathrm{Peg}$

1.40

1. 39

4500

1.75

$-4.95$

E Peg

1.76

1.76

4400

1.00

$-5.00$

E CY9

1.80

1.80

4100

1.75

$-4.78$

* Information from Linsky et al (1979).

Table 3.2 Stars used for calibration of empirical

Wilson-Bappu relation. 
The calibrated relation giving $\log g$ as a function of $W_{0}$ and $T_{e}$ is thus

$$
\log g=\frac{1}{0.22} \quad 1.5 \log \mathrm{T}_{\mathrm{e}}-\log \mathrm{W}_{\mathrm{o}}-4.96
$$

The scatter in the relation amounts to a lo uncertainty of \pm 0.50 in the $\log g$ value obtained for any star. The goodness of fit of Eq. (3.5-4) to the independently-determined $\log \mathrm{g}$ values is illustrated in Fig. 3.1.

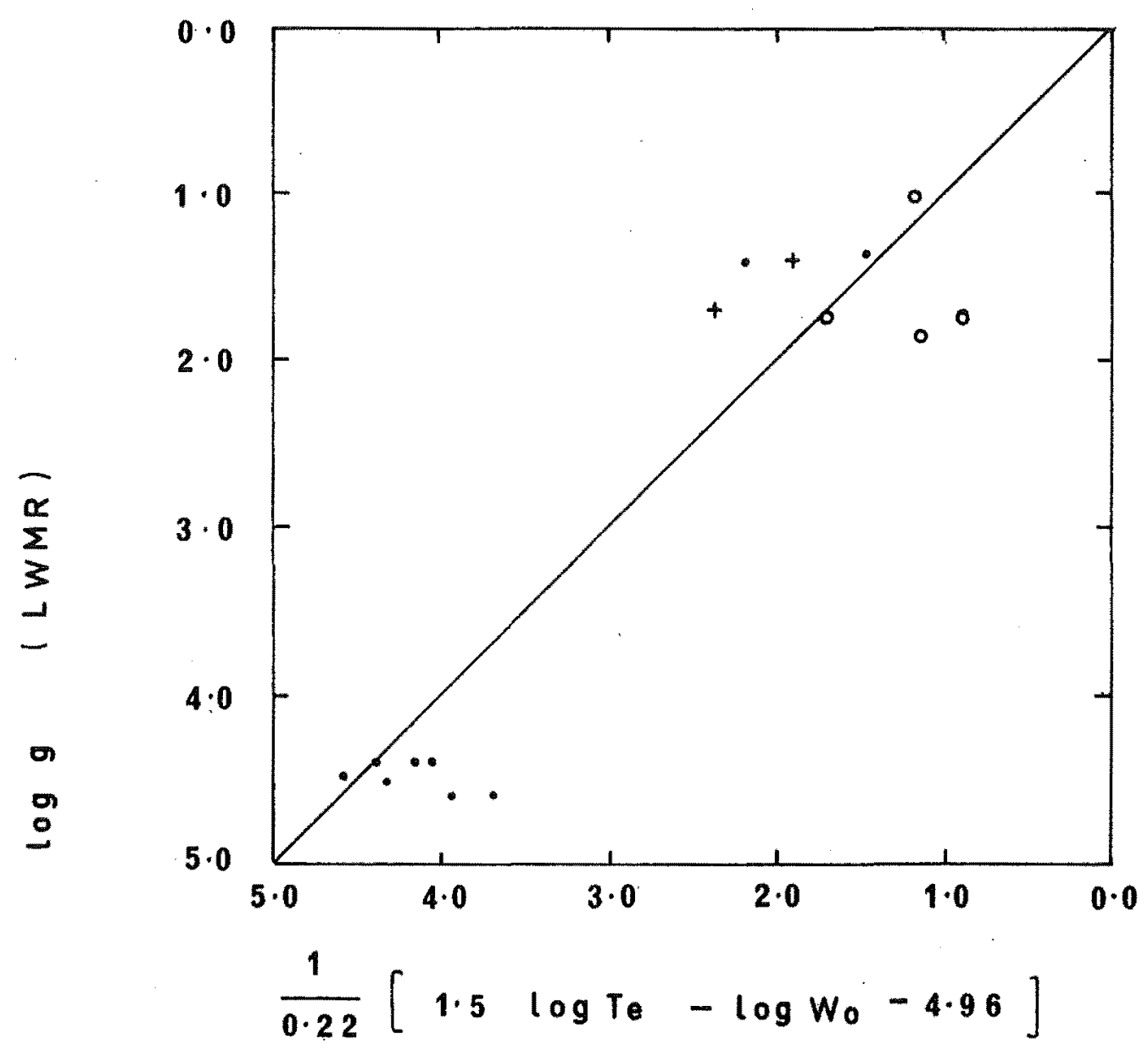

Fig. 3.1

Elot of independently-measured $\log g$ values against values obtained using Eq.3.5-4, for 15 stars listed in Table 3.2. Absolute Ca II emission surface fluxes are given by symbols used:

$$
\begin{array}{ll}
\text { dots }=\geqslant 10^{6} & \text { erg } \mathrm{cm}^{-2} \mathrm{~s}^{-1} \\
\text { circles }=10^{5}-10^{6} & \text { erg } \mathrm{cm}^{-2} \mathrm{~s}^{-1} \\
\text { crosses }=10^{4}-10^{5} & \text { erg } \mathrm{cm}^{-2} \mathrm{~s}^{-1}
\end{array}
$$


The SSO Ca II $\mathrm{K}$ line spectra have an instrumental profile which may be approximated by a gaussian with a FWHM of $0.3 \AA$. A conrection must also be made to the emission core widths, to compensate for the effects of rotational broadening. For simplicity, a gaussian was again used to approximate the effect of rotation, and the value

$$
\begin{array}{r}
\beta_{\text {rot }}=\frac{\sqrt{3}}{2} \lambda \frac{V_{e}}{\mathrm{c}} \sin i(\AA) \\
\text { was adopted for the HWHM of the }
\end{array}
$$

rotational profile. Deconvolution of the rotational and instrumental profiles from the observed emission profile gives

$$
\left(\frac{W_{o}}{2}\right)^{2}=\beta_{\text {obs }}^{2}-\beta_{\text {inst }}^{2}-\beta_{\text {rot }}^{2}
$$

The values of $v_{e} \sin i$ were estimated from the amount of rotational smearing of the $\mathrm{H} \alpha$ spectra (Section 3.4.3). Theoretical profiles for the weak metallic lines near $\mathrm{H} \alpha$ were calculated for various values of $v_{e}$ sin $i$, and were compared with the observed profiles.

Where values of $v_{e} \sin i$ in excess of $30 \mathrm{~km} \mathrm{~s}^{-1}$ were encountered, the metallic line spectrum near $\mathrm{H} \alpha$ was smeared to such an extent that it became lost in the noise. In these cases, the value of $v_{e}$ sin $i$ was estimated in a similar way, using theoretical smearing profiles calculated for the crosscorrelation functions of the Mg I b spectra (Section 3.3.3). The results for the program stars observed at siding spring are summarised in Table 3.3 . 
TABLE 3.3

\begin{tabular}{|c|c|c|c|c|c|}
\hline $\begin{array}{l}\text { Star } \\
\text { HD }\end{array}$ & $\begin{array}{l}\text { FWHM ( }(\AA) \\
\text { Ca II K }\end{array}$ & $\begin{array}{l}v_{e} \sin i \\
\left(k m s^{-1}\right)\end{array}$ & $\begin{array}{l}W_{0} \\
(\AA)\end{array}$ & $\begin{array}{l}T_{e} \\
(\mathrm{~K})\end{array}$ & $\log g$ \\
\hline 46697 & $0.98 \pm .05$ & $10 \pm 3$ & $0.90 \pm .05$ & $4600 \pm 200$ & $2.6 \pm 0.5$ \\
\hline 81410 & $0.81 \pm .05$ & $15 \pm 5$ & $0.67 \pm .05$ & $4700 \pm 200$ & $3.3 \pm 0.5$ \\
\hline 101309 & $0.74 \pm .05$ & $15 \pm 3$ & $0.58 \pm .05$ & $4700 \pm 200$ & $3.6 \pm 0.5$ \\
\hline *101379 & $1.4 \pm .2$ & $10 \pm 3$ & $1.3 \pm \ldots 2$ & $4000 \pm 200$ & $1.4 \pm 0.7$ \\
\hline 119285 & $0.71 \pm .05$ & $\leqslant 8$ & $0.64 \pm .05$ & $4600 \pm 200$ & $3.3 \pm 0.5$ \\
\hline 127535 & $0.83 \pm .05$ & $15 \pm 5$ & $0.69 \pm .05$ & $4600 \pm 200$ & $3.2 \pm 0.5$ \\
\hline+137164 & $\leqslant 0.75 \pm .05$ & $\leqslant 8$ & $\leqslant 0.69 \pm .05$ & $4500 \pm 200$ & $\geqslant 3.1 \pm 0.5$ \\
\hline 165141 & $0.85 \pm .05$ & $\leqslant 8$ & $0.80 \pm .05$ & $4600 \pm 200$ & $2.9 \pm 0.5$ \\
\hline 181809 & $0.79 \pm .05$ & $\leqslant 8$ & $0.73 \pm .05$ & $4600 \pm 200$ & $3.0 \pm 0.5$ \\
\hline
\end{tabular}

* FWHM from MSO coudé spectra obtained 1981 March 24-26, at lower dispersion but same resolution.

+ Emission from both components superimposed.

Table 3.3 Ca II $\mathrm{K}$ emission widths and surface gravities for program stars observed at SSO, 1981 April 13-20. 
$3.5 .5 \mathrm{Ca} I I \mathrm{H}$ and $\mathrm{K}$ line surface fluxes

The calibration method developed by Linsky et al (1979) (hereinafter referred to as IWMR) was used to convert the integrated emission fluxes in the $\mathrm{H}$ and $\mathrm{K}$ lines to absolute fluxes (in erg $\mathrm{cm}^{-2} \mathrm{~s}^{-1}$ ) at the stellar surface.

In their paper, LWMR used $50 \AA$ bandpass photometry by Willstrop (1964) of the region $3925-3975 \AA$ in 81 stars with $\left(V-R_{J}\right)>0.2$. The Barnes-Evans relationship (Barnes and Evans, 1976 ; Barnes, Evans and Parsons, 1976 ; Barnes, Evans and Moffet, 1978) was used to determine the angular diameters of the willstrop stars from their absolute visual magnitudes and $\left(V-R_{J}\right)$ colour indices. The absolute surface fluxes were calculated using the relation

$$
\begin{aligned}
& F(\Delta \lambda)=f(\Delta \lambda)\left(\frac{d}{R}\right)^{2}=f(\Delta \lambda) \quad\left(4.125 \times 10^{8} / \phi^{\prime}\right)^{2}(3.5-7) \\
& \text { where } \begin{aligned}
F(\Delta \lambda)= & \text { absolute surface flux in willstrop } \\
& \text { band } \\
f(\Delta \lambda)= & \text { flux received at Earth in willstrop band } \\
d= & \text { stellar distance } \\
\mathrm{R}= & \text { stellar radius } \\
\phi^{\prime}= & \text { stellar angular diameter in milliarcsec. }
\end{aligned}
\end{aligned}
$$

The Barnes-Evans relations may be written as

$$
\begin{array}{r}
\log \phi^{\prime}=0.4874-0.2 V_{0}+0.858\left(V-R_{J}\right) \quad(3.5-8 a) \\
0.00 \leqslant\left(V-R_{J}\right) \leqslant 1.26 \\
\log \phi^{\prime}=0.7674-0.2 V_{0}+0.640\left(V-R_{J}\right) \\
1.26 \leqslant\left(V-R_{J}\right) \leqslant 4.20
\end{array}
$$

where $V_{0}$ is the apparent visual magnitude and $\left(V-R_{J}\right)$ is the Johnson $(V-R)$ colour index. Both are corrected for inter-stellar extinction and reddening. 
A direct calibration can thus be made of Willstrop's data against the $\left(V-R_{J}\right)$ colour index. LWMR found that an accurate piecewise linear least squares fit to the data could be obtained, independent of luminosity class and having the form

$$
\begin{aligned}
\log F(\Delta \lambda)=8.264-3.076\left(V-R_{J}\right) \\
\left(V-R_{J}\right)<1.30 \\
\log F(\Delta \lambda)=5.500-0.944 \begin{array}{l}
\left(V-R_{J}\right) \\
\left(V-R_{J}\right)>1.30
\end{array}
\end{aligned}
$$

The accuracy of the calibration is dependent on systematic errors in the Willstrop fluxes and the BarnesEvans relations, on the accuracy of $\left(V-R_{J}\right)$ obtained for a program star, on random noise in the spectra and on the intrinsic scatter of $F(\Delta \lambda)$ about the mean relations (3.5-9). The observed scatter, according to LWMR, is about 1.4 용.

of the Ca II spectra obtained during 1981 March and April, only the MSO coudé spectra covered a sufficient wavelength range to apply the LWMR calibration method described above.

The change in detector response with wavelength over the $120 \AA$ range of the spectra was small, and was adequately compensated for during flat-fielding (Section 3.5.1): No other adjustments to the continuum level were made.

The relative emission flux measures were calculated by dividing the integrated fluxes between the $\mathrm{K}_{1}$ and $\mathrm{H}_{1}$ minima by the total integrated flux in the wavelength range 3925 - $3975 \AA$ in the rest frame of the star. The variation in the relative fluxes obtained for a single star on different nights was typically about 10\%. This figure is probably indicative of the random errors in the measurements, although intrinsic variations in the emission strength may have 


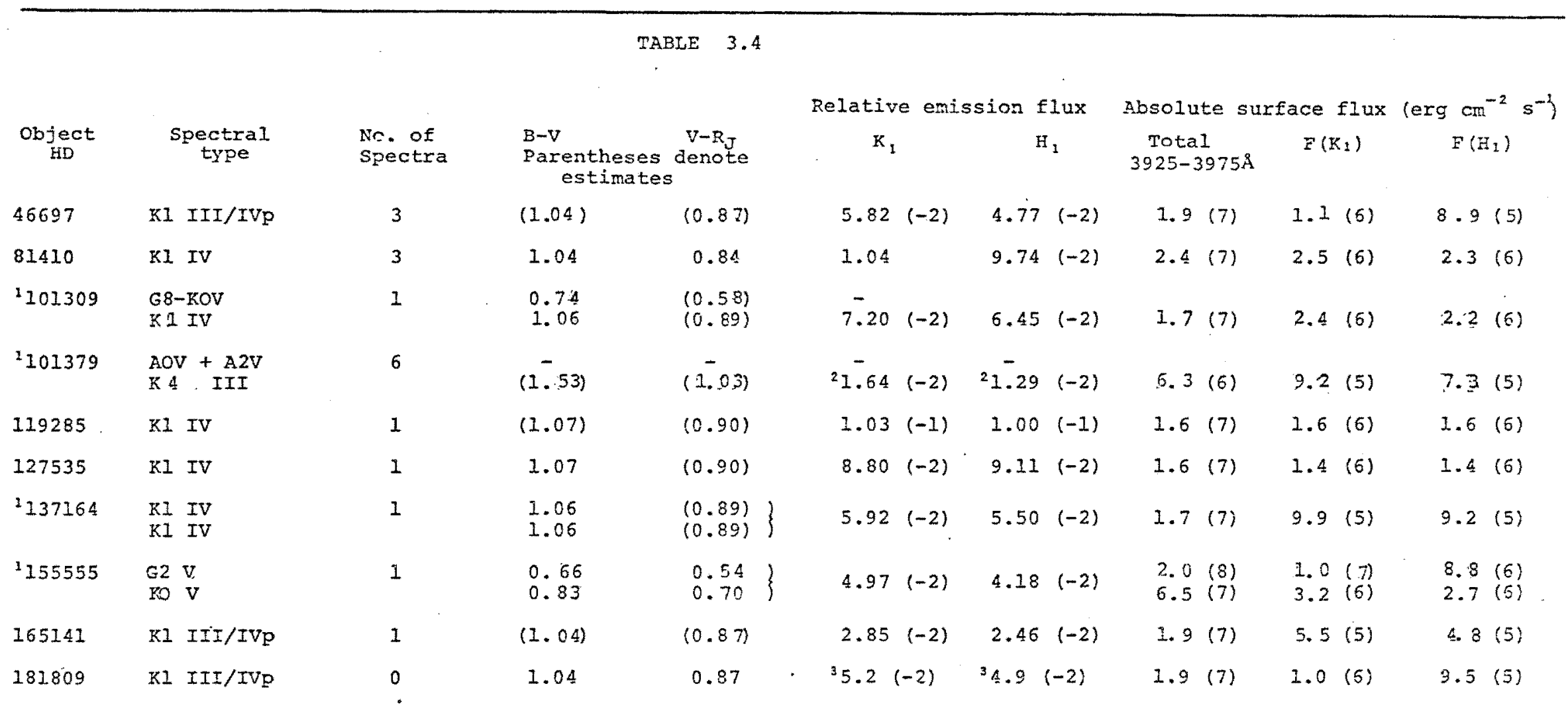

Notes : 1. Composite spectrum. For notes on methods used to cbtain surface fluxes, see Chapter 4.

2. Flux due to underlying A spectrum subtracted from these figures.

3. Flux estimated from 1981 sso data, using $\mathrm{K}$ line ratio HD 31410 : HD $181809=2: 1$.

Table 3.4 Absolute surface fluxes for 1981 March MSO coudé spectra. Figures in parentheses

in colums 6 through 10 denote powers of ten, e.g. $3.0(7)=3 \times 10^{7}$ 
contributed significantly.

$\left(V-R_{J}\right)$ was obtained for each of the stars, by either

(a) direct conversion from BVRI photometry obtained in the Cousins (1976) version of the Cape system (Chapter 2) ;

(b) estimation from (U-B) and (B-V) colour indices obtained during the concurrent UBV photometric program at MSO.;

(c) estimation from known spectral type and the relations of Johnson (1966).

With this information, the absolute flux in the willstrop band could be obtained using equations 3.5-9, permitting a direct conversion of the flux in the $\mathrm{H}$ and $\mathrm{K}$ emission cores to absolute surface flux units. The results are presented in Table 3.4 .

Complications arose in the cases of HD 101309, 101379, 137164 and 155555, where more than one stellar spectrum contributed to the flux in the willstrop band. The figures for these stars given in Table 3.4 were calculated for the chromospherically-active star in each system; the corrections applied to obtain these results are described fully for each individual system in Chapter 4.

3.5.6 Measurement of $\mathrm{Ca}$ II emission flux variations

The sixty-seven spectra obtained at sso during 1981 April 13-20 provided the opportunity to seek evidence of rotational modulation of the Ca II $K$ emission flux, in those program stars for which orbital and/or rotation periods had been establi shed (Section 3.7).

Although the narrow wavelength range $\left(3915\right.$ - $3955^{\prime} \AA$ ) of the spectra did not permit an absolute calibration in surface flux units using the LWMR method, a similar technique 
was employed to measure variations in the emission core flux, relative to the surrounding absorption spectrum.

In order to exclude the contributions of the underlying absorption spectrum and the emission wings, the following technique was adopted

(a) The total integrated emission flux between the $K_{1}$ minima was measured.

(b) A parabola was fitted to the central part of the $K$ absorption trough, immediately outside the $\mathrm{K}_{1}$ minima. The emission core flux was defined as the integrated flux lying between the $K_{1}$ minima and above the fitted parabola (represented by the shaded area in Fig. 3.1).

(c) The total integrated flux in a band extending $10 \AA$ to either side of the line centre was measured, and the contribution of the emission core (see above) was subtracted. The resulting quantity is represented by the hatched area in Fig. 3.2.

(d) The relative flux ratio

(K emission core flux)

(Total flux in $20 \AA$ band) - (K emission core flux) was evaluated.

The relative flux ratios obtained for the program stars during 1981 April at sso are listed in Table 3.5 and plotted in Fig. 3.3 .

Although the positions of the $K_{1}$ minima were determined subjectively using an interactive plotting routine, it was found that repeated measurements of a single spectrum produced relative fluxes which were consistent to within $5 \%$.

No significant variation in the flux level was seen for HD $46697,119285,165141$ or 181809, with the exception of the 


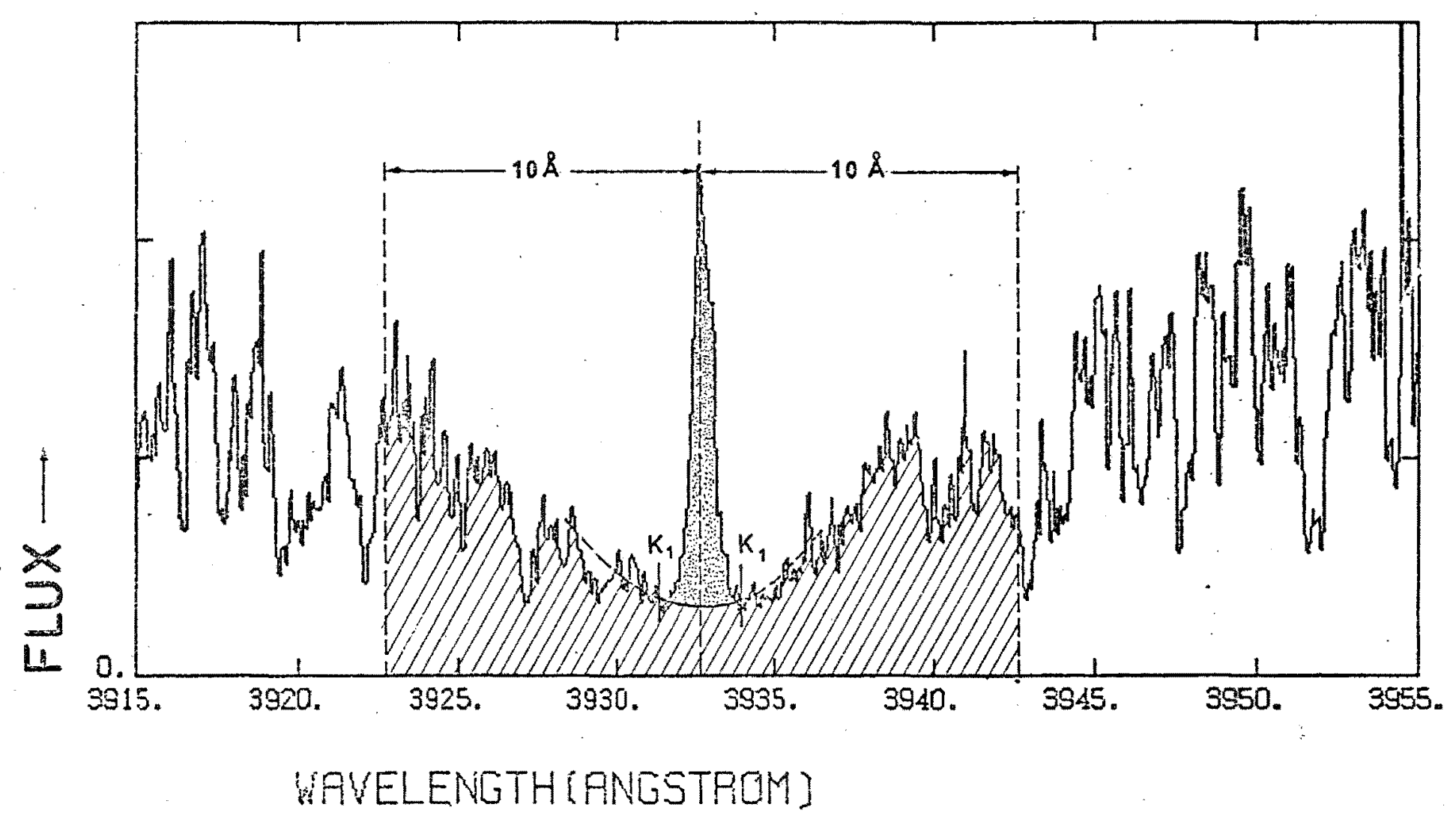

Fig. 3.2 Schematic representation of the method used for monitoring the Ca II $K$ core emission strength in the 1981 April sso spectra. The relative fluxes derived in section 3.5 .6 are given by the ratio of the area of the shaded region to that of the hatched region. 
TAMLE 3.5

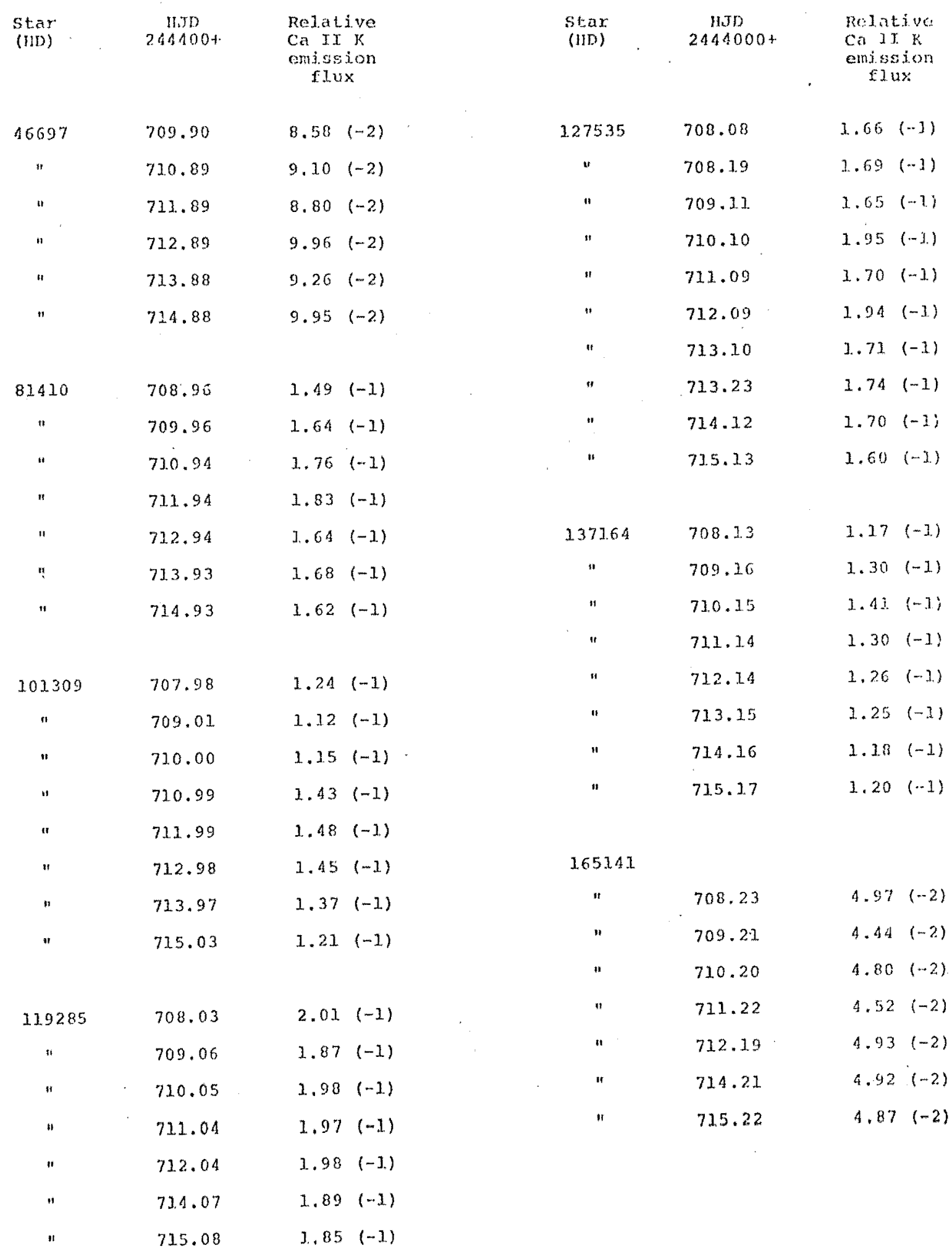

continuod page 69 
TABLE: 3.5 continued

\begin{tabular}{|c|c|c|}
\hline $\begin{array}{l}\text { stax } \\
\text { (Im) }\end{array}$ & $\begin{array}{c}\text { HJW } \\
24410004\end{array}$ & $\begin{array}{l}\text { Relative } \\
\text { CaII } \\
\text { omission } \\
\text { flus: }\end{array}$ \\
\hline 181309 & 709.26 & $8.73(-2)$ \\
\hline$"$ & 710.28 & $1.11(-1)$ \\
\hline$"$ & 711.27 & $8.86(-2)$ \\
\hline$"$ & 712.24 & $8.44(-2)$ \\
\hline$"$ & 713.28 & $8.70(-2)$ \\
\hline$"$ & 714.26 & $8,25(-2)$ \\
\hline$"$ & 715.27 & $8.04(-2)$ \\
\hline
\end{tabular}

Table 3.5 Ca II $\mathrm{K}$ entission core fuxes measured relative to fiux in band $3923,7-3943 \AA$. 
(Ca II $K$ EMISSION CORE FLUX)/(FLUX IN BAND 39237-3943.7 A)

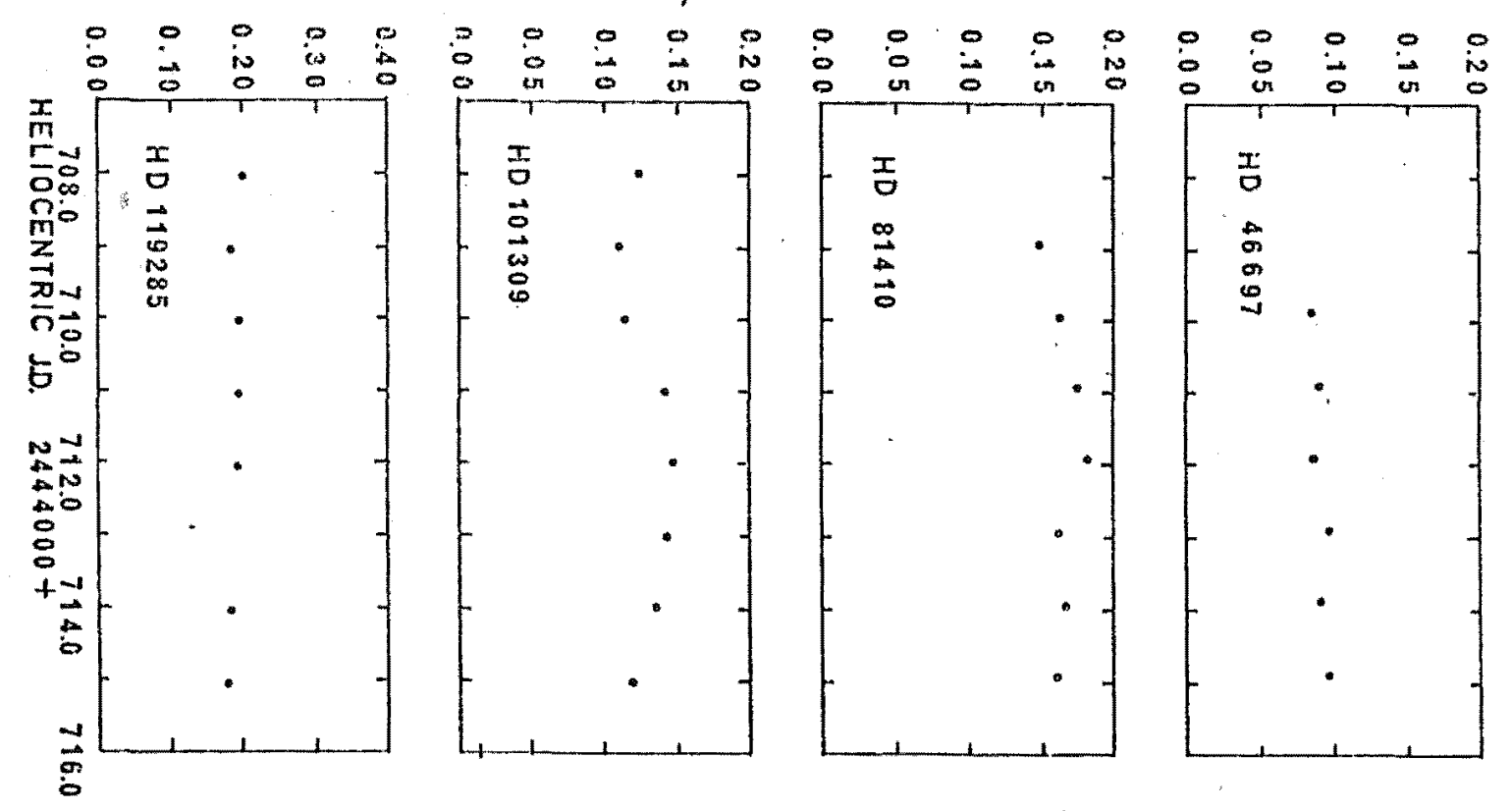

(CaII EMISSION CORE FLUX)/ (FLUX IN BAND 3923.7-3943.7 A)

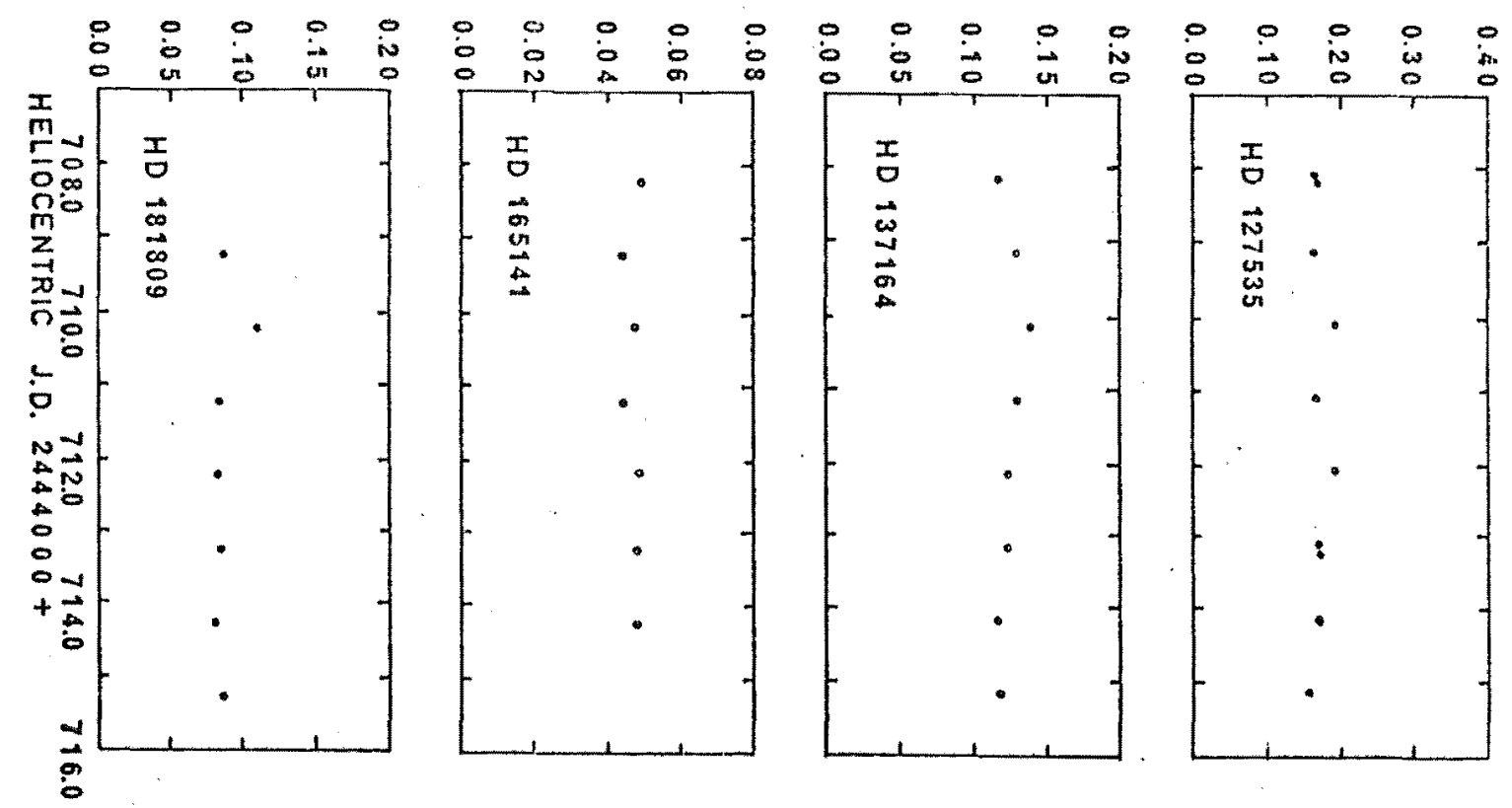

Fig. 3.3

Variations in Ca II $K$ core emission strength in eight stars monitored at sso during 1981 April 13-20. 
single increase seen at HJD 2444710.28 in HD 181809. The high flux level appears to be genuine, and may represent a short-duration flare event.

HD $81410,101309,127535$ and 137164 al1 exhibit continuous variations in their relative fluxes, with total amplitudes of $20,30,20$ and 20 per cent of the mean flux level respectively.

These results are discussed more fully in chapter 4.

3.6 DATA REDUCTION : UBV PHOTOMETRY AT MSO

All photometric data were reduced on the PDP/II computer in the $0.76 \mathrm{~m}$ telescope dome at MSO.

A program was written in BASIC to reduce the data using the methods described in section 2.3. Mean extinction coefficients were used for most nights, based on values obtained on the nights of 1981 March 1, April 8 and May 11 U.T., when extensive observations of stars in the Harvard E-regions were made. Transformation coefficients and zero point constants were evaluated using the same data. All observations of the program stars were transformed to the standard UBV system.

The results for the program stars are presented in Chapter 4 .

\subsection{PRELIMINARY RADIAL VELOCITY SOLUTIONS}

The combined radial velocity data from the $\mathrm{Mg} I \mathrm{~b}, \mathrm{H} \alpha$ and Ca II surveys were in several cases sufficient for periodfinding techniques to be applied and preliminary radial velocity solutions to be obtained.

The method chosen was that described by Gieseking (1981), 
which has been summarised in Section 2.4.4. Circular orbits were assumed, and a sinuosidal least-squares fit to the data was calculated for a range of trial periods. In most cases, a plot of the r.m.s. deviation against $\log \mathrm{P}$ over the range $-0.5 \leqslant \log \mathrm{P} \leqslant 1.5$ revealed a welldefined minimum at the orbital period. Some aliasing was present (see Section 2.4.4) but no serious ambiguities arose where sufficient data was available. The results obtained for individual systems are presented in detail in Chapter 4; a summary of the radial velocity solutions obtained is also given in Table 3.6 .

TABLE 3.6

\begin{tabular}{|c|c|c|c|c|c|c|}
\hline $\begin{array}{l}\text { Object } \\
\text { HD }\end{array}$ & $\begin{array}{c}\mathrm{T}_{O} \\
\text { (HJD } \\
2444000+\text { ) }\end{array}$ & $\begin{array}{c}\mathrm{P} \\
\text { (days) }\end{array}$ & $\left(\mathrm{km}^{\gamma} \mathrm{s}^{-1}\right)$ & $\left(\mathrm{km}^{\mathrm{K}} \mathrm{s}^{-1}\right)$ & $\begin{array}{c}\mathrm{r} . \mathrm{m} . \mathrm{s} . \\
\mathrm{dev} \\
\left(\mathrm{km} \mathrm{s}^{-1}\right)\end{array}$ & $\begin{array}{l}f(m) \\
\left(M_{\odot}\right)\end{array}$ \\
\hline 46697 & 585.56 & $13.61 \pm 0.04$ & 13.1 & 16.4 & 4.3 & $6.2 \times 10^{-3}$ \\
\hline 81410 & 599.70 & $12.9054 \pm 0.0002$ & -10.1 & 40.7 & 4.3 & $9.0 \times 10^{-2}$ \\
\hline 101309 & 597.60 & $11.68+0.02$ & 8.5 & $\begin{array}{l}38.7 \\
39.0\end{array}$ & $\begin{array}{l}4.8 \\
2.5\end{array}$ & $\begin{array}{l}* 0.29 \\
* 0.28\end{array}$ \\
\hline 101379 & 552.52 & $53.850 \pm 0.007$ & 9.1 & 13.8 & 1.5 & $1.5 \times 10^{-2}$ \\
\hline 119285 & 595.91 & $11.89 \pm 0.02$ & 95.2 & 10.7 & 2.1 & $1.5 \times 10^{-3}$ \\
\hline 127535 & 593.72 & $6.006+0.008$ & 16.8 & 35.9 & 4.4 & $2.9 \times 10^{-2}$ \\
\hline
\end{tabular}

* $\frac{M}{M} \sin ^{3} i$. Component $A$ is the cooler, chromospherically active star. Table 3.6 Preliminary radial velocity solutions for six systems. $\quad T_{0}$ is the time of maximum radial velocity for the chromospherically-active component in each system. 


\section{RESULTS FOR INDIVIDUAL SYSTEMS}

$\underline{4.1}$ HD 5303

HD 5303 was first identified as a member of the RS CVn class by Hearnshaw and oliver (1977). The star was classified as a Bamberg variable (BV 625) by strohmeier et.al. (1965).

Photoelectric lightcurves obtained during 1979 and 1980 showed the star to be a partially eclipsing binary, with a large-amplitude distortion of the lightcurve outside eclipse (Collier, Hearnshaw and Austin, 1981).

In this section, high-resolution radial velocity observations and $\mathrm{H} \alpha$ spectra of the HD 5303 system are presented, and a more detailed analysis of the lightcurve is performed.

\section{1 .1 Radial Velocity observations}

$10 \AA \mathrm{mm}^{-1}$ spectra obtained at the coudé focus of the $1.88 \mathrm{~m}$ telescope at Mt.Stromlo showed a double absorption spectrum in the region of the Mg I b lines near $5170 \AA$. Cross-correlation of these spectra with a template spectrum of the radial velocity standard star HR 141 showed that the spectra of the binary components were of approximately equal strength at this wavelength. The radial velocities of the spectra are summarised in Table 4.1.1. A typical crosscorrelation function obtained using Simkin's method (Section 3.3.3) for a spectrum obtained near quadrature is illustrated in Fig. 4.1.1. The radial velocities are plotted against orbital phase (determined from the epoch of mid primary eclipse) in Fig. 4.1.2. 
The high degree of rotational smearing in the spectrum of the cooler star caused some difficulty in determining accurate radial velocities, owing to blending of the c.c.f. peaks, except near quadrature. The lack of data around phase 0.75 precludes reliable estimation of the mass ratio; however, a least squares fit to the existing data for the hotter star gives a radial velocity $\gamma=-2 \pm 2 \mathrm{~km} \mathrm{~s}^{-1}$ for the system's centre of mass, and suggests that $q \simeq 1.0$, giving $\mathrm{K}_{\mathrm{h}}=\mathrm{K}_{\mathrm{C}}=92 \pm 2 \mathrm{~km} \mathrm{~s}^{-1}$. The value of $\mathrm{K}_{\mathrm{h}}+\mathrm{K}_{\mathrm{C}}=184 \pm 4 \mathrm{~km} \mathrm{~s}^{-1}$ is wellestablished from the observations near phase 0.25 , and gives as the system mass $\left(M_{h}+M_{C}\right) \sin ^{3} i=(1.81 \pm 0.12) M_{0}$.

\begin{tabular}{|c|c|c|c|c|c|c|}
\hline \multirow{2}{*}{\multicolumn{2}{|c|}{$\begin{array}{c}\text { Date } \\
\text { (U.T.) }\end{array}$}} & \multirow{3}{*}{$\begin{array}{c}\text { Exposure } \\
\text { (sec.) } \\
300\end{array}$} & \multicolumn{4}{|c|}{ TABLE 4.1 .1} \\
\hline & & & \multicolumn{4}{|c|}{$\begin{array}{c}\text { Heliocentric radial } \\
\text { velocity }\end{array}$} \\
\hline \multirow[t]{3}{*}{1980} & oct. 25 & & 537.949 & 48.7 & & -29.0 \\
\hline & $"$ & $"$ & 538.030 & 25.0 & (?) & - \\
\hline & $"$ & $"$ & 538.144 & 9.3 & (?) & - \\
\hline \multirow[t]{8}{*}{1980} & oct. 26 & $"$ & 538.922 & -96.1 & & 90.9 \\
\hline & & $"$ & 538.928 & -93.5 & & 96.2 \\
\hline & & $"$ & 538.953 & $-92 \cdot 3$ & & 87.7 \\
\hline & & 430 & 539.011 & -90.2 & & 88.0 \\
\hline & & 300 & 539.053 & -89.6 & & 79.7 \\
\hline & & $"$ & 539.115 & -85.3 & & 78.3 \\
\hline & & $"$ & 539.185 & -73.9 & & 65.7 \\
\hline & & $"$ & 539.239 & -73.6 & & 52.8 \\
\hline 1980 & Nov. 22 & $"$ & 565.971 & 34.3 & (?) & - \\
\hline \multirow[t]{3}{*}{1980} & Nov 23 & $"$ & 566.979 & -98.2 & & 82.7 \\
\hline & & $"$ & 567.065 & -94.8 & & 83.3 \\
\hline & & $"$ & 567.167 & -78.8 & & 70.3 \\
\hline
\end{tabular}

Table 4.1.1 Radial Velocities of HD 5303 from Mg I b spectra, MSO 1980 . 
Échelle spectra of the $\mathrm{H} \alpha$ region in HD 5303 obtained during 1980 December 18-21 show a well-defined, shallow Ha absorption core whose radial velocity matches that of the hotter star.

The depth of this absorption feature suggests that the two stars are of approximately equal luminosity at this wavelength.

A broad, shallow absorption feature is seen in the spectrum of the cooler component near phases 0.3 and 0.9 (Figs. 4.1.3(b), (c) and (d)). A weak emission feature shifted some $100 \mathrm{~km} \mathrm{~s}^{-1}$ to blueward of $\mathrm{H} \alpha$ in the cooler component is visible at phases 0.92 and 0.96 ; a similar feature may be present in the red wing of the cooler star's profile, but is overlapped by the hotter star's $H \alpha$ absorption. No evidence of an absorption feature at $\mathrm{H} \alpha$ in the cooler sta is seen at phases 0.58 and 0.64 (Figs $4.1 .3($ a) and. (e)): The asymmetry in the wings of the hotter star's $\mathrm{H}_{\alpha}$ absorption in these two spectra suggests that the cooler star is exhibiting a broad emission profile, slightly above the level of the continuum.

The changes in the $\mathrm{H} \alpha$ profile of the cooler star appear to depend on the orbital phase. The evidence is by no means conclusive, but it does lend support to the hypothesis that the localised flare activity giving rise to the $\mathrm{H} \alpha$ emission has an uneven distribution in longitude on the cooler star, having its greatest concentration on the hemisphere visible near phases 0.58 and 0.64 . This does not appear to coincide with the photometric wave minimum which between 1979 August and 1980 september was located near phase 0.95 , and no definite conclusion can be drawn concerning any link between the starspot activity and the $\mathrm{H} \alpha$ emission in this star. 


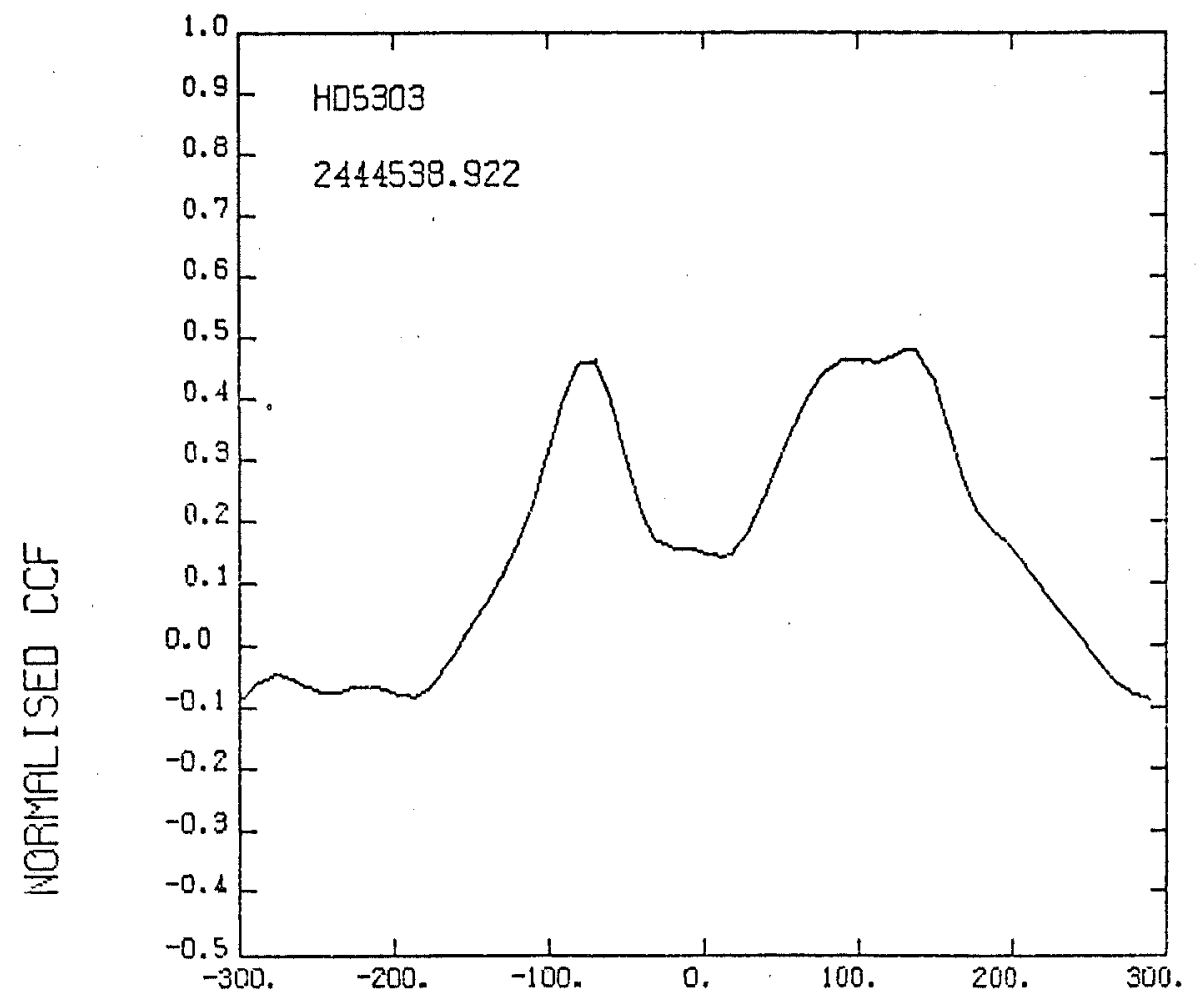

VELOCITY SHIFT $\mathrm{Km} / \mathrm{sec}$

Fig. 4.1.1 Cross-correlation of HD $5303 \mathrm{Mg} \mathrm{I}$ b spectrum with HR 141 template, at orbital phase 0.257. The velocity scale represents the geocentric radial velocity difference between the program star and the template.

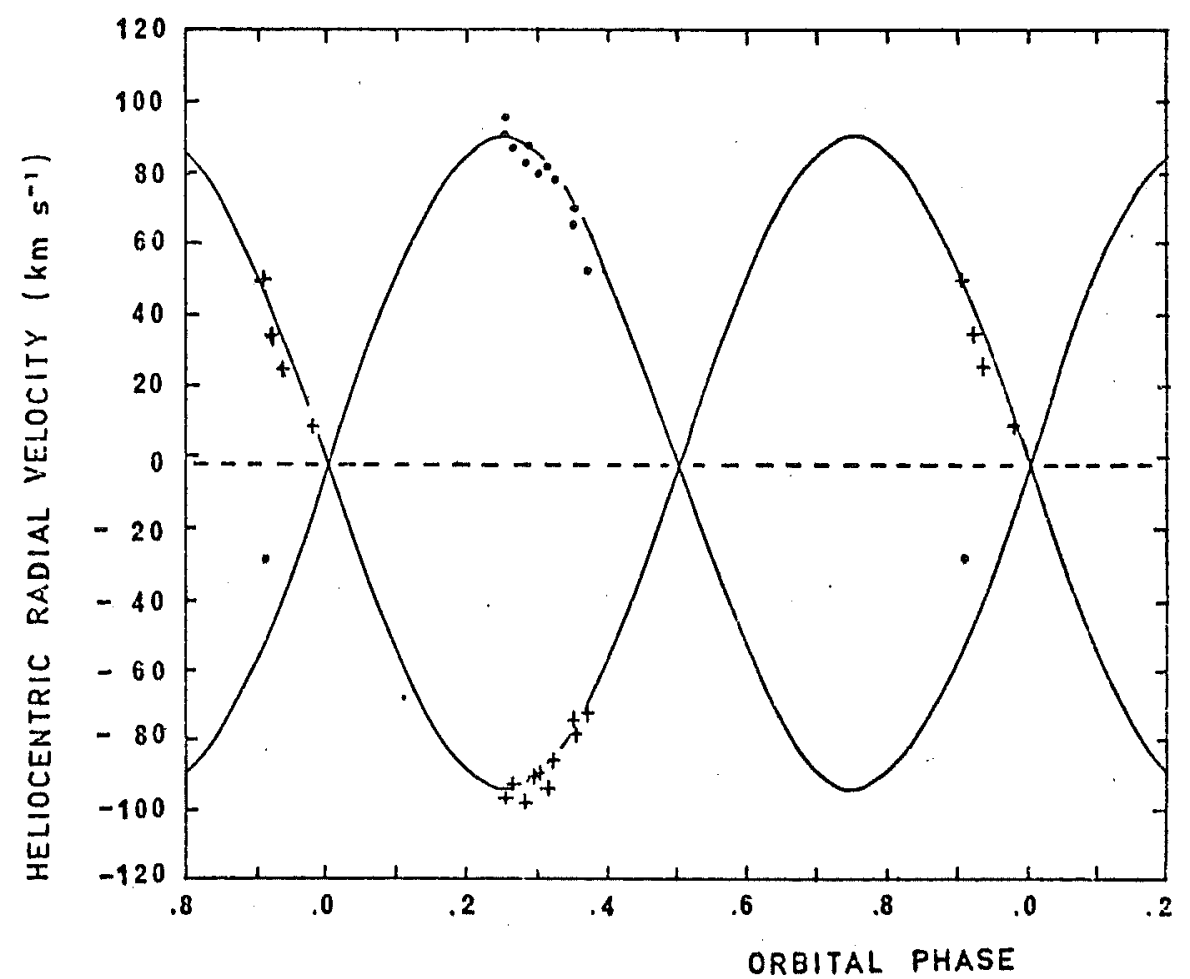

Fig. 4.1.2 Radial velocities of HD 5303, from Mg I b spectra. The ephemeris used was HJD $=2444499.040+2.79786 \mathrm{E}$. 

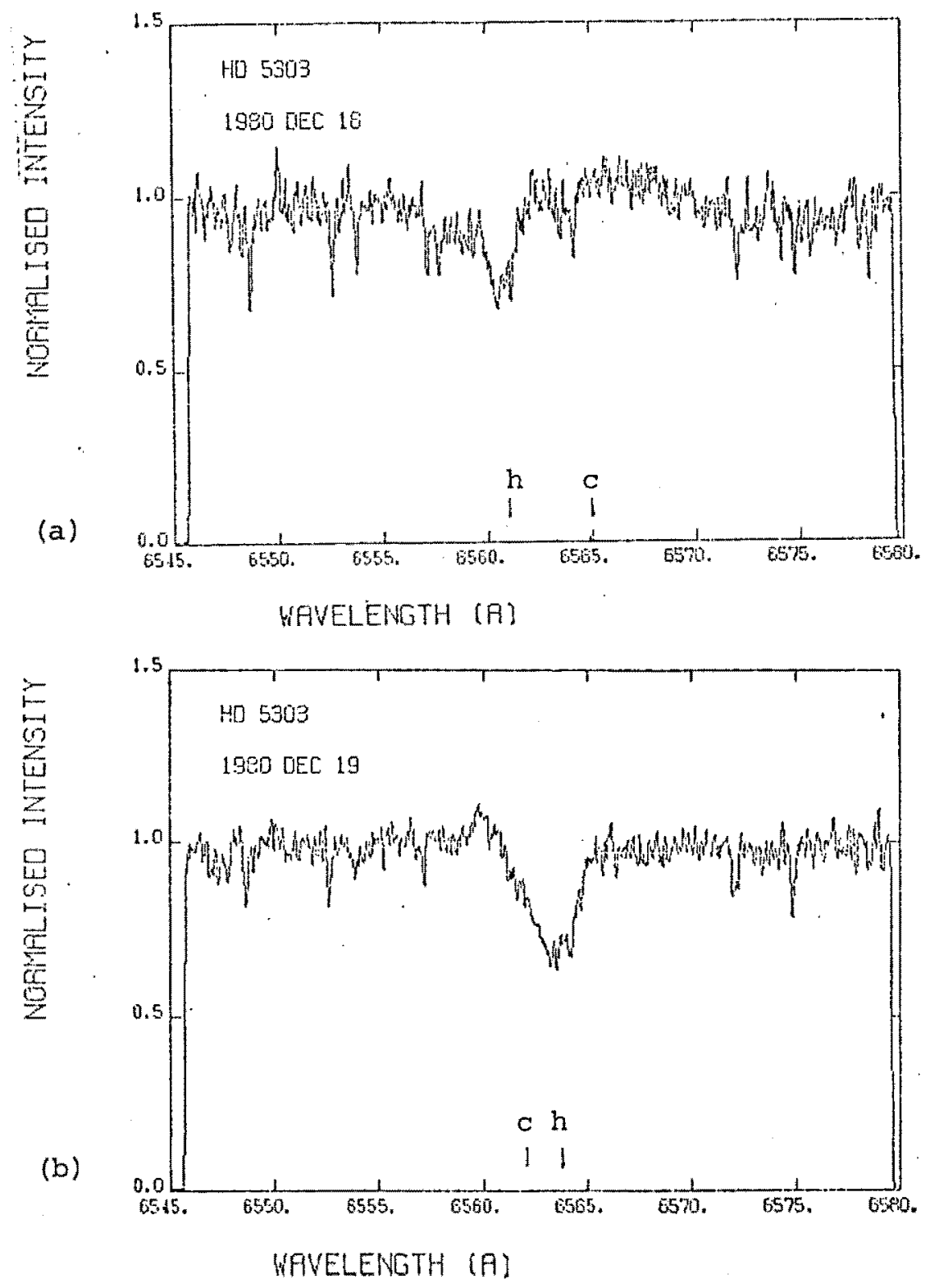

Fig. 4.1.3 H $\alpha$ spectra of HD 5303, 1980 December. Orbital phases are (a) $\phi=0.58$, (b) $\phi=0.92$, (c) $\phi=0.96$, (d) $\phi=0.30$, (e) $\phi=0.64$.

" $h$ " and " $c$ " mark the wavelengths of $H \alpha$ for the hot and cool component, calculated from the radial velocity solution. 

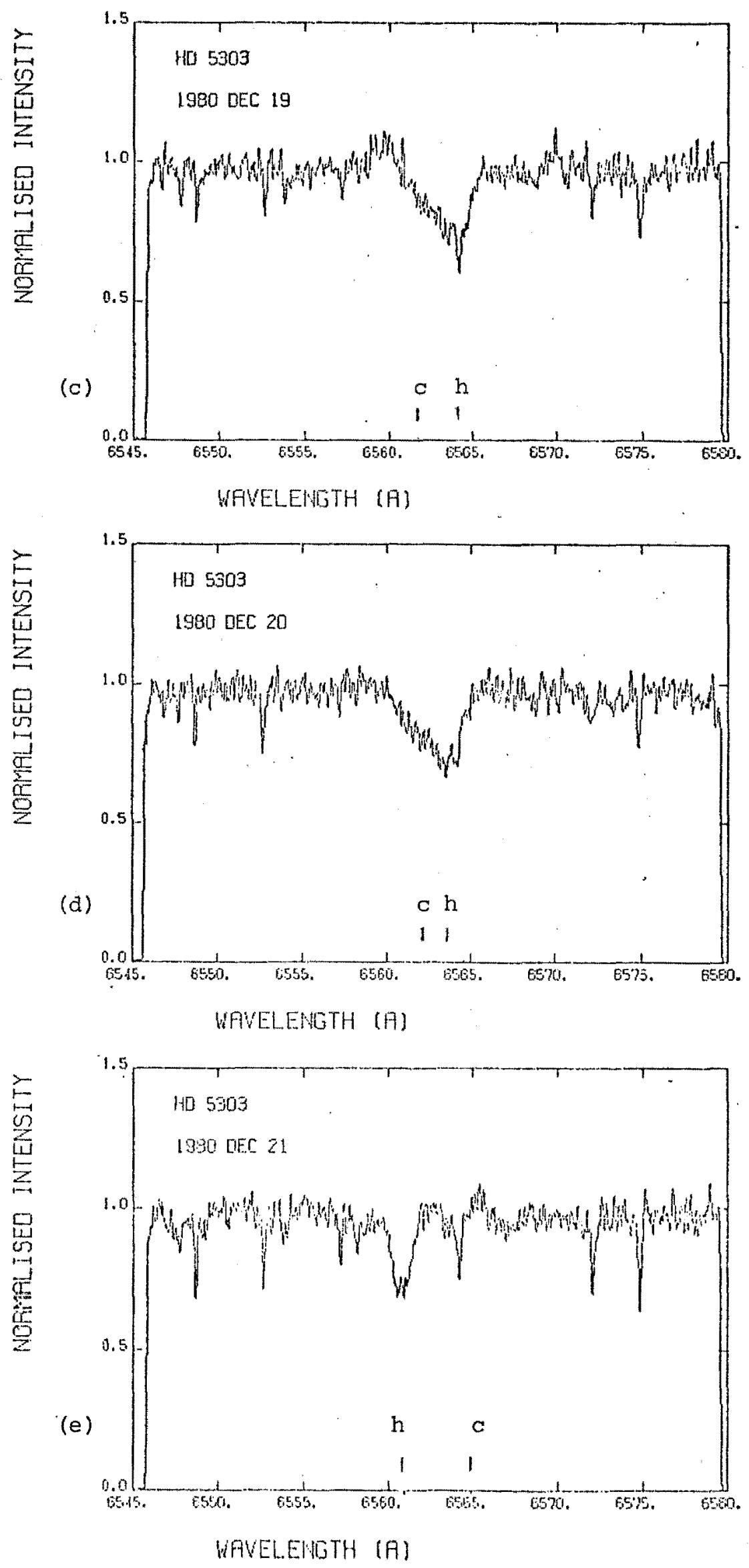

Fig. 4.1.3 (Continued) 
4.1 .3 Further Photometric Analysis

The initial analysis of the out-of-eclipse photometric variation in HD 5303 (Collier, Hearnshaw and Austin, 1981) suggested that tidal distortion of the binary components made a significant contribution to the variation.

Clearly, a more complex model is needed than the Russell model used for the preliminary photometric solution.

The WINK model of Wood $(1972)^{*}$ is well suited to the analysis of detached systems such as HD 5303. However, the difficulties inherent in separating the effects of the spot wave and the tidal distortion preclude a direct iterative solution using the observations alone.

Instead, an approach was adopted in which the colour indices and relative luminosities of the two components were evaluated independently. Initial photometric elements based on this information and on the depth of primary eclipse were then evaluated, using a simple, spherical model.

The WINK program was then run, using these elements, to produce a synthesised lightcurve. The input elements were subsequently adjusted by trial and error until an $(\mathrm{O}-\mathrm{C})$ variation was obtained which showed no discontinuity in slope as the system went into primary eclipse.

The Johnson colour indices of the system at wave maximum are $(B-V)=0.71 \pm 0.01,\left(V-R_{J}\right)=0.64 \pm 0.01$, $\left(V-I_{J}\right)=1.17 \pm 0.01$.

The observed primary eclipse depths are $\Delta V=0.224 \pm$ $0.005 \mathrm{mag}, \Delta(B-V)=0.050 \pm 0.005, \Delta\left(V-R_{J}\right)=0.040 \pm 0.005$ and $\Delta\left(V-I_{\mathrm{J}}\right)=0.080 \pm 0.005$.

The colour indices of the eclipsed portion of the hotter star are thus $(B-V)_{h}=0.52 \pm 0.03,\left(V-R_{J}\right)_{h}=0.45 \pm$ 0.02 and $\left(V-I_{J}\right)_{h}=0.73 \pm 0.03$

\footnotetext{
* With modifications up to and including WINK Status Report No.9 (1978).
} 
The mean relations of Johnson (1966) for the main

sequence thus yield a spectral type of $F 7 / 8 \mathrm{~V}$ and an

effective temperature $\mathrm{T}_{\mathrm{h}}=6110 \pm 110 \mathrm{~K}$ for the hotter star.

The colour indices of the cooler star were evaluated

from the known colours of the system at wave maximum, by varying the luminosity ratio of the components until a set of colour indices was obtained, which were consistent with a single spectral type according to the Johnson relations. Consistency was obtained for a model in which the contribution of the hotter star to the $V$ band luminosity was $(0.60 \pm 0.02)$ of the total.

The corresponding Johnson colour indices of the cooler star were $(B-V)_{C}=1.08 \pm 0.03,\left(V-R_{J^{\prime}}\right)_{C}=0.89 \pm 0.02$ and $\left(V-I_{J}\right)_{C}=1.57 \pm 0.03$, giving a spectral type near $\mathrm{Ko} / 1 \mathrm{IV}$ and an effective temperature $T_{C}=4550 \pm 110 \mathrm{~K}$.

The Barnes-Evans relationship (Barnes, Evans and Moffett, 1978) was next applied to the $V$ luminosity ratio and the $\mathrm{V}-\mathrm{R}_{\mathrm{J}}$ colour indices, yielding a ratio of the angular diameters of the two stars $k=r_{h} / r_{c}=0.42 \pm 0.01$.

A simple spherical model ignoring the effects of limb darkening and ellipticity yielded an orbital inclination $i=67^{\circ} .0 \pm 0^{\circ} .5$, from the observed phases of first and last contact for primary eclipse, the eclipse depth, the surface brightness ratio of the components and the ratio $k$ of their radii.

Several lightcurve synthesis runs using WINK, varying the values of $r_{h} / a$ and $T_{c}$ while holding $i, k$ and $T_{h}$ constant, eventually produced a model which adequately reproduced the observed Iuminosity ratios in the $B, V, R_{J}$ and $I_{J}$ bands, as well as giving a smooth variation in the (O-C) magnitudes, both inside and outside eclipse. 
The photometric elements giving the best fit are given in Table 4.1.2, and the synthesised lightcurves are plotted in Fig. 4.1.4.

The O-C magnitude variations plotted in Fig. 4.1 .5 give the contribution of the spot wave to the variations in HD 5303. The difference between the "unspotted" quadrature magnitude and the observed magnitude outside eclipse in a given band is approximately

$$
\Delta m_{0}=-2.5 \log \left[\frac{1_{h}+1_{c}(1-\alpha(1-\beta))}{1_{h}^{0}+1_{c}^{0}}\right](4.1-1)
$$

where $I_{h} I_{c}$ are the apparent luminosities of the components at the time of observation, with the effects of the spots removed; $I_{h}^{\circ}$ and $1_{\mathrm{c}}^{\circ}$ are the luminosities at quadrature; $\alpha$ is the apparent fraction of the cooler star's visible hemisphere occupied by starspots; and $\beta$ is the ratio of the surface brightness of the spot region to that of the surrounding photosphere.

The "unspotted" out-of-eclipse variation produced by the WINK model may be written simply as

$$
\Delta m_{c}=-2.5 \log \left[\frac{1_{h}+1_{c}}{1_{h}^{\circ}+1_{c}^{\circ}}\right]
$$

The O-C variation is then

$$
\Delta m_{0}-\Delta m_{c}=-2.5 \log \left[1-\frac{\alpha 1_{c}(1-\beta)}{1_{h}+1_{c}}\right] \text {. }
$$

As the ratio $1_{c} /\left(I_{h}+I_{c}\right)$ is effectively constant, both during and outside eclipse, the (O-C) variation is effectively a function only of the apparent spot area $\alpha$ and the surface brightness ration $\beta$. No discontinuity should be observed in the O-C magnitude variation or in its slope as the system 
goes into primary eclipse; the smooth variation throughout primary eclipse in Fig. 4.1.5(c) shows good agreement between the observed eclipse characteristics and the WINK model. Figs. 4.1 .5 (a), (b) and (c) illustrate the changes in the spot wave between 1979 August and 1980 september. A slow migration towards decreasing orbital phase is apparent, as is a gradual decrease in the depth of wave minimum. All three lightcurves have rather poor coverage near wave maximum; however, the brightness of the system near wave maximum appears to be decreasing. A likely explanation is that an initially compact group of starspots, subjected to shearing due to the differential rotation producing the wave migration, has become more widely dispersed in longitude throughout the period of observation, without any significant decrease in the total spot area. Dr. K. Thompson has communicated privately that his own photoelectric observations of HD 5303 made between 1980 september 29 and 1981 February 3 U.T. show a spot wave with the same phase of wave minimum, but a total amplitude of only 0.06 magnitude. This suggests that the group had been almost completely dispersed by this time.

The orbital inclination of $67^{\circ}$ obtained from the photometric model gives a total mass of $2.32 \pm 0.02 \mathrm{M}_{\odot}$ for the system. With $q \simeq 1$, the masses for both components are close to $1.16 \mathrm{M}_{\odot}$ for both components, and agree well with the $F 7 / 8 \mathrm{~V}$ spectral type obtained for the hotter star. 
TABLE 4.1 .2

(a) Adopted photometric parameters for HD 5303 WINK lightcurve synthesis.

$$
\begin{array}{ll}
\mathrm{P} & =2.79786 \text { days } \\
\mathrm{T}_{\text {conj }} & =\text { HJD } 2444499.040 \\
\mathrm{i} & =67^{\circ} .0 \pm 0^{\circ} .5 \\
\mathrm{e} & =0.00 \text { (assumed) } \\
\mathrm{k} & =0.42 \pm 0.01 \\
\frac{\mathrm{r}_{\mathrm{h}}}{\mathrm{a}} & =0.146 \pm 0.005 \\
\frac{\mathrm{r}_{\mathrm{c}}}{\mathrm{a}} & =0.350 \pm 0.020
\end{array}
$$

\begin{tabular}{|c|c|c|c|c|}
\hline star & & & hot & cool \\
\hline Surface gravity & $\log g$ & $=$ & 4.0 & 3.5 \\
\hline Limb darkening & $u_{b}$ & $=$ & 0.8 & 0.9 \\
\hline & $u_{v}$ & $=$ & 0.6 & 0.6 \\
\hline & $u_{r}$ & $=$ & 0.5 & 0.6 \\
\hline & $u_{i}$ & $=$ & 0.5 & 0.5 \\
\hline $\begin{array}{l}\text { Gravity } \\
\quad \text { brightening }\end{array}$ & $\beta$ & $=$ & 0.08 & 0.08 \\
\hline Albedo & $w$ & $=$ & 1.0 & 0.5 \\
\hline $\begin{array}{l}\text { Effective } \\
\text { temperature }\end{array}$ & $\mathrm{T}_{\mathrm{e}}$ & $=$ & $6110 \mathrm{~K}$ & 4500 \\
\hline
\end{tabular}


(b) Physical parameters calculated by WINK.

\begin{tabular}{|c|c|c|c|c|c|}
\hline star & & \multicolumn{2}{|l|}{ hot } & \multicolumn{2}{|l|}{ cool } \\
\hline $\mathrm{T}$ (equator) & & 6110 & K & 4500 & K \\
\hline $\mathrm{T}$ (pole) & & 6116 & $\mathrm{~K}$ & 4556 & K \\
\hline Semi-axes A & & 0.147 & & 0.381 & \\
\hline $\mathrm{B}$ & & 0.146 & & 0.358 & \\
\hline $\mathrm{C}$ & & 0.145 & & 0.343 & \\
\hline Normalised & $I_{b}$ & $=0.74(0.72 \pm$ & $.02)$ & $0.2610 .28 \mp$ & $.02)$ \\
\hline Iuminosities & $1_{\mathrm{v}}$ & $=0.58(0.60 \pm$ & .021 & $0.4210 .40 \mp$ & .021 \\
\hline $\begin{array}{l}\text { Cobserved } \\
\text { values in }\end{array}$ & $I_{r}$ & $=0.51(0.50 \pm$ & .021 & $0.4910 .50 \mp$ & $.02)$ \\
\hline parentheses) & $1_{i}$ & $=0.42(0.41 \pm$ & $.02)$ & $0.5810 .59 \mp$ & $.02)$ \\
\hline
\end{tabular}



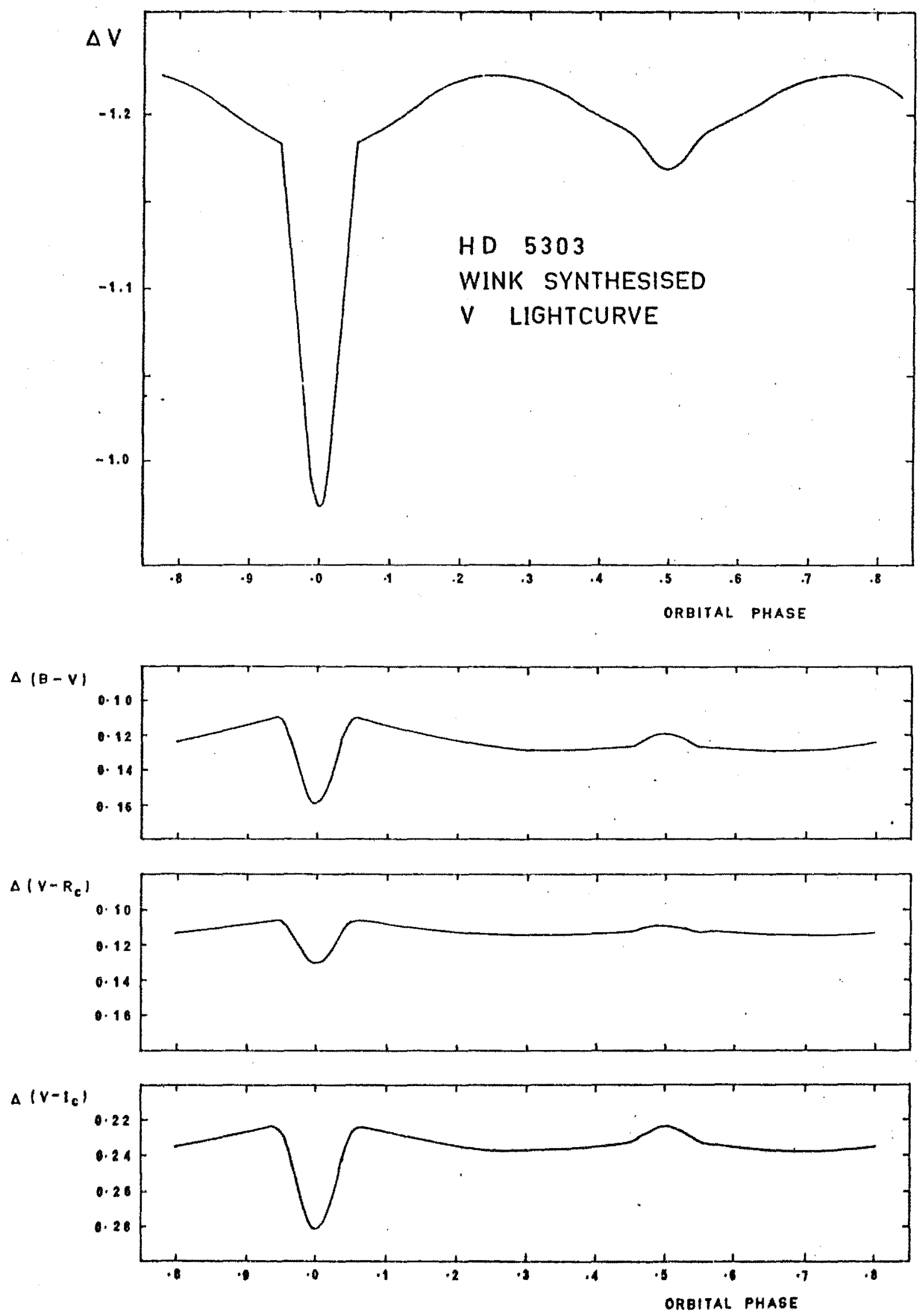

Fig. 4.1.4 WINK synthesised lightcurves for HD 5303 in $V, B-V, \quad V-R_{C}, \quad V-I_{C}$. Comparison star is HD 5210. 

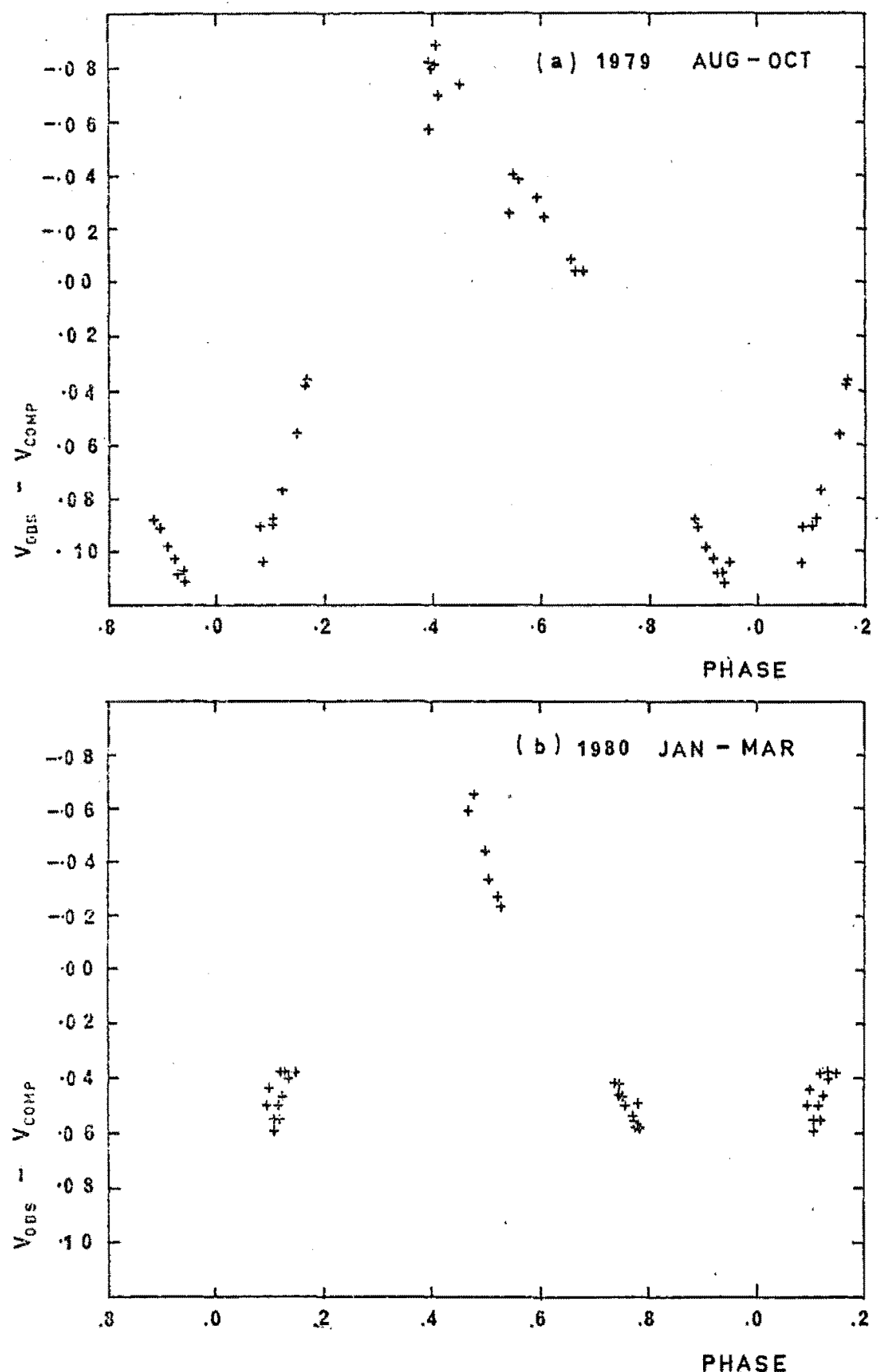

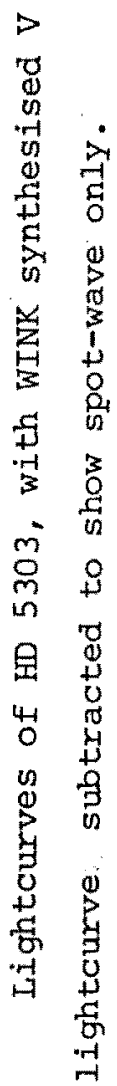

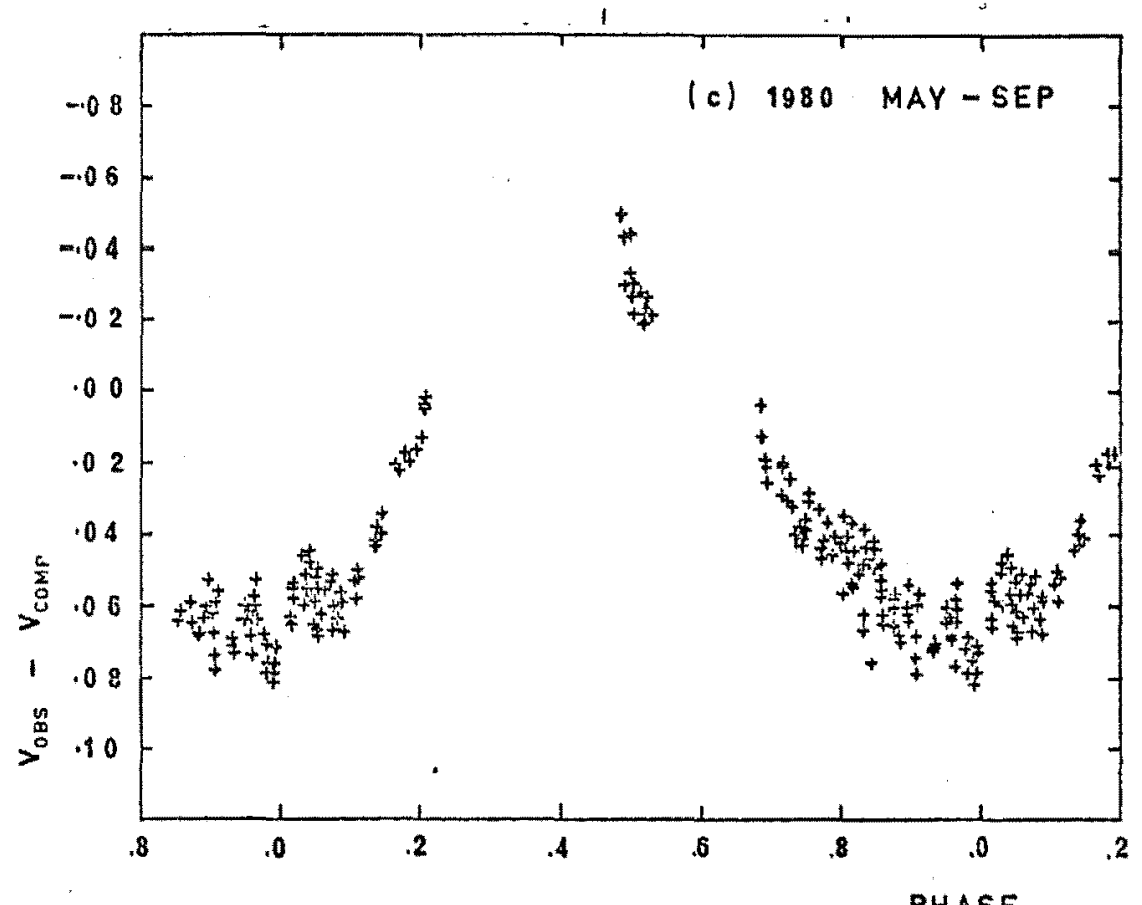

ó

+1 
4.2 HD 8435

The presence of strong Ca II emission in the spectrum of HD 8435 was noted in the objective prism surveys of Bidelman and MacConnell (1973) and Houk and Cowley (1975). The presence of possible Balmer emission is noted in the latter survey.

\subsubsection{Radial Velocity Observations}

Fifteen coudé spectra of the Mg I b line region in HD 8435 were obtained at MSO during 1980 October and November.

Cross correlation of the spectra with the template spectrum of HR 141 revealed that HD 8435 is a short-period double-lined spectroscopic binary. The c.c.f.s show significant changes in shape over periods of a few hours, as illustrated in Fig. 4.2.1. At no time is the separation of the c.c.f. peaks sufficient to permit accurate measurement of the two components' individual velocities.

However, four conjunctions of the system were observed, for which it was possible to determine which star was in front. This involved selecting the narrowest and most symmetrical of the ccf profiles obtained on nights when the direction of motion of the fainter spectrum could be reliably determined. For instance, if the fainter spectrum showed an increasing redshift with respect to the brighter spectrum before and after conjunction, the fainter star was in front at conjunction and vice versa. The four conjunctions observed in this. way were at

$\begin{array}{lll}\text { HJD } & 2444538.05 \pm 0.01 & \text { (brighter star in front) } \\ 2444539.03 \pm 0.01 & \text { (fainter " " } " \text { (fainter } \\ 2444565.98 \pm 0.02 & \text { (brighter " } \\ 2444566.98 \pm 0.01 & \text { " ) }\end{array}$


Inspection of the data suggests that the most probable period for the system is $P=0.6576 \pm 0.0005$ days.

The measured velocities of the stronger spectrum in HD 8435, uncorrected for the effects of blending with the secondary spectrum, are listed in Table 4.2.1.

Using these velocities, a plot was constructed of the brighter component's heliocentric radial velocity against the sine of the orbital phase, measured from the time of conjunction with the brighter star in front $\left(T_{0}=2444538.05 \pm .01\right)$. This plot (Fig. 4.2.2) shows considerable scatter owing to blending effects with the secondary spectrum. However, a definite correlation is present, and this indicates that the adopted orbital period is correct.

A linear least-squares fit to the data in Fig.4.2.2 gives

$$
\begin{aligned}
& \gamma=7 \pm 2 \quad \mathrm{~km} \mathrm{~s}^{-1} \\
& \mathrm{~K}_{\mathrm{A}}=38 \pm 5 \mathrm{~km} \mathrm{~s}
\end{aligned}
$$

where component $A$ is defined to be the star showing the stronger spectrum at this wavelength.

In only two of the spectra is the velocity separation of the two stars sufficient for an estimate of the secondary's velocity to be made. These two values (Table 4.2 .1 ) suggest that $K_{B} \simeq 50 \pm 10 \mathrm{~km} \mathrm{~s}^{-1}$, giving a mass ratio $q=0.8 \pm 0.2$ and a system mass $\left(M_{A}+M_{B}\right) \sin ^{3} i=(5 \pm 2)$ $\times 10^{-2} M_{\odot}$. 
TABLE 4.2 .1

\begin{tabular}{|c|c|c|c|c|c|}
\hline \multicolumn{2}{|c|}{$\begin{array}{l}\text { Date } \\
\text { U.T. }\end{array}$} & $\begin{array}{l}\text { Exposure } \\
\text { (sec.) }\end{array}$ & $\begin{array}{l}\text { HJD } \\
2444000+\end{array}$ & $\begin{array}{c}\text { Heliocentric radial } \\
\mathrm{A}\end{array}$ & $\begin{array}{c}\text { velocity } \\
B\end{array}$ \\
\hline \multirow[t]{2}{*}{1980} & oct. 25 & 300 & 537.973 & -11.7 & \\
\hline & & 400 & 538.046 & 6.8 & \\
\hline \multirow[t]{4}{*}{1980} & Oct. 26 & 300 & 538.944 & 22.4 & \\
\hline & & 550 & 539.026 & 7.8 & \\
\hline & & 300 & 539.127 & -18.5 & \\
\hline & & 400 & 539.172 & -34.6 & $60 ?$ \\
\hline \multirow[t]{2}{*}{1980} & Nov. 22 & 300 & 565.979 & -4.9 & \\
\hline & & 400 & 566.024 & -4.8 & \\
\hline \multirow[t]{6}{*}{1980} & Nov. 23 & 300 & 566.967 & 7.2 & \\
\hline & & $"$ & 566.987 & 1.8 & \\
\hline & & $"$ & 567.028 & 39.5 & \\
\hline & & 400 & 567.074 & 42.0 & \\
\hline & & $"$ & 567.125 & 57.5 & $-50 ?$ \\
\hline & & $"$ & 567.176 & 35.3 & \\
\hline 1980 & Nov .24 & $"$ & 568.006 & -6.0 & \\
\hline
\end{tabular}

Table 4.2.1 Radial velocities of HD 8435 from Mg I b spectra, MSO 1980. 

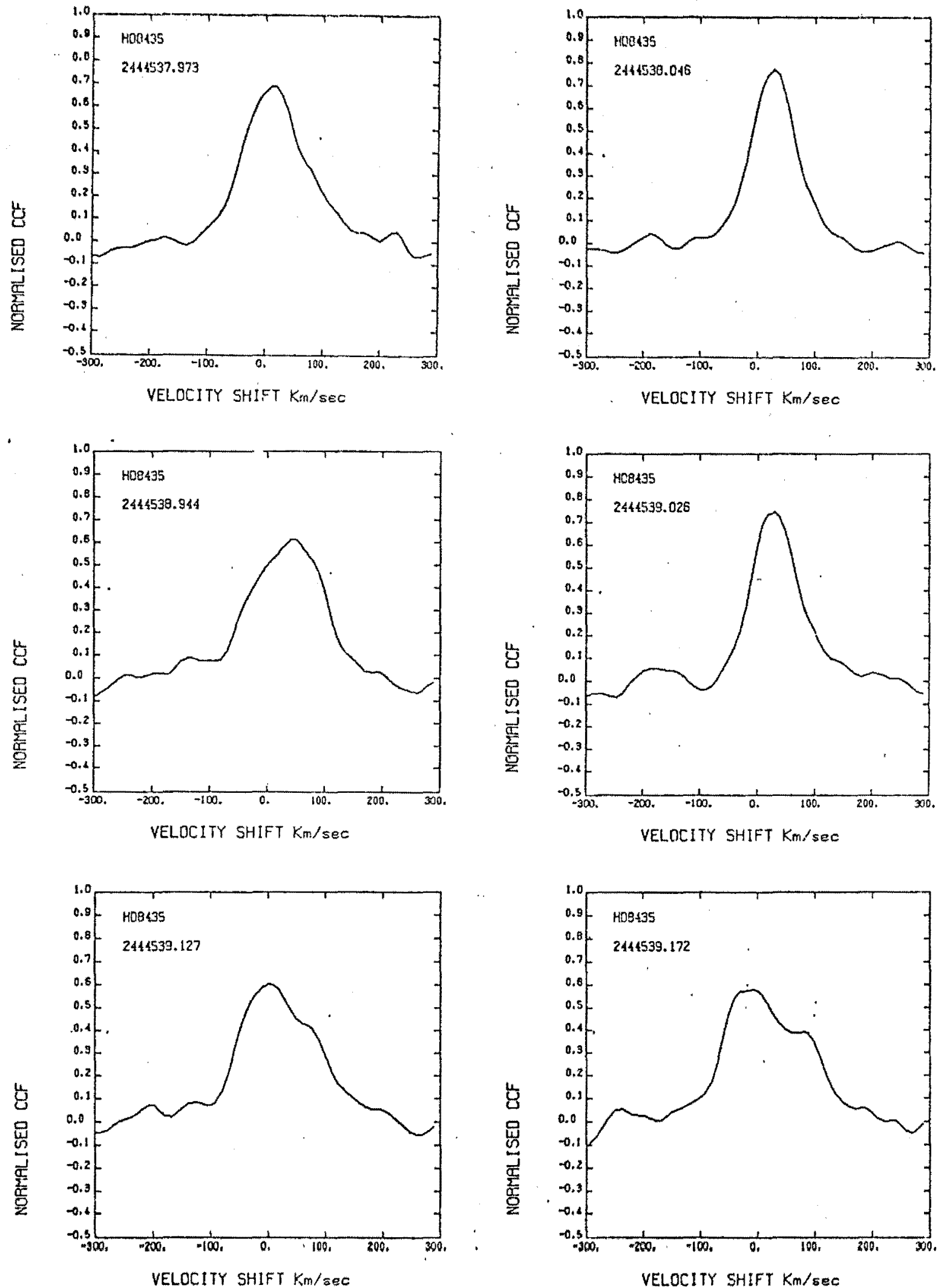

Fig. 4.2.1 Cross-correlations of HD $8435 \mathrm{Mg}$ I b spectra with HR 141 template, 1980 October and November. Velocity scale as for Fig. 4.1.1. 

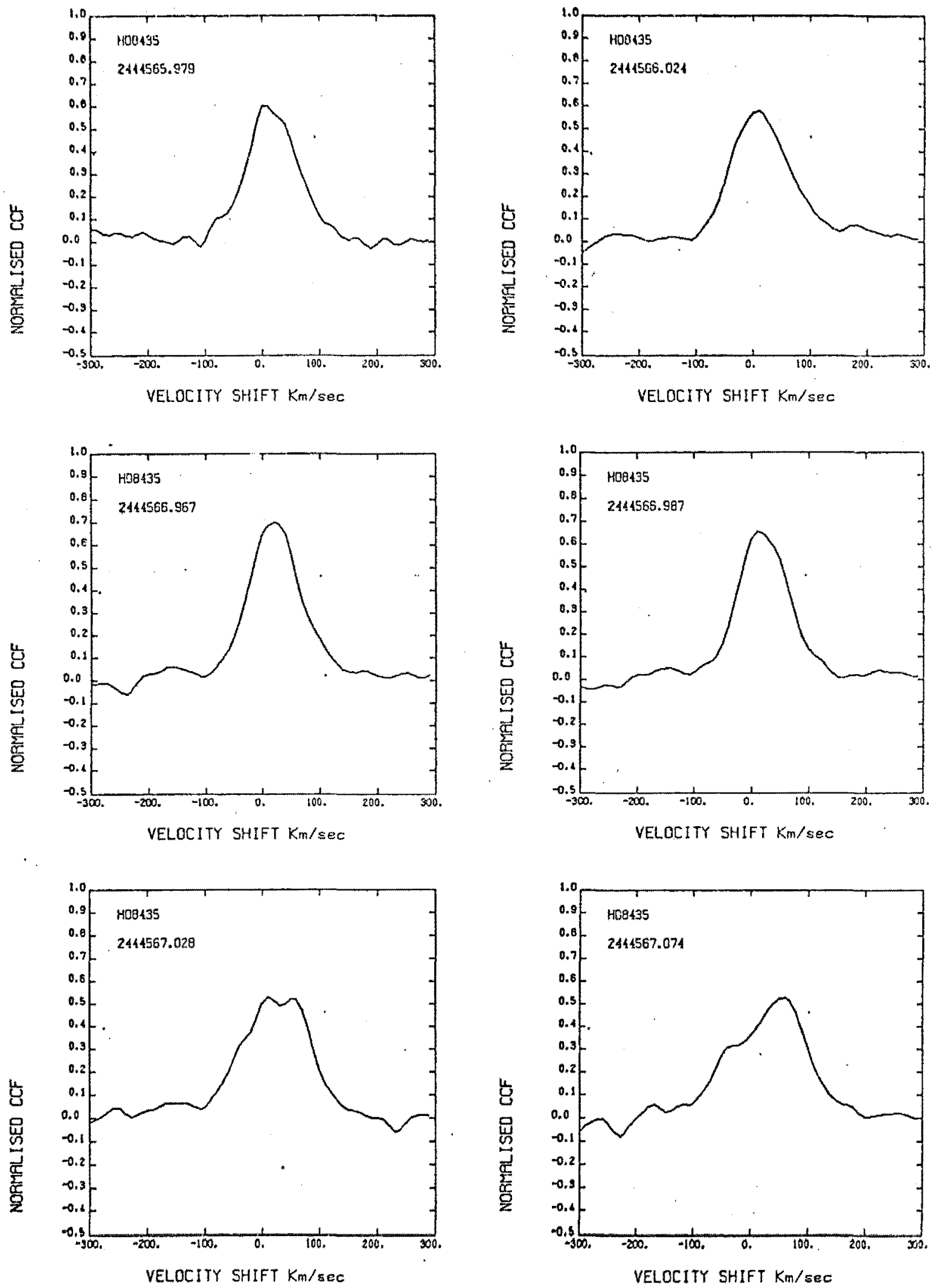

Fig. 4.2.1 (Continued) 

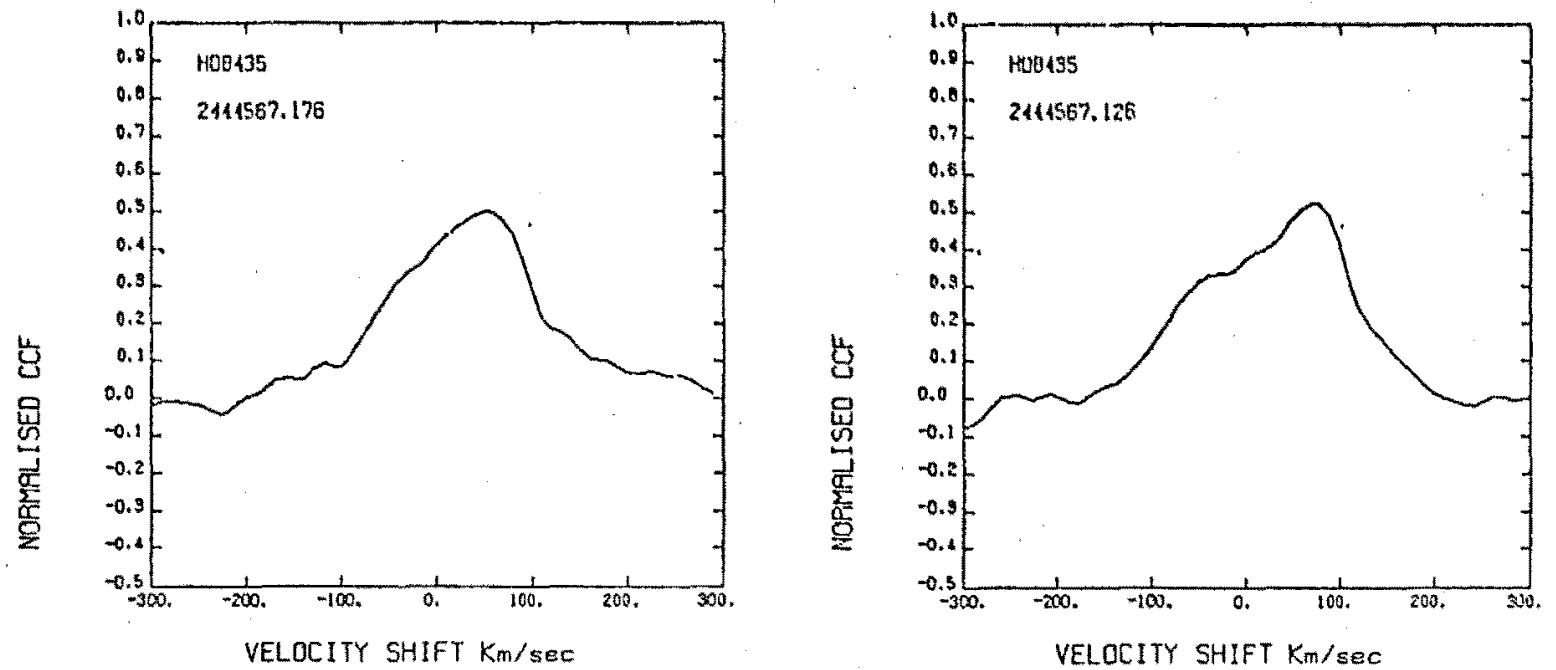

Fig. 4.2.1 (Continued)

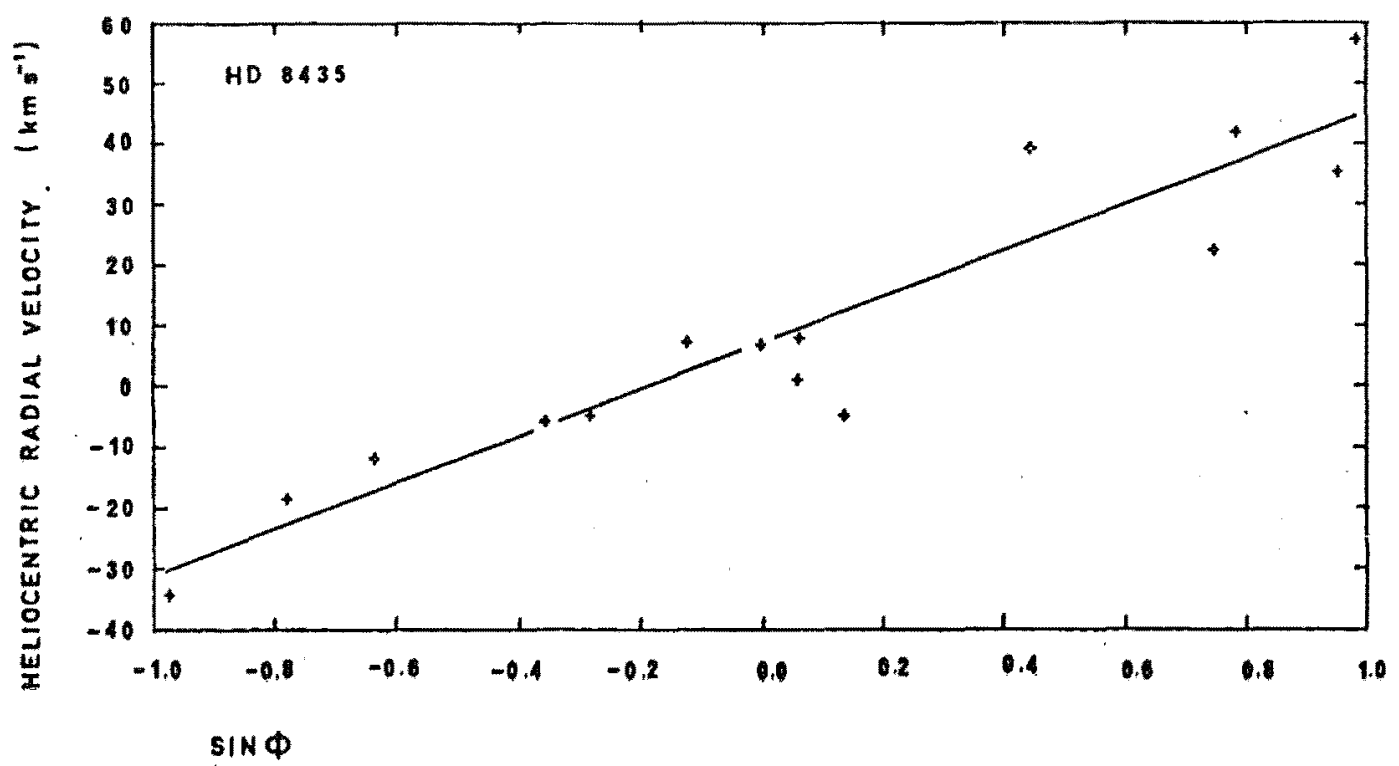

Fig. 4.2.2 Heliocentric radial velocity of stronger c.c.f. peak vs. $\sin \phi$ for HD 8435 . The ephemeris used was HJD $=2444538.05+0.6575 \mathrm{E}$. 
NORIRLISED INTENSITY

$\widehat{ล}$

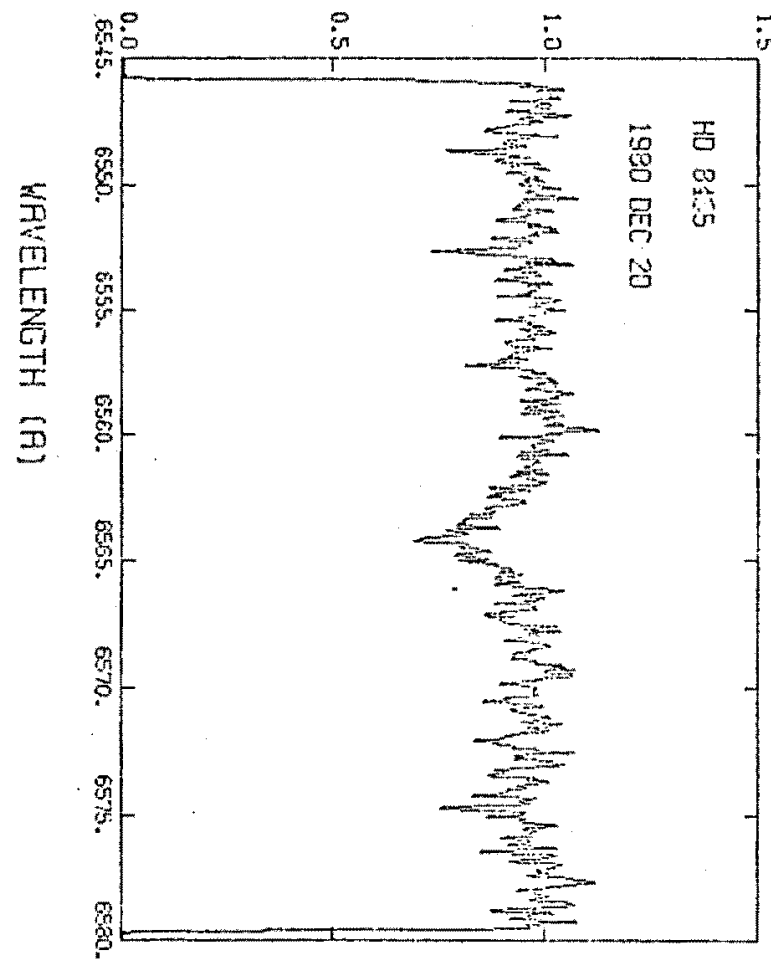

NORMRLISEO INTENSITY

Q

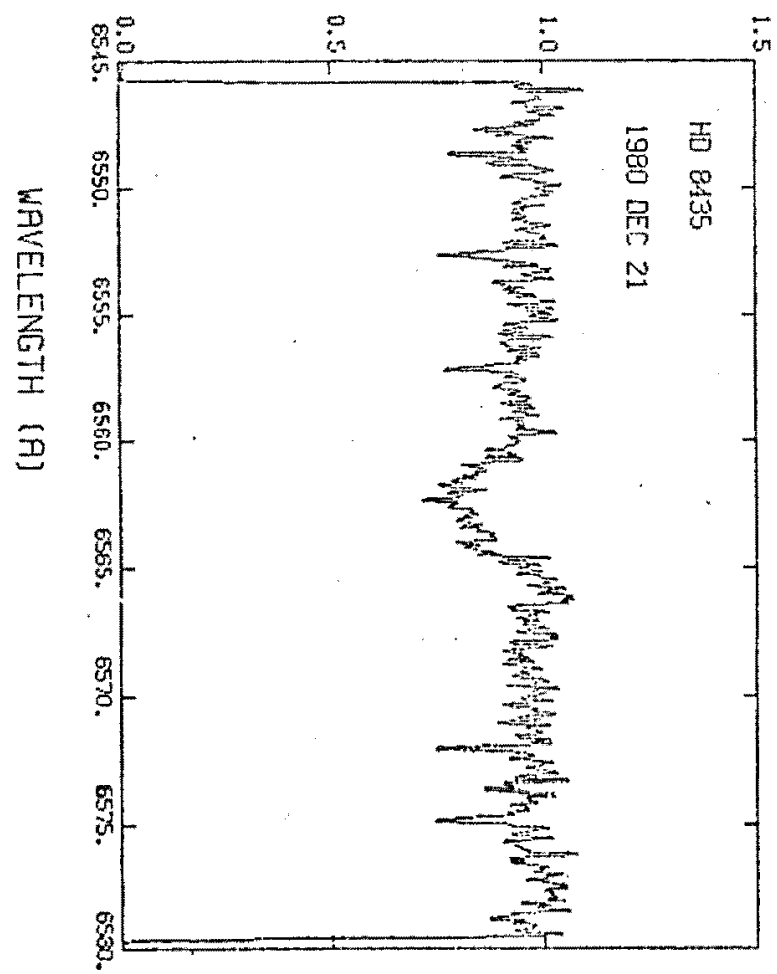

NORMALISED INTENSITY

宛

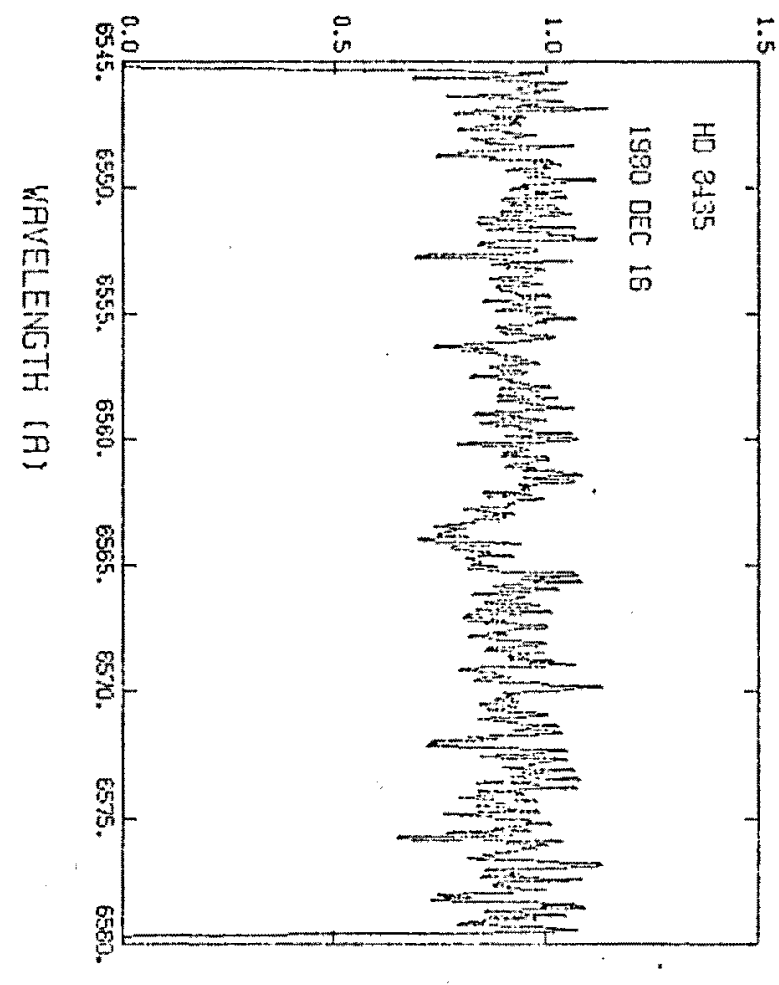

NORMAL ISEO INTENSITY

Ðே

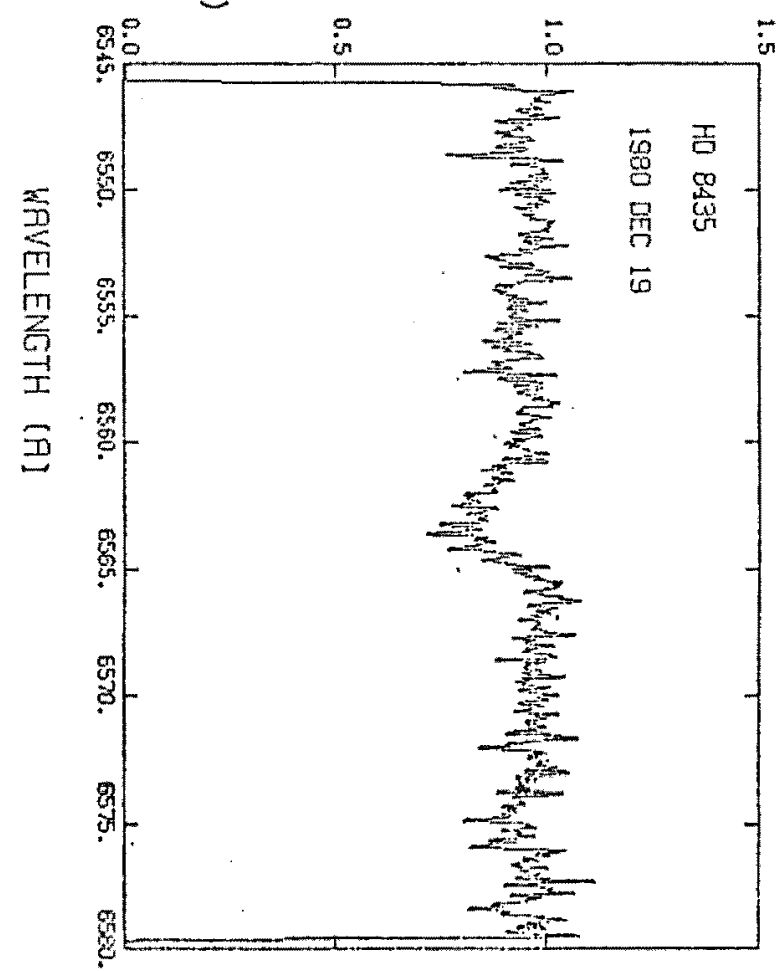

Fig. 4.2 .3 Ho spectra of HD 8435,1980 December. Orbital phases
are
(a) $\phi=0.056$,
(b) $\phi=0.531$,
(c) 0.234 ,
(d) 0.588 . 


\section{$4.2 .2 \quad H \alpha$ Spectra}

Four $\mathrm{H}_{\alpha}$ spectra of HD 8435 were obtained at MSO, on consecutive nights during 1980 December (Fig. 4.2.3). In all four spectra, $\mathrm{H} \alpha$ appears as a broad, shallow absorption feature; none of the nearby weak metallic lines are identjfiable, owing to high rotational smearing. The shallowness of the $\mathrm{H} \alpha$ absorption indicates that continuous low-level flare activity is present in one or both components.

\subsubsection{Photometric Observations}

Photometry of HD 8435 in the Cousins BVR ${ }_{\mathrm{C}}{ }_{\mathrm{C}}$ system was obtained at MSO on four nights during 1980 November and December, and at MJUO on four nights during 1981 october. No accurate determination of colour indices was made because of unstable weather conditions. However, approximate values were obtained at MJUO.

$$
\begin{aligned}
& (B-V)=0.7 \pm 0.1 \\
& \left(V-R_{C}\right)=0.4 \pm 0.1 \\
& \left(V-I_{C}\right)=0.8 \pm 0.1
\end{aligned}
$$

These are in agree ment with the G6/8 combined spectraI type given by Houk and Cowley (1975), although their Iuminosity class of III/IV is not consistent with the short binary period.

The comparison star was HD 7679 for both the MSO and MJUO observations. Observations of a third check star were made regularly on all nights, and no significant variability was detected in the comparison star.

HD 8435 itself was found to be variable, with an amplitude in $\mathrm{V}$ of 0.05 magnitude. The $\mathrm{V}$ observations made at MSO during 1980 November and December are plotted against orbital phase in Fig. 4.2.4. The lightcurve appears to have two minima, the deeper of which appears near phase 0.5 and 
the shallower near phase 0.0 . It will be recalled that orbital phase 0.0 was defined as being conjunction with the star giving rise to the stronger absorption spectrum at the Mg I b lines in front. If this star is the hotter of the two, the shape of the lightcurve would be consistent with a variation due to tidal distortion of the stars and the reflection effect alone. No definite evidence for either eclipses or a spotwave is seen.

The MJUO observations show a similar variation, but the uncertainty in the orbital period was such that the orbital phase of photometric minimum could not be established.

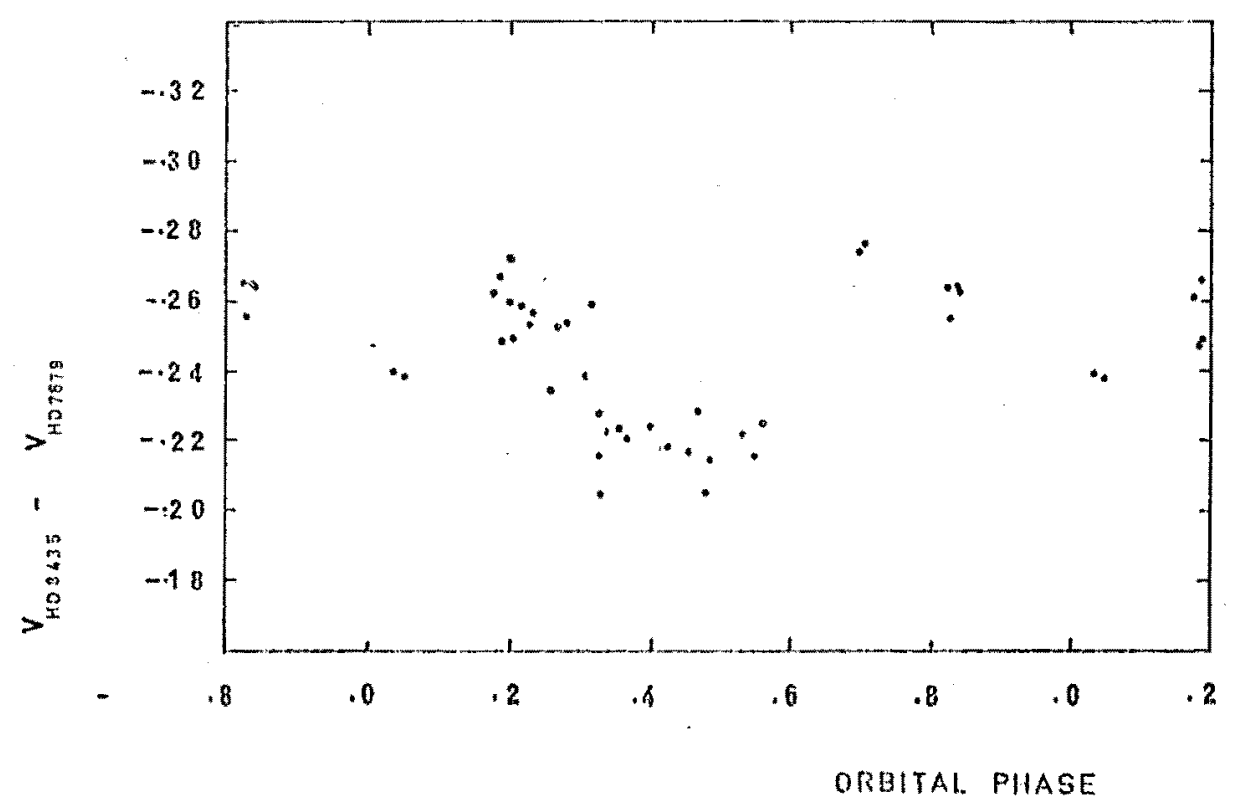

Fig. 4.2.4 V lightcurve of HD 8435, 1980 November and December. 
4.2 .4 Summary

HD 8435 is a probable new member of the short-period ( $\mathrm{P}<1$ day) group of RS CVn-like binaries (Hal1, 1976). Further photometric and spectroscopic observations should be made in order to ascertain the individual spectral types of the components, and whether or not the system is detached. High resolution spectra of the $\mathrm{Ca}$ II $\mathrm{H}$ and $\mathrm{K}$ lines would be of interest, as the existing observations do not permit a conclusive identification of the active component to be made. Indeed, the relative intensities of the Mg I b spectra and the shallowness of the $\mathrm{H} \alpha$ profile, suggests that both components may be active.

The hotter component is probably a mid-G dwarf; if a system mass of $\sim 1.7 \mathrm{M}_{\odot}$ is assumed, the orbital inclination of the system is about $20^{\circ}$. Such a mass would also imply an orbital separation of about $3.8 \mathrm{R}_{\odot}$, making it unlikely that the system is a W UMa contact binary. 
4.3 HD 14643

HD 14643 has been noted for its $\mathrm{Ca}$. II $\mathrm{H}$ and $\mathrm{K}$ emission by Bidelman and MacConnell (1973) and Houk and Cowley (1975). Houk and Cowley note that the spectrum appears to be composite; $\mathrm{HD} 14643$ is also a visual double (Innes 458 ) with $\mathrm{p}=247^{\circ}$ and separation $0.6^{\prime \prime}$, with magnitudes 8.3 and 10.4 .

\subsubsection{Radial Velocity observations}

Ten spectra of the Mg I b line region in HD 14643 were obtained at MSO during 1980 October and November. The single absorption spectrum showed radial velocity variations with a total amplitude of at least $60 \mathrm{~km} \mathrm{~s}^{-1}$. The radial velocity data are summarised in Table 4.3 .1 .

\subsubsection{Ha spectra}

Three $\mathrm{H}_{\alpha}$ spectra were obtained with the coudé échelle spectrograph on the MSO $1.88 \mathrm{~m}$ telescope during 1980 December. All three spectra showed $\mathrm{H} \alpha$ as an absorption feature, somewhat broadened by rotation but otherwise apparently normal. The metallic absorption lines near $\mathrm{H} \alpha$ exhibit rotationally-smeared profiles corresponding to $v_{e} \sin i=$ $25 \pm 5 \mathrm{~km} \mathrm{~s}^{-1}$. The three $\mathrm{H} \alpha$ spectra are reproduced in Fig. 4.3.1, and the radial velocities obtained from the metallic lines are listed in Table 4.3.1. A period analysis of the radial velocity data using the method of Gieseking (1981) produced a periodogram with heavy aliasing (Fig.4.3.2) due to undersampling. However, inspection of the raw velocities suggests that the three most likely periods are $7.94,11.13$ and 18.73 days, with corresponding velocity amplitudes $29.1,27.6$ and $29.1 \mathrm{~km} \mathrm{~s}^{-1}$ respectively. 

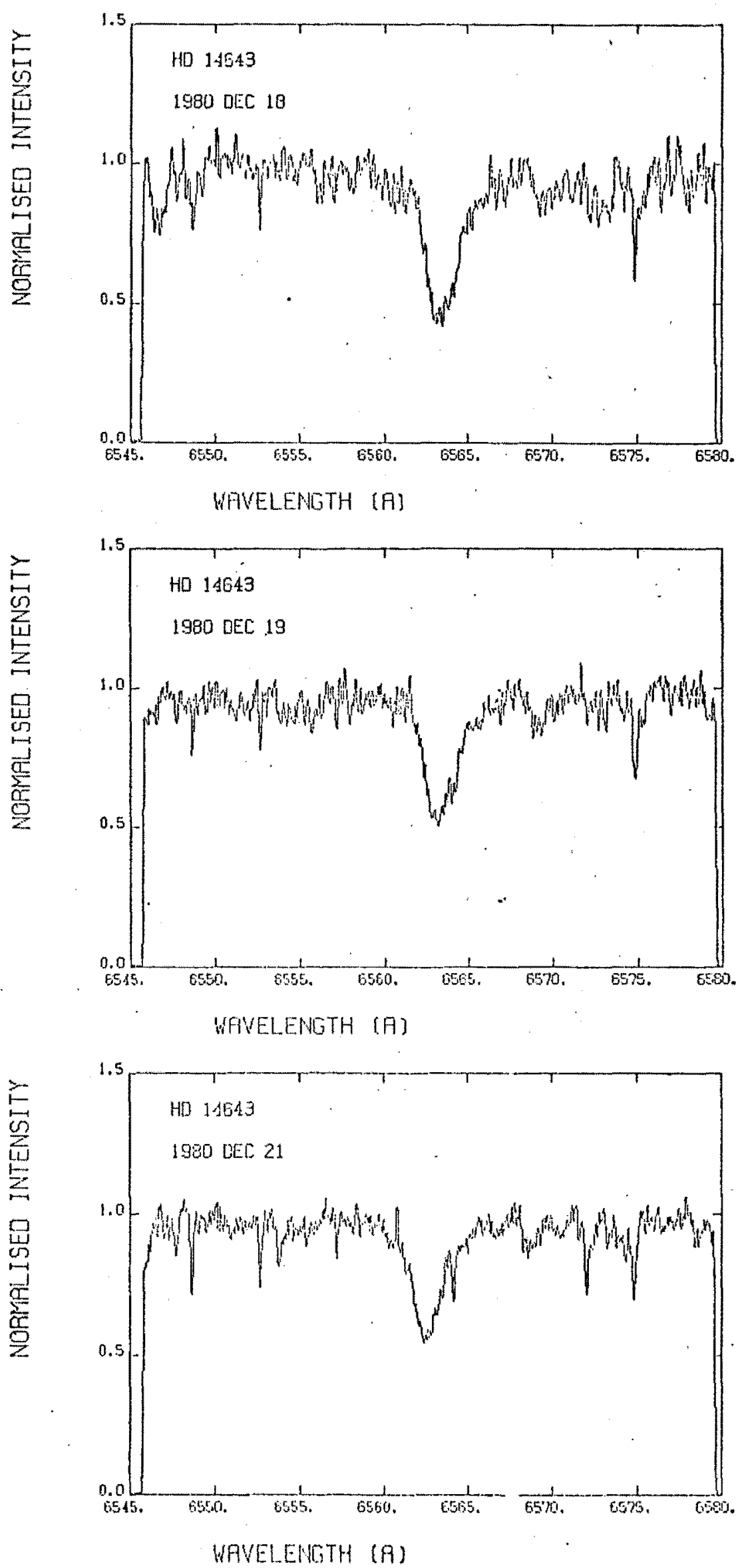

Fig. 4.3.1 H $\alpha$ spectra of HD 14643. 
a

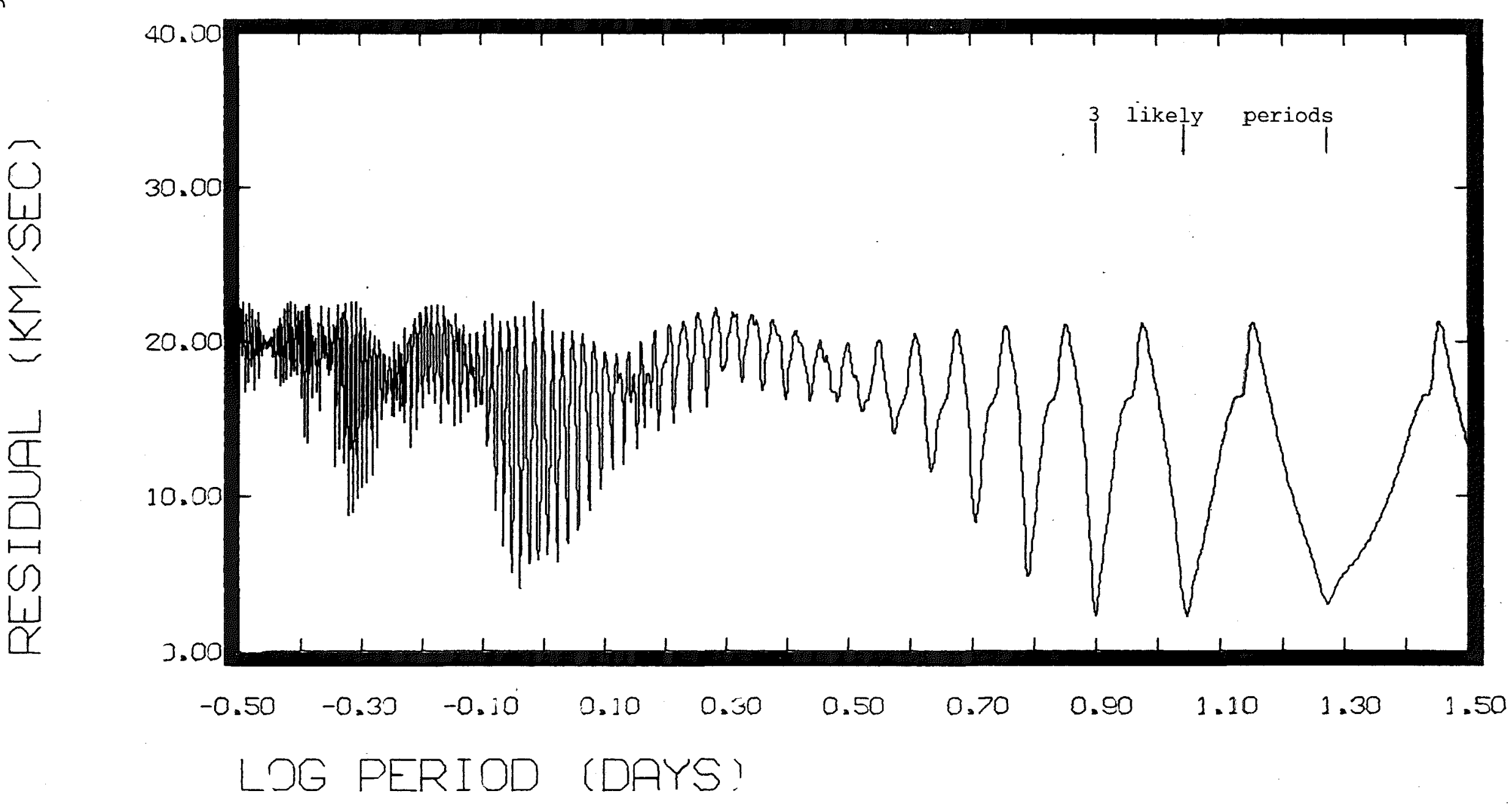

Fig. 4.3.2 Periodogram for $\mathrm{HD} 14643$ radial velocities. 
TABLE 4.3 .1

\begin{tabular}{|c|c|c|c|c|c|}
\hline \multicolumn{2}{|c|}{$\begin{array}{l}\text { Date } \\
\text { U.T. }\end{array}$} & \multirow{2}{*}{$\begin{array}{c}\text { Exposure } \\
\text { (sec.) } \\
400\end{array}$} & \multirow{2}{*}{$\begin{array}{c}\text { HJD } \\
2444000+ \\
538.057\end{array}$} & 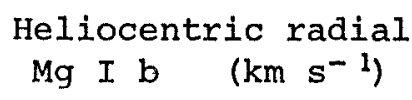 & \multirow{2}{*}{$\begin{array}{l}\text { velocity } \\
\qquad \begin{array}{l}\mathrm{H} \alpha \\
\end{array}\end{array}$} \\
\hline 1980 & Oct. 25 & & & -8.2 & \\
\hline & $"$ & 300 & 538.154 & -11.1 & \\
\hline 1980 & Oct. 26 & 300 & 538.963 & -19.2 & \\
\hline 1980 & Nov. 22 & $"$ & 565.986 & 24.0 & \\
\hline & $"$ & $"$ & 566.029 & 25.7 & \\
\hline 1980 & Nov. 23 & $"$ & 566.973 & 36.6 & \\
\hline & $"$ & $"$ & 566.993 & 35.7 & \\
\hline & $"$ & $"$ & 567.034 & 39.4 & \\
\hline & $"$ & $"$ & 567.080 & 37.0 & \\
\hline & $"$ & $"$ & 567.135 & 37.0 & \\
\hline 1980 & Dec. 18 & 1800 & 593.10 & & -1.4 \\
\hline 1980 & Dec. 19 & 2050 & 594.01 & & -10.1 \\
\hline 1980 & Dec. 21 & 3600 & 596.04 & & -12.1 \\
\hline
\end{tabular}

Table 4.3.1 Radial velocities of HD 14643 from Mg I b and Ha spectra, MSO, 1980.

4.3 .3 Photometric Observations

Differential photometry in the Cousins $B V R_{C} I_{C}$ system made at MJUO during 1979 October and at MSO during 1980 November and December revealed variability with an amplitude of at least $0.15 \mathrm{mag}$. in HD 14643. However, insufficient data were obtained for a photometric period analysis to be performed. The colour indices of the system (Table 2-3) do not fit the mean relations of Johnson (1966). The unusually flat energy distribution would be consistent with HD 14643 being a composite spectrum object, as suggested by Houk and Cowley (1975). High-resolution blue spectra of this 
object would be of interest; if HD 14643 consists of an active G-K subgiant and a hotter main sequence companion, the hotter component will probably contribute significantly to the system's luminosity at the blue end of the spectrum, whereas at red wavelengths the binary is single-lined (cf. HD 101309).

\section{$4.4 \quad$ HD 32918}

The presence of $\mathrm{Ca}$ II $\mathrm{H}$ and $\mathrm{K}$ emission in the spectrum of HD 32918 is noted by both Bidelman and Macconnell (1973) and Houk and Cowley (1975). Houk and Cowley classify the spectrum as KIIIIp.

The results of the photometric and spectroscopic observations of HD 32918 made during this project have been published by Collier (1982). However, further analysis of the photometric data is presented here.

\subsubsection{Colour changes and Spot temperatures in HD 32918}

HD 32918 exhibits a rotationally-modulated light variation with a period of 9.55 days and an amplitude of 0.3 magnitude in $V$. The light variation is accompanied by a colour change, with the star appearing bluest near wave maximum.

As little variation may be seen during the course of a single night, mean values of the differential colour indices $\Delta(B-V), \Delta\left(V-R_{C}\right)$ and $\Delta\left(V-I_{C}\right)$ were evaluated for each of the twelve nights on which HD 32918 was observed from MJUO. These mean values are plotted against $\Delta V$ in Fig. 4.4.1; the error bars represent the standard deviations for the sample means, being the standard deviation for a single observation divided by the square root of the number of observations made on the night concerned. The observations were reduced to apparent luminosities 

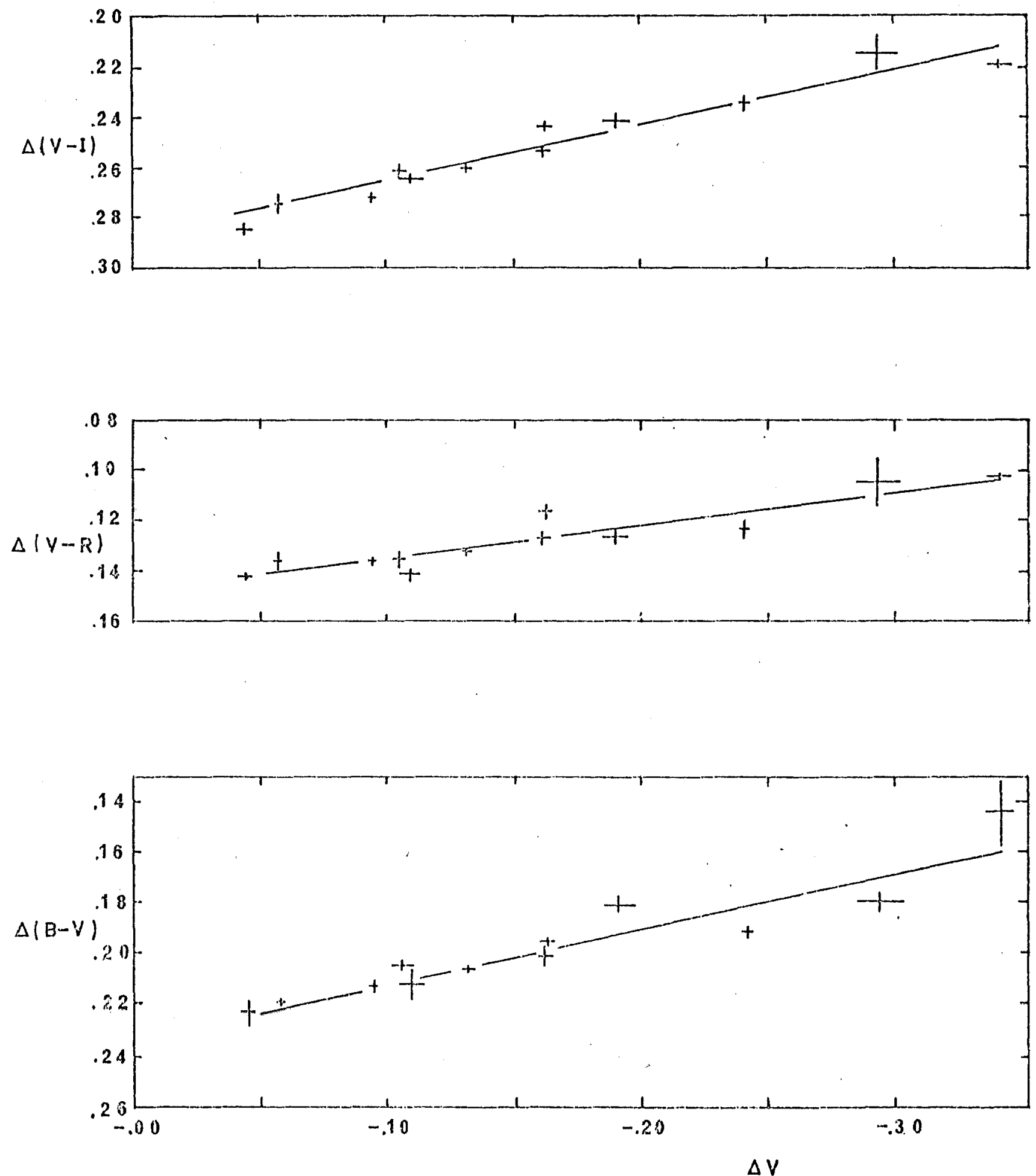

Fig. 4.4.1 Variations of colour indices with $V$ magnitude in HD 32918. 
in the cousins $B, V, R_{C}$ and $I_{C}$ bands, taking as unit luminosity the observed luminosity in the $\mathrm{V}$ band at wave maximum

$$
\begin{aligned}
& 1_{\max }^{\mathrm{V}} \equiv 1 \quad(4.4-1(\mathrm{a})) \\
& 1^{\mathrm{V}} \quad=10^{-0.4}\left(\mathrm{~V}-\mathrm{V}_{\text {max }}\right)(4.4-1(\mathrm{~b})) \\
& 1^{\mathrm{B}}=1^{\mathrm{V}} \cdot 10^{-0.4}(\mathrm{~B}-\mathrm{V})(4.4-1 \text { (C)) } \\
& I^{R_{C}}=I^{V} \cdot 10^{+0.4}\left(V-R_{C}\right)(4.4-I(d)) \\
& I^{I_{C}}=I^{V} \cdot 10^{+0.4}\left(V-I_{C}\right)(4.4-1(\mathrm{e}))
\end{aligned}
$$

Linear least-squares fits to the resulting fluxes gave the relations

$$
\begin{aligned}
I^{B} & =0.452 I^{V}-0.072 \quad(4.4-2(\mathrm{a})) \\
I_{C}^{R_{C}} & =1.520 I^{V}+0.207 \quad(4.4-2(\mathrm{~b})) \\
I^{I} \mathrm{C} & =2.365 I^{V}+0.604 \quad(4.4-2(\mathrm{c})) \\
& \text { for } 0.761 \leqslant I^{V} \leqslant 1.000
\end{aligned}
$$

The solid curves in Fig. 4.4.1 represent these three relations $(4.4-2)$ converted back to differential magnitudes. The deviations of the observed colours from the fitted relations compare satisfactorily with the observational uncertainties.

The differential $\mathrm{V}$ magnitudes are plotted modulo rotational phase in Fig. 1 of Collier (1982). As may be seen from the lightcurve, the phase interval around wave maximum is rather poorly covered. However, the observations presented here represent adequately the range of variation observed in HD 32918 between 1979 August and 1980 February. In order to estimate mean effective temperatures for the "hot" and "cool" hemispheres of HD 32918, we must first apply corrections to the observed colour indices to allow for the effects of interstellar reddening. The absolute 
magnitude of $\mathrm{HD} 32918$ is $\mathrm{M}_{\mathrm{v}}=1.0 \pm 1.0$ (Collier, 1982), giving a distance modulus of $8.0 \pm 1.0 \mathrm{mag}$. This figure is similar to that obtained by Sanduleak and Philip (1968) for an association of early main sequence stars seen as foreground objects superimposed on the LMC at $\alpha_{1950}=5^{\mathrm{h}} 18^{\mathrm{m}}$, $\delta=-68^{\circ} .5$. The mean interstellar reddening for this association is $\mathrm{E}(\mathrm{B}-\mathrm{V})=0.065$. HD 32918, at $\alpha_{1950}=5^{\mathrm{h}} 00^{\mathrm{m}}$, $\delta_{1950}=-75^{\circ}$, lies a similar distance below the galactic plane in the same direction, and so we shall adopt the same reddening value obtained for the association.

The theoretical extinction curve No. 15 of van de Hulst, given in Johnson (1966 a) was used to estimate the $\left(V-R_{J}\right)$ 。 and $\left(\mathrm{V}-\mathrm{I}_{\mathrm{J}}\right)_{\mathrm{O}}$ colour indices of HD 32918 at wave maximum and wave minimum.

The van de Hulst curve gives

$$
\begin{array}{ll}
\frac{E\left(V-R_{J}\right)}{E(B-V)}=0.80 & (4.4-3(a)) \\
\frac{E\left(V-I_{J}\right)}{E(B-V)}=1.62 & (4.4-3(b))
\end{array}
$$

Taking $E(B-V)=0.07$, the unreddened. $\left(V-R_{J}\right)$ colour indices at wave maximum and wave minimum are

$$
\begin{aligned}
& (\mathrm{B}-\mathrm{V}){\underset{\mathrm{m}}{\min }}_{\max }^{\max }=0.99 \quad\left(\mathrm{~V}-\mathrm{R}_{\mathrm{J}}\right)_{\mathrm{O}}^{\max }=0.80 \quad\left(\mathrm{~V}-\mathrm{I}_{\mathrm{J}}\right)_{\mathrm{O}}^{\max }=1.35 \\
& \left.(B-V)\right|_{0} ^{\min }=1.06 \quad\left(V-R_{J}\right)_{0}^{\min }=0.86 \quad\left(V-I_{J}\right)_{0}^{\mathrm{min}}=1.43
\end{aligned}
$$

The mean relations of Johnson (1966) for luminosity class IV/V give mean effective temperatures of 4940 and 4700 $K$ for the hemispheres facing the observer at wave maximum and minimum respectively. If, as seems likely, the brighter hemisphere is not completely free of spot regions, the resulting under-estimation of the photospheric temperature will probably amount to no more than $200 \mathrm{~K}$ or so. The 
calculation of the spot temperature will not therefore be

seriously affected by assuming the hemisphere visible at wave maximum to be spot-free.

The unreddened $\left(V-R_{J}\right)$ colour indices at wave maximum and wave minimum yield amplitudes of variation in the Johnson $\mathrm{V}$ and $\mathrm{R}$ bands

$$
\begin{aligned}
& \Delta M_{V}=0.297 \pm 0.010 \\
& \Delta M_{R}=0.238 \pm 0.010
\end{aligned}
$$

Substituting these values into equation (2.15) and solving iteratively, the difference between the colour indices of the photosphere and the spot is found to be

$$
D=(V-R)_{\text {star }}-(V-R)_{\text {spot }}=-0.48 \pm 0.05
$$

Using the $(\mathrm{V}-\mathrm{R})$ o colour index of the at wave maximum and the relations of Johnson (1966), this yields a spot temperature of $3750 \pm 100 \mathrm{~K}$, and an effective spot area $\alpha=0.32 \pm-.02$, from Eq. 2.15 .

These results are very similar to those obtained by Vogt (1981) for the single-lined RS CVn binary II Peg, whose 1977 lightcurve yielded $\mathrm{T}_{\text {photosphere }}=4600 \mathrm{~K}$, $\mathrm{T}_{\text {spot }}=3400 \pm 100 \mathrm{~K}$, and $\alpha=0.37 \pm 0.03$. It thu's seems from these observations that the temperature and extent of the dark region giving rise to the photometric wave in HD 32918 are similar to those observed in the RS CVn binaries. The high equatorial rotation rate observed in this star and the RS CVn-like nature of its spectrum also suggest that its behaviour is very similar to that seen in the RS CVn binaries. However, the lack of any observable radial velocity variation provides strong evidence that HD 32918 is not a binary, but a probable new member of the FK Comae class of single, rapidly rotating late type giants with RS CVn-like characteristics. 
$4.5 \quad \mathrm{HD} 34802$

The presence of $\mathrm{Ca}$ II $\mathrm{H}$ and $\mathrm{K}$ emission in the spectrum of HD 34802 has been noted by Bidelman and MacConnell (1973) and Houk and Cowley (1975), who classify its spectrum as KI IIIP and its emission strength as moderate.

\section{5 .1 Radial Velocity Observations}

A single spectrum of the $\mathrm{Mg} I \mathrm{~b}$ line region in $\mathrm{HD}$ 34802 was obtained at HJD 2444539.15 , giving a heliocentric radial velocity of $-13.4 \pm 3.0 \mathrm{~km} \mathrm{~s}^{-1}$. The c.c.f. showed a single, narrow peak, indicating that rotational smearing of the spectrum amounted to no more than $v_{e} \sin i \simeq 20 \mathrm{~km} \mathrm{~s}^{-1}$.

\subsubsection{Photometric observations}

BVR $_{C} I_{C}$ photometry of HD 34802 was obtained at MJUO during 1979 October, and 1980 January and February. Variations in $V$ with a total amplitude of at least 0.06 magnitude were observed, using HD 34297 as the comparison star.

An attempt was made at estimating a rotation period for this star, by assuming the variation to be sinusoidal and constructing a periodogram using the method of Gieseking (1981). Analysis of the aliasing structure in the periodogram (Fig. 2.2 (b) using the guidelines described in section 2.4.4, suggested that the minimum labelled $P_{0}$ in Fig.2.2 corresponded to the most likely rotation period, $\mathrm{P}_{\mathrm{O}}=20.45 \pm 0.06$ days.

The $\mathrm{V}$ photometric observations, folded with the above rotation period, are plotted against rotational phase in Fig. 4.5 .1 .

The colour indices obtained for HD 34802 (see Table 2.3) are consistent with a ko/I III/IV spectral type. 


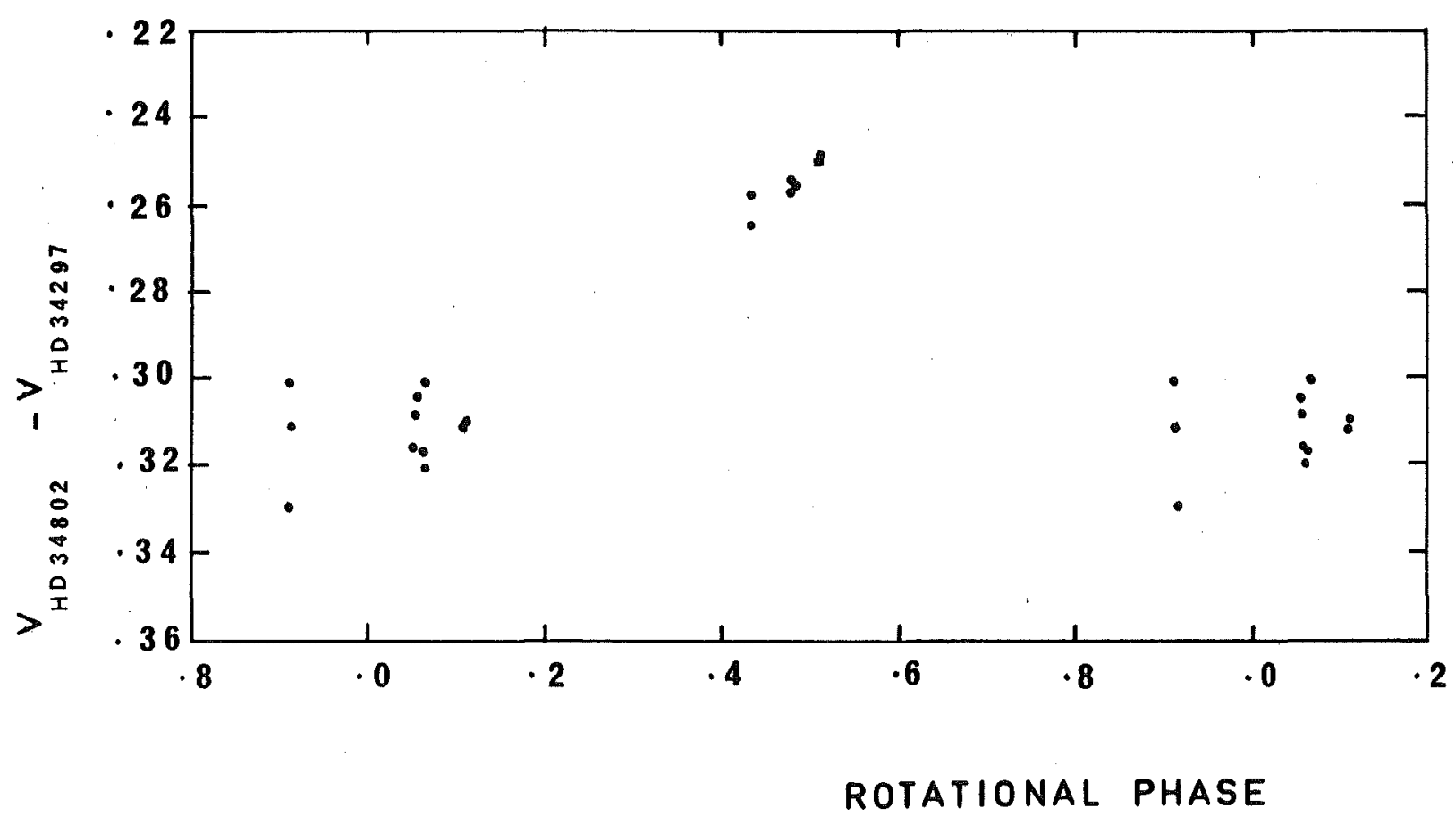

Fig. 4.5.1 V lightcurve of HD 34802, 1979 October to 1980 February. The phasing is arbitrary and the observations are folded with $P=20.45$ days. 
4.6 HD 36705

HD 36705 is noted as a Ca II $\mathrm{H}$ and $\mathrm{K}$ emitter by both Bidelman and MacConnell (1973) and Houk and Cowley (1975), who classify. its spectrum as KI IIIp. The results of high resolution spectroscopy of this star at the Mg I b lines and $\mathrm{H} \alpha$ are described in a separate paper by Collier (1982) . Pakull (1981) carried out an extensive photometric investigation of this object during 1980, following its identification as the most likely optical counterpart of a compact, flaring X-ray source detected by the EINSTEIN Observatory during a 1979 survey of the LMC. The star has been tentatively identified as a new member of the FK Comae class (Collier, 1982). A possible radio detection of HD 36705 was obtained with the $64 \mathrm{~m}$ radio telescope of the Australian National Radio Astronomy Observatory, Parkes, N.S.W., during $1981 \mathrm{July}$ (Collier, Haynes, Slee, Wright and Hillier, 1982). Photometric observations of HD 36705 obtained at MJUO during 1980 September, which have not been published, are presented here.

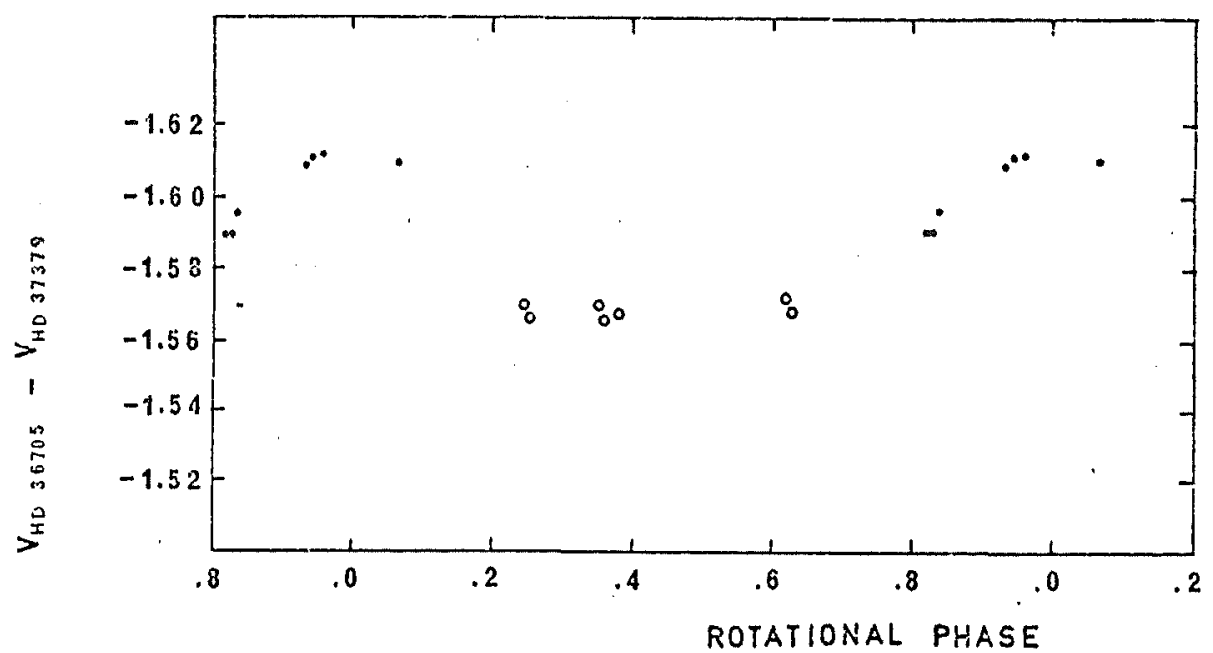

Fig. 4.6.1 V lightcurve of HD 36705, 1980 september. The ephemeris used was HJD $=2444296.575+0.51423 \mathrm{E}$. 
4.6.1 Photometric Observations

Photoelectric photometry of HD 36705 in the BVR $_{C}{ }^{I_{C}}$ system was obtained at MJUO on the nights of 1980 september 24 and 27.

The colour indices of HD 36705 and its comparison star, HD 34297 are given in Table 2.3. The colour indices of the program star are consistent with a G7/8 IV or KO $\mathrm{V}$ spectral type.

The observations show a variation with a total amplitude of $0.04 \mathrm{mag}$. in V. In Fig. 4.6.1 the V observations are plotted modulo phase according to the ephemaris of Pakull (1981), $\mathrm{HJD}=2444296.575+0.51423 \mathrm{E}$, measured from the epoch of spot minimum on 1980 February 27.

The variation is clearly modulated with the period determined by Pakull. The phases of wave maximum and minimum coincide with those obtained by Pakull during 1980 october. Pakull's earlier lightcurve is "flat-topped", with an amplitude of $0.12 \mathrm{mag}$. The later lightcurves show no evidence of a flat-topped maximum, and the amplitude of variation is considerably less. The data suggest that the initially compact spot-group observed by Pakull early in 1980 became more widely dispersed in longitude throughout the rest of that year, presumably due to the shearing effect of differential rotation in the star (Shore and Hall 1980). 
TABLE 4.7 .1

\begin{tabular}{|c|c|c|c|c|c|}
\hline $\begin{array}{l}\text { Date } \\
\text { U.T. }\end{array}$ & & $\begin{array}{l}\text { Exposure } \\
(\text { sec.) }\end{array}$ & $\begin{array}{c}\text { HJD } \\
2444000+\end{array}$ & $\begin{array}{l}\text { Heliocentric Rạilal } \\
\text { Mg I b }\left(\mathrm{km} \mathrm{s}^{-1}\right)\end{array}$ & $\begin{array}{l}\text { Velocity } \\
\qquad \mathrm{H} \alpha\end{array}$ \\
\hline 1980 & Oct. 26 & 550 & 589.042 & 22.8 & \\
\hline \multirow[t]{2}{*}{1980} & Nov .22 & 300 & 566.017 & 16.4 & \\
\hline & & $"$ & 566.051 & 14.8 & \\
\hline \multirow[t]{4}{*}{1980} & Nov. 23 & $"$ & 567.023 & 14.6 & \\
\hline & & $"$ & 567.061 & 14.2 & \\
\hline & & $"$ & 567.109 & 14.0 & \\
\hline & & $"$ & 567.163 & 17.5 & \\
\hline 1980 & Dec. 18 & 1090 & 593.16 & & 9.0 \\
\hline 1980 & Dec. 19 & 610 & 594.12 & & 6.2 \\
\hline 1980 & Dec. 20 & 430 & 595.04 & & 7.5 \\
\hline 1980 & Dec. 21 & 730 & 596.13 & & 2.6 \\
\hline
\end{tabular}

Table 4.7.1 Radial Velocities of HD 37434 from Mg I b and $\mathrm{H} \alpha$ spectra, MSO 1980.

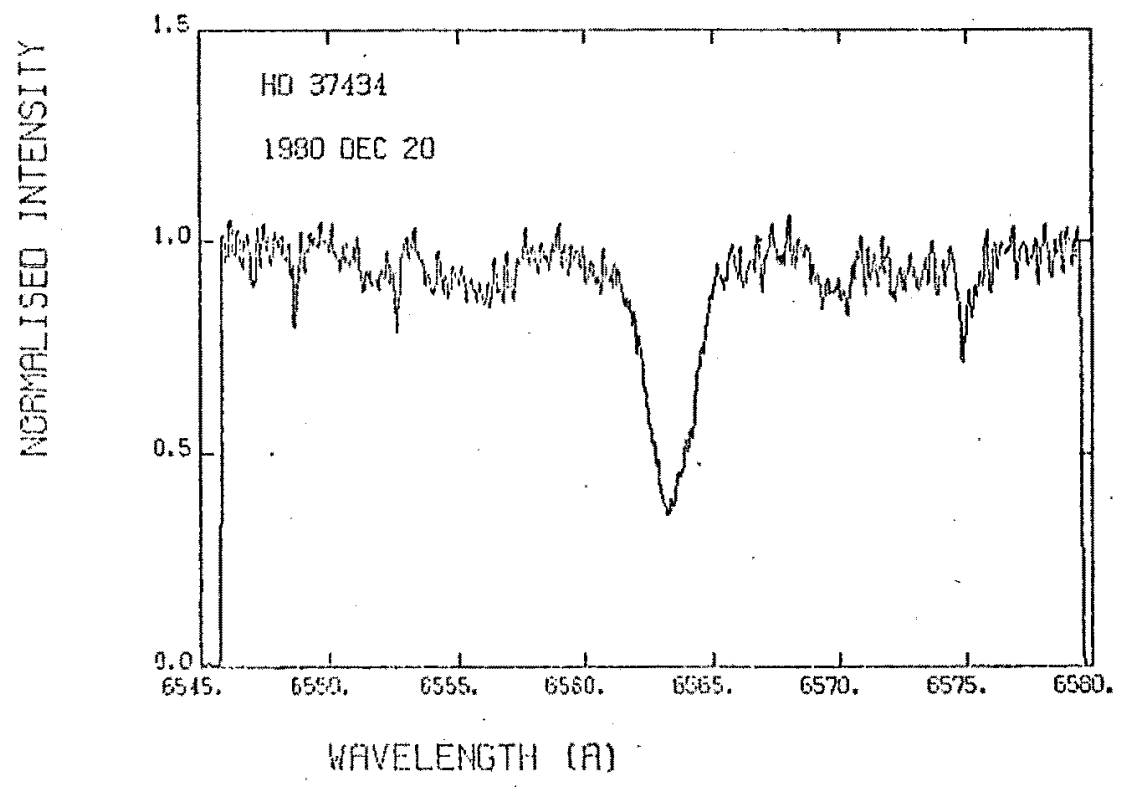

F1g. 4.7.1 Hol spectrum of HD 37434. 
$4.7 \quad$ HD $37434(=$ HR 1927)

HD 37434 appears on the list of southern $\mathrm{Ca}$ II $\mathrm{H}$ and $\mathrm{K}$ emitters by Bidelman and MacConnell (1973). "The spectral type is listed as KI III + F.

\section{7 .1 Radial Velocity Observations}

Seven spectra of the Mg I b line region in HD 37434 were obtained at MSO during 1980 October and November. Cross-correlation of the spectra with the template spectrum of HR 141 produced a single peak, with moderately high $\left(\sim 40 \mathrm{~km} \mathrm{~s}^{-1}\right)$ rotational broadening. The radial velocities derived from the c.c.f. shifts are listed in Table 4.7.1.

\section{$4.7 .2 \quad \mathrm{H} \alpha$ Spectra}

Four high-resolution spectra of the $H \alpha$ region in HD 37434 were obtained during 1980 December. H $\alpha$ itself appeared as an apparently normal absorption feature, somewhat broadened by rotation (Fig. 4.7.1). The smearing of the metallic line spectrum corresponded to $v_{e} \sin i \geqslant 35 \mathrm{~km} \mathrm{~s}^{-1}$, in agreement with the result obtained from the Mg I b data. The radial velocities of the metallic line spectrum are listed in Table 4.7.1, and confirm that HD 37434 is a spectroscopic binary. Insufficient data were available for any period determination to be made for this system. 
HD 37847 appears in the list by Bidelman and MacConnell (1973) of southern hemisphere Ca II H and $\mathrm{K}$ emitters. F. Fekel has observed the $\mathrm{Ca}$ II $\mathrm{H}$ and $\mathrm{K}$ line region in $\mathrm{HD} 37847$ (B.Bopp, private communication, 1981), and has found the star to be a double-lined spectroscopic binary.

\subsubsection{H H spectra}

Three $\mathrm{H}_{\alpha}$ spectra were obtained of HD 37847 during 1980 December. The spectra (Fig. 4.8.1) show Ha as a shallow absorption feature, with a weak emission feature above continuum level displaced some $100 \mathrm{~km} \mathrm{~s}^{-1}$ to blueward of the line centre. The metallic lines near $\mathrm{H} \alpha$ are rotationally smeared, with $v_{e} \sin i=12 \pm 3 \mathrm{~km} \mathrm{~s}^{-1}$. No evidence of a secondary spectrum was seen; the spectrum observed at $H \alpha$ is presumably that of the cooler, chromospherically active component. The presence of the blue-shifted $\mathrm{H} \alpha$ emission feature suggests a high level of surface activity, with some net mass outflow.

The radial velocities of the metallic line spectrum are listed in Table 4.8.1.

TABLE 4.8 .1

$\begin{array}{lccc}\text { Date } & \text { Exposure } & \text { HJD } & \text { Heliocentric Radial Velocity } \\ \text { U.T. } & \text { (sec.) } & 2444000+ & \left(\mathrm{km} \mathrm{s}^{-1}\right)\end{array}$

1980 Dec.19 1090

594.22

27.2

1980 Dec. $20 \quad 1630$

595.05

19.0

1980 Dec.21 2950

596.15

10.2

Table 4.8.1 Radial Velocities of HD 37847 from Ha spectra, MSO, 1980 . 


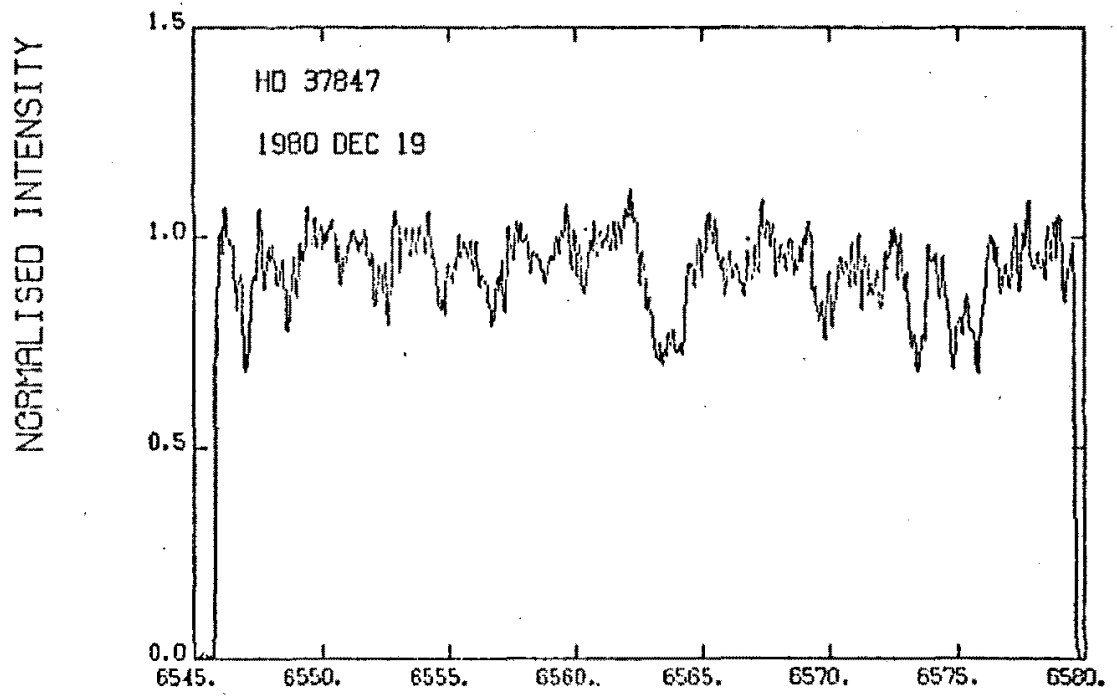

WRVELENGTH (A)

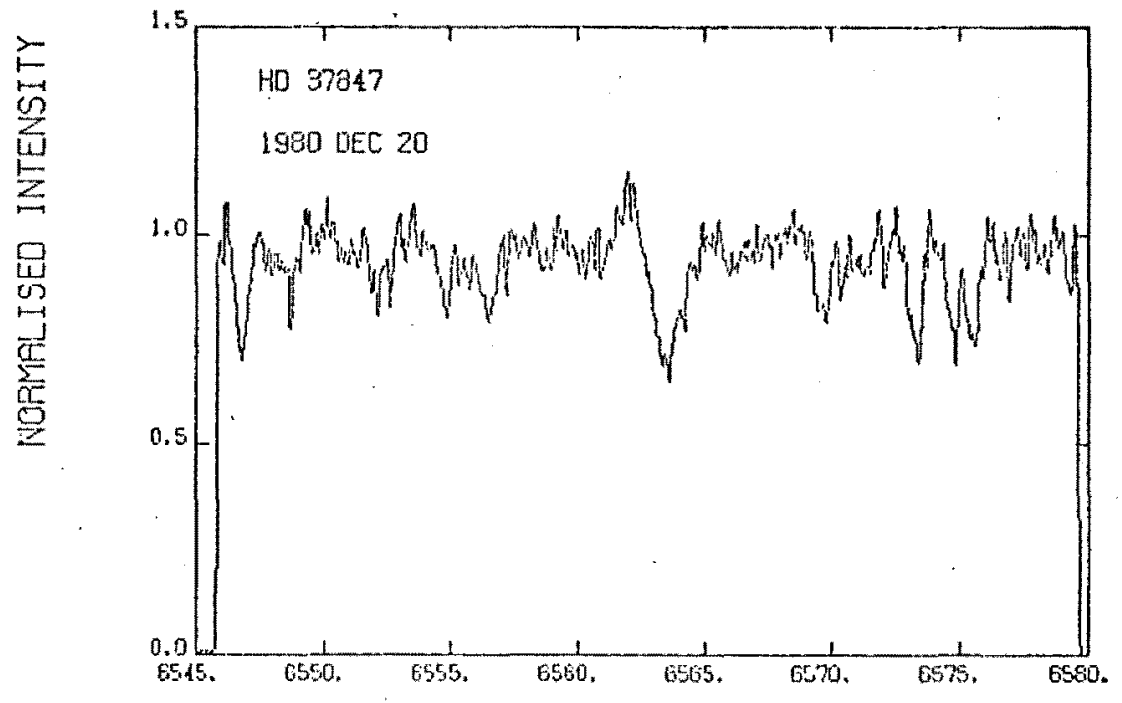

WAVELENGTH (A)

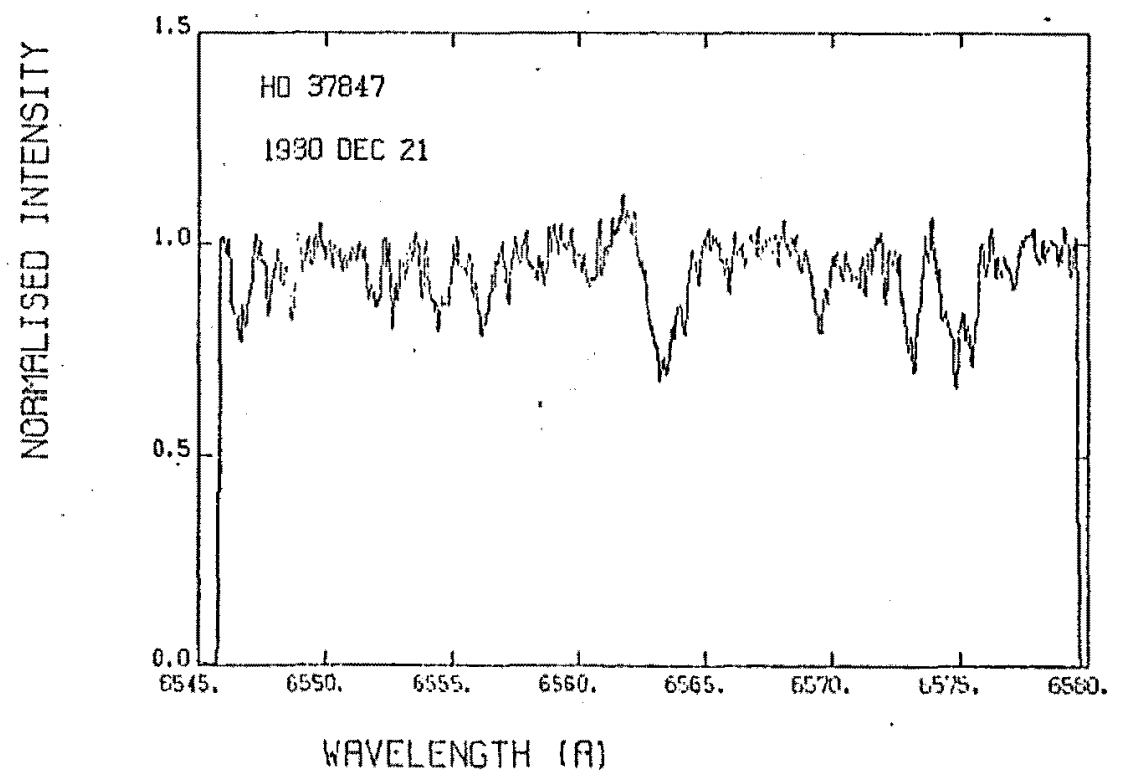


Houk and Cowley (1975) assign a spectral type of F7 IV to this star. No evidence of Ca II emission has been observed in its spectrum; however, Weiler and Stencel (1979) classify HD 39937 as a possible RS CVn candidate on the strength of its known variability.

\subsubsection{Radial Velocity Observations}

Seven spectra of the Mg I b line region in HD 39937 obtained at MSO during 1980 October and November showed HD 39937 to be a single-lined spectroscopic binary. The radial velocities are listed in Table 4.9 .1 .

\section{$\underline{4.9 .2 \mathrm{H} \alpha \text { Spectra }}$}

Four $\mathrm{H} \alpha$ spectra of HD 39937 were obtained at MSO during 1980 December. The spectra all show an apparently normal, single late $F$ spectrum (Fig. 4.9.1), with no: evidence for rotational smearing in excess of $v_{e} \sin i \simeq$ $8 \mathrm{~km} \mathrm{~s}^{-1}$.

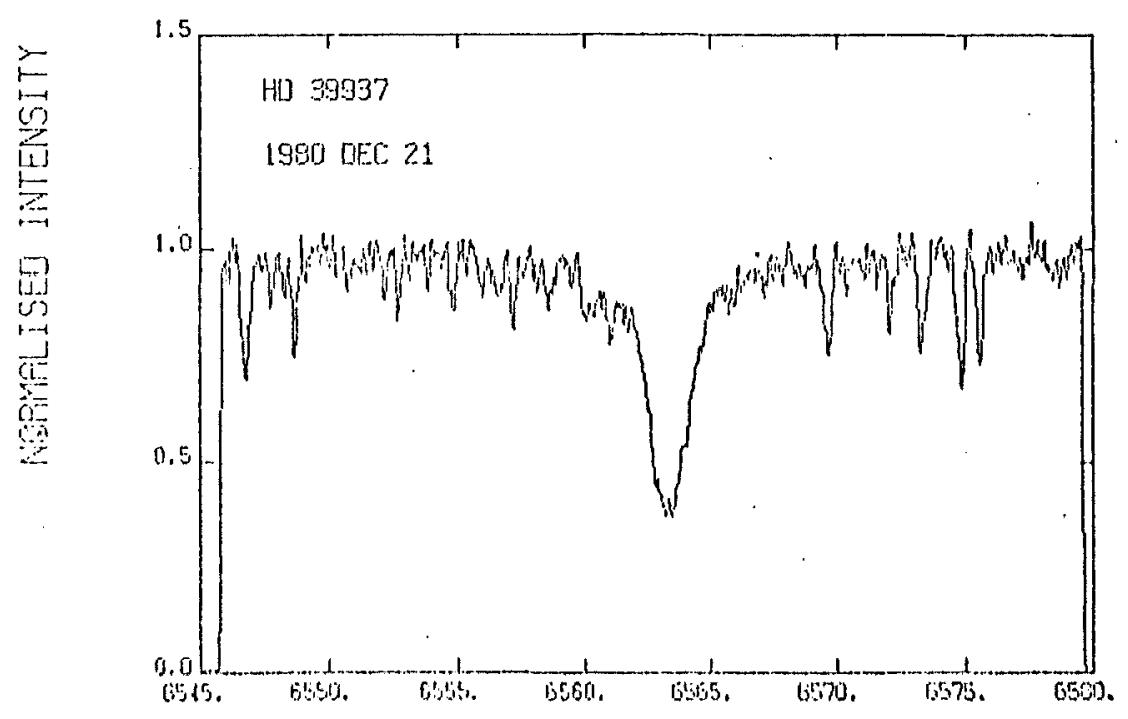

WEVELENTH (B)

Fig. 4.9.1 H $\mathrm{H} \alpha$ spectrum of HD 39937. 
TABLE 4.9 .1

\begin{tabular}{|c|c|c|c|c|}
\hline \multicolumn{2}{|c|}{$\begin{array}{l}\text { Date } \\
\text { U.T. }\end{array}$} & $\begin{array}{c}\text { Exposure } \\
\text { (sec.) }\end{array}$ & $\begin{array}{c}\text { HJD } \\
2444000+\end{array}$ & $\begin{array}{l}\text { Heliocer } \\
\quad \text { Mg I b }\end{array}$ \\
\hline 1980 & Oct. 26 & 300 & 539.217 & 59.8 \\
\hline 980 & Nov. 22 & $"$ & 566.002 & -11.9 \\
\hline & $"$ & $"$ & 566.044 & -13.2 \\
\hline 80 & Nov. 23 & $"$ & 567.008 & -11.5 \\
\hline & $"$ & $"$ & 567.048 & -10.1 \\
\hline & $n$ & $"$ & 567.094 & -8.5 \\
\hline & $"$ & $"$ & 567.149 & -9.7 \\
\hline
\end{tabular}

1980 Dec. $18 \quad 670$

593.15

38.2

1980 Dec. 19550

594.13

31.6

1980 Dec. $20 \quad 550$

595.07

26.3

1980 Dec. $21 \quad 850$

596.18

21.8

Table 4.9.1 Radial Velocities of HD 39937 from Mg I b and $\mathrm{H} \alpha$ spectra, MSO 1980. 
The presence of $\mathrm{Ca}$ II $\mathrm{H}$ and $\mathrm{K}$ emission in the spectrum of HD 42504 has been noted by Bidelman and MacConnell (1973). The spectral type is listed as G8 III + F.

\section{$\underline{4.10 .1 \quad \mathrm{H} \alpha \text { Spectra }}$}

Three spectra of the Ha region in HD 42504 were obtained at MSO during 1980 December. The smearing of the metallic line spectrum corresponds to an equatorial rotation speed $v_{e} \sin i \simeq 15 \mathrm{~km} \mathrm{~s}^{-1}$, and $\mathrm{H} \alpha$ appears as an absorption feature (Fig. 4.10.1). The radial velocities of the metallic line spectrum are Iisted in Table 4.10.1; although the observed variation is small, the high equatorial rotation indicates that HD 42504 is likely to be a single-lined spectroscopic binary.

TABLE $\quad 4.10 .1$

$\begin{array}{lccc}\begin{array}{l}\text { Date } \\ \text { U.T. }\end{array} & \begin{array}{c}\text { Exposure } \\ \text { (sec.) }\end{array} & \begin{array}{c}\text { HJD } \\ 2444000+\end{array} & \begin{array}{c}\text { Heliocentric Radial Velocity }\left(\mathrm{km} \mathrm{s}^{-1} \text { ) }\right. \\ \text { H }\end{array} \\ 1980 \text { Dec.19 } & 2000 & 594.15 & 74.7 \\ 1980 \text { Dec.20 } & 2110 & 595.20 & 71.4 \\ 1980 \text { Dec.21 } & 1810 & 596.20 & 67.3\end{array}$

Table 4.10.J. Radial velocities of HD 42504 from $H \alpha$ spectra, MSO, 1980. 


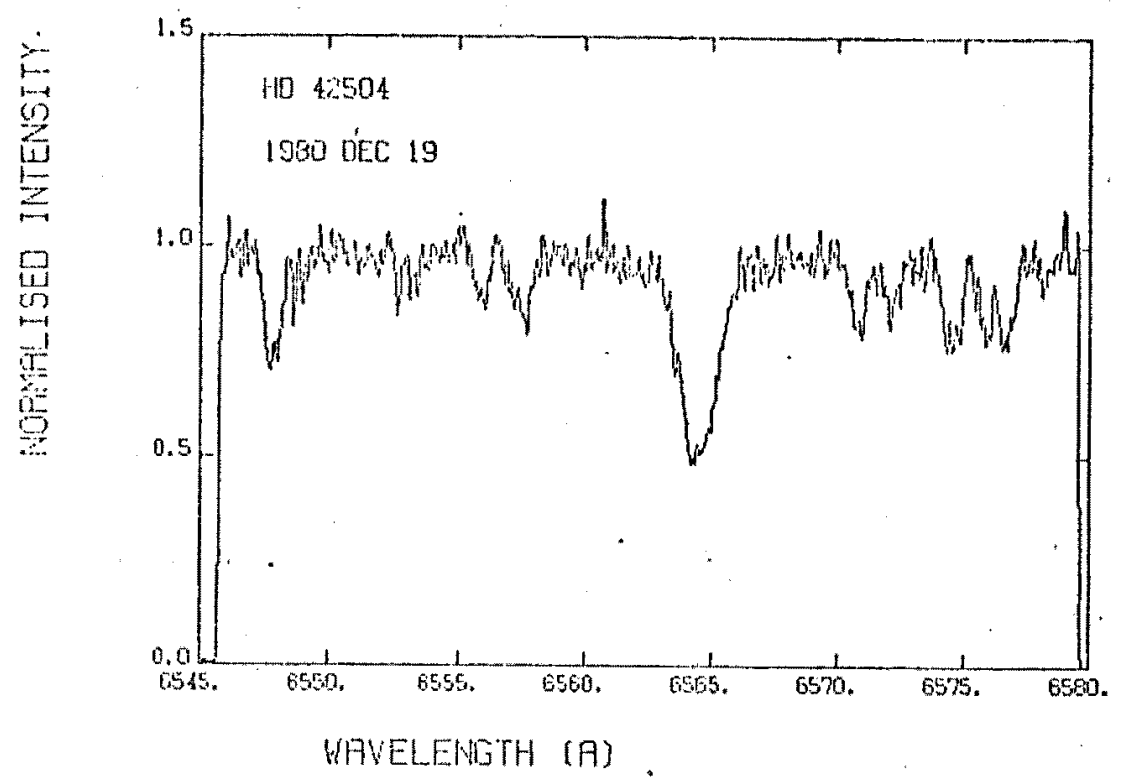

Fig. 4.10.1 H $\alpha$ spectrum of HD 42504 .

\section{$4.11 \quad H D \quad 46697$}

Bidelman and MacConnell (1973) and Houk and Cowley (1975) have noted the presence of moderately strong Ca II $\mathrm{H}$ and $\mathrm{K}$ emission in the spectrum of HD 46697. Houk and Cowley classify its spectrum as $\mathrm{KI}$ III/IVp.

\subsubsection{Radial Velocity Observations}

A single spectrum of the Mg I b line region in HD 46697, obtained at MSO on 1980 October 26, shows a single set of absorption lines. The radial velocity is included in Table 4.11.1.

\section{$4.11 .2 \quad \mathrm{H} \alpha$ Spectra}

Four $H_{\alpha}$ spectra of HD 46697 were obtained at MSO during 1980 December and 1981 February. H $\alpha$ appears as an absorption feature; the nearby metallic line spectrum shows 

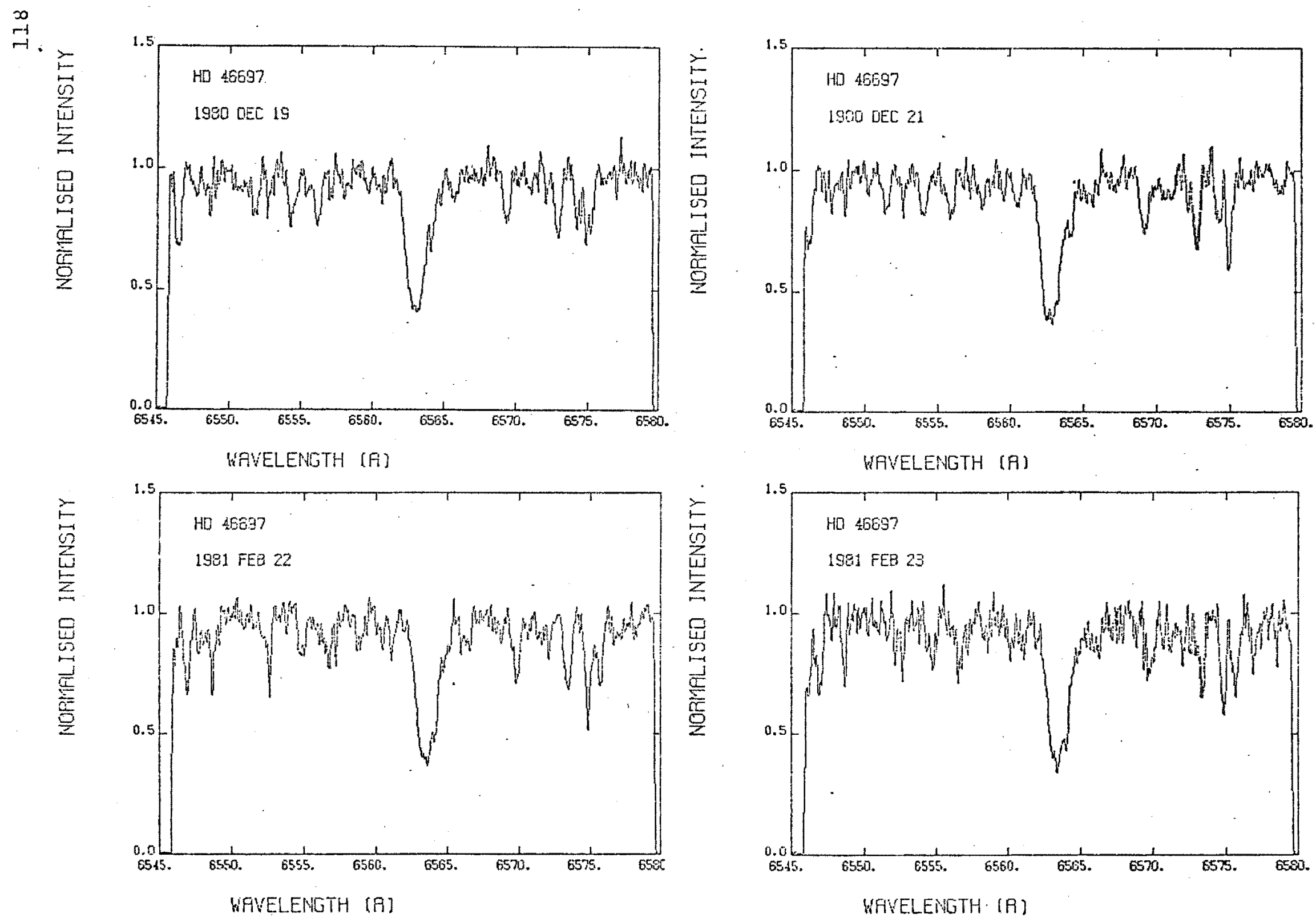
rotational smearing corresponding to $v_{e}$ sin $i=10 \pm 3 \mathrm{~km} \mathrm{~s}^{-1}$ and radial velocity variations with a total amplitude of at least $30 \mathrm{~km} \mathrm{~s}^{-1}$. The spectra are presented in Fig. 4.11.1, and the radial velocities are listed in Table 4.11.1.

\subsubsection{Ca II $\mathrm{H}$ and $\mathrm{K}$ Spectra}

Eight spectra of the Ca II K line region in HD 46697 were obtained at sso during 1981 March and April, and three spectra of the $\mathrm{H}$ and $\mathrm{K}$ Iine region were obtained at MSO during 1981 March.

Cross-correlation of the absorption spectrum with a template as described in section 3.5 .3 revealed no sign of a secondary absorption spectrum at this wavelength. The radial velocities derived from the c.c.f. lags, as well as the directly-measured velocities of the $H$ and $K$ emission peaks (see Section 3.5.3) are listed in Table 4.11.1.

The radial velocity variations are shared by both the absorption and emission spectra, indicating that the chromospheric activity is associated with the visible component.

The orbital period (13. $\overline{6}$ days) was determined using Gieseking's (1981) method (Section 3.7). The preliminary radial velocity solution, assuming a circular orbit, is presented in Table 4.11.2. Figs. 4.11.2 and 4.11.3 depict the periodogram and radial velocity curve for HD 46697.

The half-width of the Ca II K emission core is $W_{0}=0.90 \pm 0.05 \AA$. By assuming an effective temperature appropriate to the KI III/IV spectral type given by Houk and Cowley (1975), a surface gravity $\log g=2.6 \pm 0.5$ was calculated, as described in section 3.5.4.

The absolute $\mathrm{Ca}$ II $\mathrm{H}$ and $\mathrm{K}$ surface emission flux (Section 3.5.5) was calculated to be $F\left(K_{1}\right)+F\left(H_{1}\right)=2.0 \times 10^{6}$ erg $\mathrm{cm}^{-2} \mathrm{~s}^{-1}$. This value is uncertain by about $50 \%$, owing 
TABLE 4.11 .1

$\begin{array}{lccccccc}\text { Date } & \text { Exposure } & \text { HJD } & \text { Heliocentric Radial velocity } & \left(\mathrm{km} \mathrm{s}^{-1} \text { ) }\right. \\ \text { U.T. } & \text { (sec.) } & 2444000+ & \text { Mg I b } & \text { Ho } & 3920 \AA & \mathrm{K} & \mathrm{H} \\ & & & & \text { Abs } & \mathrm{Em} & \mathrm{Em}\end{array}$

\begin{tabular}{|c|c|c|c|c|c|c|c|c|c|}
\hline 1980 & Oct. & 26 & 300 & 539.159 & 5.5 & & & & \\
\hline 1980 & Dec. & 19 & 1510 & 594.17 & & 6.1 & & & \\
\hline 1980 & Dec. & 21 & 1800 & 596.22 & & -3.8 & & & \\
\hline 1981 & Feb. & 22 & 2770 & 658.05 & & 25.4 & & & \\
\hline 1981 & Feb. & 23 & 970 & 659.03 & & 22.6 & & & \\
\hline 1981 & Max. & 20 & 3600 & 683.92 & & & 23.0 & 28.7 & \\
\hline 1981 & Mar. & 21 & 3600 & 684.90 & & & 27.1 & 20.2 & \\
\hline 1981 & Mar. & 24 & 1510 & 687.91 & & & 10.8 & 5.8 & 11.7 \\
\hline 1981 & Mar. & 25 & 3600 & 688.92 & & & 0.3 & 1.8 & 7.2 \\
\hline 1981 & Mar. & 26 & 2110 & 689.90 & & & -5.7 & -4.5 & -3.1 \\
\hline 1981 & Apr. & 15 & 3600 & 709.90 & & & 35.6 & 32.5 & \\
\hline 1981 & Apr. & 16 & $"$ & 710.89 & & & 25.5 & 26.1 & \\
\hline 1981 & Apr. & 17 & $"$ & 711.89 & & & 27.8 & 27.5 & \\
\hline 1981 & Apr. & 18 & $"$ & 712.89 & & & 28.3 & 29.7 & \\
\hline 1981 & Apr & 19 & $"$ & 713.88 & & & 24.2 & 23.2 & \\
\hline 1981 & Apr. & 20 & $"$ & 714.88 & & & 18.0 & 20.4 & \\
\hline
\end{tabular}

Table 4.11.1 Radial Velocities of HD 46697 from Mg I b, $\mathrm{H} \alpha$ and Ca II spectra, MSO and SSO 1980/81. 
곡

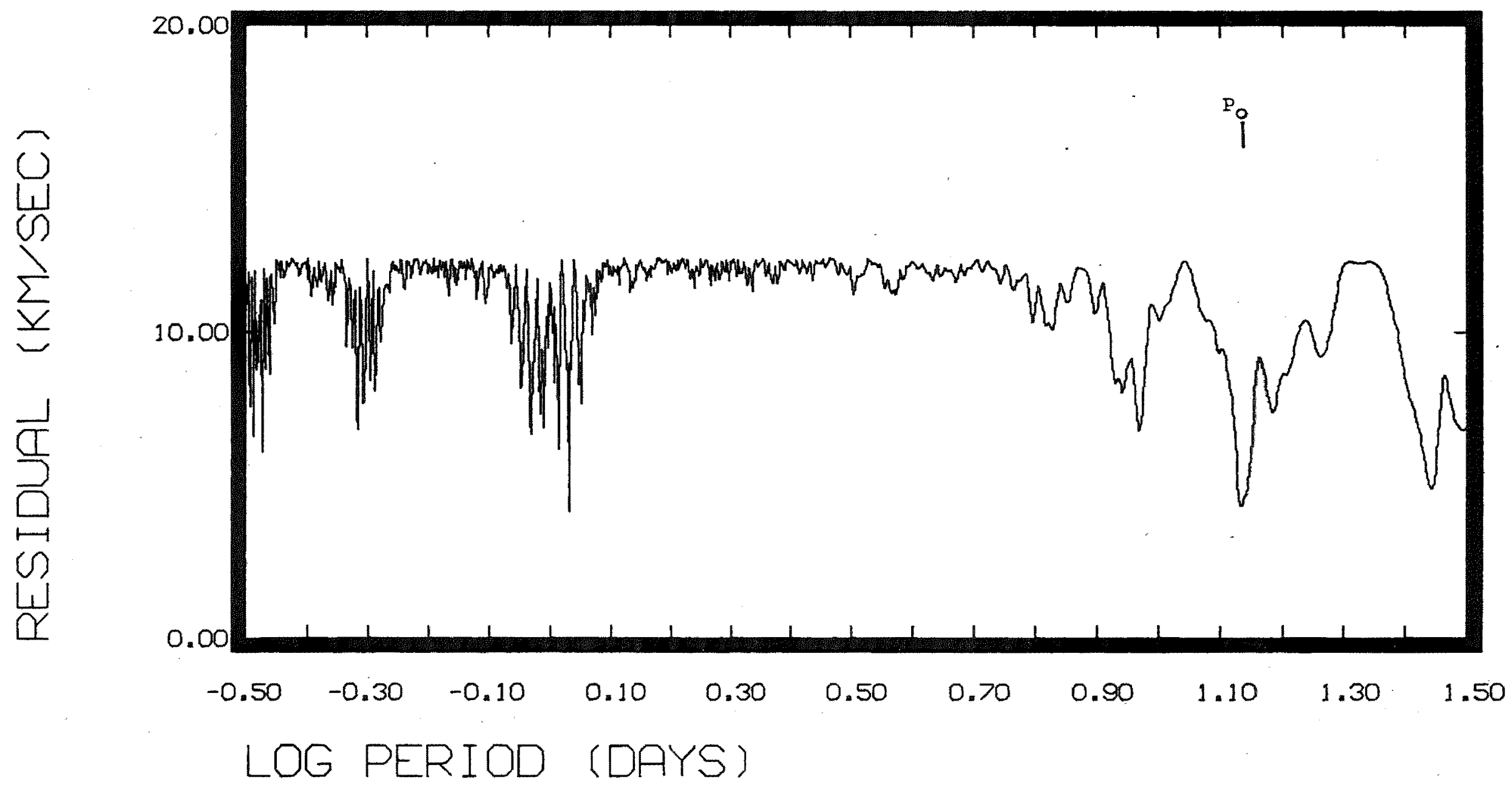

Fig. 4.11.2 Periodogram for HD 46697 radial velocities. 
mainly to the fact that the $\left(V-R_{J}\right)$ colour index was not measured directly, but estimated from Houk and Cowley's spectral type. The $\mathrm{K}$ emission fluxes measured at sso during 1981 April are plotted in Fig. 3.2, and show a net increase of about $15 \%$ during the observing run. Unfortunately, the lack of photometry of this system does not allow determination of the period of any photometric variation which may be present, so the rotational dependence of the $\mathrm{Ca}$ II $\mathrm{K}$ flux cannot be ascertained.

\subsection{1 .4 Discussion}

Comparison of the surface gravity and the spectral type with Iben's evolutionary tracks in the $\left(\log g, \log \mathrm{T}_{e}\right)$ plane indicates that the visible component is probably an object with $M_{1}=3.0 \pm 0.8 \mathrm{M}_{\odot}$ which has evolved to the base of the giant branch. The radius would lie in the range $R_{1}=13+3 R_{\odot}$ for such a star, depending on the mass. This has important implications as far as the mass ratio $q=M_{2} / M_{1}$ of the binary is concerned. Iben's evolutionary tracks in the $\left(\log \frac{L}{L_{0}}, \log T_{e}\right)$ plane show that an object in this mass range undergoes only a small variation in bolometric luminosity during its evolution from the main sequence to the base of the giant branch. Indeed, if HD 46697 has a mass ratio close to unity, the less evolved component should be a hotter star with a bolometric luminosity comparable to that of its cooler companion. The spectrum of the secondary should thus be detectable in the blue part of the spectrum.

The absence of any detectable secondary absorption in the Ca II spectra thus suggests a mass ratio $q \leqslant 0.75$. The Roche lobe radius of the primary does not vary greatly with $q$, and for $0.2 \leqslant q \leqslant 0.7, M_{1}=3.0 \pm 0.8 M_{\odot}$, we find that 
$R_{L}=17 \pm 3 R_{\odot}$, depending mainly on the primary mass.

The ratio $R_{1} / R_{L}$ is probably greater than 0.5 , indicating that the primary is highly likely to be co-rotating with its companion. A photometric study of this system should be made in order to determine the colour indices and test whether the primary is in synchronous rotation. Refined measures of the surface gravity, the effective temperature and the rotational smearing profile may then be used to obtain a reasonably precise value for the axial (and hence presumably the orbital) inclination of the system. This in turn should yield a more useful set of constraints on the mass of the secondary component.

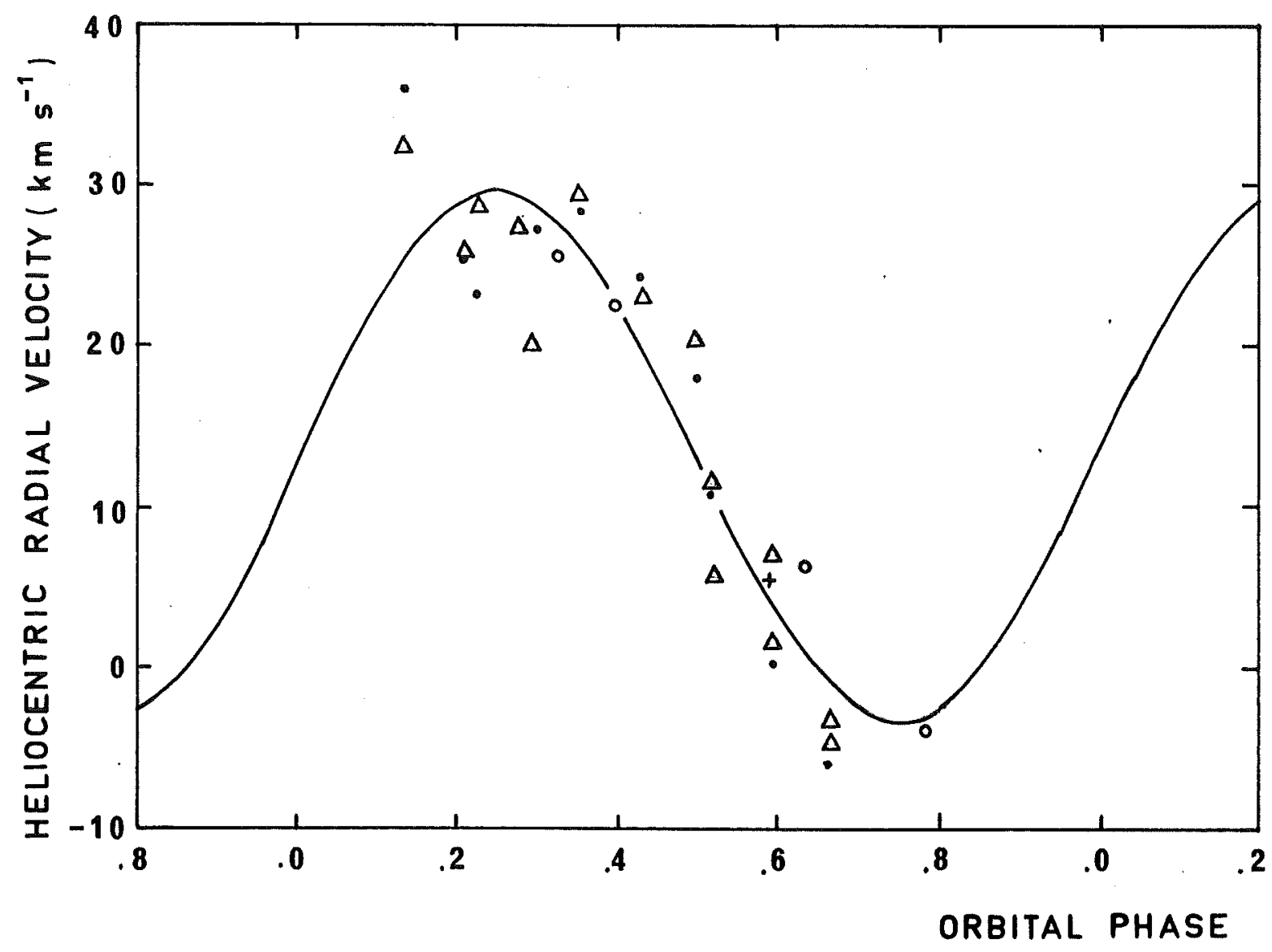

Fig. 4.11.3 Radial velocities of HD 46697 from Mg I b spectra (crosses), H $\alpha$ spectra (open circles), absorption spectrum near $3920 \AA$ (dots), $\mathrm{H}$ and $\mathrm{K}$ emission (triangles). The solid curve is a least-squares fit to the data, assuming a circular orbit. 

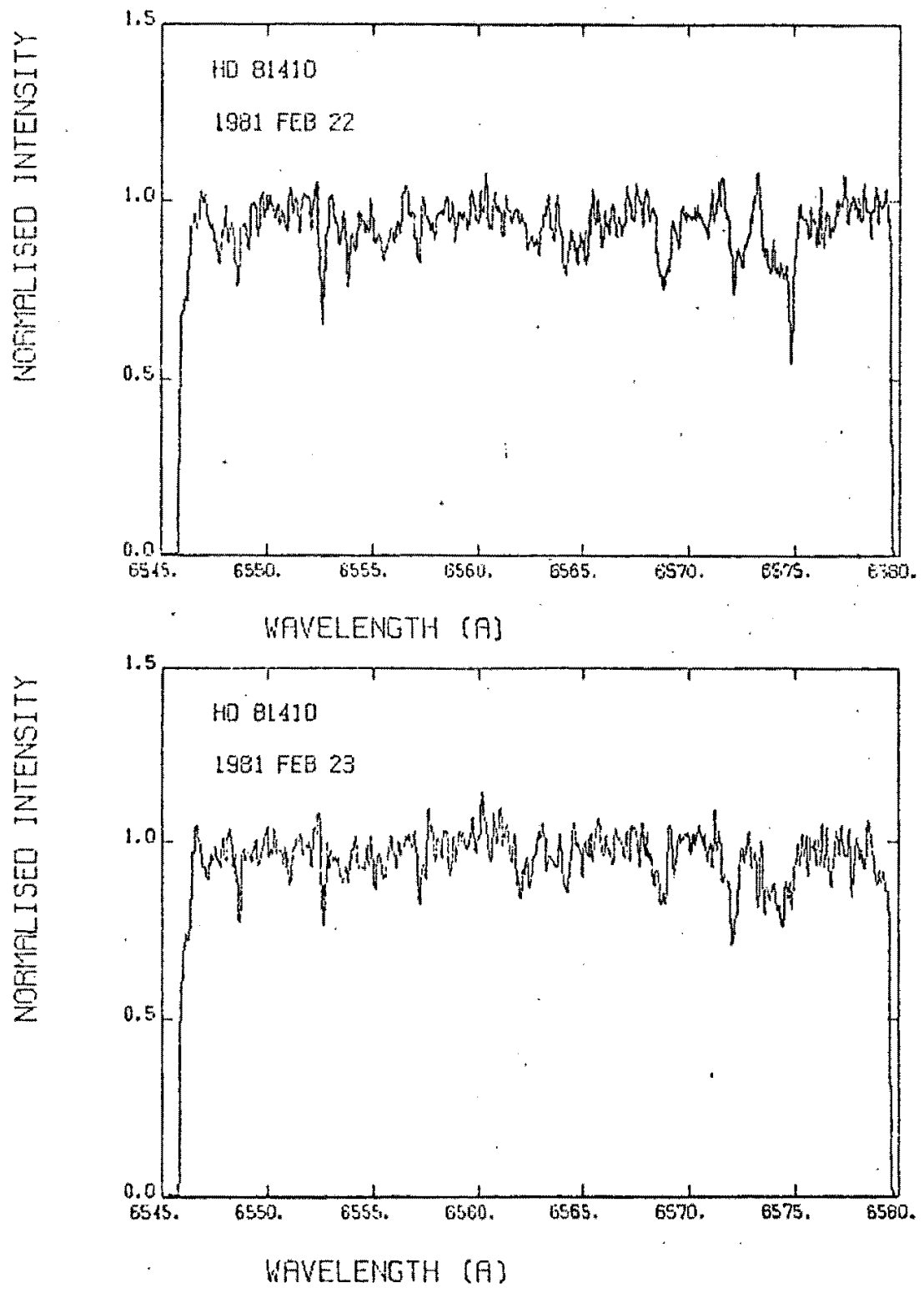

Fig. 4.12.1 Ho spectra of HD 81410. 
The presence of $\mathrm{Ca}$ II $\mathrm{H}$ and $\mathrm{K}$ emission in the spectrum of HD 81410 is noted by Bidelman and MacConnell (1973), who also state that the Balmer lines appear to be filled in. Eggen (1973) derives a period of 25.4 days for the photometric variability, based upon observations made during 1971 and 1972. In the same paper, Eggen lists several radial velocities obtained by P. Wayman and D.H.P. Jones. Wayman, in the same communication to 0.J. Eggen, gives the spectral type of $\mathrm{HD} 81410$ as $\mathrm{KI} \mathrm{IV}$.

\section{$4.12 .1 \quad \mathrm{H} \alpha$ Spectra}

Two Ha spectra of HD 81410 were obtained at MSO on the nights of 1981 February 22 and 23. Both show a single metallic line spectrum, with variable radial velocity and rotational smearing corresponding to $v_{e} \sin i=15 \pm 5 \mathrm{~km} \mathrm{~s}^{-1}$. $\mathrm{H} \alpha$ is filled to continuum level with emission in both spectra (Fig. 4.12.1). The radial velocities of the metallic line spectrum are listed in Table 4.12.1.

\section{$4.12 .2 \quad \mathrm{Ca}$ II $\mathrm{H}$ and $\mathrm{K}$ Spectra}

Three coudé spectra of the $\mathrm{Ca}$ II $\mathrm{H}$ and $\mathrm{K}$ line region in HD 81410 were obtained at MSO during 1981 March, and ten échelle spectra of the $\mathrm{K}$ line region were obtained at sso during 1981 'March and April.

The cross-correlations of the absorption spectrum near $3920 \AA$ with the template spectrum (see Section 3.5.3) revealed no secondary peaks, indicating that the binary is singlelined at this wavelength as well as in the red part of the spectrum. The $\mathrm{H}$ and $\mathrm{K}$ emission cores were found to share the radial velocity variations of the absorption spectrum. 
TABLE 4.12. 1

\begin{tabular}{|c|c|c|c|c|c|}
\hline Date & Exposure & HJD & Heliocentric & radial & velocity $\left(\mathrm{km} \mathrm{s}^{-1}\right)$ \\
\hline U.T. & (sec.) & $2444000+$ & $\begin{array}{l}3920 \AA \\
\text { Abs. }\end{array}$ & $\begin{array}{c}\mathrm{K} \\
\mathrm{Em}\end{array}$ & H. \\
\hline
\end{tabular}

\begin{tabular}{|c|c|c|c|c|c|c|c|}
\hline 1981 & Feb. 22 & 2950 & 658.09 & -17.7 & & & \\
\hline 1981 & Feb. 23 & 3130 & 659.18 & -32.0 & & & \\
\hline 1981 & Mar. 20 & 1573 & 684.04 & & -20.0 & -21.2 & \\
\hline 1981 & Mar. 21 & 3600 & 684.96 & & -36 & -34.7 & \\
\hline 1981 & Mar. 22 & 3600 & 685.98 & & -47.9 & -47.5 & \\
\hline 1981 & Mar. 24 & 2000 & 687.96 & & -42.1 & -46.8 & -49.8 \\
\hline 1981 & Mar. 25 & 3600 & 688.97 & & -37.1 & -42.1 & -33.1 \\
\hline 1981 & Max. 26 & 2110 & 689.93 & & -10.6 & -9.6 & -10.6 \\
\hline 1981 & Apr. 14 & 3600 & 708.96 & & -7.6 & -3.3 & \\
\hline 1981 & Apr. 15 & " & 709.96 & & -22.0 & -21.0 & \\
\hline 1981 & Apr. 16 & $"$ & 710.94 & & -40.8 & -39.6 & \\
\hline 1981 & Apr. 17 & $"$ & 711.94 & & -44.1 & -51.2 & \\
\hline 1981 & Apr. 18 & $"$ & 712.94 & & -43.9 & -48.0 & \\
\hline 1981 & Apr. 19 & $"$ & 713.93 & & -38.3 & -40.1 & \\
\hline 1981 & Apr. 20 & $"$ & 714.93 & & -32.4 & -26.0 & \\
\hline
\end{tabular}

Table 4.12.1 Radial velocities of HD 81410 from $\mathrm{H} \alpha$ and $\mathrm{Ca}$ II spectra, MSO and SSO, 1981. 


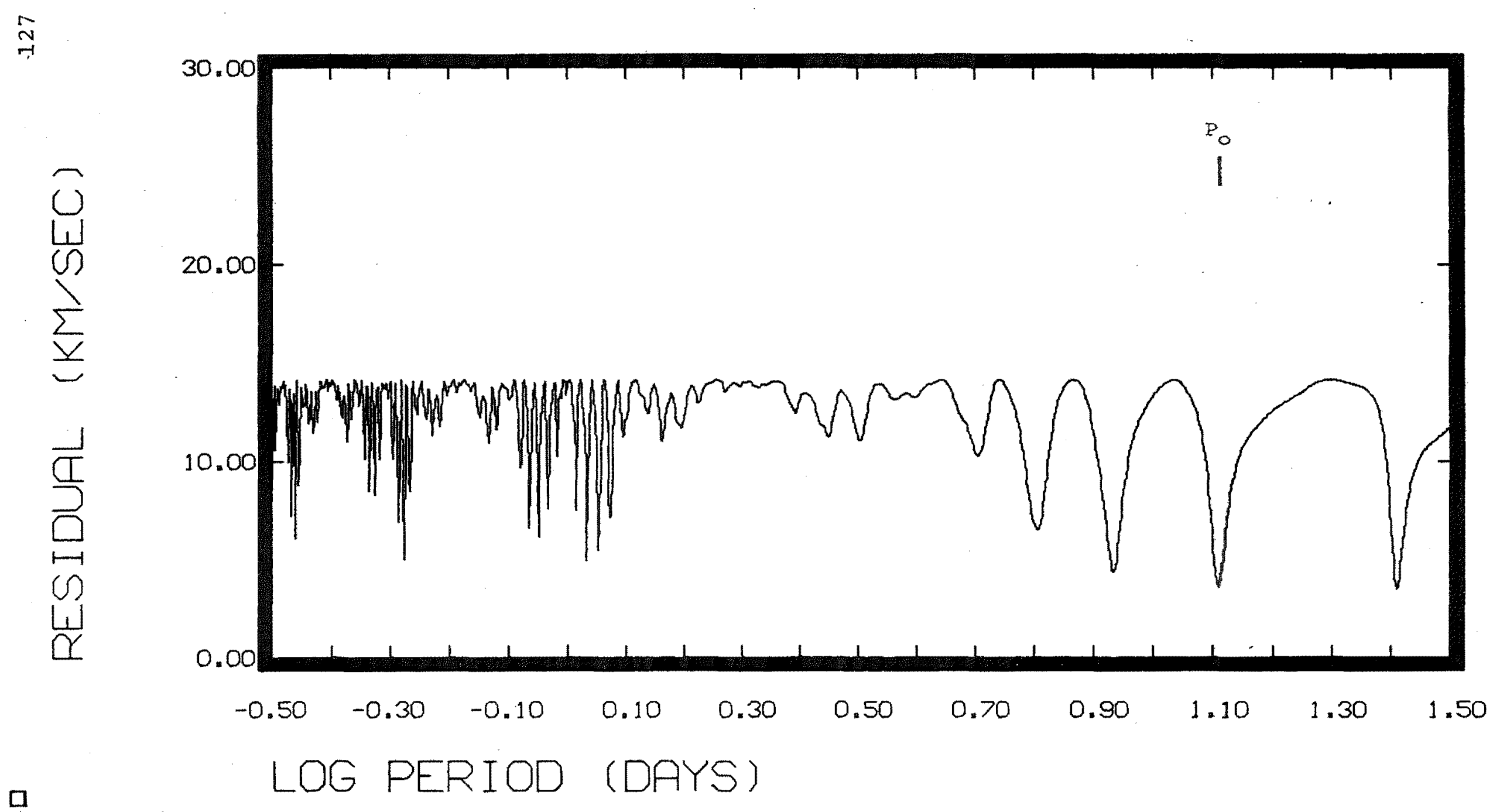

Fig. 4.12.2 Periodogram for $\mathrm{HD} 81410$ radial velocities. 

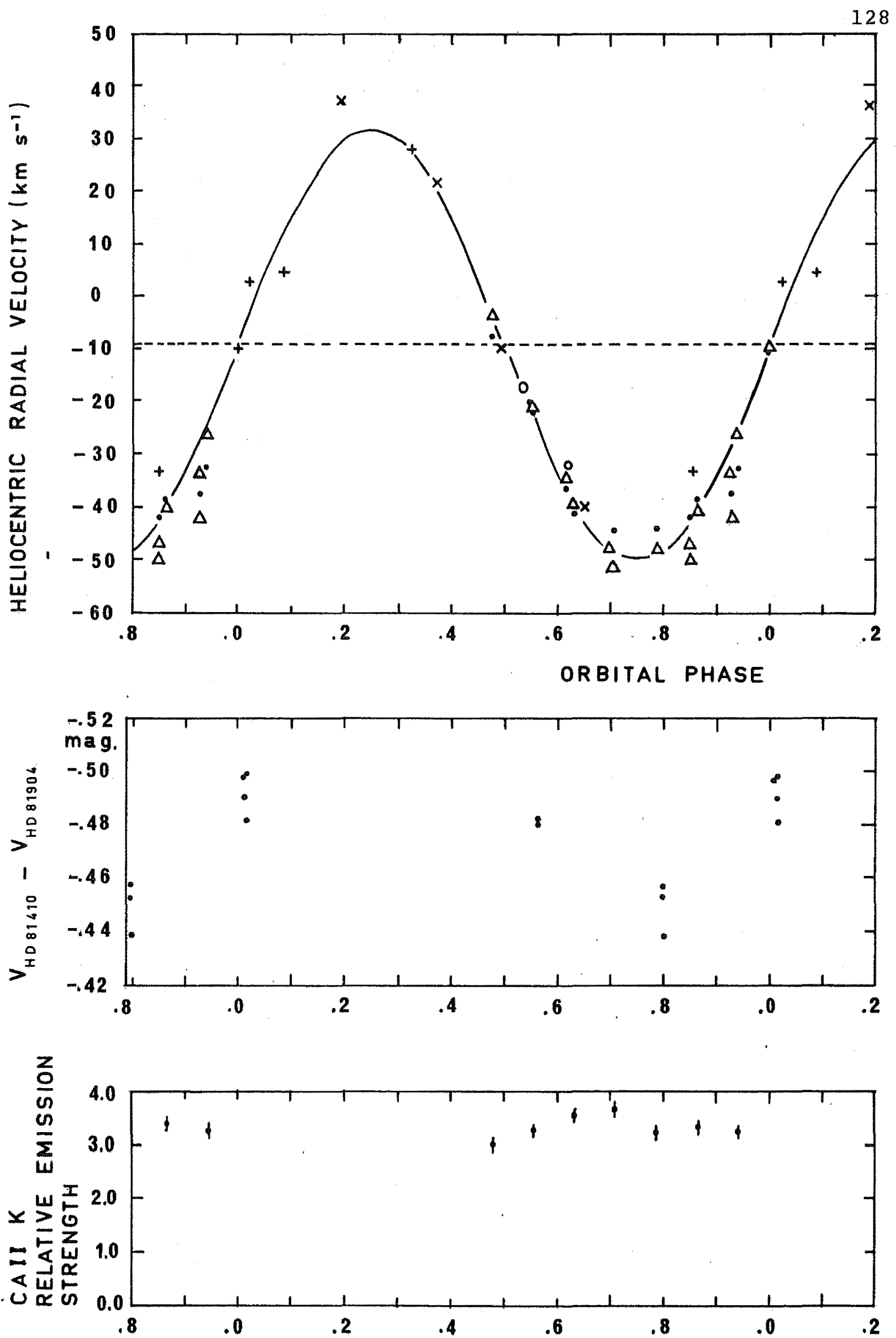

Fig. 4.12.3(a) Radial velocities of HD 81410 . Symbols defined as in Fig. 4 11. 3.

(b) V lightcurve of HD 81410, 1981 March-May.

(c) Ca II K emission flux variations in HD 81410, 1981 Apri1 13-20. 20 random error bars included. 
The radial velocities are summarised in Table 4.12 .1 , and the periodogram obtained using Gieseking's method is illustrated in Fig. 4.11.2. Two possible periods are present in the data, at $\mathrm{P}=12.9$ and $\mathrm{P}=25.4$ days. The latter solution could not, however, be reconciled with the data of Wayman and Jones (Eggen, 1973), so the former solution was adopted. The data of Wayman and Jones were incorporated in the solution, and $a$ period $\mathrm{P}=12.9054 \pm 0.0002$ days was found. The radial velocity solution (assuming a circular orbit) is given in Table 3.6 , and the radial velocity variation is plotted modulo orbital phase in Fig. $4.12 .3(a)$, along with the least-squares solution.

Although the Ca II K observations of 1981 April do not cover a complete orbit, a smooth variation in the emission flux was seen, which appeared to attain a maximum value close to the phase of photometric wave minimum (Fig. 4.12.3(b) and (c)).

The Wilson-Bapp $\mathrm{u}$ width of the Ca II $\mathrm{K}$ emission core yields a surface gravity $\log g=3.3 \pm 0.5$ (Section 3.5.2). The value obtained for the absolute surface flux in the Ca II $\mathrm{H}$ and $\mathrm{K}$ emission cores is $\mathrm{F}\left(\mathrm{K}_{1}\right)+\mathrm{F}\left(\mathrm{H}_{1}\right)=4.8 \times 10^{6}$ erg $\mathrm{cm}^{-2} \mathrm{~s}^{-1}$, and is probably uncertain by about $20 \%$, depending on systematic errors in the flux calibration and the intrinsic variability of the emission flux.

\subsubsection{Photometric Observations}

Photometric observations made at MJUO during 1980 May and at MSO during 1981 March-May yielded colour indices as listed in Table 2.3 , in good agreement with the UBV data of Eggen and with Wayman's spectral type for HD 81410. The 1981 March-May V lightcurve is plotted modulo orbital phase 

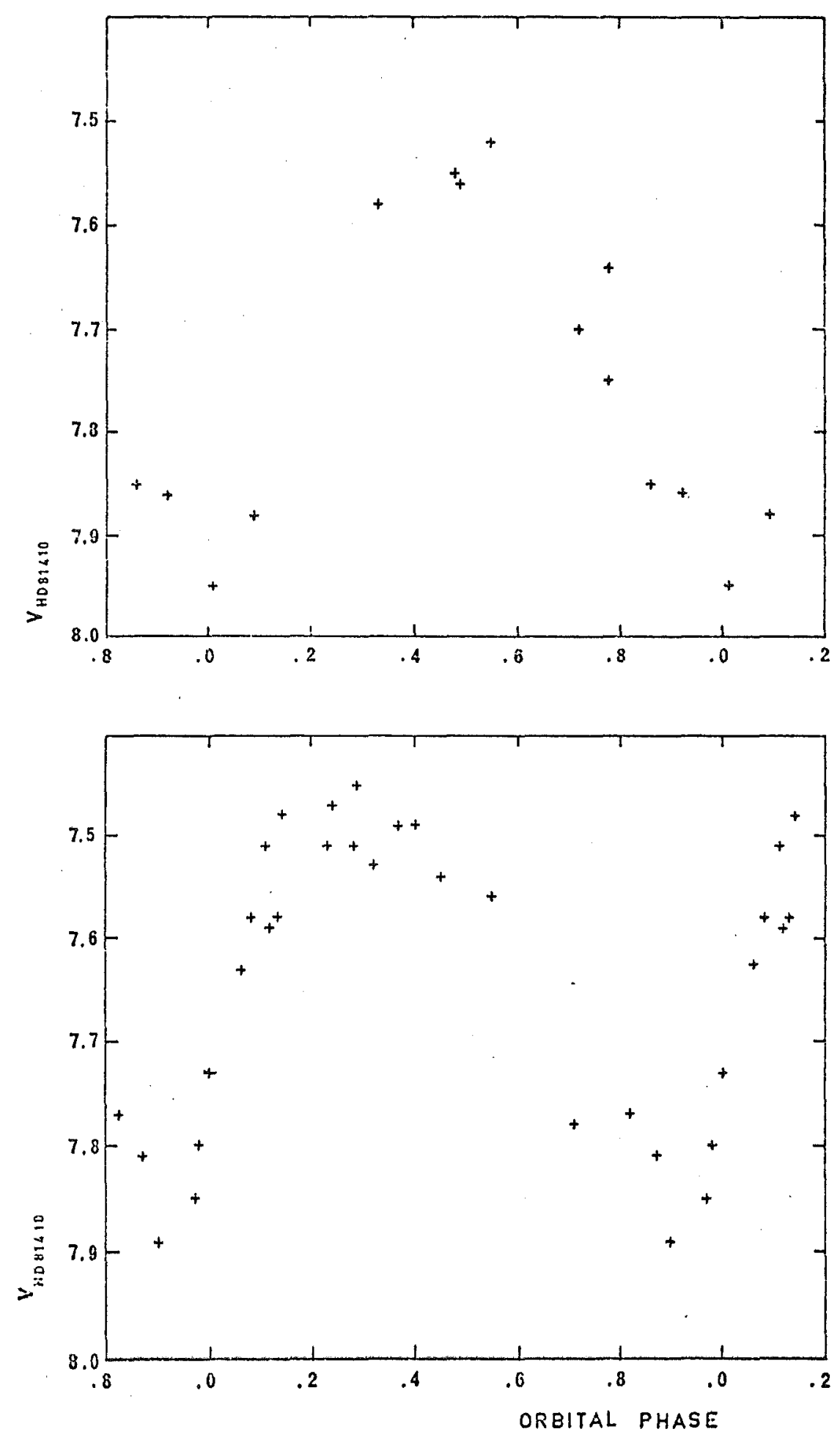

Fig. 4.12.4 V lightcurve of HD 81410 from photometric data by $0 . J$. Eggen (1973) during the observing seasons of (a) 1971 and (b) 1972 . 
in Fig. 4.12.2 (b).

The lightcurve coverage is sparse; however, a complete lightcurve of this star has been published by Raveendran, Mekkaden and Mohin (1981). They find that the variations have a 12.9 day periodicity, in good agreement with the spectroscopic orbital period. The lightcurve is complex, with two minima at spectroscopic orbital phases 0.45 and 0.85 . No evidence for eclipses is seen.

A rather greater wave amplitude is present in Eggen's 1971 and $1972 \mathrm{~V}$ data, plotted in Fig. 4.12.4 using the same ephemeris (based on the radial velocity data discussed in Section 4.12.2). Clearly, Eggen's data is consistent with the shorter period, showing a smooth variation with orbital phase. The lightcurves show little change in amplitude during 1971 and 1972, but a slow drift in the phase of wave minimum towards decreasing orbital phase is evident. The wave amplitude appears to be about 0.35 magnitude in both sets of Eggen's data, and the $V$ magnitude near wave maximum is about 7.5. The $\mathrm{V}$ magnitude at wave maximum during 1981 was about 7.1 , and the amplitude was about 0.15 magnitude. It appears that the level of starspot activity on HD 81410 declined considerably during the decade from 1971 to 1981, and it seems likely that a spot cycle of the type proposed by Shore and Hall (1980) may be operating in this star.

\subsubsection{Discussion}

Hejlesen $(1980 \mathrm{a}, \mathrm{b})$ has published evolutionary trades in the $\left(\log 9, \log \mathrm{T}_{e}\right)$ plane for stars of various masses and compositions. The Wilson-Bappu surface gravity and the effective temperature (estimated from the broad-band 
colour indices and the relations of Johnson (1966)) place HD 81410 near the base of the giant branch for stars of between 0.8 and 2.0 solar masses.

The observed rotational smearing of the spectrum, $v_{e} \sin i=15 \pm 5 \mathrm{~km} \mathrm{~s}^{-1}$ yields a minimum radius (for $i=90^{\circ}$ ) of $3.5 \pm 1.2 R_{\odot}$ for the active star. The actual radius is unlikely to be more than about twice this value. The high amplitude of the photometric variation observed by Eggen (1973) indicates that the star is viewed nearly equator-on.

Although nothing more definite can be said about the dimensions of the active star, they are clearly in the same range as those determined by other, more accurate means for the double-lined and eclipsing RS CVn binaries.

The secondary is probably a main-sequence star. The lack of an observable secondary spectrum at either $\mathrm{H} \alpha$ or the $H$ and $K$ lines provides evidence that the mass ratio $\mathrm{q}=\frac{\mathrm{m}_{2}}{\mathrm{~m}_{1}}$ is less than about 0.8 . The mass function $f(m)=(9.0 \pm 0.7) \times 10^{-2} M_{\odot}$ was measured from the radial velocity curve. The mass function may be written as

$$
f(m)=\frac{m_{1} q^{3} \sin ^{3} i}{(1+q)^{2}}
$$

For $q=0.8$, and considering the range of masses derived from comparison with Hejlesen's evolutionary tracks, this gives a minimum orbital inclination $i=36^{\circ}$, in. agreement with the earlier conclusion based on the amplitude of the photometric wave. 
$4.13 \quad \mathrm{HD} 101309$

HD 101309 appears on Bidelman and MacConnell's (1973) list of southern Ca II emitters, with a spectral classification of KO III/IV. Several low-resolution blue plates taken by Hearnshaw (1979) at MJUO show single emission cores in the $H$ and $\mathrm{K}$ lines, of comparable strength (with respect to the surrounding continuum) to those in HR 1099.

\section{$\underline{4.13 .1 \quad \mathrm{H}_{\alpha} \quad \text { Spectra }}$}

Two coudé êchelle spectra of the Ha region in HD 101309, obtained at MSO on 1981 February 22 and July 19, show Ha as an emission feature of variable strength (Fig. 4.13.1). The surrounding metallic line spectrum is rotationally broadened, with $v_{e} \sin i=15 \pm 5 \mathrm{~km} \mathrm{~s}^{-1}$. In both spectra, an $\mathrm{H} \alpha$ absorption feature due to the secondary component is visible; however, the metallic line spectrum of the secondary is lost in the noise in both spectra. The depth of the $\mathrm{H} \alpha$ absorption suggests that the secondary contributes about $25 \%$ of the total flux at this wàvelength.

This gives a magnitude difference of $1.2 \pm 0.3 \mathrm{mag}$. in a narrow band centred at $\sim 6560 \AA$. The emission feature is associated with the more Iuminous component at this wavelength; in the 1981 July 19 spectrum, the emission strength is comparable to that seen in HR 1099 in its quiescent state.

The radial velocities of the metallic line spectrum are listed in Table 4.13 .1 
TABLE 4.13 .1

\begin{tabular}{|c|c|c|c|c|c|c|c|}
\hline Date & Exposure & HJD & Heli & Locentr & L Radial & Velocity & $\left(\mathrm{km} \mathrm{s}^{-1}\right)$ \\
\hline U.T. & (sec.) & $2444000+$ & $\mathrm{H} \alpha$ & & $20 \AA$ & $\mathrm{K}$ & $\mathrm{H}$ \\
\hline & & & A & $\mathrm{A}^{\mathrm{A}}$ & B. & $\begin{array}{c}\mathrm{Em} . \\
\mathrm{A}\end{array}$ & $\begin{array}{c}\text { Em. } \\
\text { A }\end{array}$ \\
\hline 1981 Feb.22 & 4030 & 658.18 & 46.1 & & & & \\
\hline 1981 Mar.20 & 3600 & 684.13 & & 27.9 & -8.4 & 27.3 & \\
\hline 1981 Mar.21 & " & 685.07 & & 7.11 & nerged) & 8.0 & \\
\hline 1981 Mar.22 & $"$ & 686.04 & & -3.31 & nerged) & -7.6 & \\
\hline $1981 \operatorname{Mar} .24$ & 2000 & 687.99 & & -34.4 & 46.0 & -33.9 & -39.4 \\
\hline 1981 Mar.25 & 3600 & 689.02 & & -29.5 & 42.4 & -28.3 & -42.9 \\
\hline 1981 Mar.26 & 2410 & 689.96 & & 21.61 & herged) & -14.8 & -17.6 \\
\hline 1981 Apr.13 & 2512 & 707.98 & & 13.8 & -9.3 & 21.0 & \\
\hline 1981 Apr.14 & 3600 & 709.01 & & 4.01 & herged) & 3.4 & \\
\hline 1981 Apr.15 & " & 710.00 & & -13.7 & 36.4 & -12.9 & \\
\hline 1981 Apr.16 & $"$ & 710.99 & & -25.3 & 44.2 & -26.5 & \\
\hline 1981 Apr.17 & $"$ & 711.99 & & -25.2 & 47.1 & -20.7 & \\
\hline 1981 Apr.18 & $"$ & 712.98 & & -9.8 & 40.4 & -14.9 & \\
\hline 1981 Apr.19 & " & 713.97 & & 1.2 & 19.2 & 3.6 & \\
\hline 1981 Apr.20 & $"$ & 715.03 & & 3.91 & lerged) & 22.2 & \\
\hline 1981 Jul.19 & 4000 & 804.93 & -38.4 & & & & \\
\hline
\end{tabular}

Table 4.13. 1. Radial velocities of $\mathrm{HD} 101309 \mathrm{~A}$ and $\mathrm{B}$ from H $\alpha$ and Ca II spectra, MSO and SSO, 1981. 

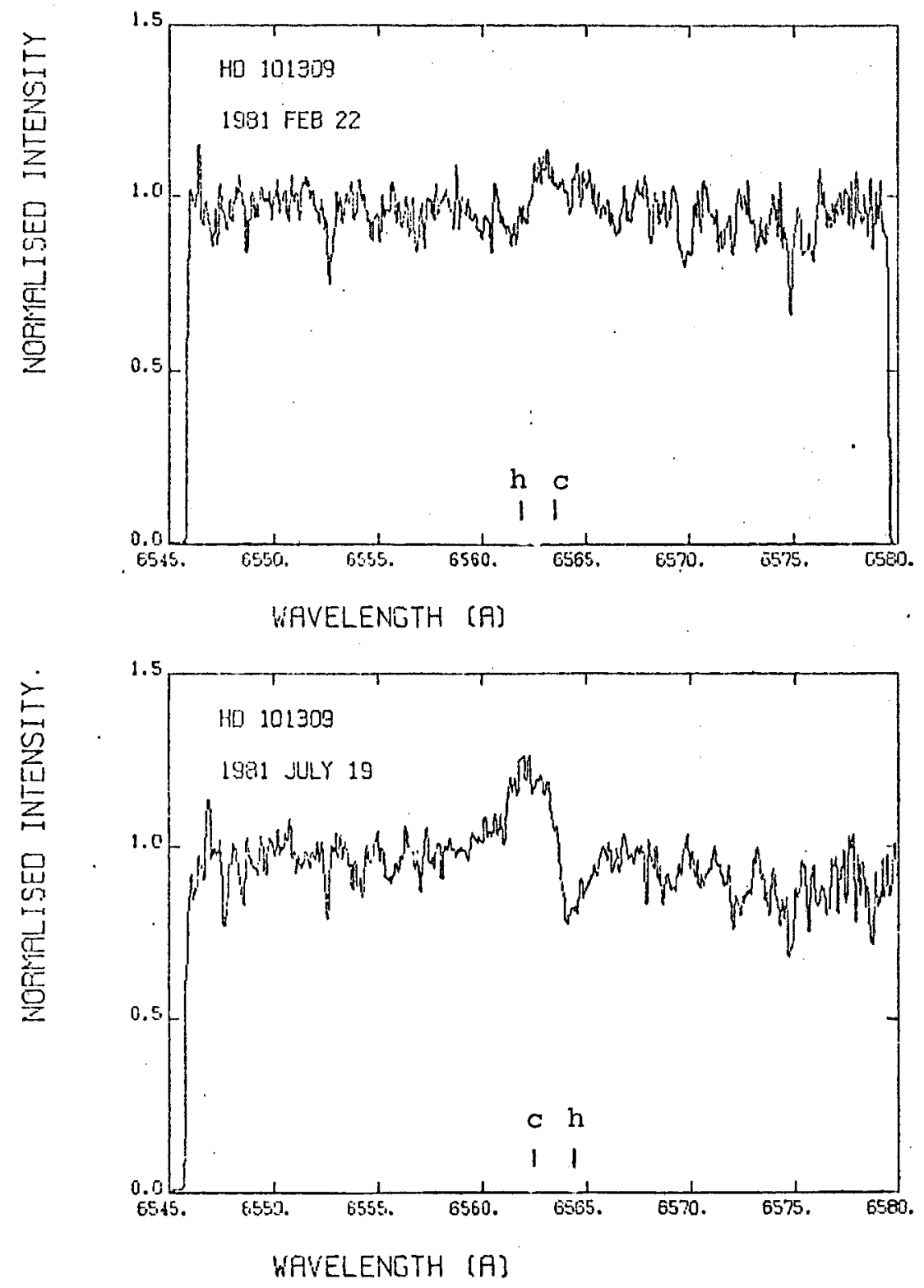

Fig. 4.13.1 H H spectra of HD 101309.

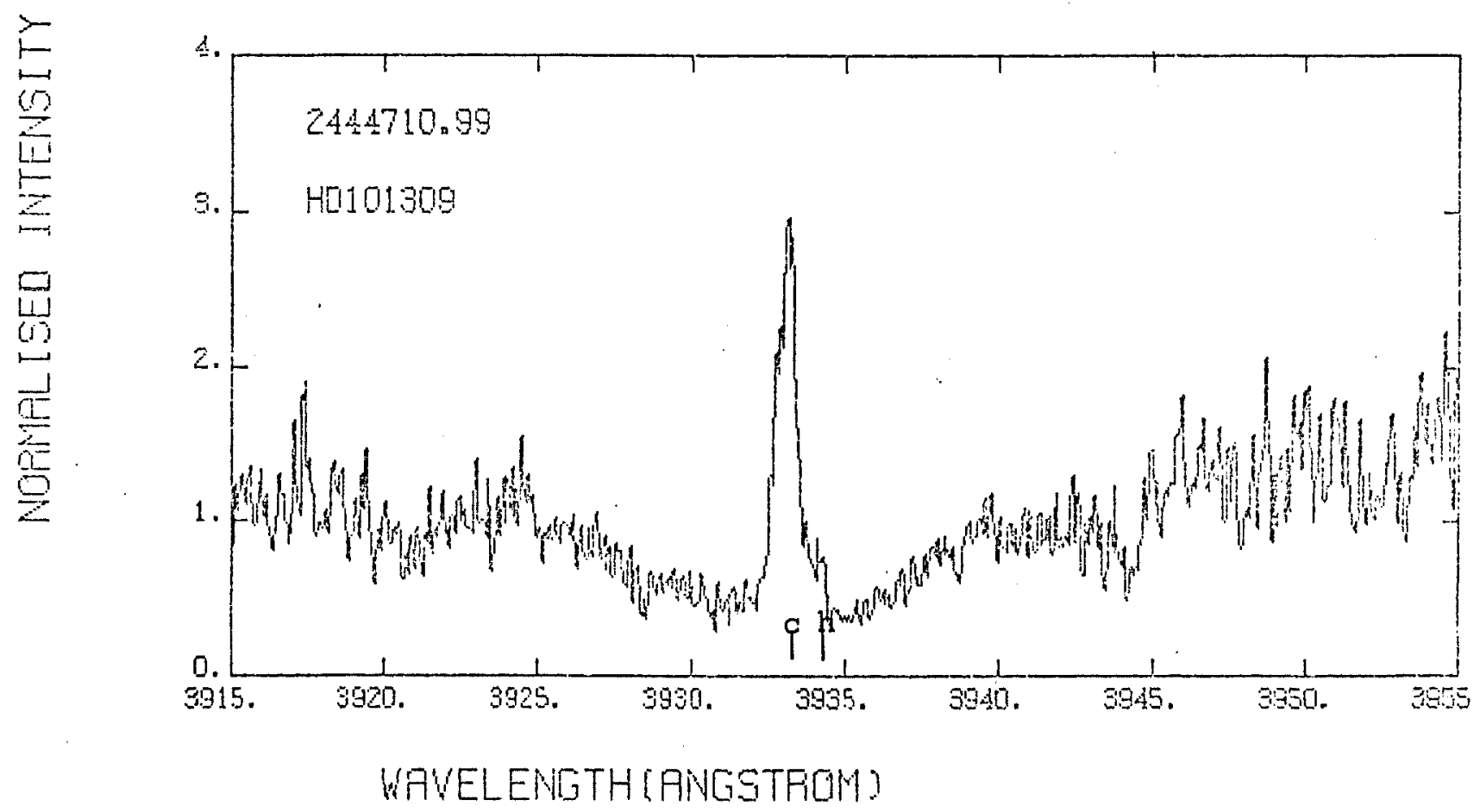

Fig. 4.13.2 Ca II K profile of HD 101309 at orbital phase 0.708 . Note weak emission feature due to secondary component. 


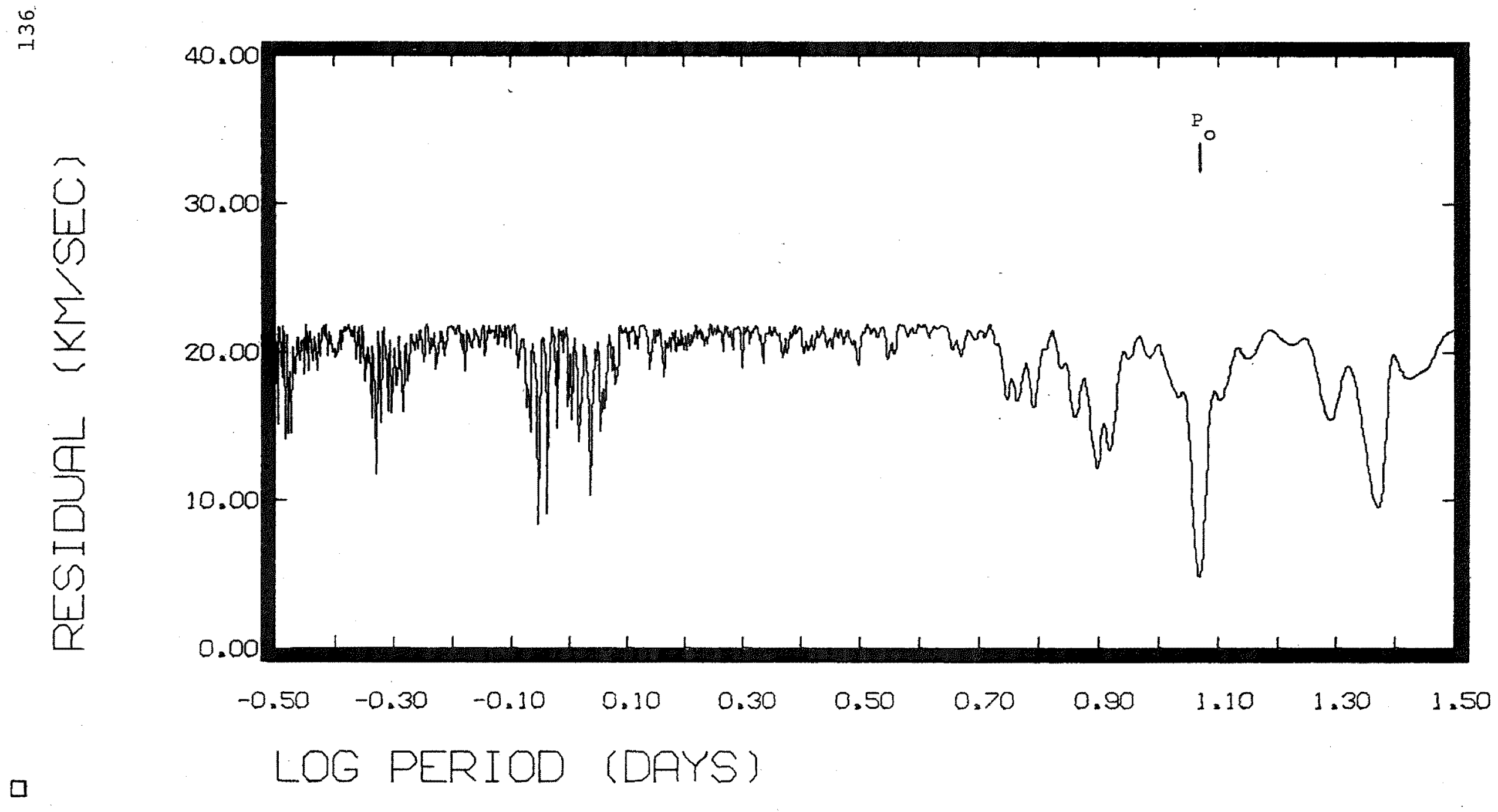

Fig. 4.13.3 Periodogram for HD 101309 radial velocities. 

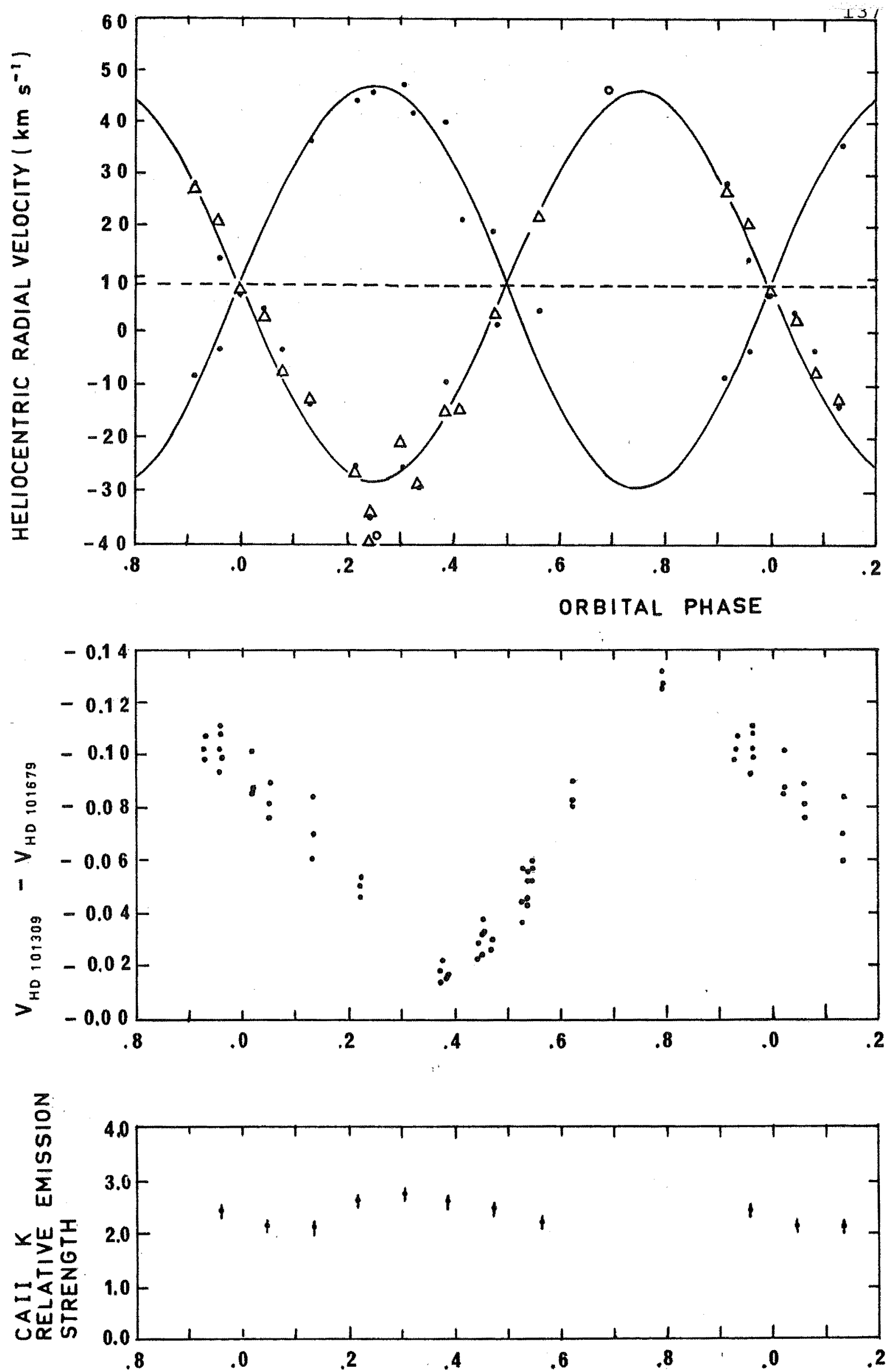

Fig. 4.13.4(a) Radial velocities of $\mathrm{HD} 101309$. Symbols defined as in Fig. 4.11. 3.

(b) V lightcurve of HD 101309, 1981 March-May.

(c) Ca II K emission flux variations in HD 101309, 1981 April 13-20. 20 xandom error bars included. 


\subsubsection{Ca II $\mathrm{H}$ and $\mathrm{K}$ spectra}

Ca II spectra obtained at MSO and sSo during 1981 March and April showed double absorption spectra of approximately equal strength in the region near the $H$ and $K$ lines. Near maximum velocity separation of the absorption spectra, a weak emission component was visible, (Fig. 4.13.2), sharing the radial velocity of one absorption spectrum, while the strong emission component's velocity variations matched those of the other spectrum. The radial velocities of the two components' emission and absorption spectra are listed in Table 4.13 .1 .

The method of Gieseking was applied to the radial velocities of the spectrum associated with the stronger emission component, and a period close to 11.7 days was found. The solution was extended to the dates of the $\mathrm{H} \alpha$ spectra, and the velocities of the strong Ca II emitting component and the component displaying the $\mathrm{H} \alpha$ emission were found to match.

The periodogram for the velocities of this star, including the velocities of the metallic line spectrum near $\mathrm{H} \alpha$, appears in Fig. 4.13.3. The radial velocity solution for an assumed circular orbit is given for this star (labelled component ' $A$ ') in Table 3.6. The velocity amplitude for component ' $B$ ' is also given in Table 3.6 , derived from a least-squares fit to the velocities of its spectrum using the ephemeris calculated for component ' $A$ '. Monitoring of emission flux variations in the Ca II $K$ line core (Section 3.5.6) was carried out during 1981 April 13-20 at sSO. The results, together with the radial velocity curve and the photometric V lightcurve, are plotted modulo orbital phase in Fig. 4.13.4. Despite the incomplete phase 
coverage, a smooth variation in the emission flux is seen, whose maximum coincides with the time of photometric wave minimum. The amplitude of the flux variation is enhanced by about $25 \%$, due to the depression of the continuum level at this wavelength near wave minimum. The variation is evidently real, and indicates that a localised enhancement of chromospheric heating is present in the vicinity of the spotted region on the surface of the active star. Similar correlations between the Ca II flux variations and the photometric wave were observed in HD 81410 (Section 4.12.2) and HD 127535 (Section 4.16 .2$)$.

\subsubsection{Photometric Observations}

Extensive UBV photometry of HD 101309 was obtained at MSO during 1981 March and April, concurrently with the Ca II spectroscopic program. A regular variation with a period of 11.7 days and an amplitude of 0.11 magnitude in $V$ was observed, with wave minimum occurring near orbital phase 0.4. The active star is clearly in synchronous rotation.

In order to estimate the effective temperatures and luminosities of the two components of HD 101309, we require information about the relative intensities of the two superimposed spectra at two or more widely-separated wavelengths.

The cross-correlation functions of the absorption spectrum in the region $3900-3930 \AA$ have twin peaks of approximately equal strength, indicating a luminosity ratio of about 1.0 at this wavelength. The magnitude difference between the two components at $\mathrm{H} \alpha$ was estimated as $1.2 \pm 0.3$ magnitudes in section 4.13.1. Making the rough assumption that the monochromatic magnitude difference will vary more or less linearly with wavelength for $3600 \AA \leqslant \lambda \leqslant 8100 \AA$, 
the above magnitude differences give $\Delta \mathrm{V}=0.7 \pm 0.2$

magnitude, and $\Delta\left(\mathrm{V}-\mathrm{R}_{\mathrm{J}}\right)=0.4 \pm 0.1$.

The ratio of the angular diameters of the two stars may be estimated from the empirical relations of Barnes, Evans and Moffett (1978)

$$
\log k=\log \frac{\phi^{\prime}}{\phi_{A}^{\prime}}=2\left[\mathrm{~F}_{\mathrm{A}}^{\mathrm{B}}-\mathrm{F}_{\mathrm{v}}^{\mathrm{A}}+0.1\left(\mathrm{~V}^{\mathrm{B}}-\mathrm{V}^{\mathrm{A}}\right)\right]
$$

where the $\phi^{\prime}$ are the angular diameters and the $F_{V}$ are the visual surface brightness parameters of the two stars, as defined by Barnes and Evans (1976). The $F_{V}$ are tabulated as a function of unreddened $\left(V-R_{J}\right)$ colour index by Barnes, Evans and Moffett (1978).

The luminosities and colour indices of the two stars must be such that their combined light will produce the observed colour indices of the system.

Using the information above in conjunction with the mean relations of Johnson (1966), models were constructed for a range of secondary spectral types.

The best agreement with the observed $(U-B)$ and $(B-V)$ colour indices was obtained for a model with a $68 \mathrm{~V}$ secondary $(B-V=0.74)$ and a KI IV primary $(B-V=1.06)$.

The calculated magnitude differences are $\Delta \mathrm{U}=-0.2$, $\Delta \mathrm{B}=+0.4, \Delta \mathrm{V}=+0.7, \Delta \mathrm{R}_{\mathrm{J}}=+1.0$, and $\Delta \mathrm{I}_{\mathrm{J}}=+1.2$, which are consistent with the observed flux ratios at $3920 \AA$ and $6560 \AA$.

The $\left(V-R_{J}\right)$ colour indices for this model are $0.60 \pm 0.02$ and $0.90 \pm 0.02$ for the hot and cool star respectively; the Barnes-Evans relation and Eq. 4.13-1 then give $k=0.35 \pm 0.04$. If we assume a minimum radius of $\sim 1 R_{\odot}$ for the $G 8 \mathrm{~V}$ secondary, the radius of the active component is at least $2.8 \pm 0.3 \mathrm{R}_{\odot}$. 


\section{$4.13 .4 \quad$ Discussion}

With the photometric model above in mind, an attempt may be made at calculating the absolute $\mathrm{Ca}$ II $\mathrm{H}$ and $\mathrm{K}$ surface emission flux for the active subgiant. The relative emission fluxes in columns 6 and 7 of Table 3.4 must be doubled, in order to compensate for the additional background flux near $3950 \AA$ due to the secondary's spectrum. The absolute fluxes in columns 8,9 and 10 were then calculated using the value $\left(V-R_{J}\right)=0.90 \pm 0.02$, as derived in section 4.13.3 above. The final result is $F\left(K_{1}\right)+F\left(H_{1}\right)=$

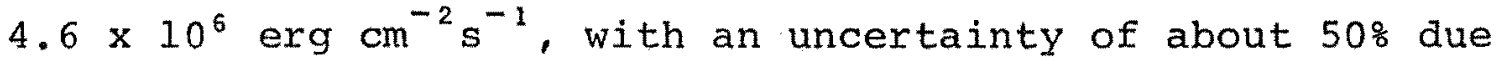
to intrinsic variability of the emission, systematic calibration errors and uncertainties in the assumed values for (a) the, secondary's contribution to the flux at $3950 \AA$, and (b) the $\left(V-R_{J}\right)$ colour index.

The surface gravity of the primary was determined from the Wilson-Bappu width of the Ca II $\mathrm{K}$ emission core (see Section 3.5.4), using an effective temperature of $4700 \pm 200 \mathrm{~K}$, appropriate to the photometric model. The result is $\log g=3.6 \pm 0.5$. Comparison with Hejlesen's (1980b) evolutionary tracks in the $\left(\log g, \log \mathrm{T}_{e}\right)$ plane indicates that the mass of the active star is between about 0.9 and 1.6 solar masses. As the secondary component has a G dwarf spectral type and the system mass ratio is close to unity, the mass of both stars is probably close to $1.0 \mathrm{M}_{\odot}$. This in turn yields an orbital inclination $i=41^{\circ} \pm 4$. The rotational smearing of the spectrum then gives a radius of $5.0 \pm 2.0 \mathrm{R}_{\odot}$ for the active component in reasonable agreement with the photometrically-determined value. 
HR 4492 is classified as a G5/8 III object with strong $\mathrm{H}$ and $\mathrm{K}$ emission and suspected photometric variability by Houk and Cowley (1975). The star forms a visual binary with the AO star HD 101380, with a separation of 0.22 arc seconds and a position angle which has increased from $119^{\circ} .3$ in 1930 to $216^{\circ} .2$ in 1976 (Worley, 1981). The cool star is some 0.8 magnitude brighter than the A0 star in the visual.

Stacy, Stencel and Weiler (1980) found some evidence for radial velocity variations in the cool star's absorption spectrum, based on $40 \AA \mathrm{mm}^{-1}$ blue spectra.

Garcia et.al.(1980) identified HR 4492 as the optical counterpart of the UHURU X-ray source $4 U$ 1137-65, after a precise position was obtained with the HEAO I scanning modulation collimator. HR 4492 was thus one of the first four stars with RS CVn characteristics to be detected as hard $(\geqslant 2 \mathrm{keV}) \mathrm{x}$-ray sources, with an average flux density of $5 \mu J_{Y}(2-6 \mathrm{keV})$ during the HEAO 1 observations.

Radio observations of HR 4492 at $5 \mathrm{GHz}$ were obtained with the $64 \mathrm{~m}$ radio telescope at Parkes, N.S.W. during 1981 July. The star was detected as a strong, flaring radio source, as reported by Collier et.al. (1982).

\subsubsection{Ho spectra}

(a) MJUO

HR 4492 was the only star on the source lists of strong southern Ca II emitters sufficiently bright for high-resolution $\mathrm{H} \alpha$ spectroscopy to be carried out at MJUO. Ten $2 \AA \mathrm{mm}^{-1}$ spectra of the $\mathrm{H} \alpha$ region were obtained using the échelle spectrograph at the cassegrain focus of the $0.61 \mathrm{~m}$ Boller and Chivens 
telescope at MJUO. A single-stage Varo image

intensifier was used in conjunction with $\mathrm{H}_{2}$-sensitised Kodak IIaD plates. Typical exposure times were about 90 minutes. Comparison spectra of a thorium-argon lamp were recorded alongside the stellar spectra. The plates were traced using the Joyce-Loebl Mk.III microdensitometer at the University of Canterbury. Radial velocity measures were obtained by measuring the line positions in both the comparison spectra and the stellar spectrum to an accuracy of about $1 \mu$ on the Gaertner spectro-comparator at the University of Canterbury. Geocentric radial velocities were then derived from a third-order polynomial fit to lines of known wavelength in the comparison spectrum. These radial velocities are listed in Table 4.14.1, and refer to the metallic line spectrum of the cool star.

The observational uncertainty in these measures was $\pm 3 \mathrm{~km} \mathrm{~s}^{-1}$. The data in Table 4.14 .1 show that the cool component exhibits radial velocity variations with a total amplitude of some $20 \mathrm{~km} \mathrm{~s}^{-1}$, and a total amplitude of at least $20 \mathrm{~km} \mathrm{~s}^{-1}$.

The microdensitometer tracings of the MJUO spectra revealed a shallow $\mathrm{H} \alpha$ absorption core, with emission features of variable strength displaced some 100 $\mathrm{km} \mathrm{s} \mathrm{s}^{-1}$ towards both the red and the blue.

(b) MSO

High resolution $\mathrm{H} \alpha$ spectra were obtained using the coudé échelle spectrograph on the $1.88 \mathrm{~m}$ telescope at MSo during 1981 February and July. 
These spectra showed a similar $H \alpha$ profile to the earlier MJUO plates, and the rotational smearing of the metallic line spectrum was measured as $v_{e} \sin i=10 \pm 2 \mathrm{~km} \mathrm{~s}^{-1}$. An enhancement of the $\mathrm{H} \alpha$ emission strength was noted on $1981 \mathrm{July} 18$, coinciding with a flare-like increase in the radio flux at $5 \mathrm{GHz}$; this event has been described more fully by Collier et.al. (1982).

\section{$\underline{4.14 .2 \mathrm{Ca} \text { II } \mathrm{H} \text { and } \mathrm{K} \text { spectra }}$}

Seven spectra of the $H$ and $K$ line region were obtained at MSO during 1981 March. The light of the visual A0 companion dominates these spectra, with the spectrum of the cool star superimposed (Fig. 4.14.1). The measured radial velocity variations of the $\mathrm{Ca}$ II $\mathrm{H}$ and $\mathrm{K}$ emission cores were found to match those of the cool star's absorption spectrum. The radial velocities obtained by cross-correlation are listed in Table 4.14.1.

A period analysis of the cool star's radial velocity variations was performed using the method of Gieseking (Section 3.7 ). The resulting periodogram is illustrated in Fig. 4.14.2, and gives a period of $53.85 \pm .01$ days. The full radial velocity solution is given in Table 3.6 , and the radial velocity data are plotted modulo orbital phase in Fig. 4.14 .3 (a).

The Wilson-Bappu width of the Ca II $\mathrm{K}$ emission core was measured as $W_{0}=1.3 \pm 0.2 \AA$, after corrections were made for the rotational smearing and instrumental resolution. 


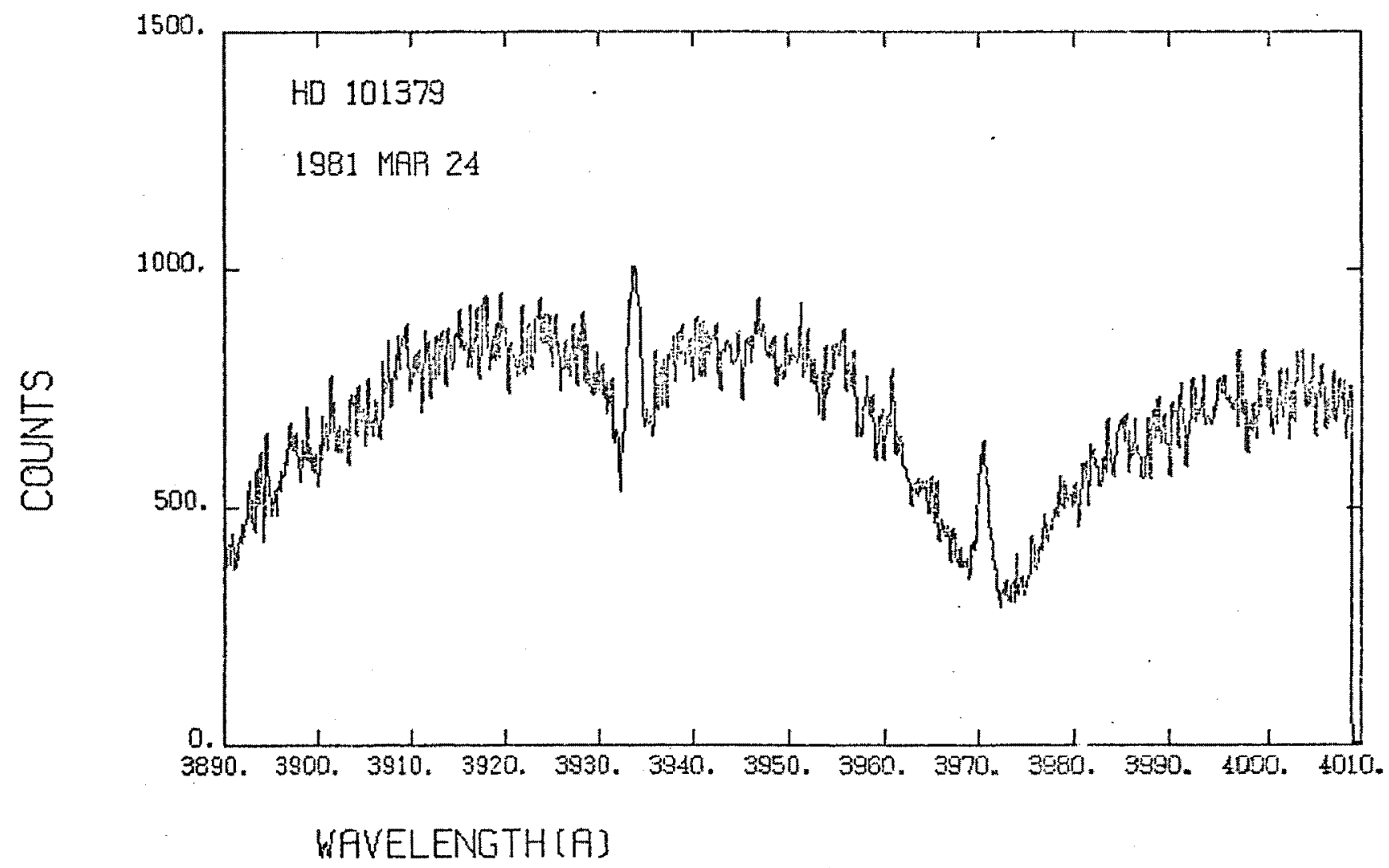

Fig. 4.14.1 Ca II $\mathrm{H}$ and $\mathrm{K}$ spectrum of HR 4492. 


\begin{tabular}{|c|c|c|c|c|}
\hline & & TABLE & $4.14 \cdot 1$ & \\
\hline $\begin{array}{l}\text { Date } \\
\text { U.T. }\end{array}$ & $\begin{array}{c}\text { Exposure } \\
\text { (sec.) }\end{array}$ & $\begin{array}{c}\text { HJD } \\
2440000+\end{array}$ & 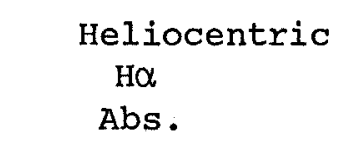 & $\begin{array}{l}\text { Radial Velocity }\left(\mathrm{km} \mathrm{s}^{-1}\right) \\
3920 \AA \\
\text { Abs. }\end{array}$ \\
\hline 1978 Feb. 27 & 6780 & 3567.04 & -4.2 & \\
\hline 1979 May 8 & 5400 & 4002.00 & -3.4 & \\
\hline 1980 Jun. 7 & 5880 & 4398.05 & 20.0 & \\
\hline 1980 Jun. 8 & 2400 & 4398.88 & 20.3 & \\
\hline$"$ & 5400 & 4399.04 & 20.8 & \\
\hline$"$ & $"$ & 4399.12 & 21.8 & \\
\hline 1980 Jun. 9 & $"$ & 4399.96 & 21.1 & \\
\hline 1980 Jun. 11 & $"$ & 4401.79 & $18-7$ & \\
\hline 1980 Jul. 29 & 6000 & 4449.94 & 15.5 & \\
\hline $1980 \mathrm{Jul} .30$ & 7200 & 4450.91 & 17.5 & \\
\hline 1981 Feb. 22 & 1150 & 4658.02 & 8.1 & \\
\hline 1981 Feb.23 & 980 & 4659.04 & 7.4 & \\
\hline$"$ & 1270 & 4659.20 & 7.8 & \\
\hline 1981 Mar. 24 & 1000 & 4688.01 & & 9.0 \\
\hline$"$ & $"$ & 4688.07 & & 11.6 \\
\hline$"$ & $"$ & 4688.13 & & 6.7 \\
\hline$"$ & $"$ & 4688.20 & & 7.5 \\
\hline$"$ & $"$ & 4688.24 & & 5.6 \\
\hline 1981 Mar. 25 & 3000 & 4689.06 & & 7.3 \\
\hline 1981 Mar. 26 & 1510 & 4689.98 & & 6.5 \\
\hline
\end{tabular}


TABLE 4.14 .1 continued

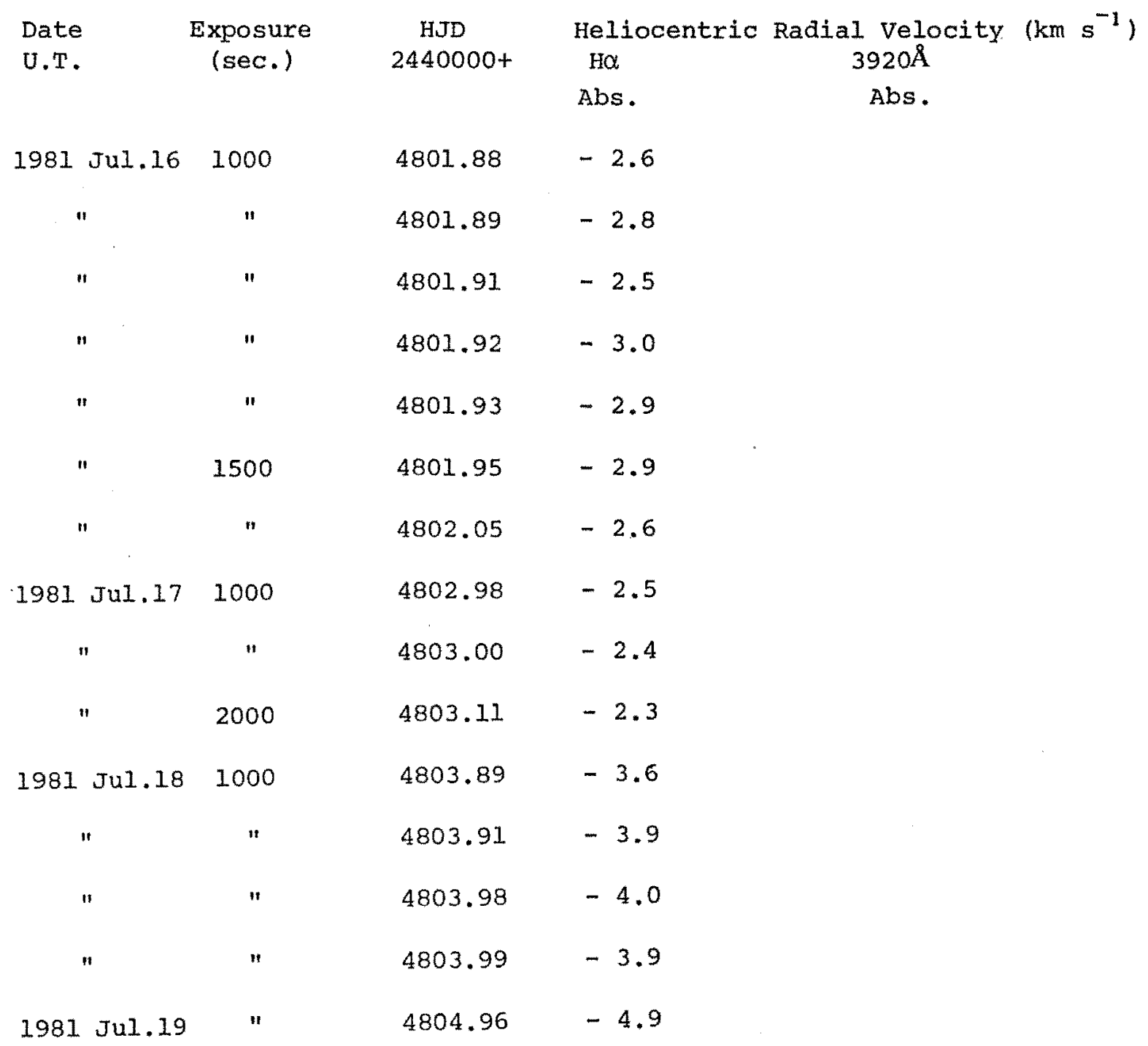

Table 4.14.1 Radial velocities of HR 4492 from $H$ and Ca II spectra, MJUO and MSO 1978-1981. 
$\stackrel{\infty}{\sim}$

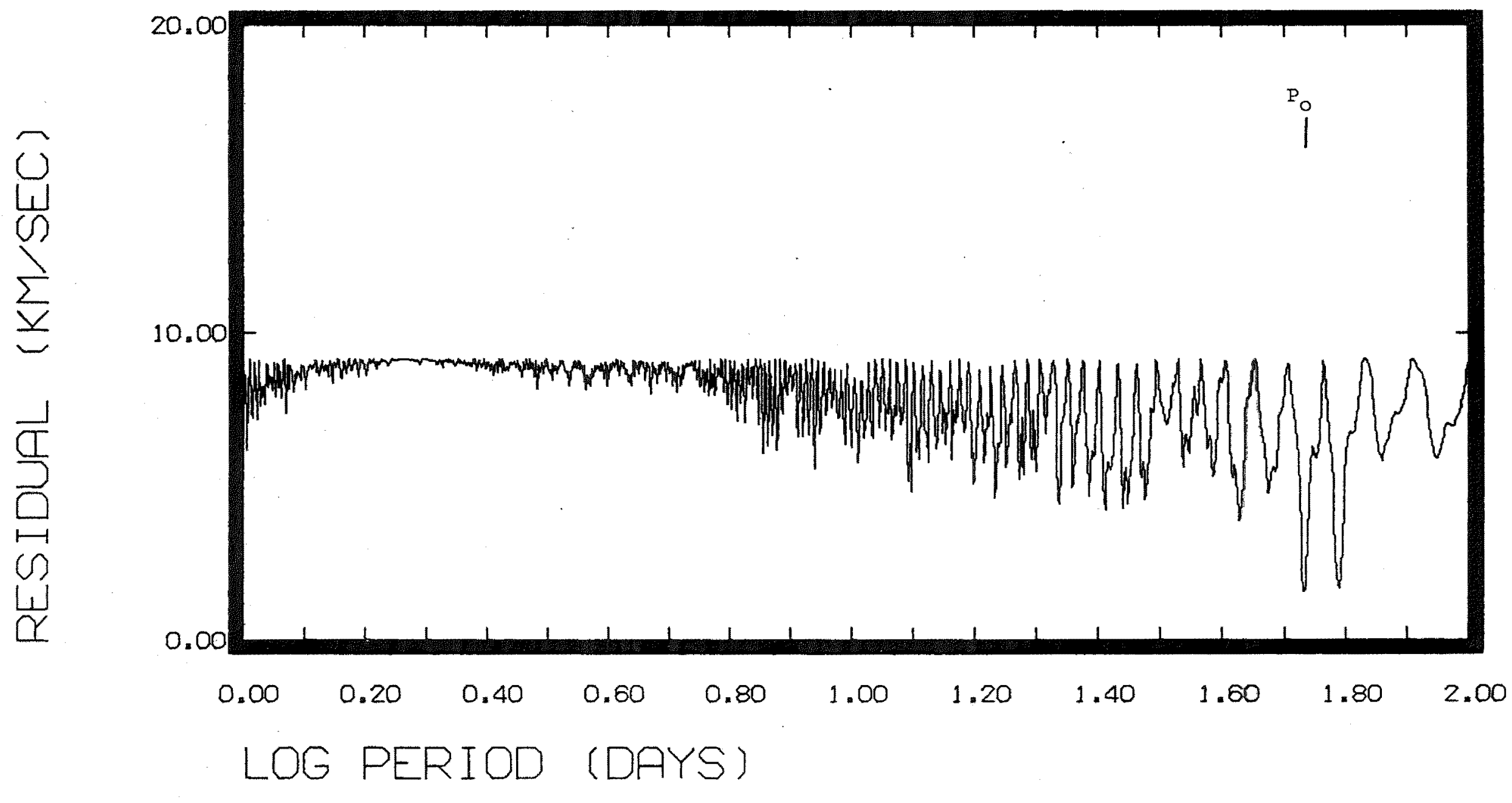

Fig. 4.14.2 Periodogram for HR 4492 radial velocities. 


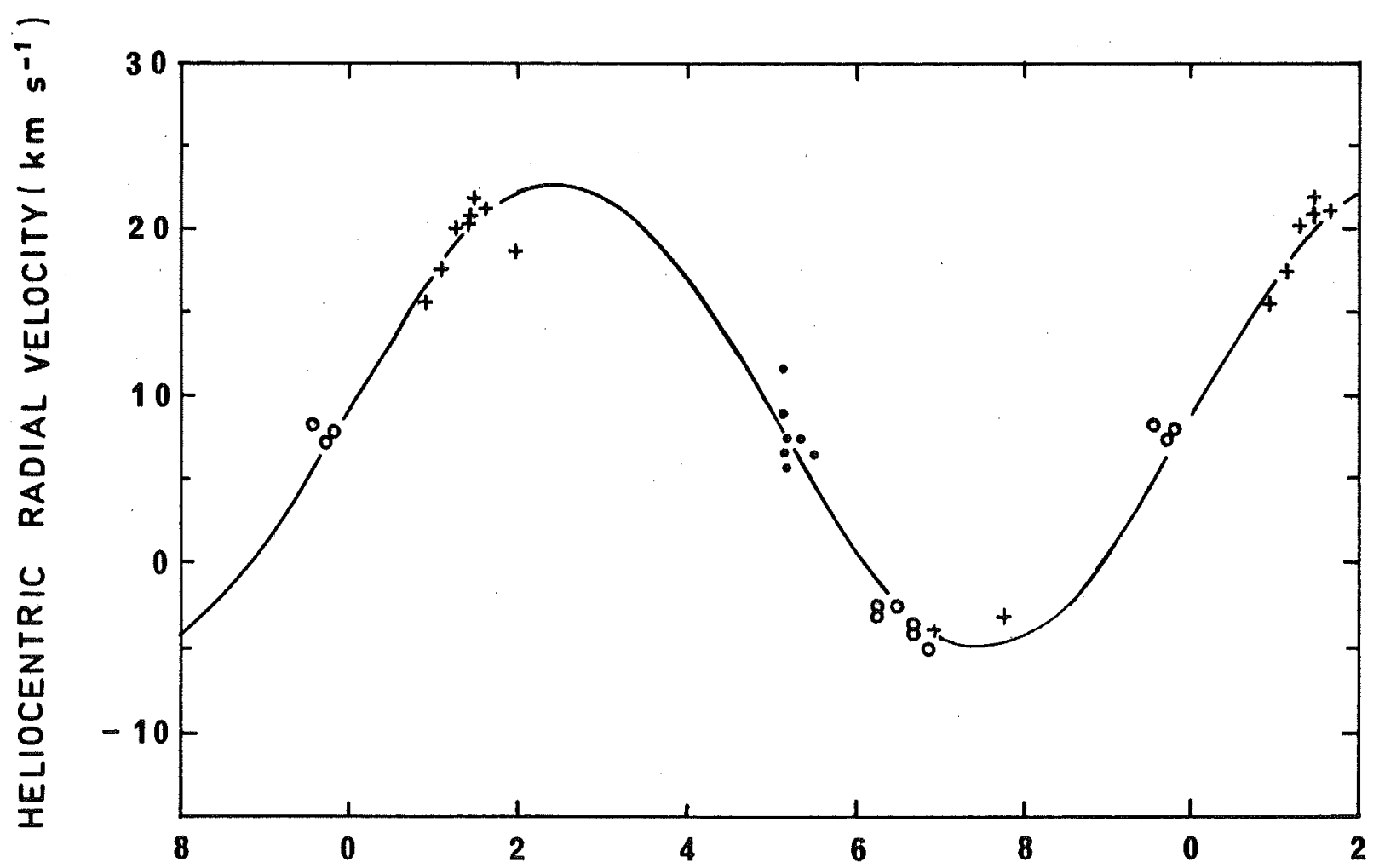

ORBITAL PHASE

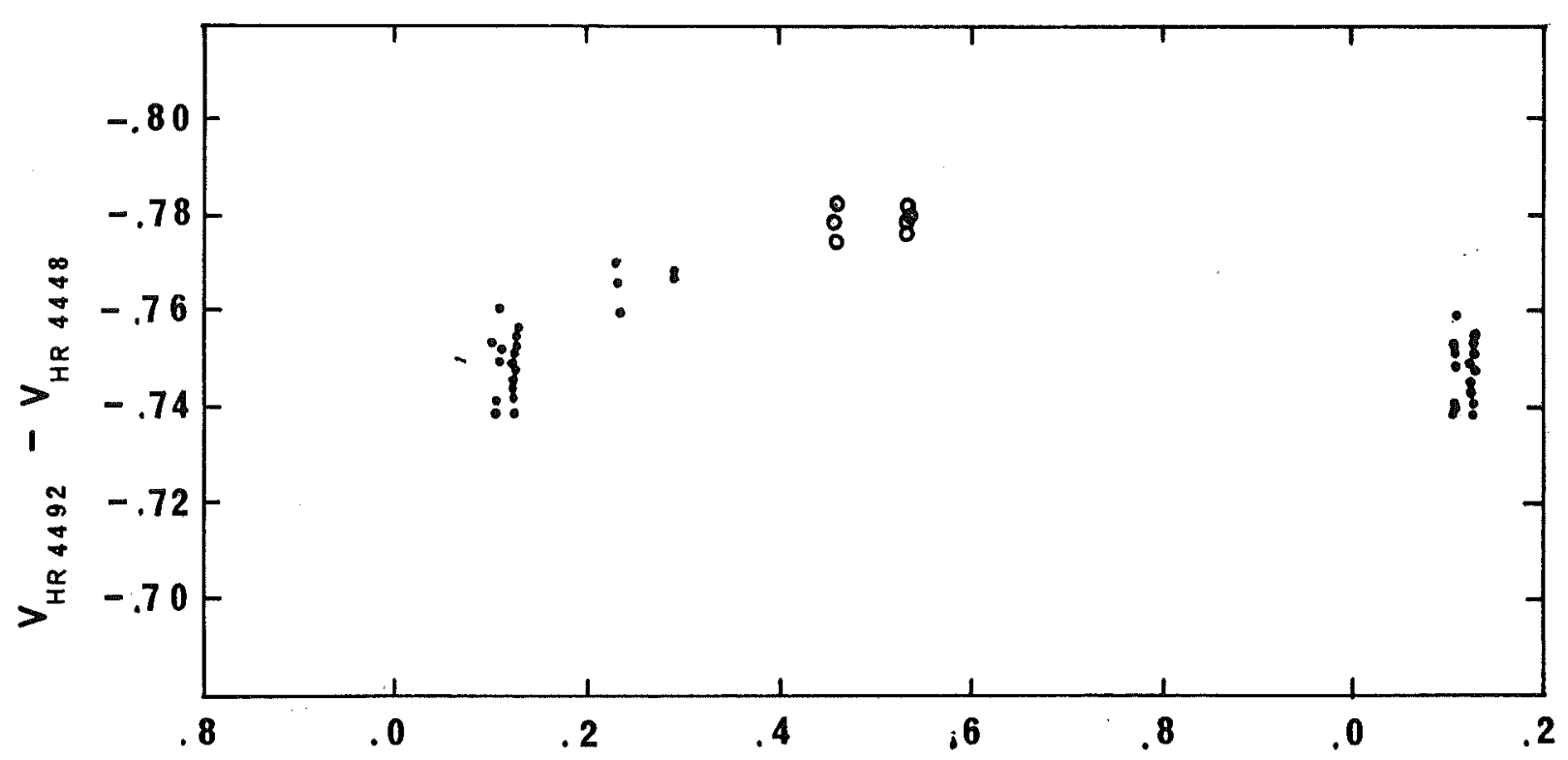

Fig. 4.14.3(a) Radial velocities of HR 4492. Crosses represent velocities from MJUo échelle spectra; symbols otherwise defined as in Fig. 4.11.3.

(b) V lightcurve of HR 4492, 1981 March-May. Eclipses of A system not included. The ephemeris used was HJD $=2444552.52+53.85 \mathrm{E}$. 
4.14.3 Photometric Observations and Analysis.

Photometric observations of HR 4492 were obtained during 1980 May at MJUO, and during 1981 March at MSO. The combined colours of the cool, active star and the A0 visual companion are listed in Table 2.6 .

Four shallow eclipses were observed. Three of. these, at HJD 2444364.090 (MJUO), 2444366.846 (MJUO) and 2444667.101 (MSO) had depths

$$
\begin{aligned}
\Delta \mathrm{V} & =0.068 \pm 0.005 \\
\Delta(\mathrm{U}-\mathrm{B}) & =0.114 \pm 0.010 \\
\Delta(\mathrm{B}-\mathrm{V}) & =0.070 \pm 0.005 \\
\Delta\left(\mathrm{V}-\mathrm{R}_{\mathrm{J}}\right) & =0.036 \pm 0.005 \\
\Delta\left(\mathrm{V}-\mathrm{I}_{\mathrm{J}}\right) & =0.005 \pm 0.005
\end{aligned}
$$

indicating that the eclipsed object had colour indices

$$
\begin{aligned}
& \mathrm{U}-\mathrm{B}=-0.02 \pm 0.08 \\
& \mathrm{~B}-\mathrm{V}=0.06 \pm 0.09 \\
& \mathrm{~V}-\mathrm{R}_{\mathrm{J}}=0.00 \pm 0.20 \\
& \mathrm{~V}-\mathrm{I}_{\mathrm{J}}=-0.34 \pm 0.40
\end{aligned}
$$

suggesting a spectral type close to A2 V (Johnson, 1966).

The fourth eclipse, at HJD 2444673.967, had depths

$$
\begin{aligned}
\Delta V & =0.078 \pm 0.005 \\
\Delta(\mathrm{U}-\mathrm{B}) & =0.164 \pm 0.005 \\
\Delta(\mathrm{B}-\mathrm{V}) & =0.088 \pm 0.005
\end{aligned}
$$

giving colour

indices for the eclipsed object

$$
\begin{array}{ll}
\mathrm{U}-\mathrm{B} & =-0.11 \pm 0.05 \\
\mathrm{~B}-\mathrm{V} & =0.01 \pm 0.05
\end{array}
$$

and hence a spectral type close to AOV (Johnson, 1966). If we label this star as the primary, the eclipse timings give an ephemeris for 
primary eclipses

$$
\begin{array}{r}
\text { HJD }=2444362.693 \pm 2.7546 \mathrm{E} \\
\pm .001 \pm 0.0004
\end{array}
$$

The eclipses clearly originate in the visual companion to the cool, active star. If we assume combined colour indices for the two stars in the eclipsing binary (hereinafter referred to as the "A system") similar to the mean values given by Johnson (1966) for an AI V star, the visual magnitude difference of 0.8 cited by Worley (1981) gives colour indices for the cool star

$$
\begin{aligned}
& \mathrm{U}-\mathrm{V}=2.4 \pm 0.1 \\
& \mathrm{~B}-\mathrm{V}=1.5 \pm 0.1 \\
& \mathrm{~V}-\mathrm{R}_{\mathrm{J}}=1.0 \pm 0.1 \\
& \mathrm{~V}-\mathrm{I}_{\mathrm{J}}=1.8 \pm 0.1
\end{aligned}
$$

which are consistent with a K4 III object with $\mathrm{T}_{\text {eff }}=$ $4000 \pm 100 \mathrm{~K}$

The radius of the cool star may be determined from the Barnes-Evans relation. The $V$ magnitude difference of 0.8 between the $\mathrm{A}$ system and the $\mathrm{K}$ giant, combined with the colour indices estimated above, gives

$$
\log \frac{R(K 4 \text { III })}{R(A \text { system })}=2\left(\Delta F_{V}+0.1 \Delta V_{o}\right)
$$

where the visual surface brightness parameter $F_{V}$ for each star is determined from the $(V-R)_{J}$ colour index using the calibration given by Barnes, Evans and Moffett.

The two stars in the $A$ system are likely to have radii of $1.7 \pm 0.3 \mathrm{R}_{\odot}$, assuming that they are typical main-sequence objects. If we treat them as a single object of radius $\sqrt{ } 2 \times(1.7 \pm 0.3) R_{0}$, we obtain

$$
\mathrm{R}(\mathrm{K} 4 \mathrm{III})=25 \pm 11 \mathrm{R}_{\odot} .
$$


The Wilson-Bappu width of the Ca II $K$ emission core is $W_{0}=1.3 \pm 0.2 \AA$ which, with $\mathrm{T}_{\mathrm{e}}=4000 \pm 100 \mathrm{~K}$, gives $\log g=1.4 \pm 0.7$

Comparison with the evolutionary models of Iben in the $\left(\log g, \log \mathrm{T}_{e}\right)$ plane suggests that the $\mathrm{K}$ giant has a mass between 2.5 and $5.0 \mathrm{M}_{\odot}$, and has reached an evolutionary stage close to the tip of the ascending giant branch, and has a radius

$$
R=60 \pm 30 R_{\odot}
$$

The lower end of this range is consistent with the photometric estimate of the radius, and suggests that the mass lies in the range $3.0 \pm 0.5 \mathrm{M}_{\odot}$. A lower limit may be placed on the mass, when we consider that the stars in the A system, which are physically associated and presumably of similar age, are still on the main sequence.

The V magnitude of HR 4492 was observed to vary slowly outside eclipse. A much smaller variation in the $B$ band was also noted, but no measurable variability was present in the $U$ band. The variation is evidently associated with the cool star, and in view of the other strong RS CVn characteristics of this object, is interpreted as being an RS CVn-type spot wave.

Unfortunately, the photometric observations cover only a narrow range of phases in the $\mathrm{K}$ star's spectroscopic 54-day binary orbit. However, the 1981 March and May MSO observations span two consecutive orbits and when plotted modulo orbital phase (Fig. 4.14 .3 (b)) the results are not inconsistent with synchronous rotation of the giant. Clearly, further photometric monitoring over an extended period is required to confirm that the slow photometric variation has the same period as the 54-day orbit; the presence of measurable 
rotational broadening in the giant's spectrum provides strong evidence that at least partially synchronous rotation has been achieved.

Bearing this in mind, we may attempt to calculate the inclination of the K star's orbit to the line of sight. In Table 4.14 .2 the value of the mass ratio $q$ is calculated, using Eq. 4.12-1, for various orbital inclinations.

A primary mass of $3.0 \mathrm{M}_{0}$ is assumed. The value of $\tau_{\text {sync }}($ column 4) is calculated using Zahn's (1977) approximate expression for the tidal synchronisation timescale of a binary component with a convective envelope. The Roche lobe radius is calculated using Paczynski's (1971) approximation for intermediate mass ratios $(0.05 \leqslant q \leqslant 3.0 ; \mathrm{Eq} .5-4)$.

The Roche radius derived in this way for the giant varies little with orbital inclination, and provides an upper limit on the radius calculated earlier. As this limiting value lies close to the upper limit for the photometric model and the low mass, high log $g$ limit for the evolutionary radius, it appears that the giant is very close to filling, if not overflowing, its Roche lobe.

Also, the synchronisation timescales calculated for a stellar radius of $30 \mathrm{R}_{\odot}$ are several orders of magnitude shorter than the thermal expansion timescale of the convective envelope $\left(\approx 10^{7}\right.$ years for a star with $\left.M \simeq 3 M_{0}\right)$.

It thus seems highly likely that the giant in the HR 4492 system is filling its Roche lobe and co-rotating with its companion. The observed rotational smearing of the $\mathrm{K}$ spectrum is $\mathrm{v}_{\mathrm{e}} \sin =10 \pm 2 \mathrm{~km} \mathrm{~s}^{-1}$, which under the above conditions yields an orbital inclination

$$
i=17^{\circ} \pm 4^{\circ}
$$


Such an inclination would imply a mass ratio which is remarkably close to unity. It seems unlikely that the companion contributes significantly to the overall system Iuminosity, in view of the close fit to the observed colour indices obtained by considering the light of the $K$ giant and the A system alone. This problem can only be resolved with further detailed photometric and spectroscopic observations. A detailed analysis of the A system's lightcurve must be made, in order to determine more precisely the physical dimensions of these two stars. High resolution spectroscopy of intermediate $(4000-5000 \AA)$ wavelengths with high signal-to-noise ratios should be carried out in an attempt to detect the spectrum of the $\mathrm{K}$ giant's companion. Thirdly, further photometry covering all phases of the $K$ star's orbit may give some insight into whether or not mass transfer is occurring in the 54-day binary system.

\subsubsection{Ca II Absolute Surface Flux Calibration}

Estimation of the Ca II surface flux in the $\mathrm{K}$ giant is complicated by the difficulty experienced in estimating the relative contributions of the $\mathrm{A}$ system and the $\mathrm{K}$ giant to the total flux in the band 3925-3975 $\AA$. Using the ratio of the angular diameters calculated earlier and the LWMR calibration (Section 3.5.5) for the flux in this band, we obtain

$$
\frac{F(\Delta \lambda)_{\mathrm{A}}}{F(\Delta \lambda)_{\mathrm{K}}} \cdot\left(\frac{\phi \mathrm{A}}{\phi_{\mathrm{K}}}\right)=8.0 \pm 4.0
$$

which is in reasonable agreement with the apparent ratio obtained by inspection of the spectra (Fig. 4.14.1). In order to correct for this, the relative fluxes listed in columns 6 and 7 of Table 3.4 were scaled up by a factor 
In

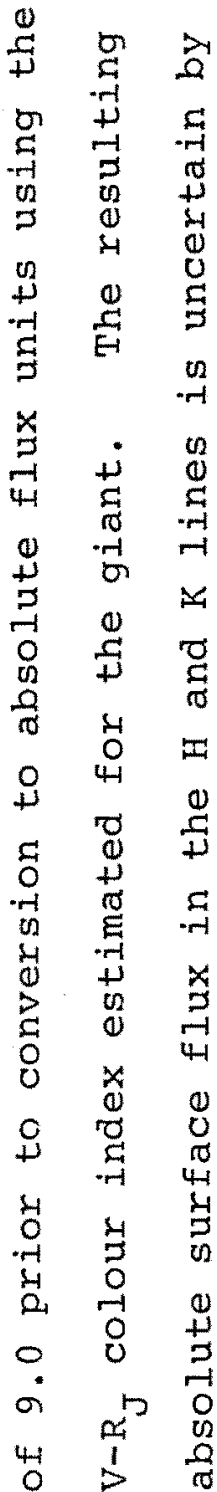

TABLE 4.14 .2

\begin{tabular}{|c|c|c|c|c|c|c|c|}
\hline $\begin{array}{l}\text { Primary } \\
\text { Mass } \\
\mathrm{m}_{1} / \mathrm{M}_{\odot}\end{array}$ & $\begin{array}{l}\text { Orbita } \\
\text { inclinat } \\
i\end{array}$ & tion & $\frac{f(m)}{m_{1} \sin ^{3} i}$ & $\begin{array}{l}\text { Mass } \\
\text { Ratio } \\
q=\frac{m_{2}}{m_{1}}\end{array}$ & $\begin{array}{l}\text { Synchronisation } \\
\text { timescale } \\
{ }_{\text {tsy no }} \text { (years) }\end{array}$ & $\begin{array}{l}\text { Orbital } \\
\text { Separation } \\
A / R_{\odot}\end{array}$ & $\begin{array}{l}\text { Roche } \\
\text { Lobe radius } \\
R_{L} / R_{\odot}\end{array}$ \\
\hline 3.0 & $90^{\circ}$ & & $5.00 \times 10^{-3}$ & 0.19 & $1.5 \times 10^{4}$ & 91.2 & 47.8 \\
\hline$"$ & $70^{\circ}$ & & $6.03 \times 10^{-3}$ & 0.21 & $1.3 \times 10^{4}$ & 91.5 & 47.3 \\
\hline$"$ & $50^{\circ}$ & & $1.11 \times 10^{-2}$ & 0.26 & $8.2 \times 10^{3}$ & 92.9 & 46.2 \\
\hline$"$ & $40^{\circ}$ & & $1.88 \times 10^{-2}$ & 0.36 & $4.4 \times 10^{3}$ & 86.8 & 40.7 \\
\hline$"$ & $30^{\circ}$ & & $4.00 \times 10^{-2}$ & 0.49 & $2.3 \times 10^{3}$ & 89.0 & 39.3 \\
\hline$"$ & $20^{\circ}$ & & $1.25 \times 10^{-1}$ & 0.83 & $8.2 \times 10^{2}$ & 94.8 & 37.6 \\
\hline$"$ & $15^{\circ}$ & & $2.88 \times 10^{-1}$ & 1.26 & $3.5 \times 10^{2}$ & 101.7 & 36.6 \\
\hline$"$ & $10^{\circ}$ & & $9.55 \times 10^{-1}$ & 2.54 & $8.7 \times 10^{1}$ & 117.9 & 35.2 \\
\hline 2.0 & $90^{\circ}$ & & $7.50 \times 10^{-3}$ & 0.22 & $5.0 \times 10^{3}$ & 80.3 & 40.9 \\
\hline$"$ & $10^{\circ}$ & & 1.43 & 2.69 & $3.5 \times 10^{1}$ & 116.1 & 34.1 \\
\hline Table & 4.14 .2 & \multicolumn{6}{|c|}{$\begin{array}{l}\text { Mass ratios, synchronisation } \\
\text { various primary masses and o } \\
\text { binary system. }\end{array}$} \\
\hline
\end{tabular}


HD 119285 is assigned a spectral classification of KI Vp by Houk and Cowley (1975) who also note the presence of strong $\mathrm{Ca}$ II $\mathrm{H}$ and $\mathrm{K}$ emission. Bidelman and MacConnell (1973) also classify this star as a Ca II emitter, but give the spectral type as K2 III/IV.

\subsubsection{Ha Spectra}

The Ha spectra of HD 119285 obtained at MSO during 1981 February and July, show a single, sharp, well-defined metallic line spectrum, with no measurable rotational smearing. The radial velocity is variable, with an amplitude of about $10 \mathrm{~km} \mathrm{~s}^{-1}$.

$\mathrm{H} \alpha$ itself appears as an emission feature of variable strength (Fig. 4.15.1), with a weak, narrow absorption core whose radial velocity matches that of the metallic line spectrum.

The radial velocities derived from the metallic Iines are listed in Table 4.15.1.

\section{$4.15 .2 \quad \mathrm{Ca}$ II $\mathrm{H}$ and $\mathrm{K}$ Spectra}

The cross-correlation functions derived for the absorption spectrum in the wavelength range $3900-3930 \AA$ show a single peak, whose radial velocities are listed in Table 4.15 .1 .

The eleven spectra of the $\mathrm{K}$ line region obtained at SSO during 1981 March and April showed little variation in the Ca II K emission core flux (Table 3.5 and Fig. 3.2). The Wilson-Bappu width of the $\mathrm{K}$ emission reversal, combined with an assumed effective temperature of $4600 \mathrm{~K}$ based on a $\mathrm{Kl} / 2 \mathrm{IV} / \mathrm{V}$ spectral type, gives a surface gravity $\log g=3.3 \pm 0.5$. Comparison with the evolutionary tracks 
of Hejlesen (1980b) yields a mass $\mathrm{M}=1.2 \pm 0.5 \mathrm{M}_{\odot}$ and radius $\mathrm{R}=4.0 \pm 2.0 \mathrm{R}_{\odot}$.

A period analysis of the radial velocity data using Gieseking's method (Fig. 4.15.2) gave an orbital period of 11.9 days. If synchronous rotation of the primary is assumed, the equatorial rotation speed will be $v_{e}=17 \pm 4$ $\mathrm{km} \mathrm{s} \mathrm{s}^{-1}$. The observed value of $\mathrm{v}_{e} \sin i$ is less than $\sim 8$ $\mathrm{km} \mathrm{s} \mathrm{s}^{-1}$, implying that the orbital inclination is probably less than $i=25^{\circ}$. The radial velocity curve is plotted in Fig.4.15.3.

The absence of an observable secondary spectrum indicates a magnitude difference $\Delta \mathrm{M}_{\mathrm{V}} \geqslant 1.5 \mathrm{mag}$. If the secondary is a main sequence object, its absolute visual magnitude must be $M_{v} \geqslant 5.2$, giving a maximum secondary mass of about $0.95 \mathrm{M}_{\odot} \cdot \quad$ The corresponding minimum orbital inclination (based on the radial velocity solution in Table $3.6)$ is $i=12^{\circ}$. Conversely, the maximum inclination $\left(i \approx 25^{\circ}\right.$ ) based on the rotational smearing, gives a minimum secondary mass of $0.4 \pm 0.1 \mathrm{M}_{\odot}$.

Although no photometry was obtained for HD 119285, the lack of any significant variation in the Ca II K emission flux lends support to models involving low values of the inclination, and secondary masses in the range $0.8 \leqslant \frac{M_{2}}{M_{O}} \leqslant 1.0$.

The value obtained for the $\mathrm{Ca}$ II $\mathrm{H}$ and $\mathrm{K}$ absolute surface flux (Table 3.4) is uncertain by about $50 \%$, as the lack of photometry of this star necessitated estimating $(V-R)_{J}$ from the spectral type. 

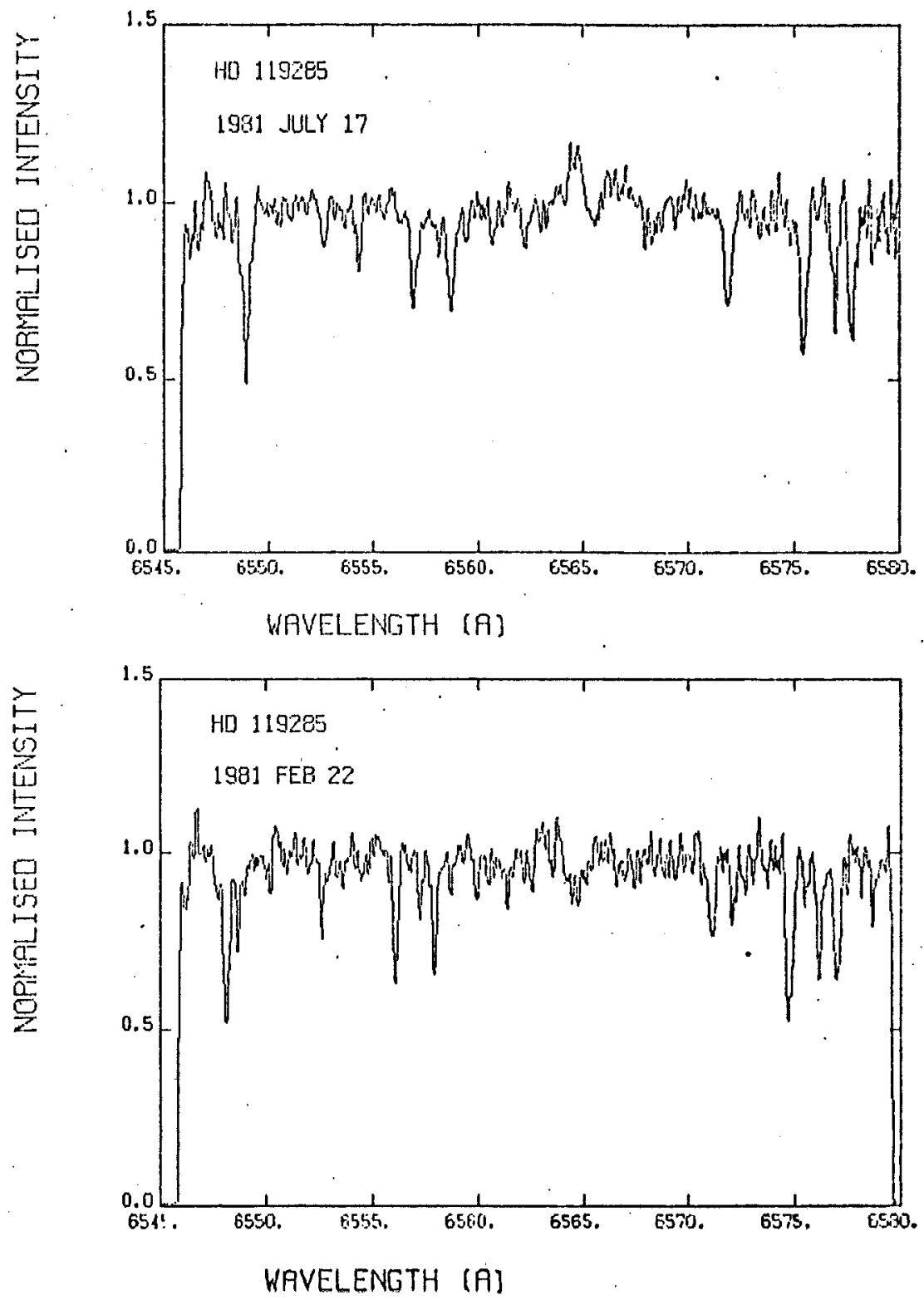

Fig. 4.15.1 H $\mathrm{H} \alpha$ spectra of HD 119285. 


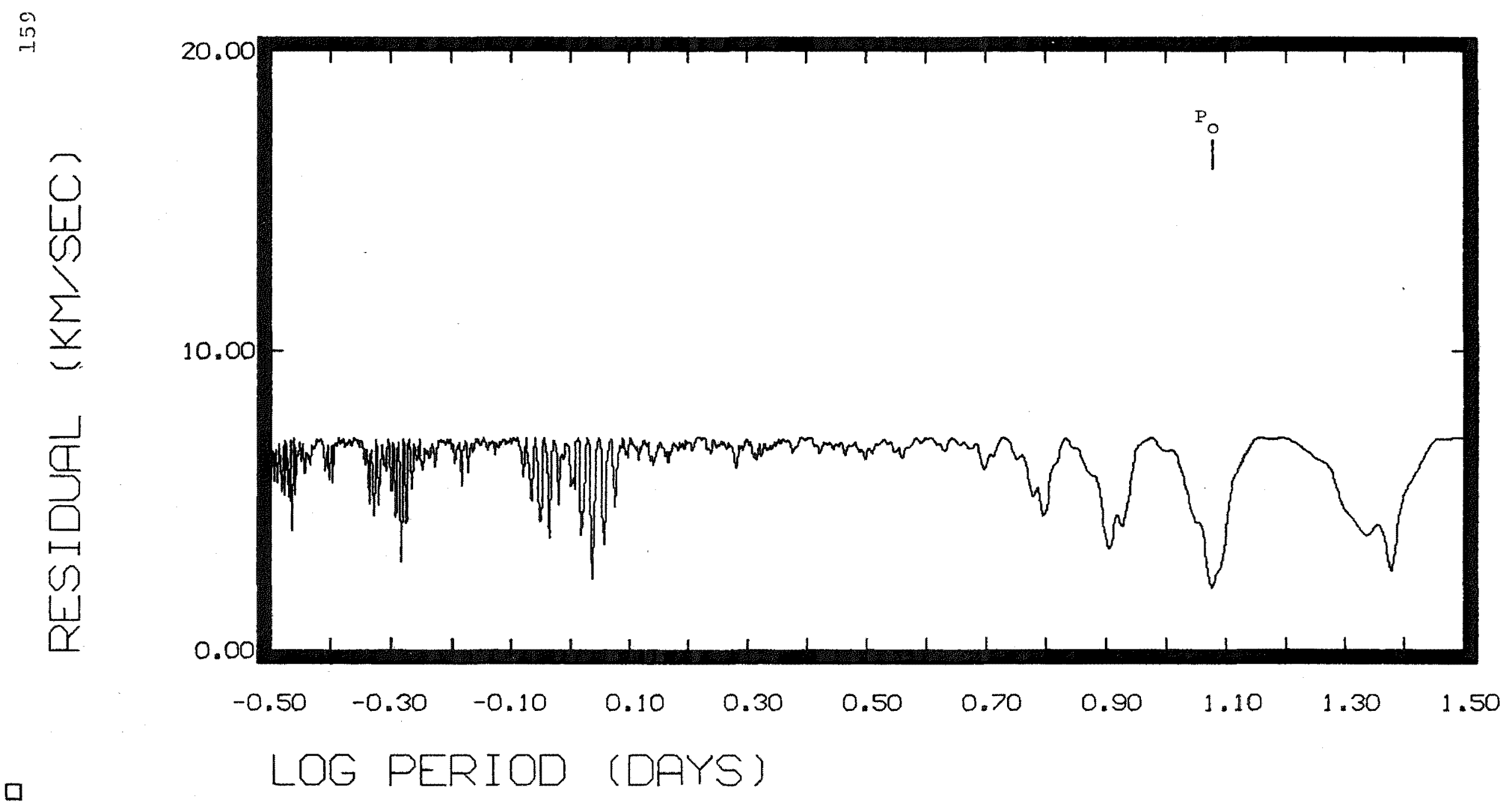

Fig. 4.15.2 Periodogram for HD 119285 radial velocities. 


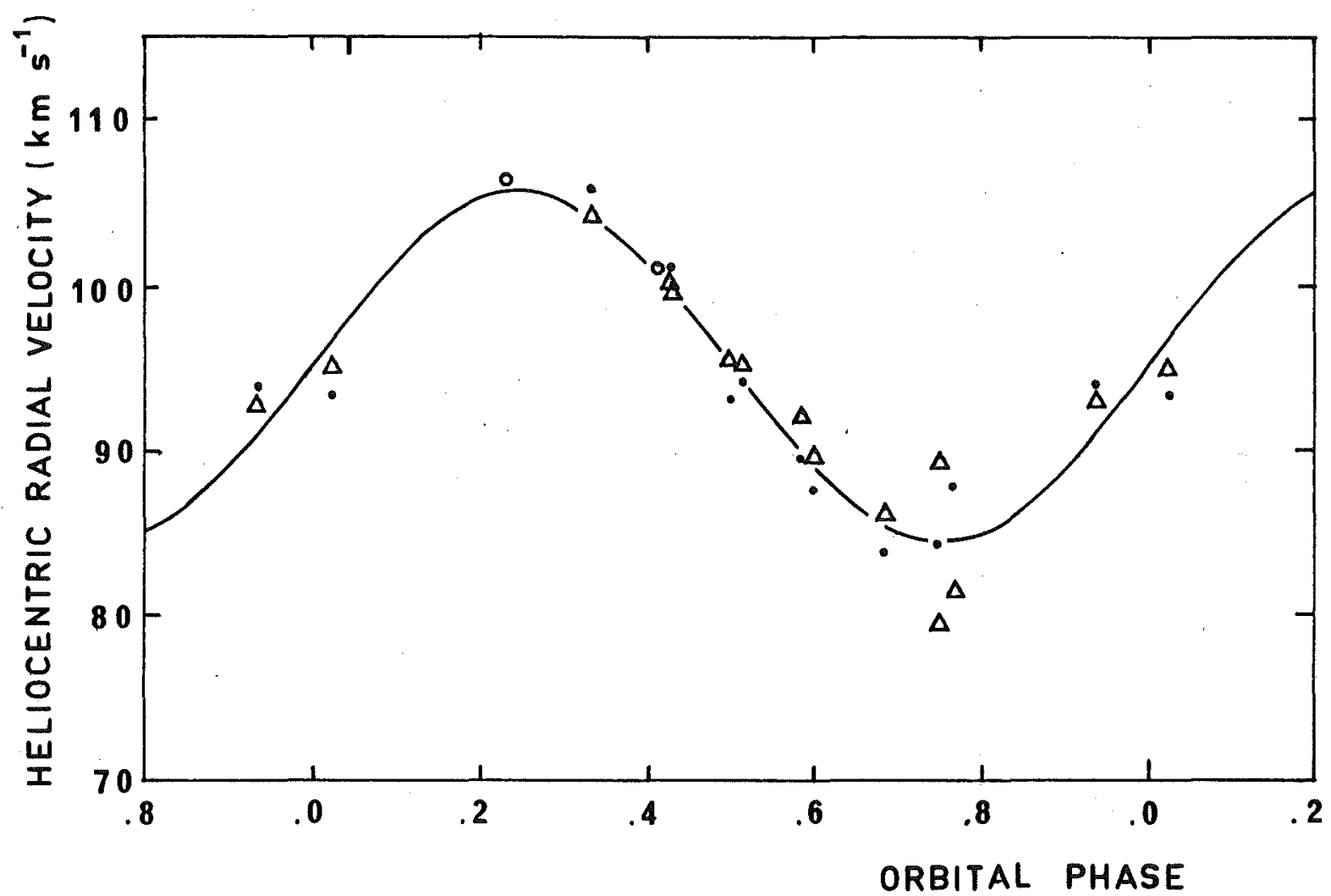

Fig. 4.15.3 Radial velocities of HD 119285. Symbols defined as in Fig. 4.11.3. 
TABLE 4.15 .1

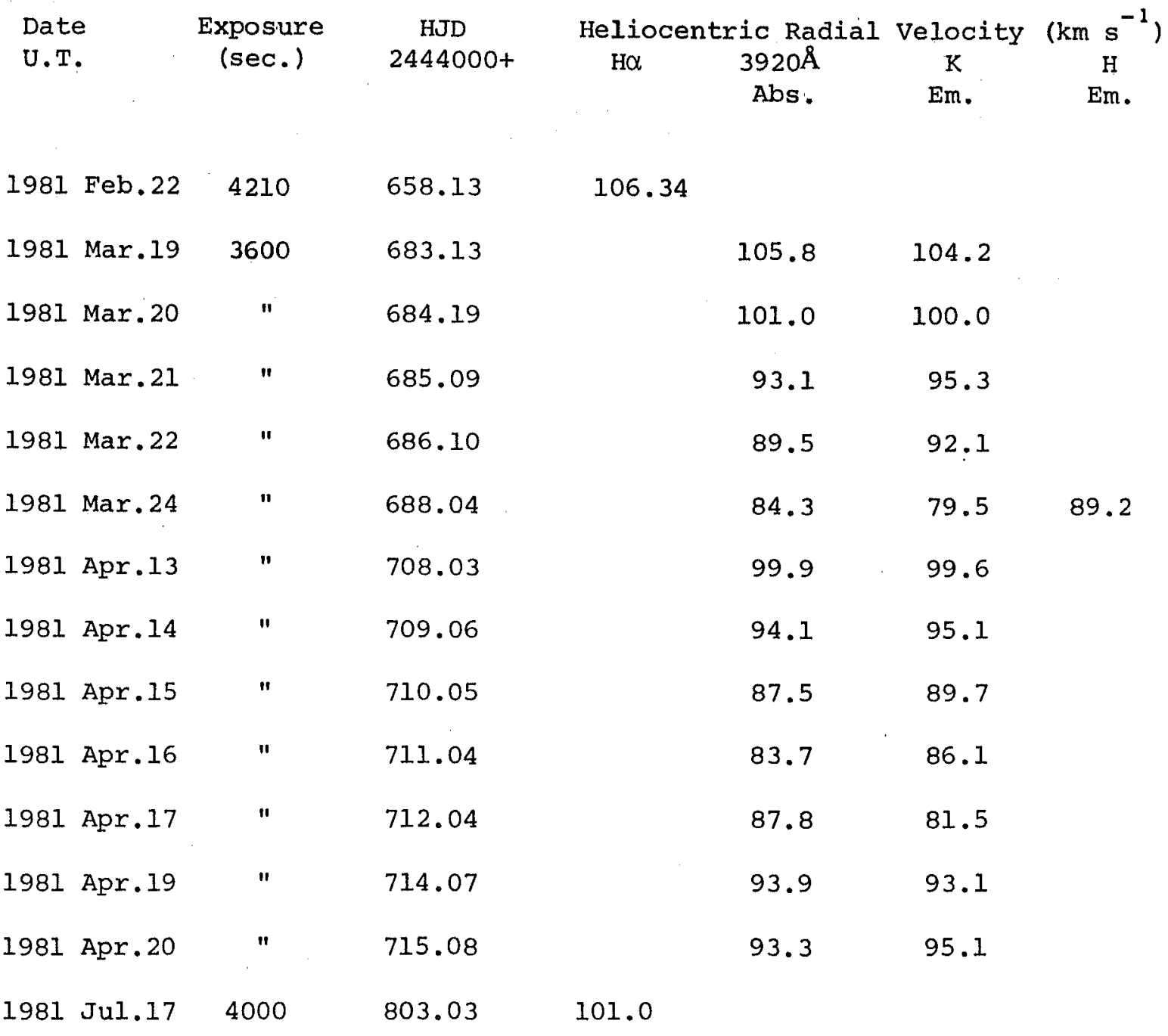

Table 4.15.1 Radial velocities of HD 119285 from $\mathrm{H} \alpha$ and Ca II spectra, MSO and SSO 1981.

$4.16 \quad \mathrm{HD} \quad 127535$

HD 127535 is listed as a Kl IV/Ve object with strong $\mathrm{Ca}$ II $\mathrm{H}$ and $\mathrm{K}$ emission by Houk and Cowley (1975), who also note the presence of possible hydrogen emission in the spectrum.

\subsubsection{H $\mathrm{H}$ Spectra}

Two spectra of the $\mathrm{H} \alpha$ region in $\mathrm{HD} 127535$ were obtained at MSO on 1981 February 23 and July 18. Both showed emission 

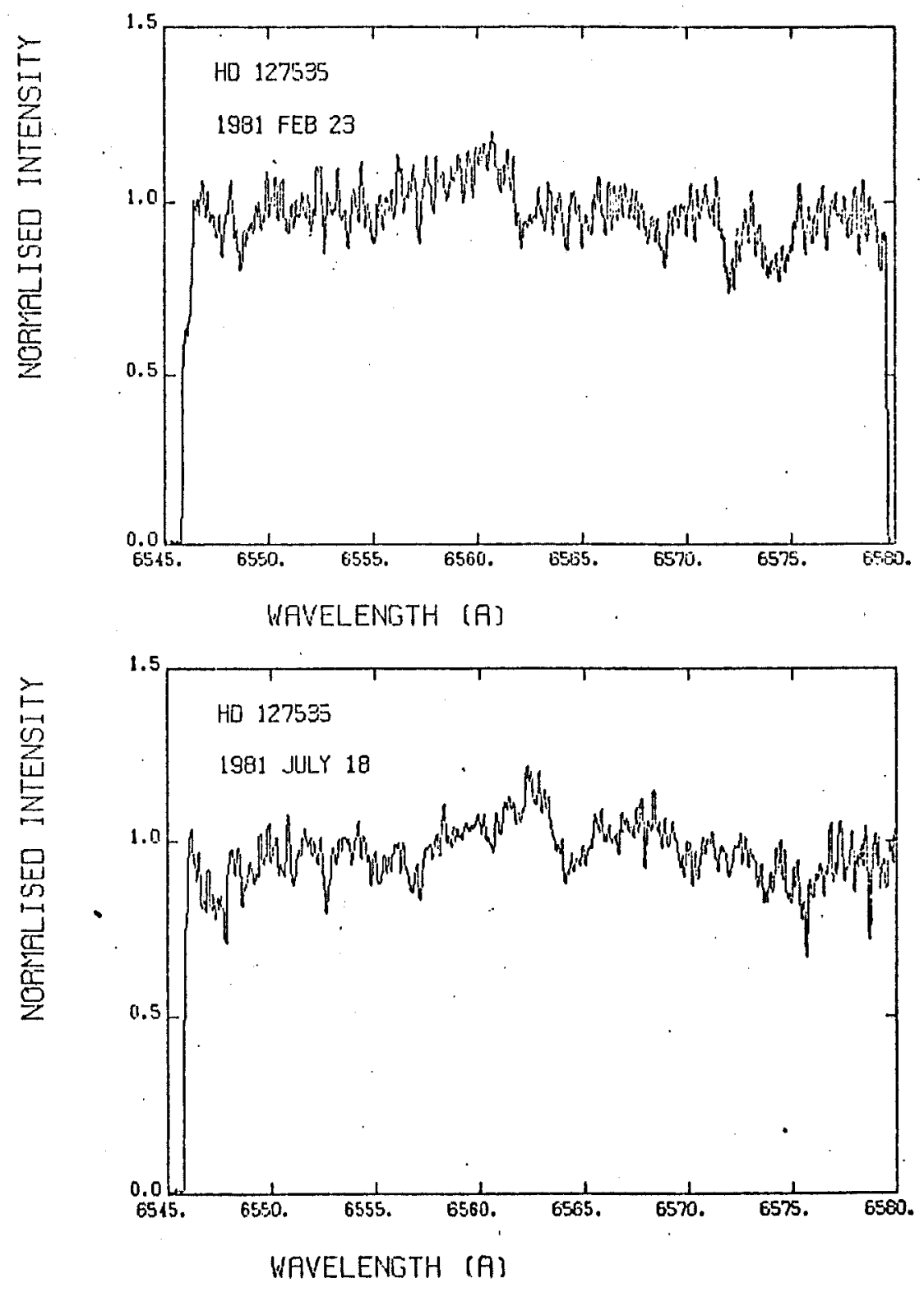

Fig. 4.16.1 H $\mathrm{H}$ spectra of HD 127535. 
above continuum level at $\mathrm{H}_{\alpha}$, with a weak, narrow absorption core (Fig. 4.16.1). The blue wing of the emission profile appears to be stronger in both spectra, suggesting a possible net mass outflow. The rotational smearing of the metallic' Iine spectrum corresponds to $\mathrm{v}_{\mathrm{e}} \sin i=15 \pm 5 \mathrm{~km} \mathrm{~s}^{-1}$.

4.16.2 $\mathrm{Ca}$ II $\mathrm{H}$ and $\mathrm{K}$ Spectra

The radial velocities of the absorption and emission spectra at the $H$ and $K$ lines are listed, along with the $H \alpha$ radial velocities, in Table 4.16 .1$.

The radial velocities of the absorption and emission spectra show good agreement with each other, and indicate that HD 127535 is a single-lined spectroscopic binary. A period analysis of the radial velocities using the method of Gieseking resulted in the periodogramillustrated in Fig. 4.16.2. An orbital period of 6.006 days was obtained, and the least-squares radial velocity solution for a circular orbit is presented in Table 3.6 and Fig. $4.16 .3(a)$.

The Wilson-Bappu width of the Ca II $\mathrm{K}$ emission core was found to be $w_{0}=0.69 \pm 0.05 \AA$, after correcting for the effects of rotational smearing. The emission flux in the $\mathrm{Ca}$ II $\mathrm{K}$ line core showed variations with total amplitude corresponding to about $20 \%$ of the mean flux.

When plotted against orbital phase (Fig. 4.16.3(c)) the flux variations appear to be modulated with the orbital period. The phase range 0.8 to 0.2 was observed on two successive orbits during 1982 April, and the flux measures on both orbits show similar phase dependence. The phase of maximum Ca II flux coincides with that of photometric wave minimum.

The $\mathrm{Ca}$ II $\mathrm{H}$ and $\mathrm{K}$ absolute surface fluxes given in Table 3.4 are systematically uncertain by about $20 \%$. The $\left(\mathrm{V}-\mathrm{R}_{\mathrm{J}}\right)$ colour index was estimated from the $(B-V)$ index measured during 1982 April at MSO. 
TABLE 4.16 .1

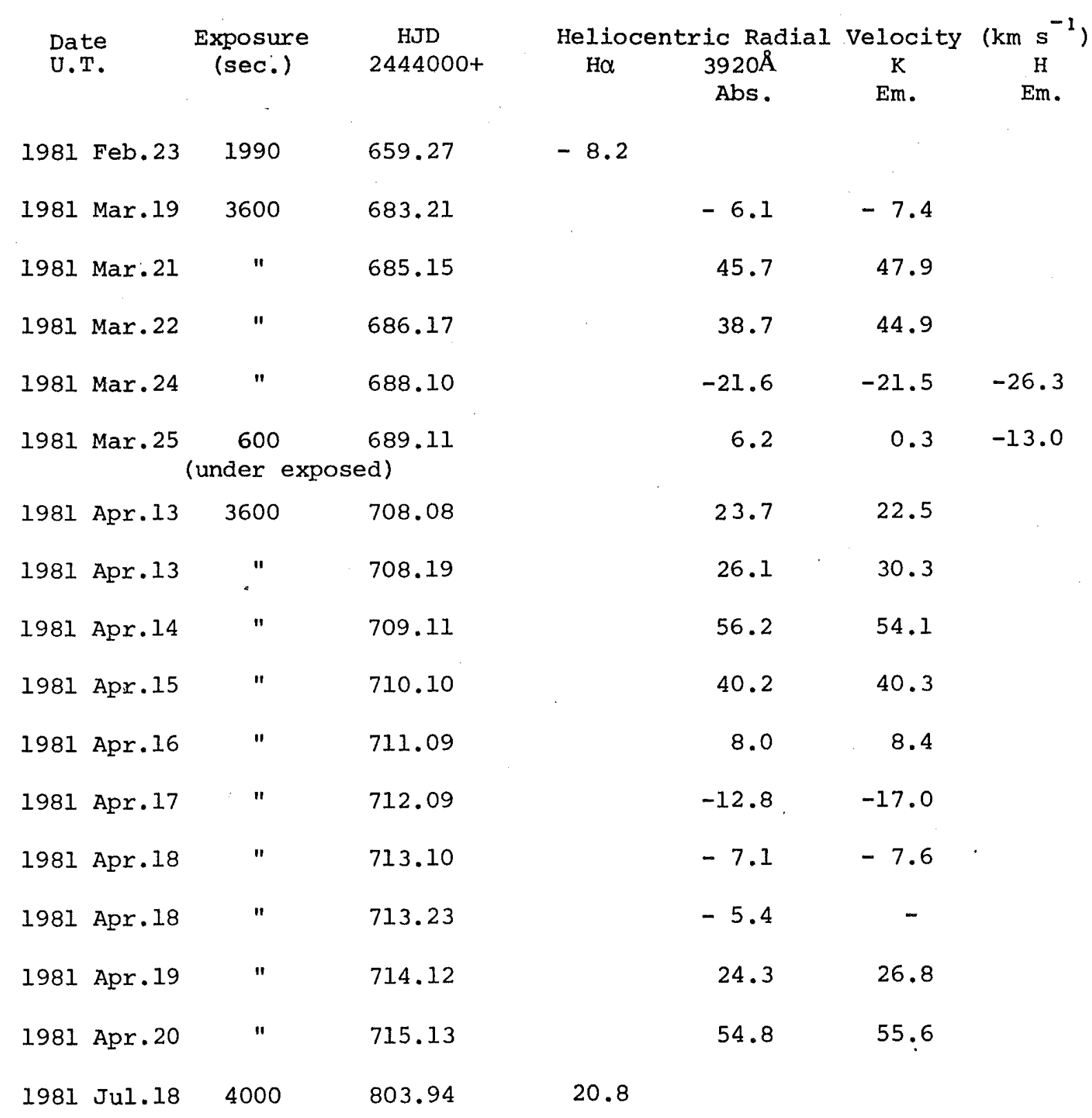

Table 4.16.1 Radial velocities of $\mathrm{HD} 127535$ from $\mathrm{H} \alpha$ and $\mathrm{Ca}$ II spectra, MSO and SSO 1981. 
$\underset{\sim}{0}$

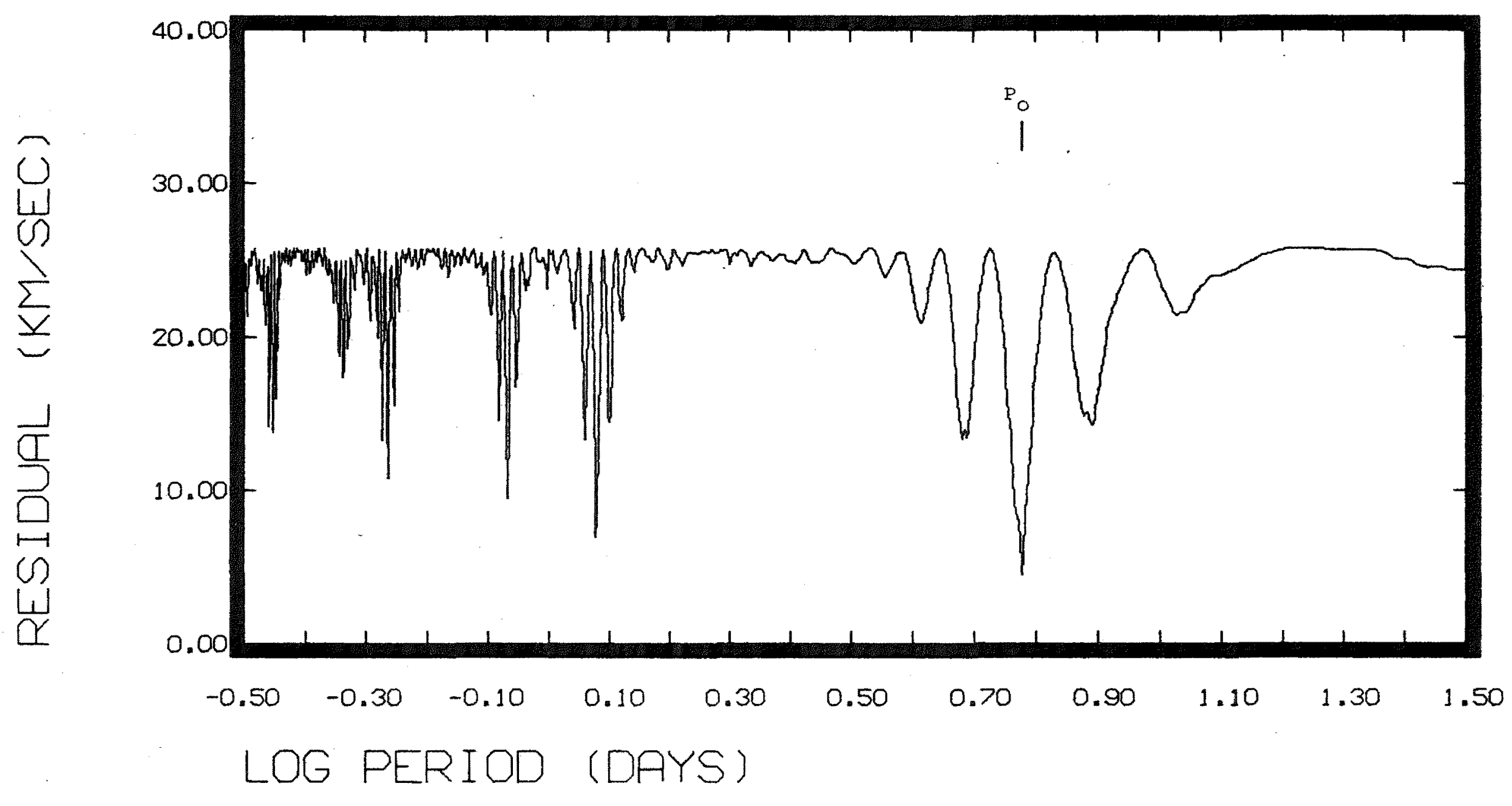

Fig. 4.16.2 Periodogram for HD 127535 radial velocities. 


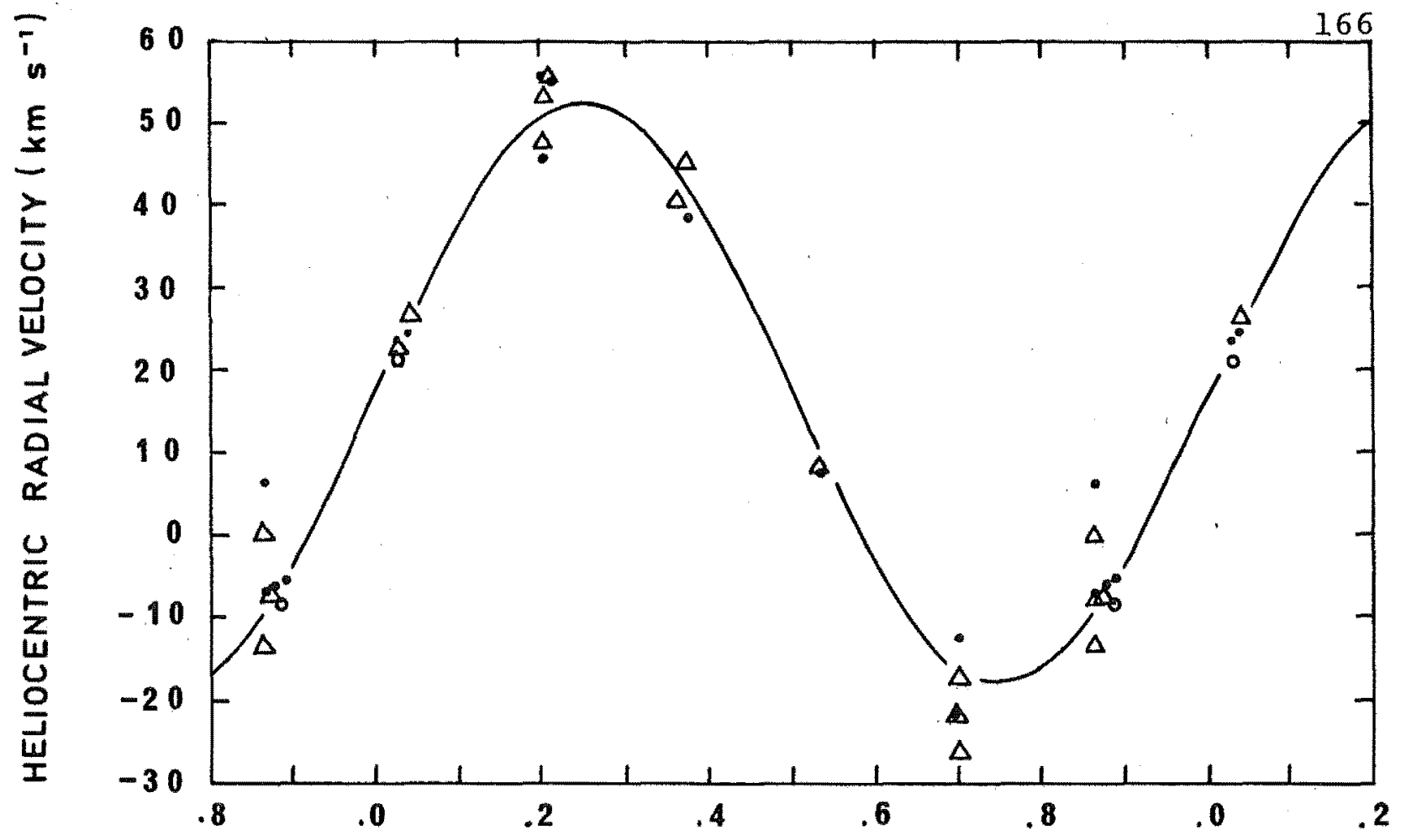

ORBITAL PHASE
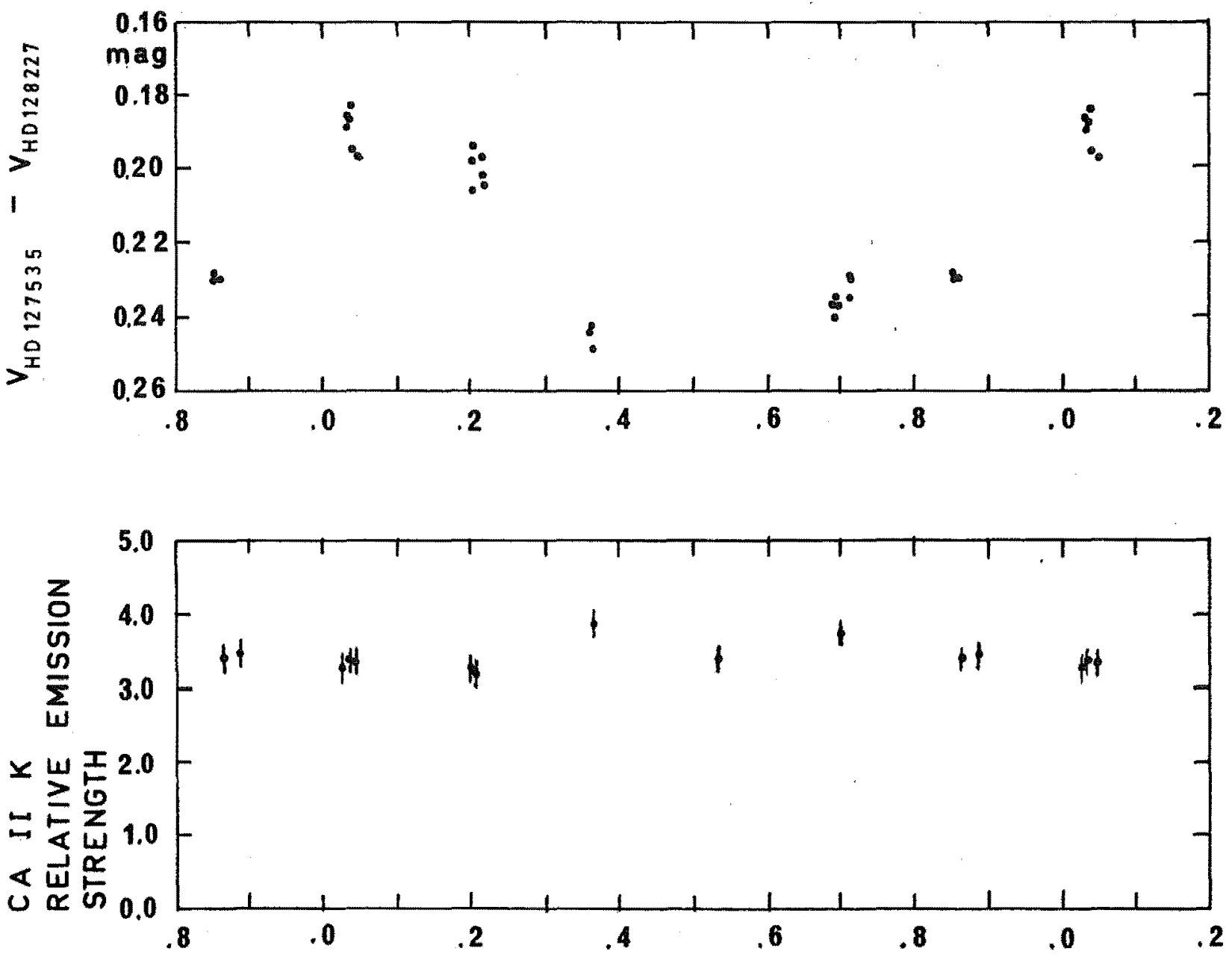

Fig. 4.16.3(a) Radial velocities of HD 127535. Symbols defined as in Fig. 4.11.3.

(b) V lightcurve of HD 127535, 1981 March-May.

(c) Ca II $\mathrm{K}$ emission flux variations in $\mathrm{HD} 127535,1981$ April 13-20. $2 \sigma$ random error bars included. 
4.16.3 Photometric Observations

UBV photometry of HD 127535 was obtained on 8 nights during 1981 April and May. The system magnitudes and colours are listed in Table 2.1 and are consistent with the KI IV spectral classification of Houk and Cowley.

The V lightcurve is illustrated in Fig. 4.16.3(b). The period of the photometric wave is equal to the orbital period, and wave minimum occurs near orbital phase 0.5 .

The shape of the wave suggests that the spot region is not compact, but rather unevenly distributed in longitude.

\subsubsection{Discussion}

The (B-V) colour index of HD 127535 yields an effective temperature near $4600 \mathrm{~K}$ using Johnson's (1966) calibration. Combined with the Wilson-Bappu width of the $\mathrm{K}$ emission core, this gives $\log g=3.2 \pm 0.5$.

Hejlesen's (1980b) evolutionary tracks in the (log 9 , $\left.\log T_{e}\right)$ plane indicate that the active component in HD 127535 is a star of between 0.9 and 2.0 solar masses which has evolved to a point near the base of the giant branch. As in the case of HD 81410, the lack of an observable secondary specrrum provides evidence that the mass ratio is less than 0.8 , if the secondary is assumed to be a main sequence object. The value of the mass function obtained from the radial velocity curve is $f(m)=(2.9 \pm 0.3) \times 10^{-2} M_{\odot}$.

Eq. 4.12-1 then yields a minimum orbital inclination $i=23^{\circ}$ for the primary mass range considered. Combined with the measured rotational smearing $v_{e} \sin i=15 \pm 5 \mathrm{~km} \mathrm{~s}^{-1}$, this range of orbital inclinations gives a radius between 1.2 and $6.0 \mathrm{R}_{\odot}$ for the active star.

The physical dimensions of the active star in this system are evidently very similar to those seen in the 
double-lined and eclipsing RS CVn systems.

In HD 127535, as in HD 81410 and HD 101309, a significant increase is seen in the Ca II emission flux near the phase of photometric wave minimum. These results provide supporting evidence for the hypothesis (based on the solar analogy) that regions of intense surface magnetic activity on the surfaces of RS CVn stars should be accompanied by enhancements of the Ca II emission flux due to localised increases in chromospheric heating. The observed increases in the $\mathrm{Ca}$ II $\mathrm{K}$ emission flux may originate in plage-like structures associated with the surface magnetic disturbances which give rise to the starspots. 
HD 137164 appears in the list of strong southern Ca II emitters by Bidelman and MacConnell (1973). The spectral type is listed as KI IV.

\section{$4.17 .1 \quad \mathrm{H} \alpha$ Spectra}

Four H $\alpha$ spectra of HD 137164 were obtained at MSO during 1981 February and July. The cross-correlation functions of these spectra (see Section 3.4.3) all showed twin, narrow peaks of equal intensity, but with variable velocity separation. Examination of the spectra (Fig.4.17.1) revealed no measurable rotational smearing in either set of metallic absorption lines, indicating that $v_{e} \sin i \leqslant 8 \mathrm{~km} \mathrm{~s}^{-1}$.

$\mathrm{H} \alpha$ itself appeared as a shallow absorption feature with a radial velocity intermediate between those of the two metalic line spectra. It appears that both stars exhibit $H_{\alpha}$ profiles whose cores are partly filled by emission (Fig. $4.17 .1)$

\subsubsection{Ca II $\mathrm{H}$ and $\mathrm{K}$ Spectra}

Three spectra of the Ca II K line region in HD 137164 were obtained at Sso during 1981 March. On all three nights, two emission cores of equal intensity were observed in the $\mathrm{K}$ line, their velocity separation decreasing on three successive nights. A subsequent cross-correlation analysis of the absorption spectrum near $3920 \AA$ revealed twin c.c.f. peaks, whose velocity separations matched those of the $\mathrm{K}$ emission cores on the three nights. The c.c.f. peaks of the absorption spectrum were of equal strength.

However, spectra obtained at sso on eight consecutive nights during 1981 April revealed apparently single absorption and emission features, with no measurable velocity variations. 
The long duration of this single-lined phase near conjunction suggests an orbital period of more than thirty days.

It thus appears that HD 137164 is a double-lined spectroscopic binary whose components have nearly equal luminosities at the wavelengths of both the Ca II K Iine and $\mathrm{H} \alpha$. The similarity of the two stars' photospheric energy distributions indicates that the two stars in HD 137164 are intrinsically very similar objects. This is borne out by the observation that both stars also display Ca II $K$ emission cores of equal strength.

The radial velocity data were not sufficient for an orbital period to be determined. This was partly due to the difficulty experienced in distinguishing one component's spectrum from the other. The data do, however, give a mass ratio close to unity, and a radial velocity $\gamma=-10.1 \pm 1.3$ $\mathrm{km} \mathrm{s}{ }^{-1}$ for the system's centre of mass. The radial velocities are listed in Table 4.17.1, and plotted in Fig. 4.17.2(a).

\subsubsection{Photometric Observations}

UBV photometry obtained on ten nights during 1981 April and May revealed a variation in the $\mathrm{V}$ band with an amplitude of at least 0.22 magnitude.

Inspection of Fig. 4.17.2 suggests that a 47-day periodicity may be present in both the photometry and the radial velocity data. However, it is doubtful that such a long period would be consistent with synchronous axial rotation in both binary components, and a reliable determination of the rotation period(s) of the stars must await a more extensive photometric investigation.

The UBV colour indices of the system are $(U-B)=0.71$ and $(B-V)=1.04$, in agreement with a $K 0 / 1$ IV spectral type and giving effective temperatures near $4700 \mathrm{~K}$ (Johnson, 1966) for both components. 
$\stackrel{-1}{-1}$
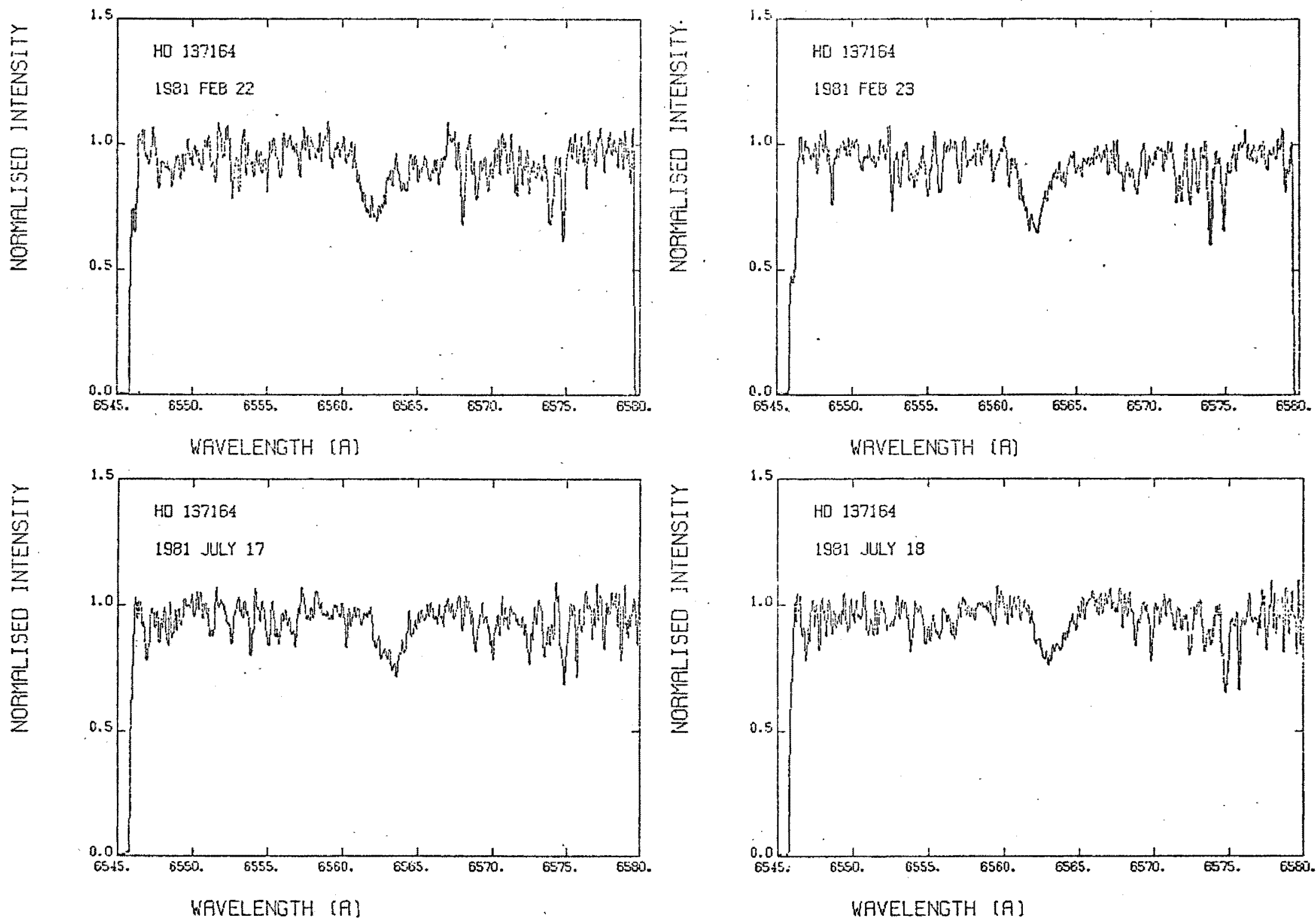
TABLE 4.17 .1

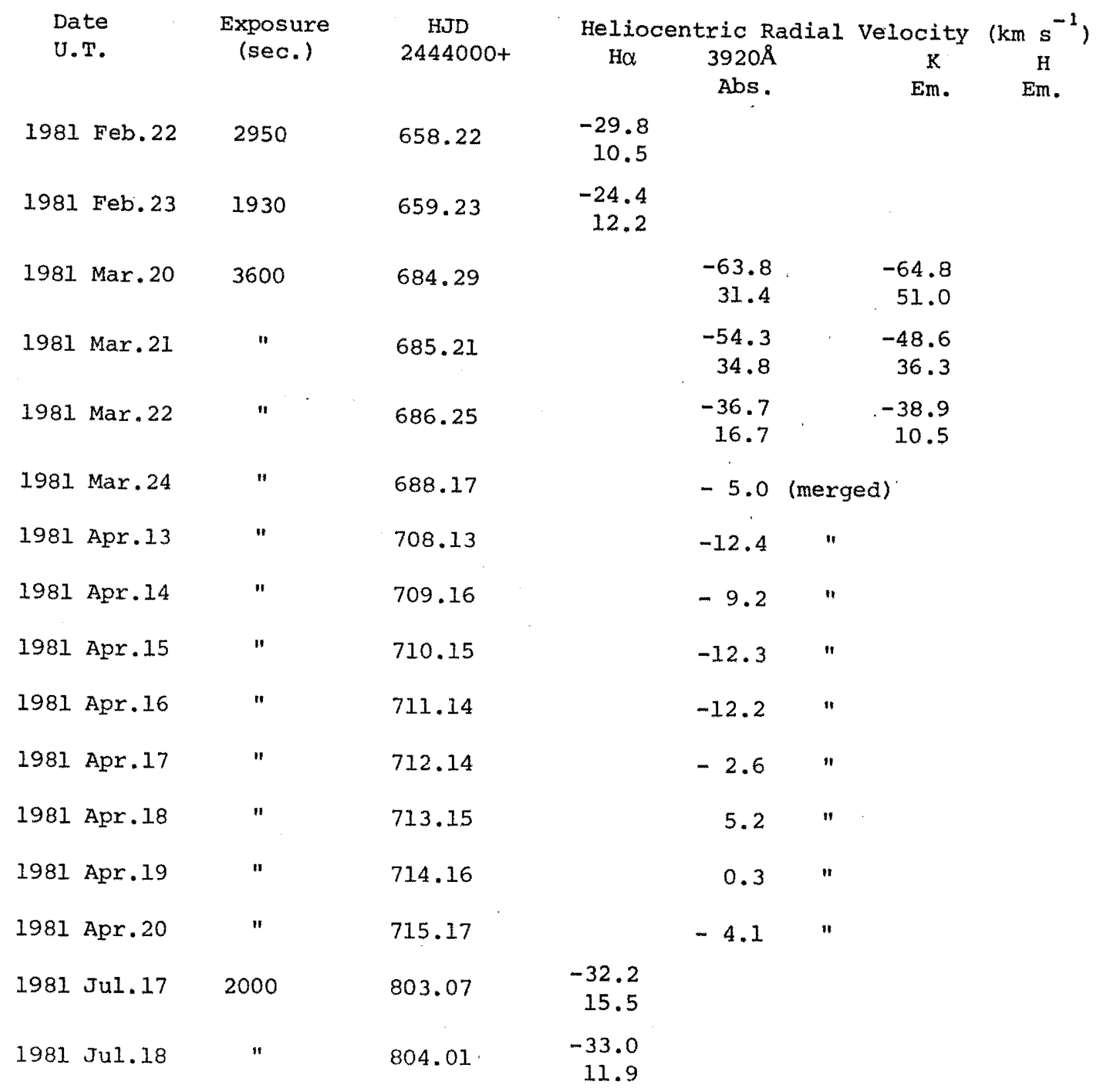

Table 4.17 .1

Radial velocities of HD 137164 from $\mathrm{H} \alpha$ and $\mathrm{Ca}$ II spectra,MSO and SSO 1981.

NOTE: Blue-shifted component is listed first at double-lined phases. Identification of individual components is not possible, owing to the similar appearances of the two spectra. 

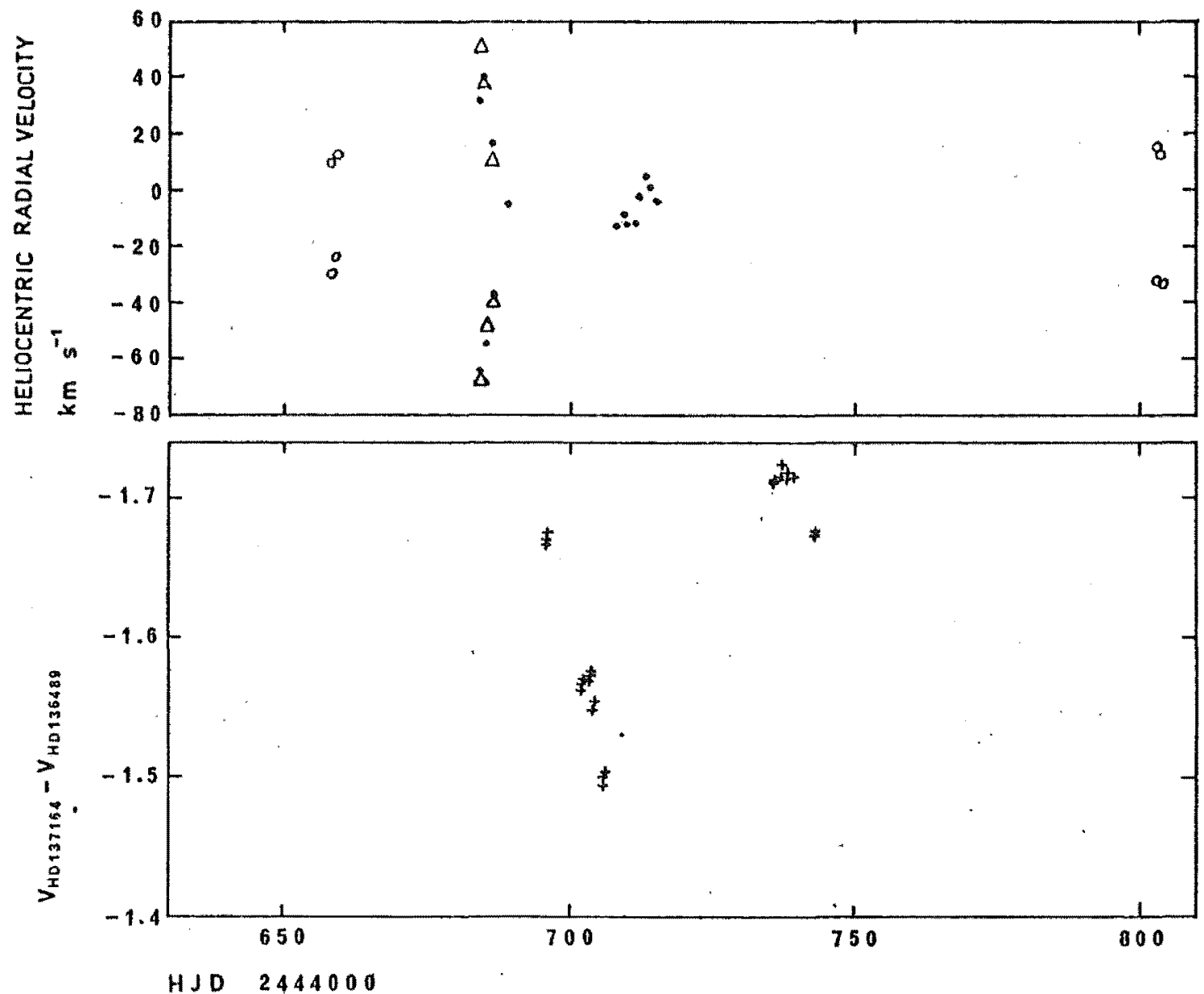

Fig. 4.17.2(a) Radial veloeities of HD 137164, 1981 February-July. Symbols defined as in Fig. 4.11.3.

(b) V lightcurve of HD 137164, 1981 March-May. 
The absolute $\mathrm{Ca}$ II $\mathrm{H}$ and $\mathrm{K}$ surface fluxes given in columns 8,9 and 10 of Table 3.4 were calculated by treating the system as a single star. Fortuitously, the system was in its single-lined phase when the spectrum used for the flux calibration was obtained. As the emission and absorption spectra of the two stars are of more or less equal intensity at all optical wavelengths, the assumption that their surface fluxes are also equal is adequate for the purposes of this investigation.

Further photometric and spectroscopic observations should be made of this system, in order to determine its orbital period and the rotational periods of the individual stars. 
HD 155555 was first identified as a spectroscopic binary by Bennett, Evans and Laing (1962), who determined an orbital period of 1.6817 days. Bennett et.al. noted the presence of $\mathrm{Ca}$ II $\mathrm{H}$ and $\mathrm{K}$ emission in the spectra of both components. Eggen (1978) determined colour indices $(B-V)=0.84$ and $(R-I)=0.33$ in the Kron system;

he also analyses the space motion and finds that HD 155555 is probably a young disk population object.

Stacy, Stencel and Weiler (1980) made further radial velocity measurements of this system. They also obtained two $2 \AA \mathrm{mm}^{-1}$ échelle spectra of the $\mathrm{H}$ and $\mathrm{K}$ lines using the $4 \mathrm{~m}$ telescope at Cerro Tololo Inter-American Observatory, and high resolution IUE spectra of the Mg II $\mathrm{h}$ and $\mathrm{k}$ line region.

\subsubsection{Photometric Observations}

BVR $_{\mathrm{C}} \mathrm{I}_{\mathrm{C}}$ photometry obtained at MJUO during 1979 August and october and 1980 January showed a distortion wave with a total amplitude of 0.08 magnitude and the same period as the binary orbit (Fig. 4.18.1).

The colour indices are listed in Table 2.1, and show reasonable agreement with Eggen's results in the Kron system.

\section{$4.18 .2 \quad \mathrm{H \alpha}$ Spectra}

Two rather under-exposed spectra of the $\mathrm{H} \alpha$ region were obtained with the cassegrain échelle spectrograph on the $61 \mathrm{~cm}$ Boller and Chivens telescope at MJUO on 1979 May 8 and 1980 June 11. These spectra showed Ha filled with emission to continuum level. The weak metallic lines near $\mathrm{H} \alpha$ were so greatly smeared by rotation as to be undetectable. However, stronger features such as the $\mathrm{Na} I \mathrm{D}$ lines were identifiable, and were clearly double. 
The relative strengths of the $\mathrm{Na} I \mathrm{D}$ lines in the two stars' spectra indicated a luminosity ratio of $0.3 \pm 0.1$ at this wavelength $(\sim 5890 \AA)$.

Higher-quality échelle spectra of the $\mathrm{H} \alpha$ region were obtained at the coudé focus of the $1.88 \mathrm{~m}$ telescope at MSO on $1981 \mathrm{July} 16$ and 18. In all three of these spectra (Fig.4.18.2). a weak $\mathrm{Ha}$ absorption core is seen, whose radial velocity matches that of the star exhibiting the stronger absorption spectrum at the $\mathrm{Na} I \mathrm{D}$ lines.

\section{$4.18 .3 \mathrm{Ca}$ II $\mathrm{H}$ and $\mathrm{K}$ spectra}

Spectra of the $\mathrm{Ca}$ II $\mathrm{K}$ line region obtained at sso during 1981 March showed a double absorption spectrum, the intensities of the two spectra being about equal.

The $\mathrm{K}$ line core emission peaks were also nearly equal in apparent strength, as noted by stacy et.al. (1980). In order to evaluate the absolute surface fluxes in the $H$ and $K$ line cores, it was necessary to evaluate the values of $\left(V-R_{J}\right)$ for the two stars. This was done using a method similar to that used for HD 101309, described in Section 4.13.3. The estimated flux ratios of the two spectra at $3920 \AA$ and at $5890 \AA$ give $\Delta V=1.1 \pm 0.2 \mathrm{mag}$.

Bennett, Evans and Laing (1962) assign spectral types of $G 5$ and k0 to the two stars. They also find that the mass ratio is close to unity, suggesting that the primary's radius must be less than or equal to its Roche lobe radius.

If we assume masses of $1.1 \pm 0.1 \mathrm{M}_{\odot}$ for both components, their separation will be $a=7.7 \pm 0.3 \mathrm{R}_{\odot}$, and equation $5-4$ (Paczynski, 1971) gives Roche lobe radii of $R_{L}=2.9 \pm 0.1 R_{\odot}$ for both stars. If we assume a lower limit of $\sim 1 R_{\odot}$ on the radius of the secondary, we find that the lowest possible value for $k$ is 0.34 . 
Good agreement with the observed colour indices and flux ratios was obtained for a model with a G5 V secondary $\left(B-V=0.66, V-R_{J}=0.54, V-I_{J}=0.89\right)$ and a $\mathrm{K} 0 \mathrm{IV}$ primary $\left(\mathrm{B}-\mathrm{V}=0.83, \mathrm{~V}-\mathrm{R}_{\mathrm{J}}=0.70, \mathrm{~V}-\mathrm{I}_{\mathrm{J}}=1.19\right)$, and $\Delta \mathrm{V}=1.1$ magnitude. For this model, the Barnes-Evans relation gives $\mathrm{k}=0.36$, suggesting that the cooler star is nearly filling its Roche lobe.

The $\mathrm{Ca}$ II $\mathrm{H}$ and $\mathrm{K}$ absolute surface fluxes given in Table 3.4 were calculated under the assumption that the ratio $\frac{\text { Total flux in } H \text { and } K \text { emission cores }}{\text { Flux in band } 3925-3975 \AA}$

was approximately the same for both stars. The individual $V-R_{J}$ colour indices calculated above were used for the absolute flux calibration for each star. The values in columns 9 and 10 of Table 3.4 are uncertain by about $50 \%$ as a result.

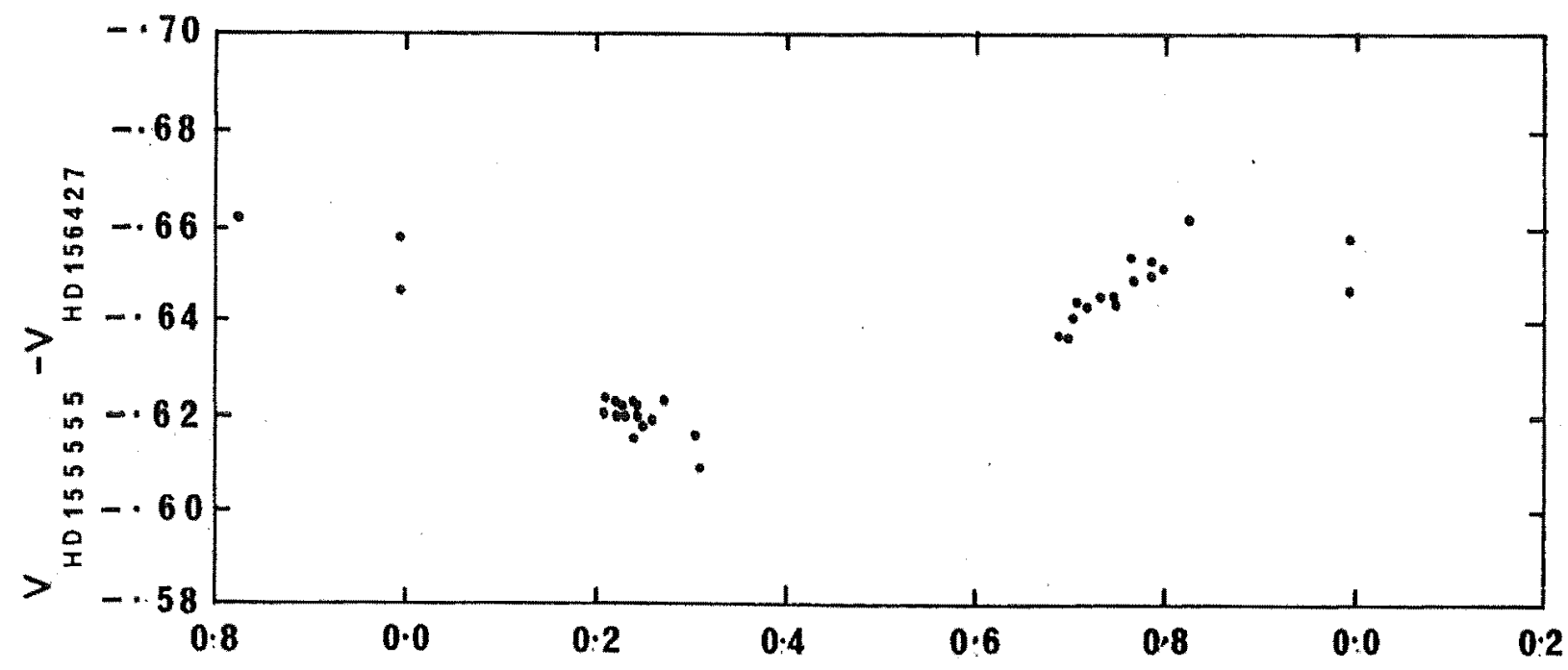

ORBITAL PHASE 

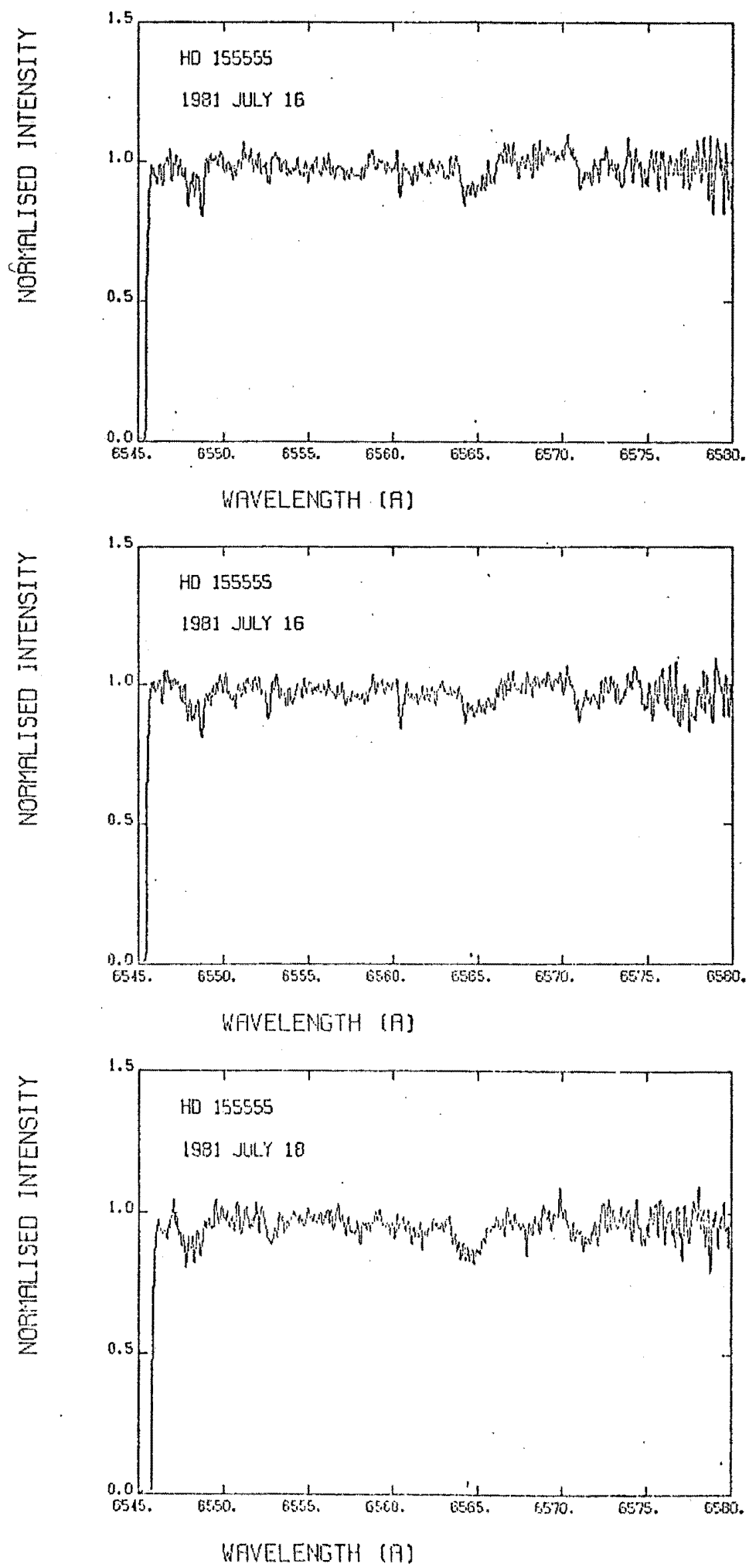

Fig. 4.18 .2 
HD 165141 is classified as a G8/K0 II/IIIp object with moderate $\mathrm{Ca}$ II $\mathrm{H}$ and $\mathrm{K}$ emission by Houk and Cowley (1975). This star also appears in Bidelman and MacConnell's (1973) list of southern $\mathrm{Ca}$ II $\mathrm{H}$ and $\mathrm{K}$ emitters.

\section{$\underline{4.19 .1 \quad \mathrm{H} \alpha \text { Spectra }}$}

A single échelle spectrum of the $\mathrm{H \alpha}$ region in $\mathrm{HD}$ 165141 was obtained at MJUO on $1980 \mathrm{July} 29$. Although the spectrum was under-exposed, the spectrum did not appear to be smeared by rotation, and $\mathrm{H} \alpha$ appeared as a normal absorption feature.

\subsubsection{Ca II $\mathrm{H}$ and $\mathrm{K}$ Spectra}

A single spectrum of the $\mathrm{H}$ and $\mathrm{K}$ line region in HD 165141 was obtained at MSO during 1981 March, and seven échelle spectra of the Ca II K line profile were obtained at sso during 1981 April.

No evidence for radial velocity variations was found; the radial velocity data are listed in Table 4.19.1. The Wilson-Bappu width of the $C a$ II $K$ line core yielded a surface gravity $\log g=2.9 \pm 0.2$, for an assumed effective temperature of $4600 \pm 200 \mathrm{~K}$ appropriate to Houk and Cowley's spectral type.

This same effective temperature was used to estimate the $(V-R)_{J}$ colour index; the absolute $C a$ II $H$ and $K$ surface fluxes given in Table 3.4 are thus uncertain by about 50 \% These flux values are rather lower than those obtained for the other stars investigated here, but are nevertheless about an order of magnitude higher than the values obtained by Linsky et.al. (1979) for single stars of similar spectral type. Further radial velocity studies and photometric observations of this star should be made in order to determine whether or not it is a single star and whether it displays RS CVn-type photometric variability. 
TABLE $\quad 4.19 .1$

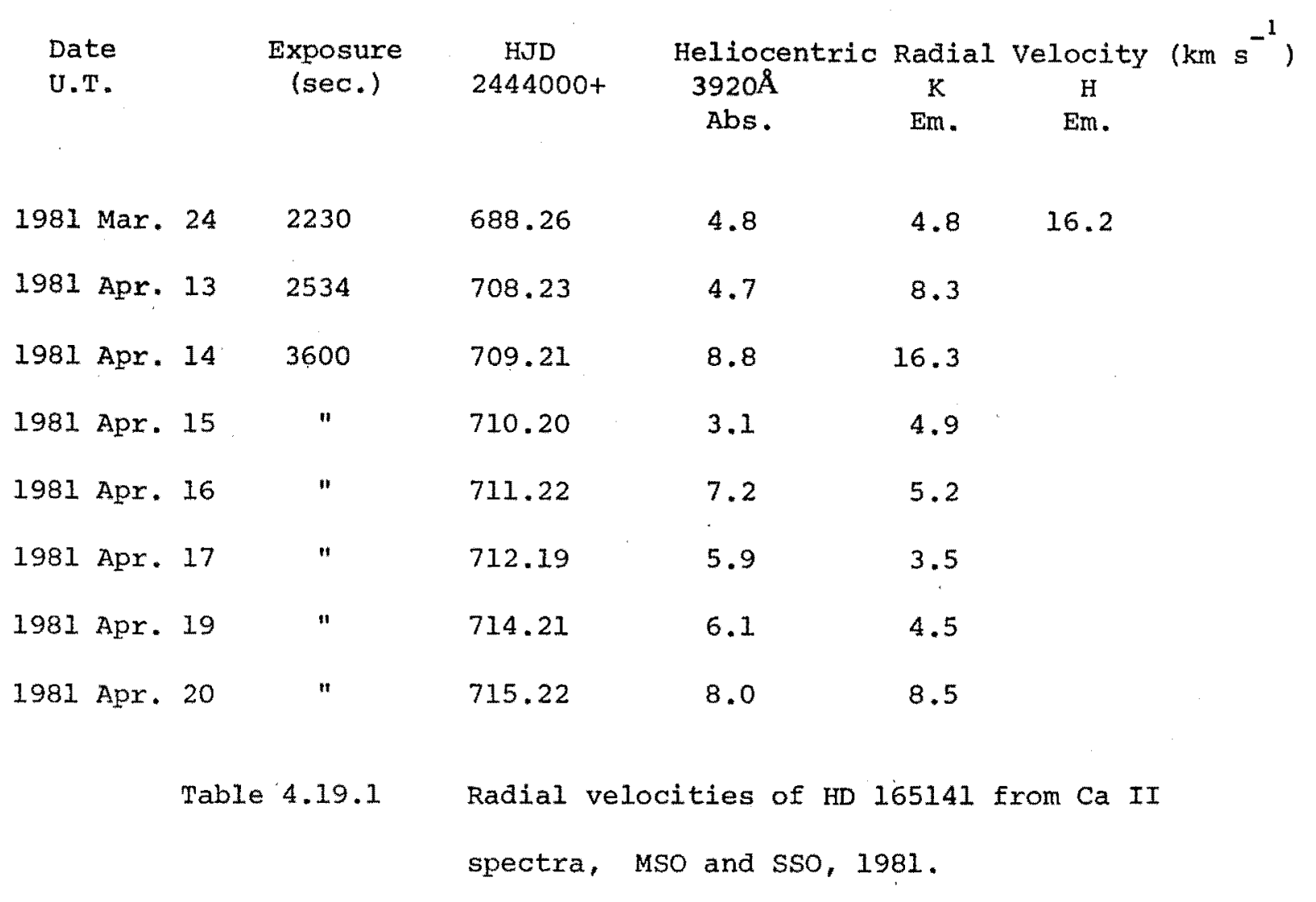

$4.20 \quad$ HD 181809

HD 181809 appears in Bidelman and MacConnell's (1973)

list of southern $\mathrm{Ca}$ II $\mathrm{H}$ and $\mathrm{K}$ emitters, where its spectral type is given as $\mathrm{KI}$ III + F.

F. Fekel has found evidence for radial velocity variations, and rotational smearing corresponding to $v_{e} \sin i=15 \mathrm{~km} \mathrm{~s}^{-1}$ (B.Bopp, private communication, 1981).

\subsubsection{Photometric Observations}

BVR $_{C} I_{C}$ photometry obtained at MJUO during 1979 August and October revealed a low-amplitude photometric variation with an amplitude of about 0.014 magnitude in $v$. Insufficient data were obtained for any determination of the photometric 
period to be made.

The colour indices of HD 181809 agree with the KI III spectral type, and are listed in Table 2.1 .

\subsubsection{Ha Spectra}

An $\mathrm{H} \alpha$ spectrum of HD 181809 was obtained at MSO on 1981 July 16. The $H \alpha$ profile is rather shallow, and may be partially filled with emission (Fig. 4.20.1); the rotational smearing of the metallic lines corresponds to $v_{e} \sin i=10 \pm 3$ $\mathrm{km} \mathrm{s} \mathrm{s}^{-1}$, a rather lower figure than that obtained by Fekel. An earlier $H \alpha$ échelle spectrum of this star, obtained at MJUO on 1980 June 11, showed similar features. The radial velocities of both spectra are listed in Table 4.20.1.

\section{$4.20 .3 \quad \mathrm{Ca}$ II $\mathrm{H}$ and $\mathrm{K}$ Spectra}

Seven spectra of the Ca II K line region in HD 181809 were obtained during 1981 April at sso. The radial velocities of the absorption spectrum and the $K$ emission peak are listed in Table 4.20.1.

The Wilson-Bappu width of the $K$ emission core yielded a surface gravity $\log g=3.0 \pm 0.2$. An effective temperature of $4600 \mathrm{~K}$ was used, based on the measured colour indices and the relations of Johnson (1966).

No significant $K$ emission flux variations were noted (Fig. 3.2), with the exception of an anomalously high relative flux reading at HJD 2444710.28 . The enhanced flux level appears to be real, and may have resulted from a short-duration flare event.

The colour indices of HD 181809 are very similar to those of HD 81410. Although no spectra of the $H$ and $K$ line region were obtained at MSO, an estimate of the absolute surface emission flux was included in Table 3.4 , based on the ratio of the sso relative flux measures (Table 3.5 ) in the $K$ 
line core for HD 181809 and HD 81410. The resulting surface fluxes (given in columns 9 and 10 of Table 3.4) are uncertain by about $30 \%$, but are comparable with those obtained for several of the binaries listed in Table 3.4. The low amplitudes of the velocity variation, the photometric wave and the Ca II $K$ emission flux variation suggest that HD 181809 is likely to be a single-lined RS CVn system, orbiting nearly pole-on to the line of sight. The low rotational broadening of its spectrum permits radial velocity measurements of high accuracy to be obtained comparatively easily. Further radial velocity studies should be made of this star in order to determine its orbital period, which appears to be between six and ten days.

TABLE 4.20 .1

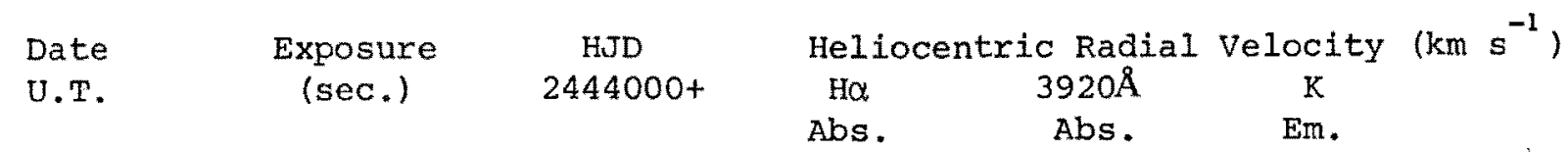

$\begin{array}{lllllll}1980 \text { Jun. } 11 & 12 & 000 & 402.07 & -21.8 & \\ 1981 \text { Apr. } 14 & 2397 & 709.26 & -18.4 & -17.1 \\ 1981 \text { Apr. } 15 & 1 & 200 & 710.28 & -20.0 & -21.3 \\ 1981 \text { Apr. } 16 & 3600 & 711.27 & -26.4 & -23.8 \\ 1981 \text { Apr. } 17 & " & 712.24 & -25.5 & -26.3 \\ 1981 \text { Apr. } 18 & " & 713.28 & -22.8 & -24.7 \\ 1981 \text { Apr. } 19 & " & 714.26 & -20.6 & -20.7 \\ 1981 \text { Apr. } 20 & " & 715.27 & & -17.3 & -16.8 \\ 1981 \text { Jul. } 16 & 2000 & 802.20 & -19.6 & & \end{array}$

Table 4.20.1 Radial velocities of $\mathrm{HD} 181809$ from $\mathrm{H} \alpha$ and Ca II spectra, MJUO, MSO and SSO 1980/81. 


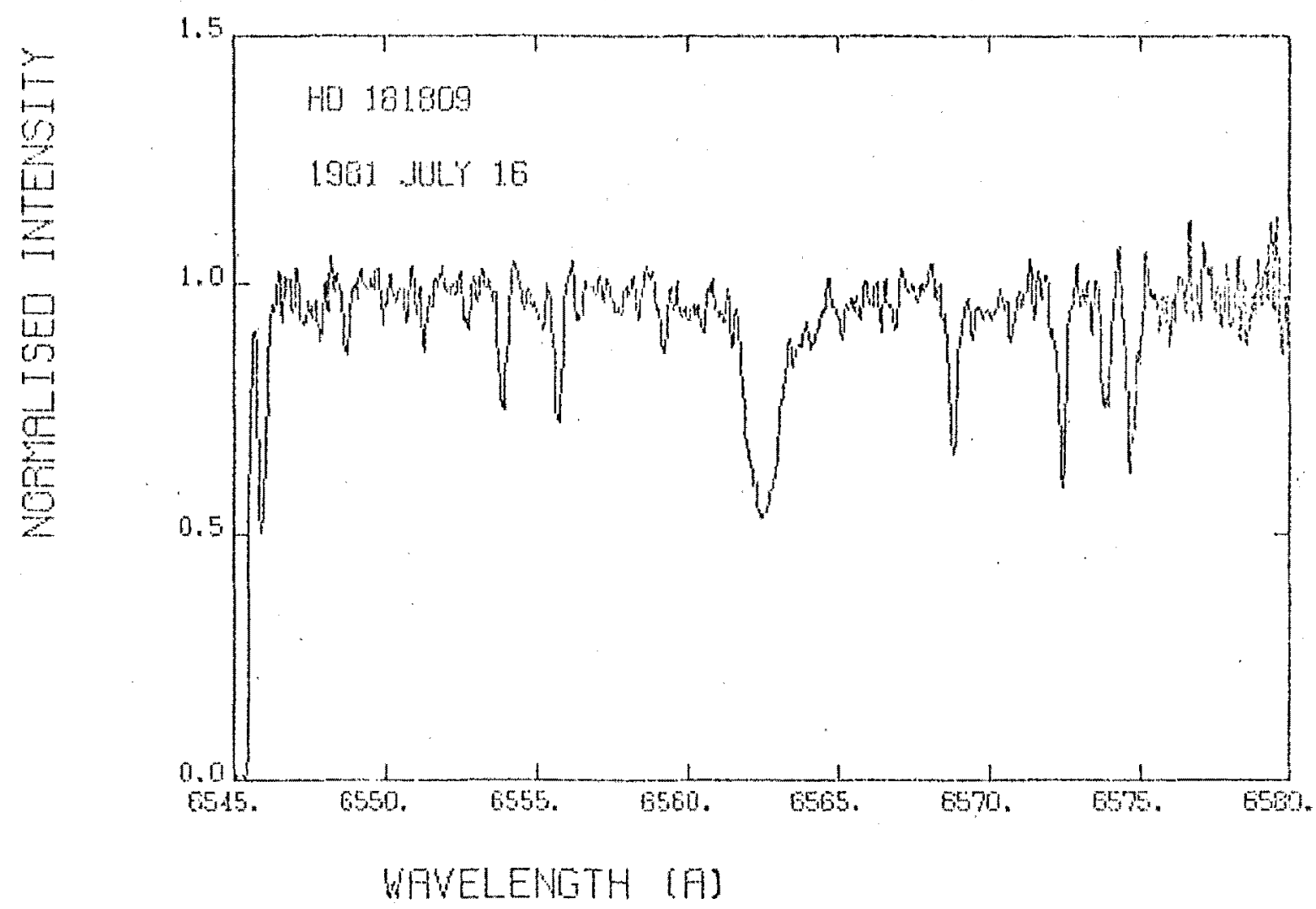

Fig. 4.20.1 H $\alpha$ spectrum of HD 181809.

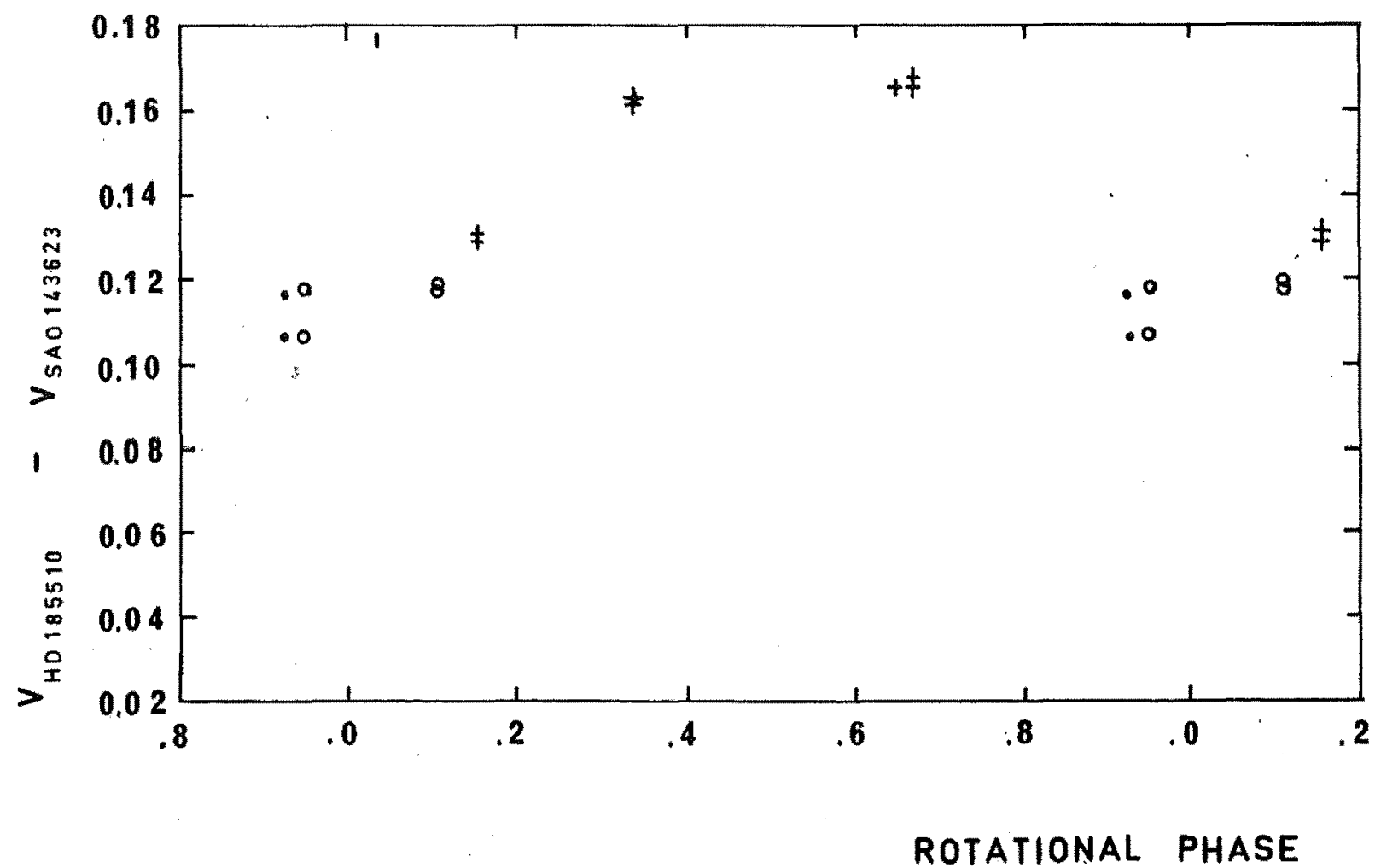

Fig. 4.21.1 V 1ightcurve of HD 185510, 1979 August-October, 
$4.21 \quad \mathrm{HD} 185510$

HD 185510 appears in Bidelman and MacConnell's (1973) list of southern Ca II emitters, with a KO III/IV spectral classification. F. Fekel has found that the star has a variable radial velocity, and rotational smearing $v_{e} \sin i=21 \mathrm{~km} \mathrm{~s}^{-1}$ (B. Bopp, private communication, 1981).

\subsubsection{Photometric Observations}

Photometry of HD 185510 in the BVR ${ }_{C}{ }_{C}$ system was obtained at MJUO during 1979 August and october.

A sinusoidal fit to the data was obtained using the method of Gieseking (Section 2.4.4), the most likely period for the variation being $6.12 \pm 0.02$ days. The periodogram is plotted in Fig. 2.2 (c) and the variation in $V$ is plotted against rotational phase in Fig. 4.21.1, using the ephemeris

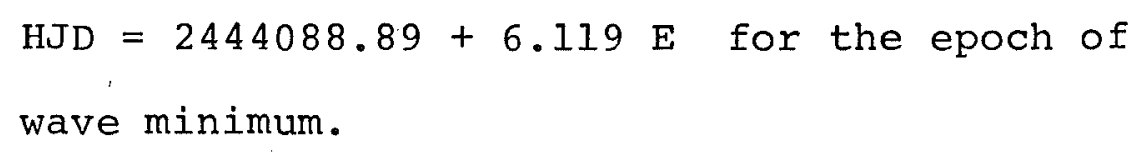
wave minimum.

The colour indices listed for HD 185510 in Table 2.1 agree with the K0 III/IV spectral type.

$\underline{4.22 \quad H D \quad 195040}$

HD 195040 is given a K2 III + F spectral classification in Bidelman and MacConnell's (1973) list of southern Ca II emitters.

\subsubsection{Radial Velocity Observations}

Three spectra of the Mg I b line region in HD 195040 were obtained during 1980 November at MSO. The crosscorrelation functions of these spectra with the template spectrum gave the radial velocities listed in Table 4.22.I, and showed slight rotational smearing. 
$4.22 .2 \quad \mathrm{H} \alpha$ Spectra

Six spectra of the Ho region in HD 195040 were obtained during $1981 \mathrm{July}$ at MSO. The variability of the $\mathrm{H} \alpha$ emission profile in these spectra is discussed by collier et.al. (1982); at the time when these spectra were obtained, HD 195040 was detected as a $\sim 15 \mathrm{mJy}$ radio source with the $64 \mathrm{~m}$ Parkes radio telescope, and may have been exhibiting an unusually high activity level.

The radial velocities obtained from the $\mathrm{H} \alpha$ spectra are listed in Table 4.22.1 and show HD 195040 to be a singleIined spectroscopic binary. The rotational smearing of the spectra was difficult to ascertain owing to the rather poor signal-to-noise ratio, but appears to have a value near $v_{e} \sin i=15 \pm 5 \mathrm{~km} \mathrm{~s}^{-1}$.

\subsubsection{Photometric Observations}

BVR $_{C} I_{C}$ observations of HD 195040 obtained during 1979 October and November yielded the colour indices listed in Table 2.1, which are consistent with a K0 III/IV spectral type. Variability with an amplitude of 0.14 magnitude in $V$ was observed; a period analysis using the method of Gieseking gave the periodogram plotted in Fig. 4.22.1(b). Analysis of the aliasing structure (Section 2.4.4) suggests that the most probable photometric period is $8.27 \pm 0.02$ days. Such a period appears to be consistent with the radial velocity data, although the latter were insufficient for a successful independent period analysis to be performed (Fig. 4.22.1(a)). A least-squares fit to the radial velocity data, with an assumed period close to 8.27 days, gave the best-fit solution summarised in Table 4.22 .2 . The radial velocity data and $\mathrm{V}$ photometric data are plotted against orbital phase using this ephemeris in Fig. 4.22.2. It should be emphasised that the 
:

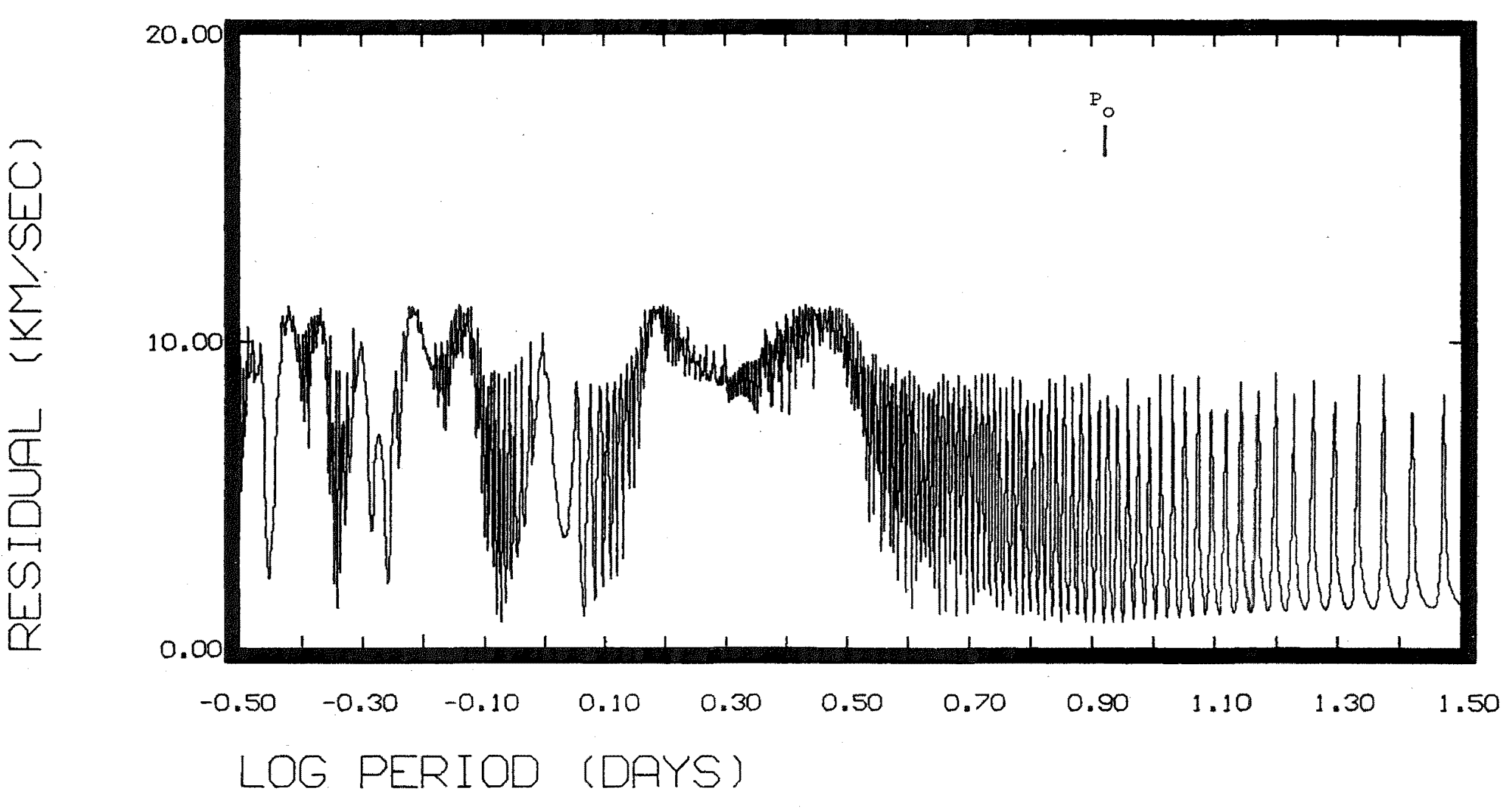

Fig. 4.22.1(a) Periodogram for HD 195040 radial velocities. 


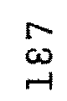

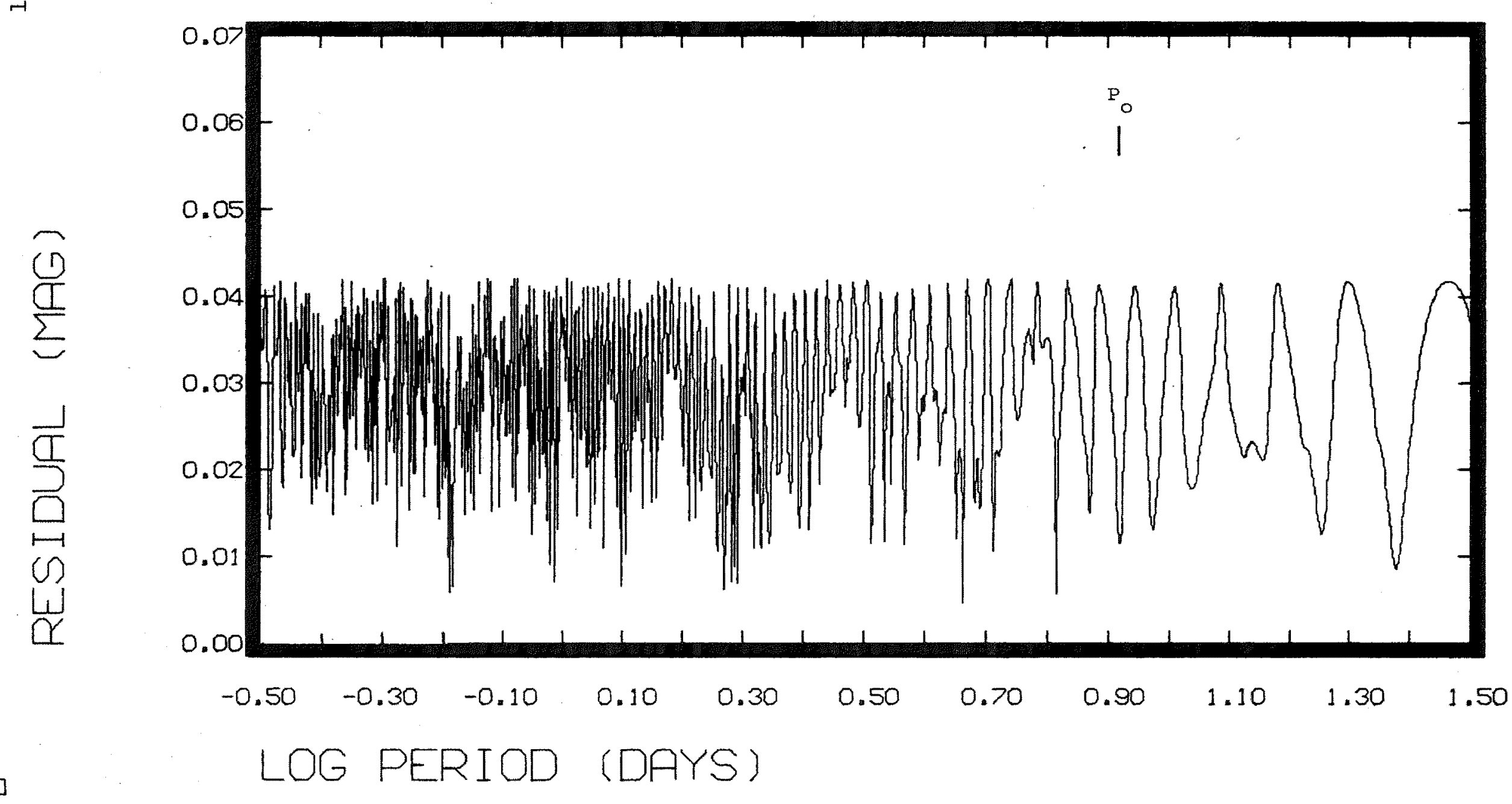

Fig.4.22.1 (b) Periodogram for HD $195040 \mathrm{~V}$ photometry. 

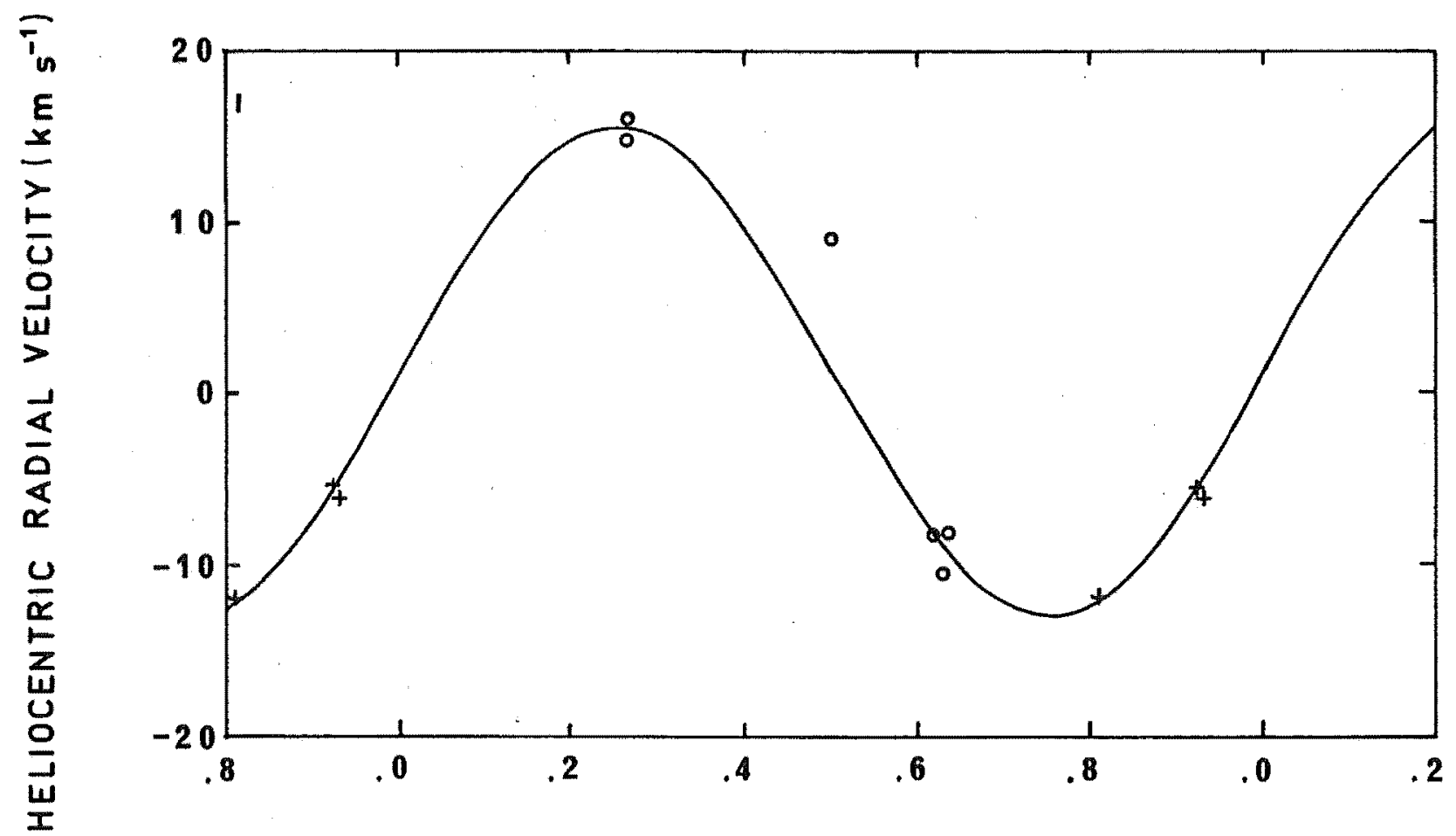

ORBITAL PHASE

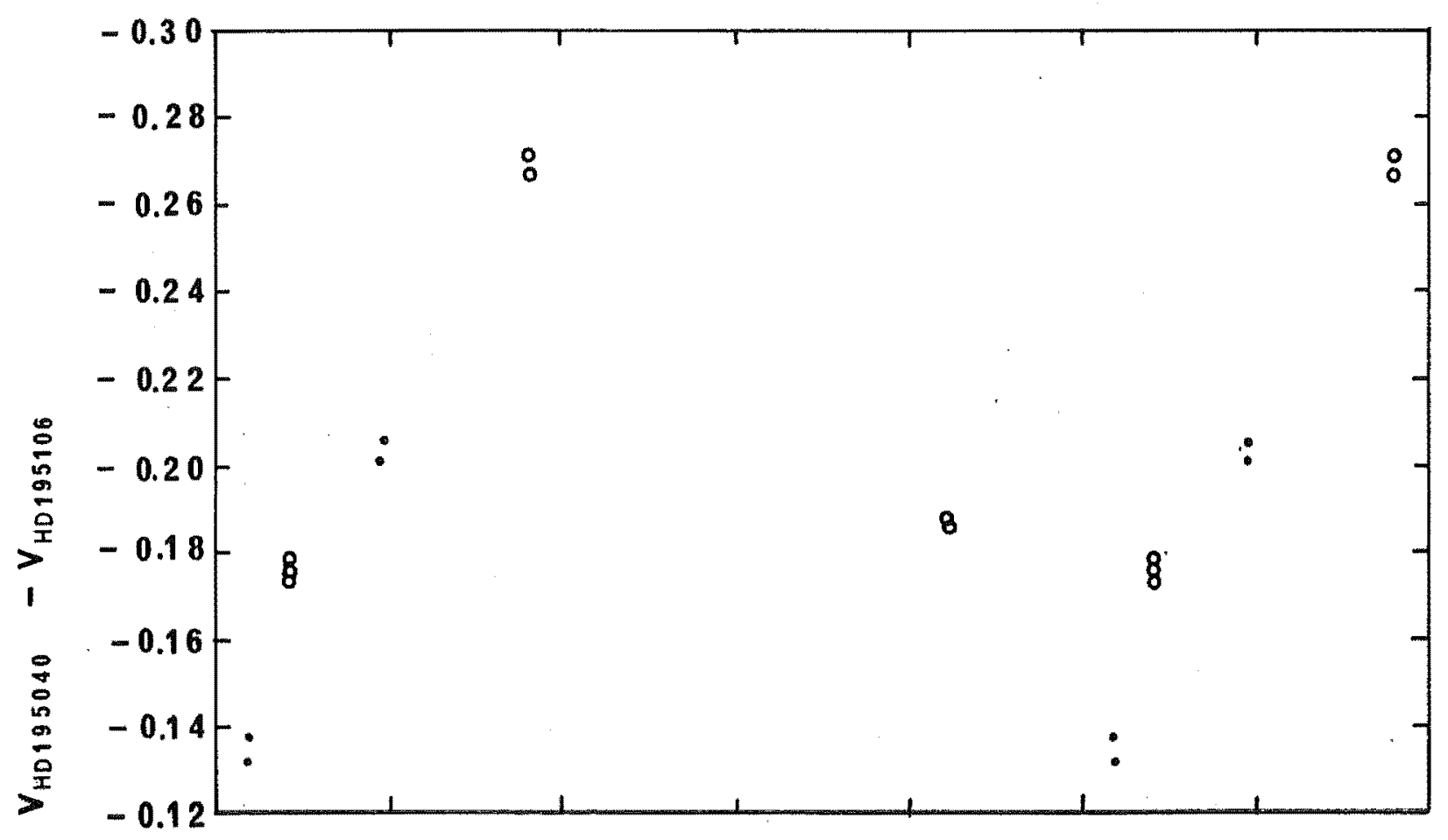

Fig. 4.22.2(a) Radial velocities of HD 195040. Symbols defined as in Fig. 4.11.3.

(b) V lightcurve of HD 195040, 1979 August (dots) and 1979 October (open circles).

The ephemeris used was

$$
\text { HJD }=2444559.25+8.30 \mathrm{E} \text {. }
$$


uncertainty in the orbital period renders the phase of wave minimum highly uncertain for the 1979 photometry; Fig. 4.22.2(b) is intended only to illustrate the shape of the lightcurve during 1979 August and October.

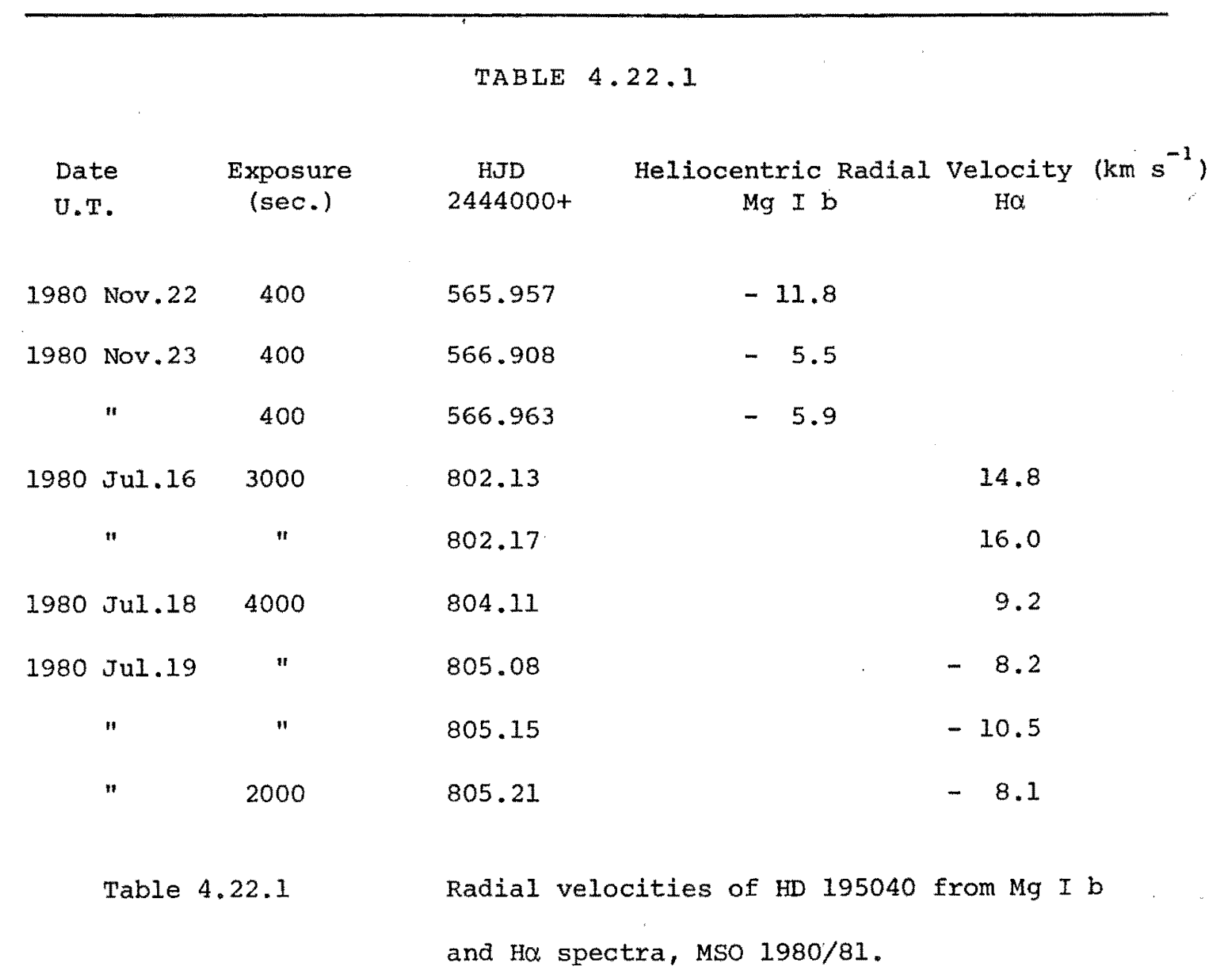


TABLE 4.22 .2

$\begin{array}{ll}\mathrm{T}_{\text {conj }} & =\text { HJD } 2444559.25 \\ \mathrm{P} & =8.30 \pm 0.03 \text { days } \\ \gamma & =1.3 \mathrm{~km} \mathrm{~s}^{-1} \\ \mathrm{~K} & =14.3 \mathrm{~km} \mathrm{~s}^{-1} \\ \mathrm{f}(\mathrm{m}) & =\end{array}$

Table $4.22 .2 \quad$ Best-fit sinusoidal radial velocity solution for HD 195040 .

$4.23 \quad$ HD 196818

Houk and Cowley (1975) classify HD 196818 as a KO IIIp object and note the presence of strong emission cores in the $\mathrm{Ca}$ II $\mathrm{H}$ and $\mathrm{K}$ lines. HD 196818 also appears on Bidelman and MacConnell's (1973) list of southern $H$ and $K$ emitters.

\subsubsection{Photometric Observations}

HD 196818 was observed on four nights during 1979 July and August. Variability was observed, with an amplitude of at least 0.02 magnitudes in $V$. The colour indices in the BVR $_{C} I_{C}$ system are listed in Table 2.1 , and agree with the Ko III spectral type given by Houk and Cowley.

\subsubsection{Radial Velocity Observations}

A spectrum of the Mg I b line region in HD 196818 was obtained at MSO on the night of 1981 October 25. The crosscorrelation function showed a single peak, with rotational broadening comparable to that seen in HD 195040. The 
heliocentric radial velocity of the c.c.f peak was $-18.5 \mathrm{~km} \mathrm{~s} \mathrm{~s}^{-1}$ at HJD 2444538.910 .

$4.23 .3 \quad \mathrm{H}_{\alpha}$ Spectra

A single spectrum of the $\mathrm{H} \alpha$ region in HD 196818 was obtained at MSO on the night of $1981 \mathrm{July}$ 16. The metallic line spectrum shows rotational broadening corresponding to $v_{e} \sin i=15 \pm 5 \mathrm{~km} \mathrm{~s}^{-1}$. The heliocentric radial velocity was $-12.2 \mathrm{~km} \mathrm{~s}^{-1}$ at HJD 2444802.25 , which suggests that the radial velocity may be variable.

The Ho profile itself (Fig. 4.23.1) shows a shallow absorption core, with weak emission features slightly above continuum level in the wings.

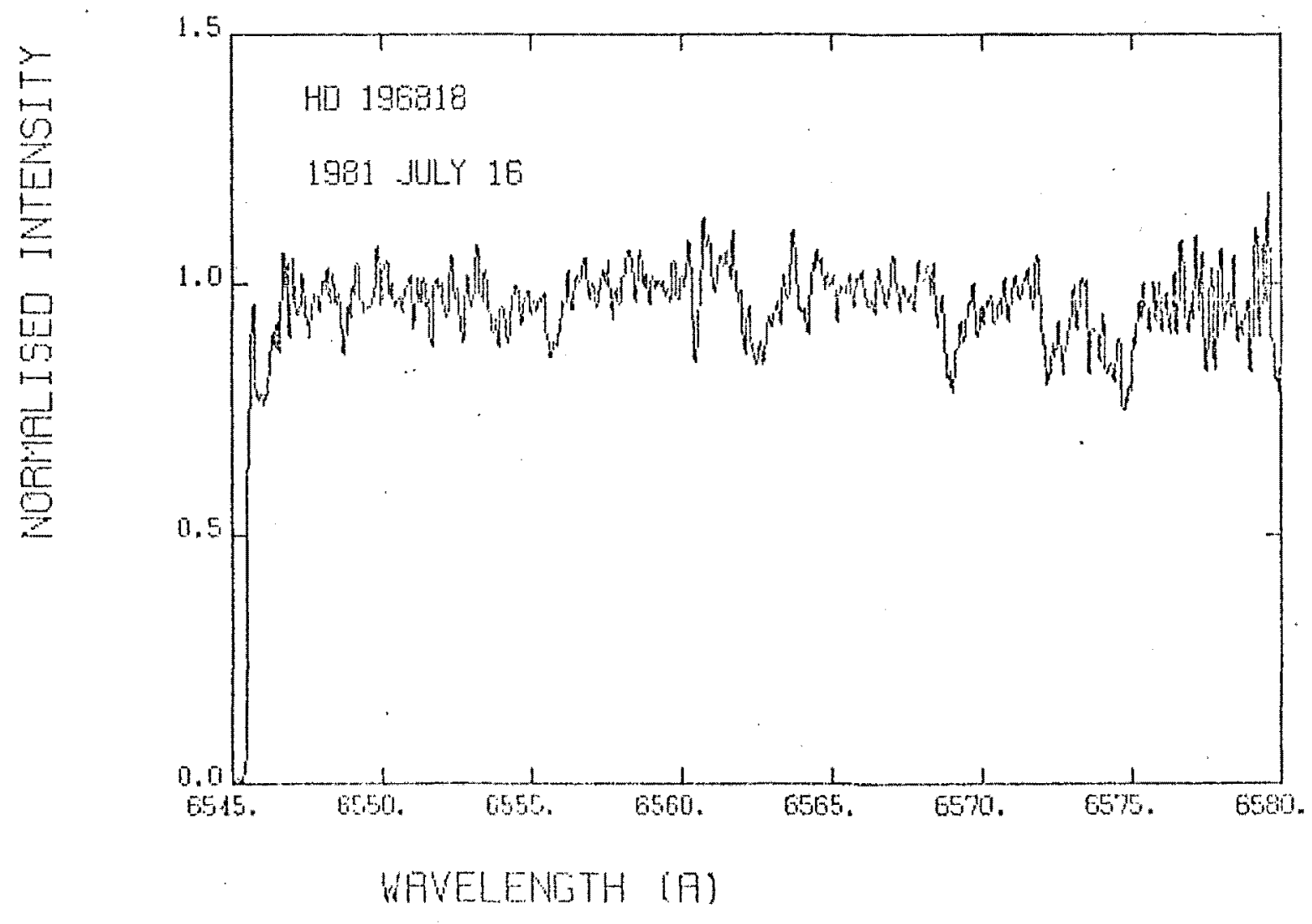

Fig. 4.23.1 H $\alpha$ spectrum of HD 196818. 


\section{SUMMARY AND DISCUSSION}

\subsection{SUMMARY OF RESULTS}

\subsubsection{Duplicity}

Extensive radial velocity measurements were obtained for 20 of the 23 stars investigated during the course of this survey. Of these twenty, only HD 32918, HD 36705 and HD 165141 showed no evidence of radial velocity variations. The remaining seventeen stars consisted of six double-lined, and eleven single-lined spectroscopic binaries.

of the three single objects, HD 32918 and HD 36705 showed extreme ( $v_{e} \sin i \geqslant 40 \mathrm{~km} \mathrm{~s}^{-1}$ ) rotational smearing of their spectra, as well as emission of $\mathrm{H \alpha}$ and rotationallymodulated broad-band photometric variability of the type observed in RS CVn systems. The natures of these two objects, which have been tentatively identified as new members of the FK Comae class of single, rapidly-rotating late-type giants with RS CVn-like surface activity, are discussed more fully by collier (1982) and in sections 4.4 and 4.6 of this thesis.

The third single star, HD 165141, has a rather lower absolute surface flux in the $\mathrm{Ca}$ II $\mathrm{H}$ and $\mathrm{K}$ line cores than the active binary components for which flux measures were obtained, and appears to be a late Hertzsprung gap object of about two solar masses.

\section{1 .2 Orbital periods}

Prior to this survey, orbital periods had been determined for HD 5303 (Collier, Hearnshaw and Austin, 1981) 
and for HD 155555 (Bennett, Evans and Laing, 1962).

From the radial velocity data presented here, orbital periods were reliably determined for HD 46697 , HD 81410, HD 101309, HR 4492 (= HD 101379+ HD 101380; both are binary systems for which periods were determined), HD 119285 and HD 127535.

The remaining systems show radial velocity variations which suggest orbital periods between about four and fifty days. In these systems, tidal interaction between the binary components is likely to have achieved at least partially synchronous axial rotation in the convective envelope of the more evolved star as will be discussed in Section 5.2.2.

The existence of RS CVn-like surface activity in the single, fast-rotating objects HD 32918 and HD 36705 provides strong evidence that the origin of the RS CVn phenomenon lies not directly in the binary nature of these stars but in their fast axial rotation.

\subsubsection{Photometric Variability}

Extensive photometric observations were obtained of sixteen of the twenty-three candidate stars. Only one, HD 5303, was found to be an eclipsing R.S CVn binary. Eclipses were also discovered in HD 101380, which is the visual companion to the giant RS CVn-like system HD 101379 $(=\operatorname{HR}$ 4492).

With only one exception, photometric variations with amplitudes between 0.02 and $0.32 \mathrm{mag}$. were observed in all the photometric program stars. In all cases where a period could be determined, the variation was regular and quasisinusoidal, indicating that an RS CVn-type photometric wave was present. 
HD 181809 showed no sign of variability with an

amplitude of more than $0.01 \mathrm{mag}$. However, the low amplitude of the radial velocity variation in this star suggests that the system (which appears to have an orbital period of the order of seven days) is viewed almost pole-on. In such a case, large amplitude rotational variability due to a nonuniform longitudinal surface brightness distribution would not be seen.

Analysis of the photometric colours of the program stars in the Johnson UBV and Cousins BVR $I_{C}$ systems revealed that the double-lined spectroscopic binaries all contained at least one component of the spectral type ko III/IV or later; the single-lined systems all had similar late spectral types.

$\underline{5.1 .4 \quad \mathrm{Ca} \text { II } \mathrm{H} \text { and } \mathrm{K} \text { Emission }}$

The most important selection criterion for the program stars was the apparent strength of the $\mathrm{Ca}$ II $\mathrm{H}$ and $\mathrm{K}$ emission features on objective prism surveys. The sample of stars examined in this survey thus contains many of the strongest $G$ and $K$ type $C a$ II emitters brighter than $V \simeq 8.5$ in the southern hemisphere.

High-resolution spectra of the $\mathrm{Ca}$ II $\mathrm{H}$ and $\mathrm{K}$ line region were obtained for nine stars, using the coudé spectrograph on the $1.88 \mathrm{~m}$ telescope at MSO in the configuration described in section 3.2.3. Typical examples of these spectra are illustrated in Fig. 5.1.1.

The absolute surface fluxes derived from these spectra using the calibration technique developed by Linsky et.al. (1979) are tabulated in Table 3.4, and show a wide range of values. 


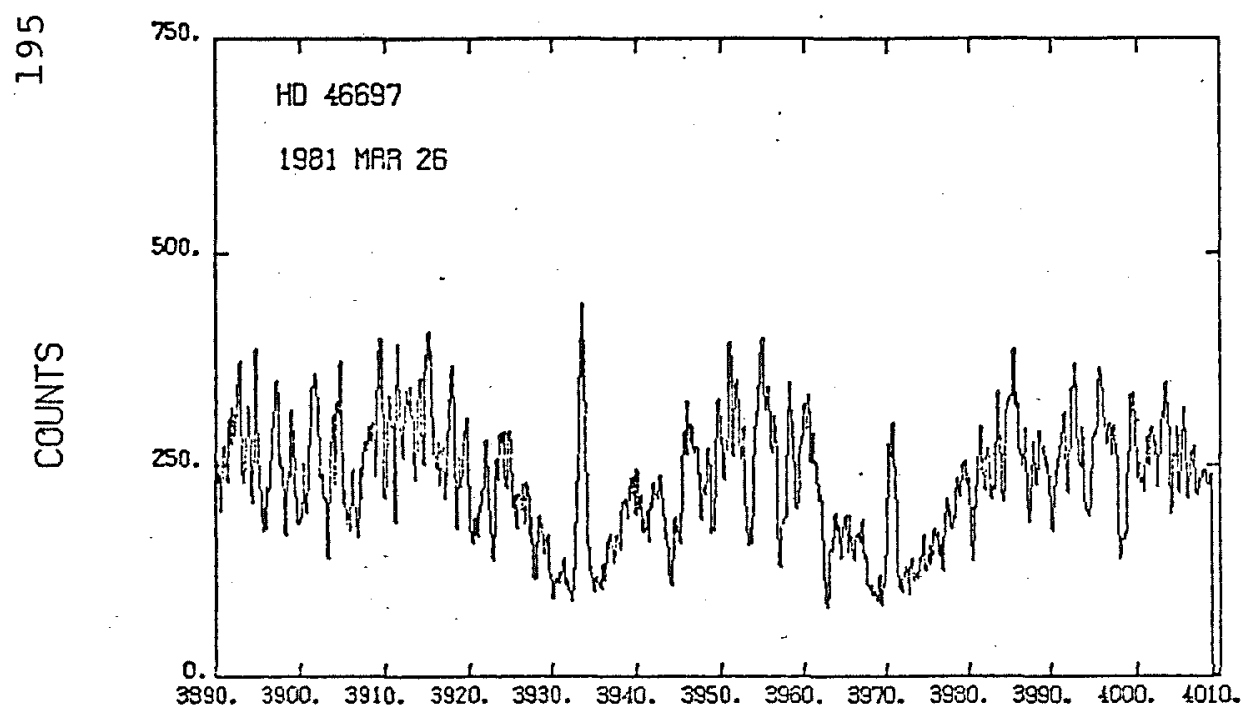

HAVELENGTH(A)

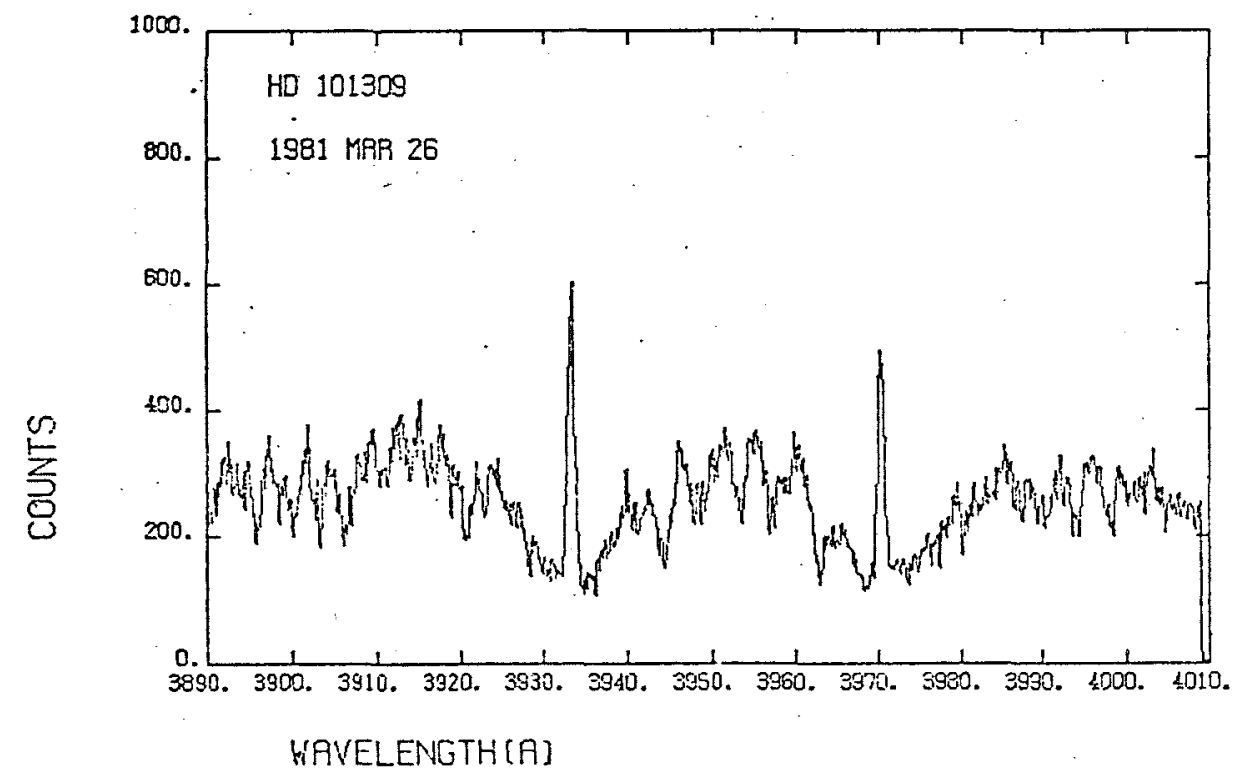

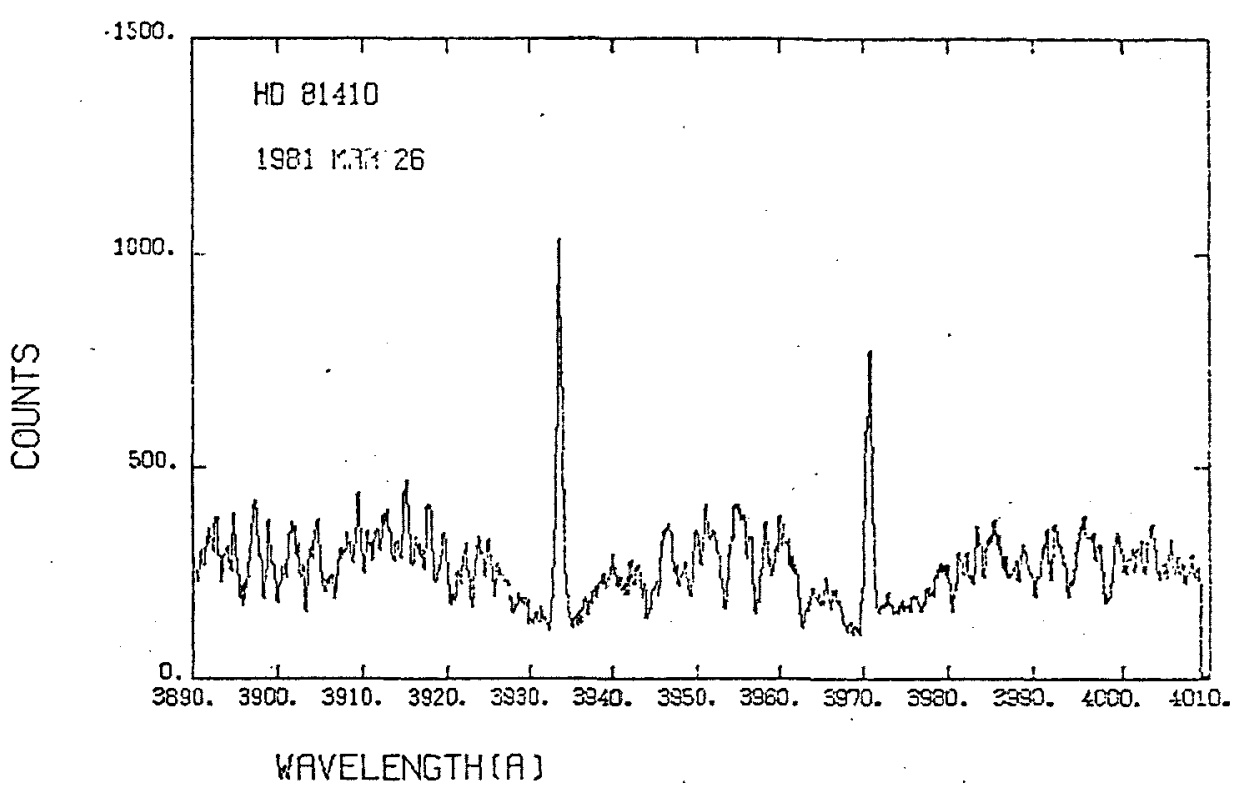

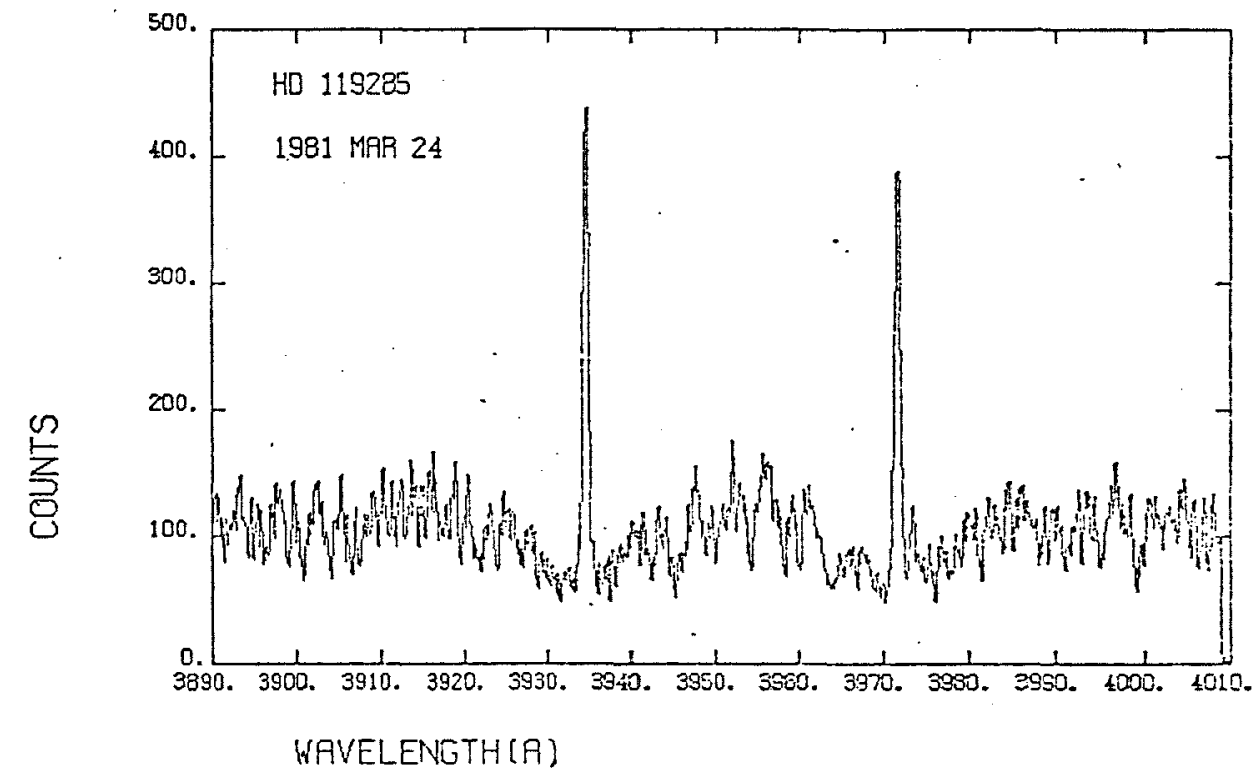

Fig. 5.1.1 Ca II $\mathrm{H}$ and $\mathrm{K}$ spectra for eight stars. 


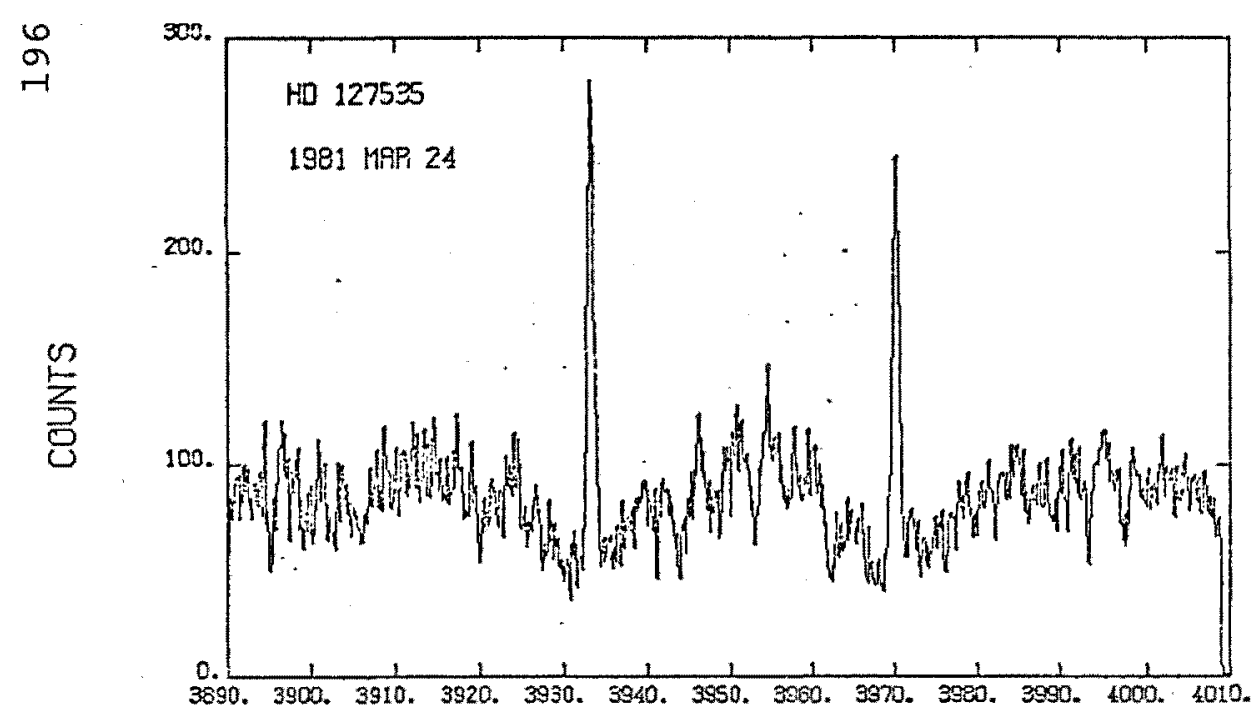

WAVELENGTH(A)

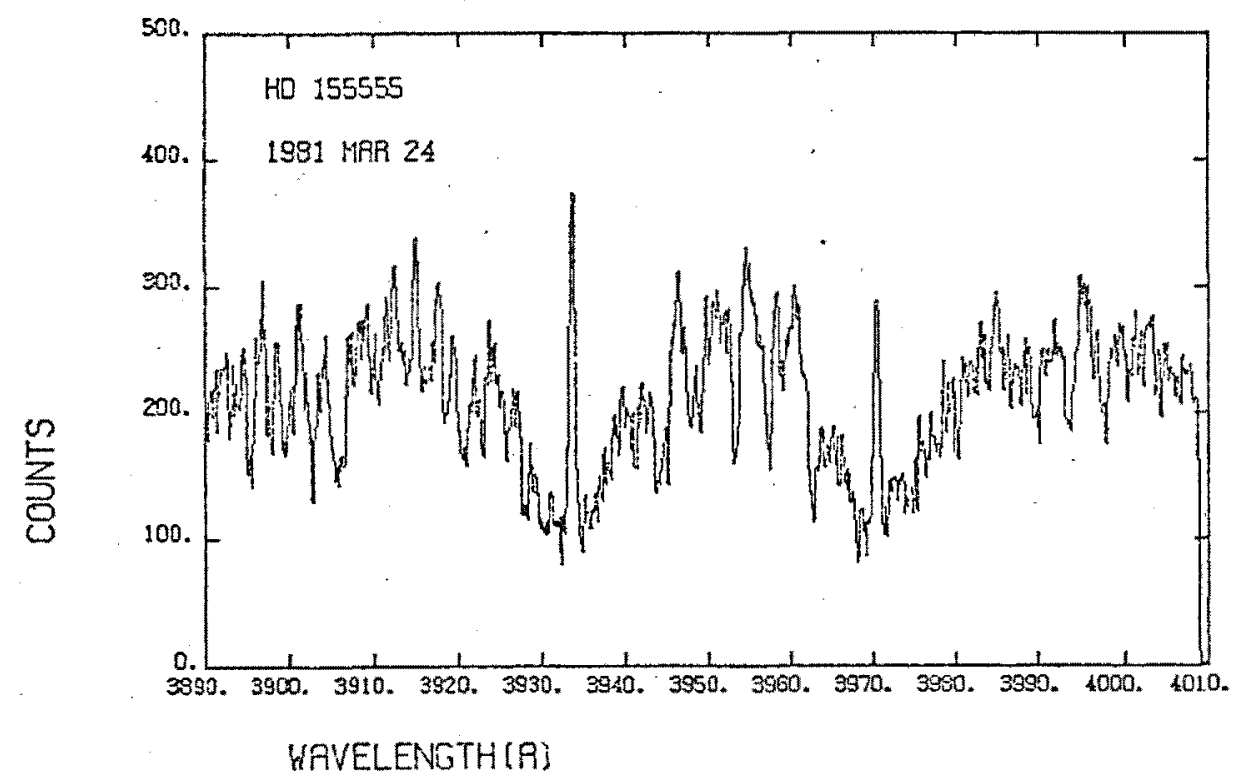

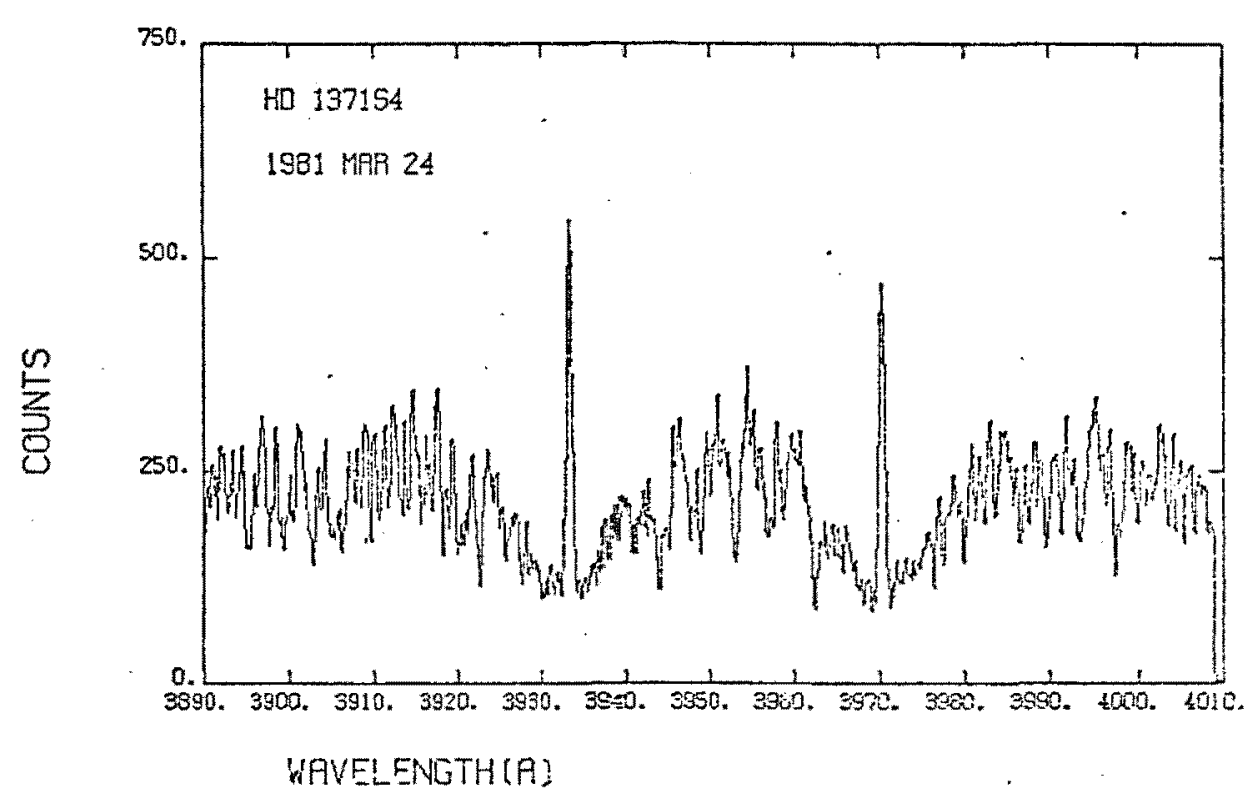

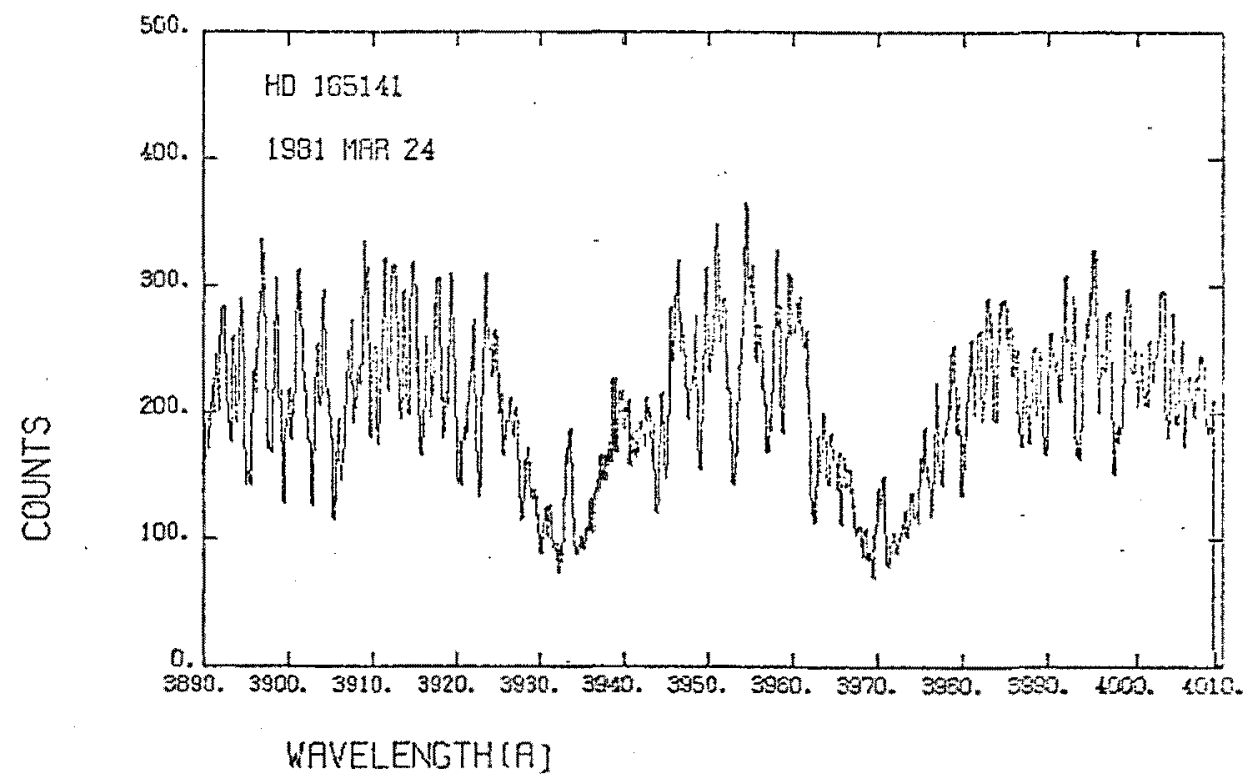

Fig. 5.1.1 (continued). 


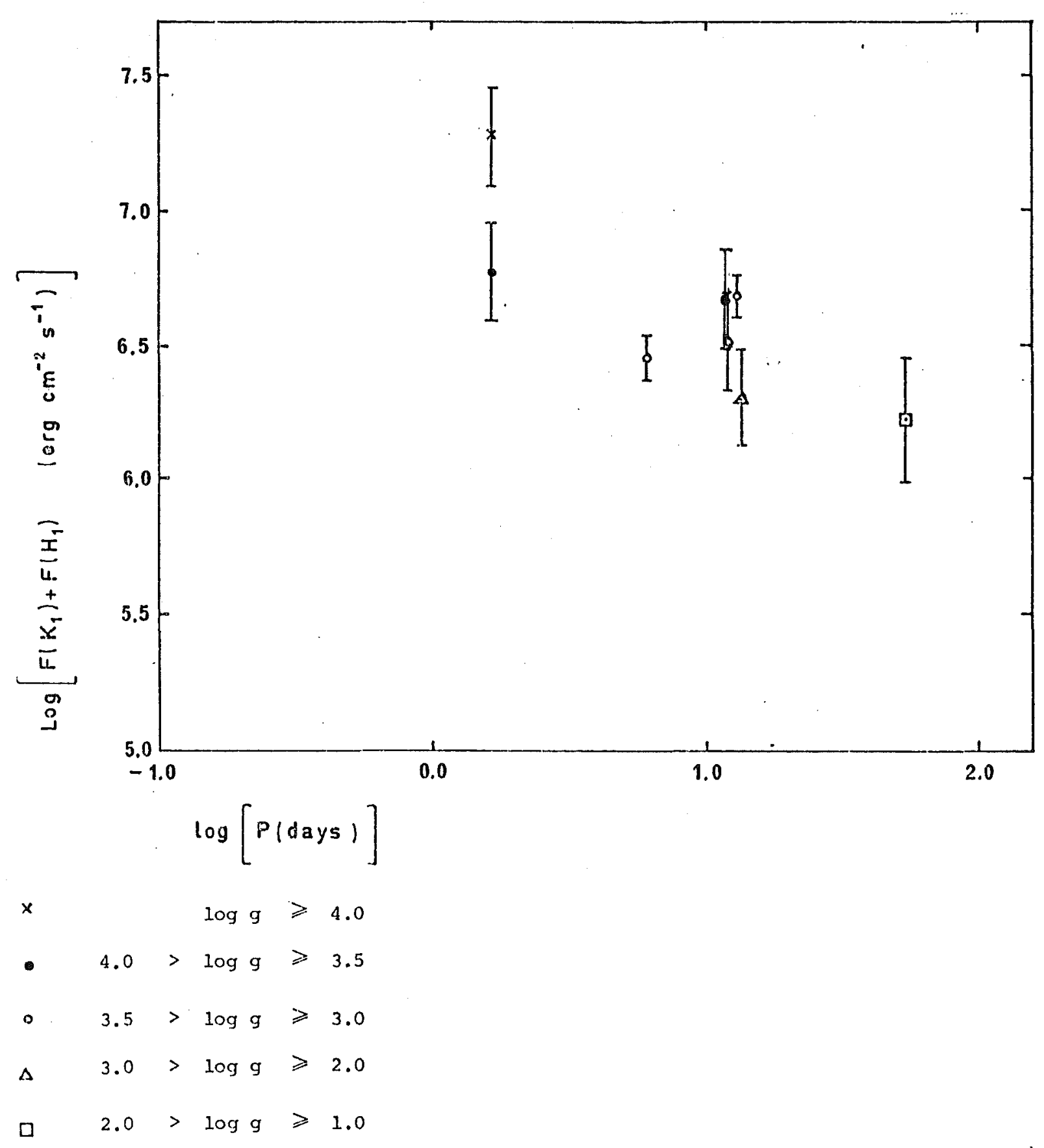

Fig. 5.1.2 Ca II $H$ and $K$ absolute line surface vs, orbital period for eight stars. Symbols denote Wilson-Bappu surface gravities as listed above. 
The surface fluxes are plotted against rotation period in Fig. 5.1.2, for those stars whose rotation periods and absolute Ca II surface fluxes were obtained. The uncertainties are large, and the sample size is small. A correlation between rotation rate and emission flux appears to be present. The fastest rotator, HD 155555, is the strongest emitter in both components; while the slowest rotator, HR 4492 (= HD 101379) is the weakest emitter.

With the exceptions of HR 4492 and the hotter component of HD 155555, the surface gravities of the stars plotted in Fig. 5.1 .2 all lie in the range $2.6 \leqslant \log \mathrm{g} \leqslant 3.6$, as calculated from the Wilson-Bappu $\mathrm{K}$ line core widths or (e.g. HD 155555) from the photometric models discussed for individual stars in Chapter 4. The Wilson-Bappu gravities are reasonably consistent with the masses and radii estimated by other means (e.g. HD 101309 and HR 4492, Chapter 4) which Iends support to Ayres' (1979) theoretical result that the FWHM of the Ca II emission core should be effectively independent of the emission flux.

The likely dependence of the surface activity levels in RS CVn stars on internal structure and differential rotation has been discussed in Chapter 1 . The range of masses and surface gravities found for the various stars discussed in Chapter 4 suggests that the internal structures of the stars plotted in Fig. 5.1.2 may differ considerably. It is interesting, however, to note that HD 81410, HD 101309 and HD 119285 appear to be intrinsically similar objects with similar rotation periods. When the intrinsic variability of the Ca II emission flux in these stars is taken into account, the absolute surface fluxes of these stars resemble each other very closely, as do their $\mathrm{H} \alpha$ profiles (Chapter 4 and Section 5.1.6). 
This result is encouraging in that it suggests that measurements of absolute surface Ca II emission flux using the calibration method of Linsky et.al. (1979) will provide a useful observational quantity to compare with future theoretical models. Relative flux measures such as the Wilson flux index $S$ are of little use in the study of double-lined spectroscopic binaires, as the apparent intensity of the Ca II emission can be highly misleading when compared to the mean level of the surrounding absorption spectrum, which depends largely on the effective temperature.

\subsubsection{Ha Emission}

High resolution $\mathrm{H} \alpha$ spectra were obtained of all the candidate stars except HD 34802 and HD 185510.

Six of these stars showed apparently normal $\mathrm{H} \alpha$ absorption profiles, accompanied (with the exception of HD 165141) by radial velocity variations and rotational smearing with $10 \mathrm{~km} \mathrm{~s}^{-1} \leqslant \mathrm{v}_{\mathrm{e}} \sin i \leqslant 25 \mathrm{~km} \mathrm{~s}^{-1}$.

Six stars showed $\mathrm{H} \alpha$ as either an unusually weak absorption feature or else completely absent (presumably. filled with emission to continuum level), while the remaining nine all displayed $H \alpha$ emission features above continuum level. In some cases where strong emission was present, a weak, narrow absorption core was present at the wavelength of the line centre.

In those stars in which $\mathrm{H} \alpha$ emission was present, the emission profile was seen to undergo erratic variations in shape and intensity. The emission profile was generally of the order of $200 \mathrm{~km} \mathrm{~s}^{-1}$ in width, and in many cases appeared displaced, towards the blue by up to $100 \mathrm{~km} \mathrm{~s}^{-1}$. Similar blue-shifted $\mathrm{H} \alpha$ emission features have been observed in 
several northern hemisphere RS CVn binaries (Bopp and, Talcott, 1980). The erratic variability, velocity dispersion and blue-shift of the emission are consistent with the mechanism proposed by Bopp and Talcott, i.e. prominence activity triggered by sporadic surface flare activity. The simultaneous $H \alpha$ emission enhancements and radio flares observed in HR 1099 (Bopp and Talcott, 1978; Hearnshaw, 1978; Weiler et.al., 1978) and in HR 4492 (Collier et.al., 1982) lend support to this hypothesis for the origin of the $\mathrm{H} \alpha$ emission.

of the six stars in which $H \alpha$ appeared as a more or less normal absorption feature, only HD 14643 was observed photometrically. An RS CVn-type variation with an amplitude of more than $0.15 \mathrm{mag}$. was observed.

All the $\mathrm{H} \alpha$ emitters for which photometry was abtained also showed significant photometric variability, including the two new FK Comae objects HD 32918 and HD 36705.

Bopp et.al. (1981) have proposed that the presence of $H \alpha$ emission in the spectra of $d K$ and $d M$ stars is a sufficient, but not a necessary, condition for the onset of the BY Draconis syndrome. It appears from this and other surveys (Bopp and Talcott, 1978, 1980) that a similar result may hold for late $G$ and early $K$ giants and subgiants, such as the FK Com stars and the active components of RS CVn binaries. In spite of the sporadic variations seen in the $\mathrm{H} \alpha$ emission profiles of these stars, there appears to be a wide range of mean quiescent emission levels. The most active, such as HR 1099, show Ha permanently in emission, whereas others have mean levels which result only in a partial filling of the $H \alpha$ absorption profile. This is partly a contrast effect. The apparent strength of the Ha emission 
depends on

(a) the absolute $H \alpha$ emission surface flux;

(b) the surface brightness of the background photospheric spectrum from the $\mathrm{H} \alpha$ emitting star;

(c) the contribution of a secondary component's photospheric spectrum at $\mathrm{H} \alpha$ in a double-lined spectroscopic binary.

The problem is similar to that encountered in evaluating the $\mathrm{Ca}$ II $\mathrm{H}$ and $\mathrm{K}$ emission flux in absolute surface flux units. For instance, by applying the model atmosphere grid of Kurucz to the cool components in HD 155555 and $H D 81410$ and correcting for duplicity, we find that the continuum surface flux at $H \alpha$ in $H D 155555$ is about three times that in HD 81410 . The apparent Ho emission strengths in these two stars are more or less equal; the absolute surface emission flux in HD 155555 is, however, higher by a factor of about three.

None of the stars showing $\mathrm{H} \alpha$ as a normal absorption feature has a rotation period of less than 14 days. From the rough estimates made here, it appears that the short period objects such as HD 5303, HR 1099 and HD 155555 also have the highest quiescent Ho emission levels.

\subsection{SELECTION EFFECTS}

\subsubsection{Observed Sample Properties for Different Detection}

Methods

One of the main criteria in the selection of the candidate stars for this study was the strength of the Ca II emission on objective prism surveys by Bidelman and Macconnell (1973), Houk and Cowley (1975) and Houk (1978). The 
strongest $H$ and $K$ emitters with $G-K$ spectral types and $\mathrm{V} \leqslant 8.5$ were given the highest priority.

There are substantial differences between the physical characteristics of the sample of stars studied here and the majority of the known RS CVn systems in the northern hemisphere.

The northern systems are predominantly double-lined binaries with relatively weak $\mathrm{Ca}$ II $\mathrm{H}$ and $\mathrm{K}$ emission features. Popper and Ulrich (1977) list 26 RS CVn binaries, of which 23 are eclipsing. It should be remembered that the RS CVn binaries originally attracted attention as a class not because of their chromospheric activity, but because of their photometric peculiarities as eclipsing binaries.

Although a large number of non-eclipsing RS CVn systems has since been identified in the northern hemisphere, the northern sample is still very heavily biased in favour of the eclipsing and double-lined systems.

On the other hand, the southern stars selected for the present study on the basis of their Ca II emission strengths show rather different general properties. If we exclude 'HD 155555 (which was selected for different reasons), we find that of sixteen newly-identicied spectroscopic binaries with strong Ca II emission, only HD 5303, HD 8435, HD 37847, HD 101309, HD 137164 and possibly HD 14643 exhibit double-lined spectra. For the whole sample, the spectral types, masses and radii of the active components, the surface activity levels and the orbital periods are similar to those seen in the northern RS CVn systems. However, the absence of an observable secondary spectrum in most cases suggests that the use of the Ca II emission strength as a selection criterion favours the discovery of non-eclipsing, single- 
Iined systems.

\section{2 .2 Effects of Secondary Luminosity on Detection Likelihood}

The apparent strength of the $\mathrm{Ca}$ II emission on an objective prism spectrum is largely dependent on the relative strength of the photospheric spectrum around $3950 \AA$.

The common feature shared by nearly all the northern and southern RS CVn systems is that the more massive component (hereinafter referred to as the primary) seems to have evolved to a point near the base of the giant branch on the evolutionary track for the appropriate mass, which lies in the range $1.0 \leqslant \frac{\mathrm{M}_{1}}{\mathrm{M}_{\odot}} \leqslant 2.0$. In doing so, it has remained within its Roche lobe, and no rapid mass exchange due to Roche lobe overflow has yet taken place. The mass ratio of the system is thus presumably close to its initial value, although some mass loss from the primary may have taken place due to enhanced mass outflow from regions of strong surface activity.

A secondary component of only slightly lower mass than the primary will have a higher effective temperature than, and comparable luminosity to, the primary. The secondary's light will thus dominate the blue end of the spectrum. The apparent Ca II emission strength on objective prism plates will be reduced by a factor depending on the relative luminosities of the components around $3950 \AA$. However, if eclipses occur, the high surface brightness ratio of the components will result in a deep, easily detectable eclipse when the hotter star is eclipsed.

The effects of reducing the mass of the secondary component on the apparent $\mathrm{Ca}$ II emission strength and the eclipse depth are interesting, and are calculated below for 
a"typical" system whose primary has a mass of $1.3 \mathrm{M}_{\odot}$ and has evolved to the base of the giant branch.

The age of the system is taken as $4 \times 10^{9}$ years, and the temperature and bolometric luminosity of the secondary" component is derived as a function of mass using isochrones calculated by Sandage and Eggen (1969) from Iben's (1967) evolutionary tracks for stars with initial composition $x=0.709, \quad y=0.272, \quad z=0.020$ (Fig. 5.2.1).

The parameters obtained for the primary are $T_{\text {eff }}=4800 \mathrm{~K}, \mathrm{M}_{\mathrm{bol}}=2.9, \mathrm{R}=3.4 \mathrm{R}_{\mathrm{O}}$. The $(\mathrm{V}-\mathrm{R})_{\mathrm{J}}$ colour index obtained from $T_{\text {eff }}$ and the mean relations of Johnson (1966) is 0.81 . The calibration of Linsky et.al. (1979; see Section 3.5.5) then yields an absolute photospheric surface flux (in erg cm $\mathrm{cm}^{-1} \mathrm{~A}^{-1}$ ) in the band 3925-3975 $A$, $\log F(\Delta \lambda)=5.77$.

The total luminosity of the system integrated over the 3925-3975 $\AA$ band is

$$
L_{\text {sys }}(\Delta \lambda) \sim R_{1}{ }^{2} \Delta \lambda F_{1}(\Delta \lambda)+R_{2}{ }^{2} \Delta \lambda F_{2}(\Delta \lambda)
$$

or, in units of the primary's Iuminosity in this band

$$
\frac{L_{\text {sys }}(\Delta \lambda)}{R_{1}{ }^{2} \Delta \lambda F_{1}(\Delta \lambda)}=1+\frac{R_{2}^{2}}{R_{1}^{2}} \frac{F_{2}(\Delta \lambda)}{F_{1}(\Delta \lambda)} \text {. }
$$

This is the factor by which the apparent strength of the $\mathrm{Ca}$ II emission will be reduced on an objective prism plate, due to the secondary's contribution to the background flux near the $\mathrm{H}$ and $\mathrm{K}$ lines.

In all the models, the primary has a greater radius than the less massive secondary. Approximate maximum eclipse depths in the $\mathrm{V}$ band are calculated for orbital inclination $i=90^{\circ}$, and are listed in the last two columns of Table 5.2 .1 , using surface brightnesses $F(\lambda, T)$ interpolated for 
$\lambda=5500 \AA$ from the model atmosphere grid of Kurusz.

A simple model using spherical stars of uniform surface brightness (no Iimb darkening) gives

$$
\begin{aligned}
& \Delta V_{\text {OC }}=-2.5 \log \left[\frac{1}{1+\frac{R_{2}{ }^{2}}{R_{1}{ }^{2}} \frac{F\left(5500, \mathrm{~T}_{2}\right)}{F\left(5500, \mathrm{~T}_{1}\right)}}\right] \\
& \left.\Delta V_{\mathrm{tr}}=-2.5 \log \left[\frac{\left.1+\frac{\mathrm{R}_{2}{ }^{2}}{\mathrm{R}_{1}{ }^{2}\left(\frac{F\left(5500, \mathrm{~T}_{2}\right)}{F\left(5500, \mathrm{~T}_{1}\right)}\right.}-1\right)}{\frac{\mathrm{R}_{2}{ }^{2}}{\frac{\mathrm{R}_{1}{ }^{2}}{F\left(5500, \mathrm{~T}_{2}\right)}}}\right]\right]
\end{aligned}
$$

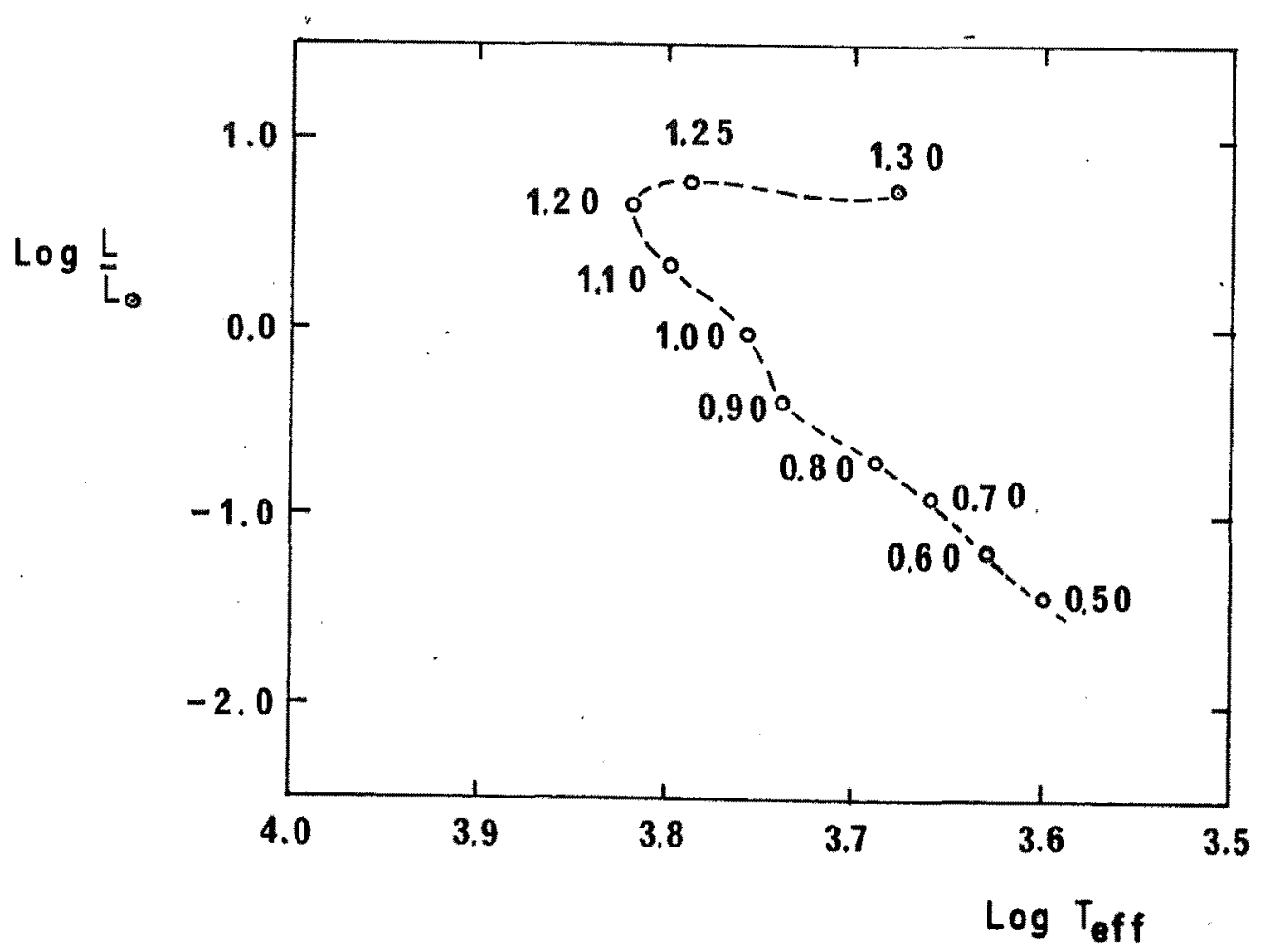

Fig. 5.2.1 Isochrone for $t=4 \times 10^{9}$ years, from Sandage and Eggen (1969). Composition is $x=0.708, y=0.272, z=0.020$ (Iben, 1967). The points along the isochrone are labelled with the masses of the corresponding models. 
TABLE 5.2 .1

$\frac{M_{2}}{M_{\odot}} \quad T_{\text {eff }} \quad M_{b_{2} I_{2}} \quad \frac{R_{2}}{R_{\odot}} \quad\left(V-R_{J}\right)_{2} \quad \log _{2}(\Delta \lambda) \quad \frac{F_{\text {sys }}(\Delta \lambda)}{R_{1} F_{1}(\Delta \lambda)} \quad \frac{F\left(5500, T_{2}\right)}{F\left(5500, T_{1}\right)} \quad M_{v_{2}}-M_{V_{1}} \quad$ (occ.) $\quad$ (trans.)

$\begin{array}{lllllllllll}1.30 & 4800 & 2.9 & 3.40 & 0.81 & 5.77 & 2.00 & 1.00 & 0.00 & 0.75 & 0.75 \\ 1.25 & 6200 & 2.8 & 2.14 & 0.44 & 6.91 & 6.47 & 3.27 & -0.28 & 0.90 & 0.21 \\ 1.20 & 6600 & 3.1 & 1.64 & 0.37 & 7.13 & 6.33 & 4.23 & 0.02 & 0.74 & 0.14 \\ 1.10 & 6300 & 3.9 & 1.25 & 0.42 & 6.97 & 3.14 & 3.48 & 0.82 & 0.42 & 0.10 \\ 1.00 & 5800 & 4.8 & 0.97 & 0.53 & 6.63 & 1.59 & 2.42 & 1.76 & 0.20 & 0.08 \\ 0.90 & 5440 & 5.7 & 0.73 & 0.58 & 6.48 & 1.24 & 1.71 & 2.76 & 0.08 & 0.05 \\ 0.80 & 4950 & 6.5 & 0.61 & 0.74 & 5.99 & 1.05 & 1.17 & 3.56 & 0.04 & 0.03 \\ 0.70 & 4610 & 7.0 & 0.56 & 0.90 & 5.50 & 1.01 & 0.79 & 4.17 & 0.02 & 0.03 \\ 0.60 & 4300 & 7.7 & 0.46 & 1.03 & 5.10 & 1.00 & 0.44 & 5.23 & 0.01 \\ 0.50 & 4000 & 8.3 & 0.41 & 1.15 & 4.73 & 1.00 & 0.11 & 0.02 & 0.00\end{array}$

Table 5.2.1 Model system parameters for $M_{1}=1.3 M_{\odot}, t=4 \times 10^{9}$ years, and various secondary masses. 


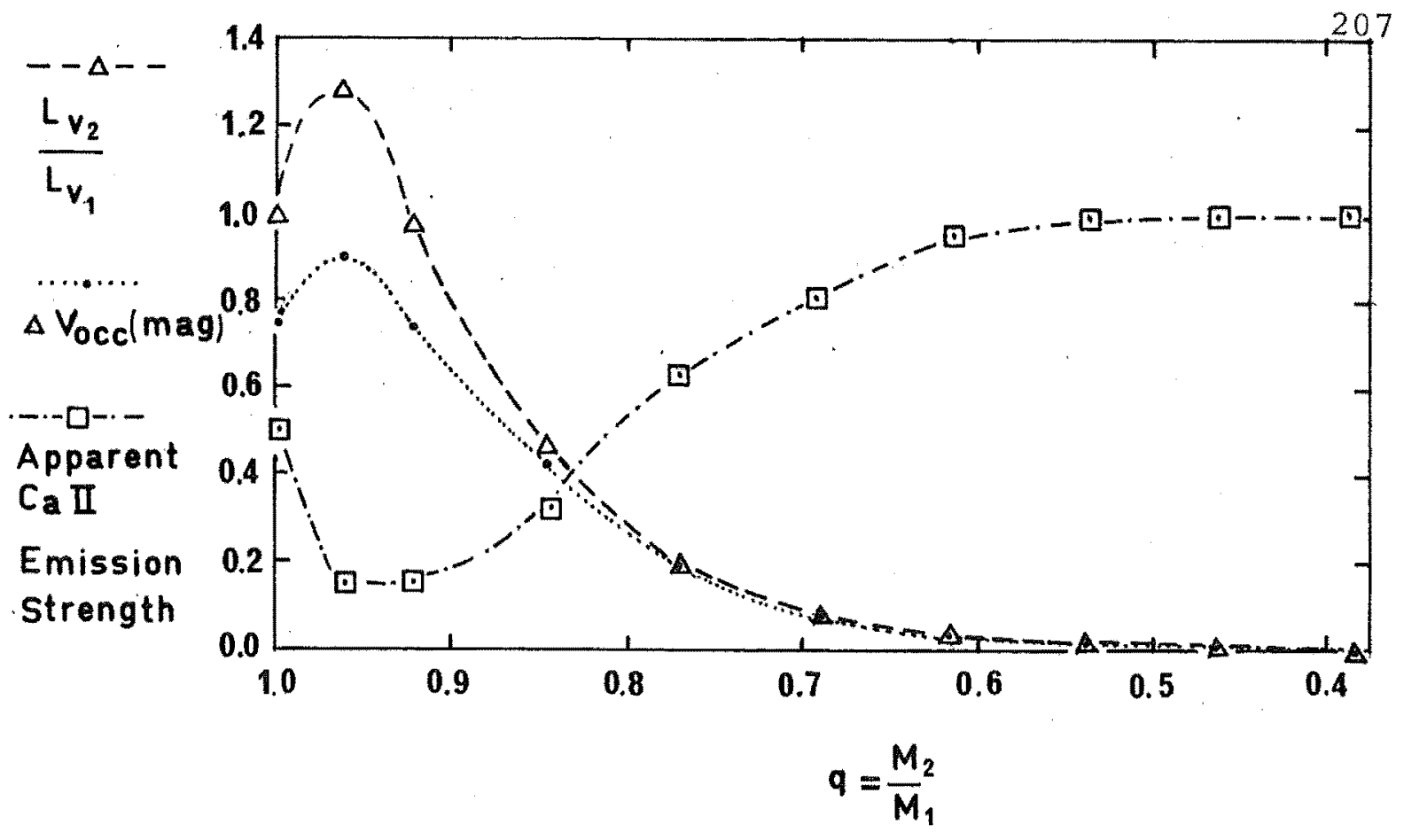

Fig. 5.2.2 Visual luminosity ratio, maximum visual eclipse depth and apparent $\mathrm{Ca}$ II emission strength, as functions of mass ratio for $M_{1}=1.3 M_{\odot}, t=4 \times 10^{9}$ years.

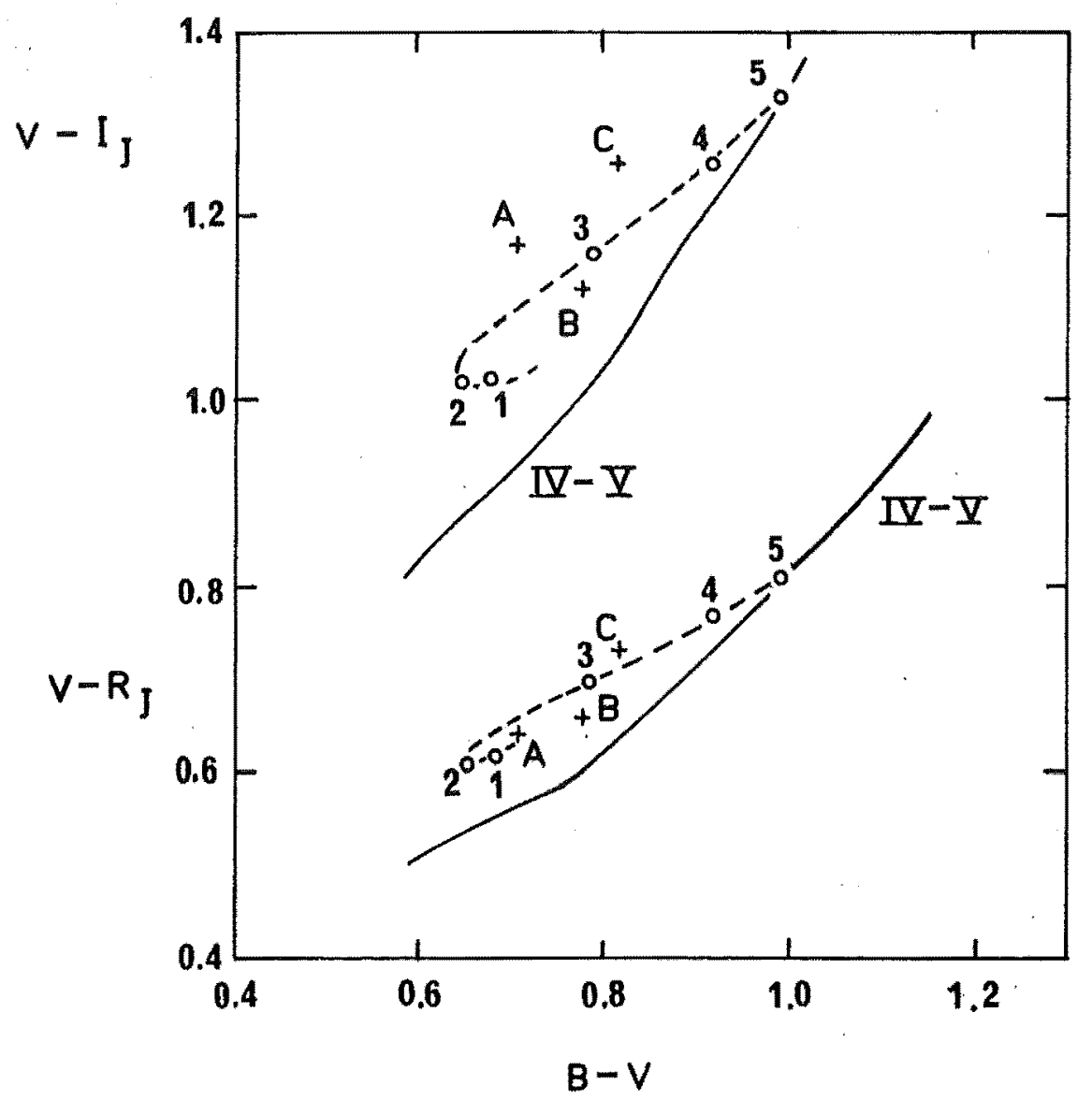

Fig. 5.2.3 Deviations of colour indices from mean Johnson relations Secondary masses at labelled points are $\mathrm{M}_{2}=$ (1) $1.2 \mathrm{M}_{\odot} ;$ (2) $1.1 \mathrm{M}_{\odot} ;$ (3) $1.0 \mathrm{M}_{\odot} ;$ (4) $0.9 \mathrm{M}_{\odot} ;(5) \leqslant 0.8 \mathrm{M}_{\odot}$ Three double-lined RS CVn systems are plotted (A) HD 5303 ; (B) HD 155555 ; (C) HD 14643. 
Fig. 5.2.2. illustrates the variation of the visual luminosity ratio $L_{v_{2}} / L_{v_{1}}$, the maximum visual eclipse depth $\Delta V_{\text {occ }}$ for complete occultation of the secondary component, and the apparent $\mathrm{Ca}$ II $\mathrm{H}$ and $\mathrm{K}$ emission strengths, normalised to the value observed when the light of the secondary is completely removed.

The observable characteristics of this model system undergo a significant change at $0.7 \leqslant q \leqslant 0.8$. Over this narrow range of secondary masses, the secondary's spectrum becomes undetectable even at blue wavelengths, the binary becomes effectively single-lined at all optical wavelengths and the eclipse depths become small. The shallowness of the eclipses renders accidental detection of the star as an eclipsing binary unlikely, but if strong Ca II emission is present in the primary it is much more likely to be detected on an objective prism survey.

The detection of non-eclipsing systems with mass ratios close to unity is perhaps the most difficult task in the search for new RS CVn systems. However, as the temperatures of the components in $q \simeq 1$ systems of this type differ considerably, the duplicity often reveals itself as a composite spectrum on an objective prism survey, or as an apparent discrepancy between the $B-V, V-R$ and $V-I$ colour indices.

For example, if we use the mean relations of Johnson (1966) to assign colour indices to the components in the model above according to their effective temperatures, we obtain $\mathrm{B}-\mathrm{V}=0.99, \mathrm{~V}-\mathrm{R}_{\mathrm{J}}=0.81$ and $\mathrm{V}-\mathrm{I}_{\mathrm{J}}=1.33$ for the primary. The results for the secondary and for the system as a whole are given in Table 5.2.2, and plotted in Fig. 5.2.3. 
TABLE 5.2 .2

\begin{tabular}{|c|c|c|c|c|c|c|c|}
\hline$M_{2}$ & $\Delta M_{V}$ & & Secondary & Colours & Syste & Colou & \\
\hline$\overline{M_{\odot}}$ & & $B-V_{J}$ & $\mathrm{~V}-\mathrm{R}_{\mathrm{J}}$ & $V-I_{J}$ & $B-v_{J}$ & $V-R_{J}$ & $V-I_{J}$ \\
\hline 1.25 & -0.28 & .49 & .44 & .70 & .68 & .62 & 1.02 \\
\hline 1.20 & 0.02 & .38 & .37 & .58 & .65 & .61 & 1.02 \\
\hline 1.10 & 0.82 & .46 & .42 & .67 & .79 & .70 & 1.16 \\
\hline 1.00 & 1.76 & .63 & .53 & .86 & .92 & .77 & 1.26 \\
\hline 0.90 & 2.76 & .74 & .58 & .96 & .97 & .79 & 1.31 \\
\hline$\leqslant 0.80$ & $\geqslant 3.56$ & $\geqslant .92$ & $\geqslant .74$ & $\geqslant 1.22$ & .99 & .81 & 1.33 \\
\hline & Table & 5.2 .2 & $\begin{array}{l}\text { System col } \\
\text { masses, fo } \\
t=4.0 \mathrm{x}\end{array}$ & $\begin{array}{l}\text { our indices } \\
10^{9} \text { years. }\end{array}$ & $\begin{array}{l}\text { for various } \\
\mathrm{M}_{1}=1.3 \mathrm{M}_{\odot}\end{array}$ & secondary & \\
\hline
\end{tabular}

The deviations of the system colours from the mean Johnson relations are very similar to those observed for HD 5303, HD 14643 and HD 155555 (Table 2.3 and Fig. 2.1). Both HD 5303 and HD 155555 are double-lined RS CVn systems. HD 14643 is a binary with only a single spectrum visible at $\mathrm{H} \alpha$ and the $\mathrm{Mg} \mathrm{I} \mathrm{b}$ lines. However, the colour indices suggest that the secondary's light contributes significantly to the system's luminosity at blue wavelengths.

All the other stars plotted in Fig. 2.1 are singlespectrum objects. The difference between the single-lined Ca II emitters and the double-lined ones shows up clearly; the single-lined objects are redder and lie closer to the mean colour relations for dwarf and subgiant stars than their double-lined counterparts. This distinction may provide a 
useful means of selecting likely candidates in future searches for new double-lined RS CVn binaries.

5.3 EVOLUTIONARY CONSTRAINTS ON THE OBSERVED CHARACTERISTICS

OF RS CVn SYSTEMS

\subsubsection{Distribution of Initial Mass Ratios}

Lucy and Ricco (1979) have performed an extensive statistical investigation of the distribution of mass ratios among 178 double-lined, detached spectroscopic binaries. The distribution was found to have a narrow peak at $q \simeq 0.97$. Various selection effects were investigated, none of which explained satisfactorily this peak, which Lucy and Ricco concluded was a by-product of the binary formation mechanism.

The mass ratio distribution of double-lined RS CVn binaries was found to exhibit a similar drop in probability density for $q \leqslant 0.95$. This is not explainable in terms of the selection effects already discussed, governing the detection of the secondary component. This might be expected to cause a drop in probability density at $q \simeq 0.8$, but not at $q \simeq 0.95$. It seems likely that some aspect of the binary formation mechanism favours formation of binaries with $q \simeq 1$, but does not preclude the formation of binaries with considerably lower mass ratios.

Indeed, the high proportion of single-lined RS CVnlike binaries discovered both in this investigation and in recent northern hemisphere surveys indicates that the total space density of such $q \leqslant 0.8$ binaries is at least equal to that of the "classical" double-lined RS CVn binaries. 
5.3.2 Necessary Conditions for the RS CVn Syndrome

The principal requirements for the RS CVn phenomenon in a close binary are assumed for the purposes of this discussion to be

(1) that one or both components should posses convective envelopes, and

(2) that tidal interaction should have induced an axial rotation rate in the convective component(s) which is significantly greater than that expected for a single star of similar mass, age and composition.

Both of these conditions are based on the assumption that the source of the surface magnetic activity is a hydromagnetic dynamo of the aw type operating at the base of the star's convective envelope. This assumption seems reasonable, in view of the observational evidence discussed in Chapter 1, and leads immediately to condition (1) above.

The second condition is based on the empirical observation that the strongest Ca II emitters with G/K III/IV spectral types observed to date all belong to close binary systems with periods of the order of 50 days or less. The only exceptions are the single FK Comae stars, whose rapid axial rotation rates may have come about through the coalescence of WuMa systems (Webbink, 1976; Bopp and Stencel, 1981; Collier, 1982) during the course of their post-main-sequence evolution.

Moreover, the Ca II emitters with the highest absolute surface emission fluxes in the $H$ and $K$ line cores found during the course of this survey all appear to be stars of between one and two solar masses which have evolved to the base of the giant branch. At this stage in their evolution, 
these stars possess deep convective envelopes, whose outer radii have expanded to between three and five times the star's initial main sequence radii. In order to conserve angular momentum, a single star's rotation rate decreases greatly during its Hertzsprung gap evolution.

The presence of a close binary companion, however, may result in a substantially greater rotation rate than would be expected for a single Hertzsprung gap object, or even for a late main sequence star, resulting in an enhanced level of surface magnetic activity.

Conditions (1) and (2) above may be fulfilled when one or both binary components is either on the lower main sequence, in the late stages of Hertzsprung gap evolution, or on the ascending giant branch during shell hydrogen burning and prior to the onset of core helium burning.

In all three cases, the stars' radii and separation must be such that synchronous axial rotation can be achieved before the more massive star overflows its Roche lobe and mass exhange takes place. For the remainder of this discussion, an "RS CVn system" will be defined as a detached binary which satisfies both the above conditions while the primary is crossing the Hertzsprung gap.

\subsubsection{Period Constraints on RS CVn Binary Systems}

If the more massive (primary) component of a binary is to complete its Hertzsprung gap evolution while remaining detached, the separation of the components must be such that the mean radius of the primary's Roche lobe is greater than the primary's radius at the base of the giant branch. Paczynski (1971) gives an approximate expression for the Roche lobe radius $R_{L}$ of the more massive star in a close binary 


$$
\begin{aligned}
\frac{\mathrm{R}_{\mathrm{L}}}{\mathrm{a}} \approx 0.38- & 0.2 \log \mathrm{q} \\
& \text { where a is the mean orbital } \\
& \text { separation of the stars, } \\
& \text { and } \mathrm{q}=\frac{\mathrm{M}_{2}}{\mathrm{M}_{1}} \text { is the mass } \\
& \text { ratio. }
\end{aligned}
$$

If we substitute this into the usual expression for Keplerian motion, we find that the minimum orbital period for the above condition to be satisfied is

$$
\begin{aligned}
P_{\min }=\left[\frac{R_{1}{ }^{3}}{M_{1}(1+q)(0.38-0.2 \cdot \log q)^{3}}\right]^{\frac{1}{2}} \\
\text { where } P_{\text {min }} \text { is expressed } \\
\text { in years, the radius of } \\
\text { the primary } R_{1} \text { in a.u. } \\
\text { and the primary mass } M_{1} \\
\text { in } M_{\odot} .
\end{aligned}
$$

For this and the remaining evolutionary calculations in this chapter, the set of evolutionary tracks published by Mengel et al. (1980) for stars of composition $\mathrm{X}=0.79$, $z=0.01$, will be used. These models follow the evolution of stars with masses betwen 0.7 and $4.4 \mathrm{M}_{\odot}$ from the zero-age main sequence to the end of the subgiant phase at the base of the giant branch. The models for stars with masses $0.9,1.1$, $1.4,1.75$ and $2.2 \mathrm{M}_{\odot}$ are extended from the subgiant phase to the start of core helium burning at the tip of the giant branch, by Sweigart and Gross (1978). All quantities such as luminosities, surface gravities and timescales have been derived from these models.

Table 5.3.1 gives the radii for stars of various masses at the base of the giant branch, with the corresponding minimum periods for $q=1.0, q=0.75$ and $q=0.5$. 
TABLE 5.3 .1

\begin{tabular}{lllclll}
$\frac{\mathrm{M}_{1}}{\mathrm{M}_{\odot}}$ & $\mathrm{R}_{1}$ & \multicolumn{3}{c}{$\mathrm{P}_{\mathrm{min}}$ (days) } & $\mathrm{P}_{\text {sync }}$ & (days) \\
4.40 & 24.7 & 20.6 & 20.1 & 19.1 & 70.6 & 57.6 \\
3.50 & 15.5 & 11.5 & 11.2 & 10.7 & 59.2 & 48.3 \\
2.80 & 10.0 & 6.7 & 6.5 & 6.2 & 43.7 & 35.7 \\
2.20 & 6.4 & 3.8 & 3.7 & 3.6 & 38.7 & 31.6 \\
1.75 & 4.7 & 2.7 & 2.6 & 2.5 & 48.7 & 39.8 \\
1.40 & 3.6 & 2.0 & 2.0 & 1.9 & 48.4 & 39.5 \\
1.25 & 3.2 & 1.8 & 1.8 & 1.7 & 50.6 & 41.3 \\
1.10 & 2.7 & 1.5 & 1.4 & 1.4 & 50.0 & 40.8 \\
0.90 & 2.2 & 1.2 & 1.2 & 1.1 & 52.4 & 42.8 \\
0.70 & 1.6 & 0.9 & 0.8 & 0.8 & 41.0 & 33.5
\end{tabular}

Table 5.3.1 Minimum periods for evolution without mass exchange, and maximum periods for tidal synchronisation for late Hertzsprung gap binary components of various masses.

The minimum orbital period increases rapidy with increasing primary mass. However, the dependence of $\mathrm{P}_{\mathrm{min}}$ on the mass ratio $q$ is so small as to be practically negligible over the range of $M_{1}$ and $q$ considered.

Having satisfied the requirement that the binary be detached, we now turn to the problem of determining the maximum orbital period for which a significant increase in the primary's axial rotation rate can be brought about due to tidal synchronisation during its lifetime as a Hertzsprung gap object with a convective envelope.

Zahn (1977) has investigated the problem of tidal friction in close binary systems, and derives an approximate 
expression for the synchronisation timescale of a binary component with a convective envelope

$$
\tau_{\text {sync }}(\text { years }) \simeq q^{-2}\left(\frac{a}{R_{1}}\right)^{6} \simeq q^{-2} \frac{M^{2} P^{4}}{R_{1}^{b}}
$$

where $M$ is the system mass in solar units, and $P$ is the orbital period in years.

If at least partial synchronisation is to be achieved in the primary, a likely upper limit on the orbital period is obtained by setting $\tau_{\text {sync }}=T_{\mathrm{CHG}}$, where $\mathrm{T}_{\mathrm{CHG}}$ is the star's convective Hertzsprung gap lifetime.

If $R_{1}$ is the radius of the primary at the base of the giant branch

$$
\mathrm{P}_{\text {sync }} \simeq\left(\frac{\mathrm{T}_{\mathrm{CHG}} \mathrm{q}^{2} \mathrm{R}_{1}^{6}}{\mathrm{M}_{1}^{2}(1+\mathrm{q})^{2}}\right)^{\frac{1}{4}}
$$

The values of $\mathrm{T}_{\mathrm{CHg}}$ for stars of various masses are listed in Table 5.7.1. The corresponding values of $P_{\text {sync }}$ are given for $q=1.0$ and $q=0.5$ in Table 5.3 .1 .

The dependence on $q$ is rather more significant here; synchronous axial rotation is more difficult to achieve in the primary if the secondary is of low mass. Once again, the critical period is strongly dependent upon the primaxy's radius at the base of the giant branch.

$$
\text { Comparison of the values of } \mathrm{P}_{\text {sync }} \text { given in Table 5.3.1 }
$$
with the observational data on the long-period RS CVn systems (Hall, 1976) shows broad agreement with the 
predicted behaviour of a sample of stars with $\mathrm{P} \simeq \mathrm{P}_{\text {sync }} \lambda$ And, $\alpha$ Aur and HK Lac all have orbital periods close to $\mathrm{P}$ sync' and photometric periods which indicate that varying degrees of synchronism have been achieved. In all the known RS CVn systems with $\mathrm{P}_{\text {orb }}<18$ days for which rotation periods have been determined from the photometric variations, the primary is rotating synchronously.

In an investigation of the incidence of spectroscopic binaries among main sequence and giant stars, Jaschek and Gomez (1970) found that, after correcting for selection effects, some $47 \%$ of all main sequence stars showed radial velocity variations indicative of duplicity, independent of spectral type.

Kraitcheva et.a1. (1978) have performed an extensive statistical analysis of the physical parameters of 826 spectroscopic binary systems. They find that the observed number of systems whose separations are such that they will enter the initial mass exchange phase while the more massive star is burning hydrogen in a shell (Case B) comprises some $70 \%$ of the total number of spectroscopic binaries. 25\% are shorter-period systems, which will undergo mass exchange during the more massive star's core hydrogen-burning lifetime (Case $A)$. The remaining 50 will enter the mass-transfer phase during the growth of a carbon-oxygen core in the more massive star (Case C).

These figures are seriously influenced by selection effects, of which the most important is the rather low number of stars in the third category which have been studied in detail to date. Kraitcheva et.al. calculate, for instance, that if stars of all masses have the same initial distribution of orbital semi-major axes $a$, and all 
values of $\log$ a in the range $1 \leqslant \log \left(\frac{a}{R_{\odot}}\right) \leqslant 4$ occur with equal probability, the short, medium and long period categories described above should comprise $20 \%, 40 \%$ and $40 \%$ of all spectroscopic binaries.

The upper limits $\mathrm{P}_{\max }$ on the orbital period for case $\mathrm{B}$ evolution are Iisted in Table 5.3.2., calculated for the primary radius at the tip of the giant branch using Eq. (5-5).

Although giant branch models have only been published for stars with masses of $2.2,1.75,1.4,1.1$ and $0.9 \mathrm{M}_{\odot}$ (Sweigart and Gross, 1978), radii at the highest point on the giant branch were estimated from a linear least-squares fit in the $(\log R, \log M)$ plane. Iben's (1967) model for a star of 5 solar masses was used to extend the relation to more massive stars.

The fraction $\beta$ of stars which will undergo Case $B$ evolution and achieve synchronous rotation before the end of the primary's Hertzsprung gap evolution is then given by

$$
\beta=\frac{\log P_{\text {sync }}-\log P_{\min }}{\log P_{\max }-\log P_{\min }}
$$

where the various limiting periods are given by Eqs. (5-5) and (5-7) and are tabulated as functions of $M_{1}$ and $q$ in Tables 5.3.1 and 5.3.2. The corresponding values of $\beta$ are also listed in Table 5.3.2.

It should be noted that the orbital separations corresponding to $\mathrm{P}_{\min }$ in Table 5.3.1 are less than $10 \mathrm{R}_{\odot}$ for the models with $\mathrm{M}_{1} \leqslant 1.4 \mathrm{M}_{\odot}$.

Kraitcheva et al (1978) have observed that there are no known detached binaries with $\mathrm{M}_{1}>1.5 \mathrm{M}_{\odot}$ and $\mathrm{a}<10 \mathrm{R}_{\odot}$. They have concluded that the critical orbital separation $a \simeq 10 \mathrm{R}_{\odot}$ probably corresponds to the maximum angular momentum which a protostar may accommodate without undergoing fission to form a binary system. 
The only known detached binaries with $\mathrm{a}<10 \mathrm{R}_{\odot}$ are active lower main sequence binaries and RS CVn systems with orbital periods less than about two days. Hall, Kreiner and Shore (1980) have found considerable evidence that the orbital periods of these systems are decreasing on mean timescales of $\sim 10^{7}$ years. If this is so, the presence of these systems in this "forbidden" range of orbital separations is an artifact of their mass and angular momentum loss. This, in turn, is attributable to the effects of fast axial rotation on the convective envelopes of stars with $M \leqslant 1.5 \mathrm{M}_{\odot}$. The evolutionary timescales for such systems are sufficiently long that the long-term period changes will be large enough to explain their present separations.

For the purposes of calculating $\beta$ using Eq. 5-8, $\mathrm{P}_{\min }$ was taken to be the period corresponding to an orbital separation $\mathrm{a}=10 \mathrm{R}_{\odot}$, for the systems with $\mathrm{M}_{1} \leqslant 1.4 \mathrm{M}_{\odot}$. Although evolved systems are likely to have shorter periods, we are interested only in the distribution of periods on the zeroage main sequence. In any case, the effect on $\beta$ is almost negligibly small. 
TABLE $5 \cdot 3 \cdot 2$

$\frac{M}{M_{\odot}} \quad \frac{R}{R_{\odot}} \quad P_{\max }($ days) $\quad q=1.0 \quad q=0.5$

$\begin{array}{llccll}4.40 & (80) & (120) & (110) & 0.69 & 0.62 \\ 3.50 & (90) & (170) & (150) & 0.61 & 0.57 \\ 2.80 & (100) & (220) & (200) & 0.54 & 0.50 \\ 2.20 & 114.7 & 292 & 271 & 0.53 & 0.50 \\ 1.75 & 147.6 & 478 & 443 & 0.57 & 0.53 \\ 1.40 & 156.7 & 585 & 542 & 0.58 & 0.51 \\ 1.25 & (160) & (630) & (580) & 0.55 & 0.51 \\ 1.10 & 166.6 & 723 & 670 & 0.56 & 0.52 \\ 0.90 & 174.8 & 859 & 796 & 0.54 & 0.50 \\ 0.70 & (210) & (1330) & (1230) & 0.52 & 0.51\end{array}$

Table 5.3.2 primary radius at tip of giant branch maximum orbital period for Case $B$ evolution and fraction $B$ of Case $B$ system primaries with tidallyenhanced axial rotation at base of giant branch, as functions of primary mass and mass ratio. parentheses denote radii obtained by interpolation.

Again, the dependence on $\mathrm{q}$ is rather weak. For primary masses less than about $5 \mathrm{M}_{\odot}$, the binaries which are like ly to show RS CVn characteristics when the primary is in the late stages of its Hertzsprung gap evolution form a subset comprising between $50 \%$ and $70 \%$ of the total number of Case B binaries.

Kraitcheva et.al. (1978) conclude that Case B binaries comprise some $40 \%$ of all spectroscopic binaries. Jaschek and Gomez (1970) find that some $47 \%$ of all main sequence stars are spectroscopic binaries, and it is reasonable to assume that a similar fraction of Hertzsprung gap stars also belong to close binary systems. 
If we combine the above figures, we find that about 10\% of all late Hertzsprung gap stars with any given mass $M \leqslant 5 M_{\odot}$ will be tidally-synchronised members of detached binary systems.

This provides a convenient definition for an RS CVn binary in evolutionary terms, as it requires that the orbital period and the spectral class of the active component lie within the ranges observed among the RS CVn binaries. It is also capable of reproducing the observed mass distribution observed among these binaries, as will be discussed in Section 5.7 .

\section{$5.4 \quad$ MAIN SEQUENCE SYSTEMS}

The most apparently numerous main sequence stars exhibiting RS CVn-like surface activity are the BY Draconis variables. The principal characteristics of these stars, adopted as a working definition by Bopp et. al. (1981) are

(a) low-amplitude photometric variability modulated with the rotation period;

(b) $d K$ or $d M$ spectral type; and

(c) Ca II (and in some cases hydrogen) emission features in the spectrum.

The rotation periods are of the order of ten days or less. The rapid rotation is due in some cases to tidal synchronisation in close binary systems; in others it is apparently a characteristic of stellar youth.

Unlike the late Hertzsprung gap stars, then, tidal interaction in a close binary is not necessary to induce the rotation rates required to produce the high levels of 
surface and chromospheric activity observed in the BY Dra stars.

The BY Dra syndrome has not been observed in main sequence $G$ stars, with the exception of a small number of binaries which Hall (1976) has classified as short-period RS CVn systems. These are characterised by orbital periods between about 0.5 and 1.0 days, strong $\mathrm{Ca}$ II $\mathrm{H}$ and $\mathrm{K}$ emission, photometric variations indicating starspot activity and synchronous rotation, and the active component being a $G$ dwarf. Some of the shorter-period double-lined RS CVn systems (notably TY PYx, AR Lac and HD 155555, with orbital periods $3.2,2.0$ and 1.7 days respectively) also exhibit strong $\mathrm{Ca}$ II emission from their G dwarf secondary components.

However, longer-period systems containing G dwarf secondaries (e.g. HD 101309, $\mathrm{P}=11.7 \mathrm{~d}$ and $\mathrm{LX}$ Per, $\mathrm{P}=8.0 \mathrm{~d}$ ) show little, if any, sign of enhanced activity in the secondary.

Recent studies by Vaughan et.al. (1981) of Ca II emission fluxes in lower main sequence stars have confirmed that a tight correlation exists between axial rotation period and $\mathrm{Ca}$ II emission strength measured relative to the surrounding photospheric spectrum, for single stars with spectral types later than about F5 V. The correlation has a strong temperature dependence built into it, due at least in part to the rapidly increasing photospheric flux at the wavelength of the $H$ and $K$ lines with increasing effective temperature: In order for the $\mathrm{H}$ and $\mathrm{K}$ emission to be detectable in an $\mathrm{F}$ or $\mathrm{G}$ dwarf, a very high absolute Ca II surface emission flux is required and hence, presumably, a correspondingly high rotation rate. 
The short-period RS CVn-like systems occupy a period range which is below the minimum limit for detached systems with subgiant components, but above the limit at which the components must evolve in contact, i.e. $0.3 \leqslant P$ (days) $\leqslant 1.3$ for a primary mass of $I \mathrm{M}_{\odot}$. The synchronisation timescale for a $q \simeq 1$ system with an orbital period of 1.3 days is of the order of $10^{5}$ years.

It seems that these systems have the good fortune to be the most active of the main sequence $G$ binaries, having the fastest axial rotation rates while occupying a range of orbital periods in which neither $W$ UMa nor RS CVn systems are found. Binaries with $G$ dwarf primaries and rather longer orbital periods are expected to show activity levels which decrease with increasing orbital period, and will evolve into RS CVn systems as their primaries cross the Hertzsprung gap. The limiting orbital period for a $q=1$ main sequence binary with $M_{1}=1 M_{\odot}$ and tidally-synchronised rotation of the primary is about 30 days, at which period ${ }^{\text {s }}$ sync $\approx T_{\mathrm{ms}}$. In any case, a $G$ dwarf with a rotation period of 30 days is unlikely to show much more extensive surface activity than that seen in the Sun, which has $P_{\text {rot }} \simeq 26$ days.

It thus appears that the short-period RS CVn systems form an extension of the BY Dra group to hotter effective temperatures.

\subsection{SEMI-DETACHED SYSTEMS}

The evolutionary fate of a binary with $M_{1} \approx 1 M_{\odot}$ and $0.5 \leqslant \mathrm{P} \leqslant 1.3$ days has been modelled by ziolkowski (1970), and is also discussed by Paczynski (1971). In this period range (Case A evolution) the primary overflows 
its Roche lobe while still burning hydrogen in a core. The resulting mass exchange more than reverses the mass ratio. Subsequent evolution proceeds on a slow, nuclear timescale as the primary (now less massive) star continues its core hydrogen burning while occupying the whole volume of its Roche lobe. This semi-detached subgiant is over-luminous for its mass by up to three magnitudes in some cases.

The evolutionary fate of the RS CVn binaries themselves will be such that the rapid mass transfer phase will begin while the primary is burning hydrogen in a shell (Case B evolution). Refsdal and Weigert (1969 a, b) and Giannone et.al. (1970) have modelled Case B evolution for various primary masses and mass ratios. In all these models, the end product of the mass exchange is a system in which the remnant of the primary has a degenerate helium core and continues to burn hydrogen in a shell on a timescale which is typically about $10 \%$ of the star's main sequence lifetime. This primary remnant fills its Roche lobe and may be over-luminous for its mass by up to ten magnitudes. The original secondary, however, is five to ten times as massive as the primary remnant and dominates the spectrum.

Several of the semi-detached RS CVn systems bear a close resemblance to such post-mass-transfer models, and as such should perhaps be classified as Algol-type binaries. HR 5110, AD Cap, RT Lac, RZ Cnc and AR Mon all have extreme mass ratios and over-luminous semi-detached subgiant components displaying $\mathrm{Ca}$ II $\mathrm{H}$ and $\mathrm{K}$ emission (Hall, 1976). The over-luminous, cool subgiant in Algol itself shows similar chromospheric emission features during primary eclipse. White et.al. (1980) report coronal X-ray 
emission from Algol similar to that associated with the RS CVn systems, and RS CVn-like radio emission has also been detected from this system (H jellming, Webster and Balick,1972). It thus appears that some form of dynamo mechanism must operate in these semi-detached objects, although their convective envelopes are of a very different nature to those in the evolved subgiant components in detached systems.

\section{$5.6 \quad$ GIANT SYSTEMS}

The RS CVn binaries, as discussed in section 5.3.3, form a short-period subset of binaries with primaries less massive than $5 \mathrm{M}_{\odot}$ evolving in Case $B$.

The remainder of the Case $B$ binaries will have orbital periods sufficiently long that their primaries will not have achieved synchronous rotation by the end of their Hertzsprung gap evolution. They will, however, expand to fill and eventually overflow their Roche lobes during their giant branch evolution.

By combining equations $(5-4)$ and $(5-6)$, it is readily seen that the snychronisation timescale varies roughly as $\left(R / R_{L}\right)^{-6}$. As the outer radius of the convective envelope approaches the Roche lobe radius, the synchronisation timescale decreases rapidly. For instance, a giant whose radius is only half that of its Roche lobe will have a synchronisation timescale of the order of $10^{4}-10^{5}$ years, according to Zalm's approximation. This is considerably less than the thermal timescale on which the expansion of the envelope takes place in stars with masses less than about $5 \mathrm{M}_{\odot}$. Consequently, by the time a giant in this mass range overflows its Roche lobe, it is highly likely that it will be co-rotating with its companion. 
Few such systems have been observed in detail to date. However, it appears that HR 4492 (Section 4.14) and several of the stars in Hall's (1976) list of long-period RS CVn systems fall into this category.

As in the RS CVn systems, the presence of a deep convective envelope and an enhanced axial rotation rate due to tidal friction leads to extensive surface activity. This manifests itself as enhanced Ca II (and in some cases hydrogen) emission, rotationally-modulated photometric variability, $\mathrm{x}$-ray and radio emission. Again, the conditions at the base of the convective envelope will differ considerably from those in the active RS CVn subgiants. However, the surface activity suggests that these conditions are capable of supporting hydromagnetic dynamos which operate on similar principles to those which produce the surface magnetic phenomena in late main sequence stars and in the RS CVn systems. 
5.7 OBSERVED AND THEORETICAL MASS AND LUMINOSITY FUNCTIONS FOR RS CVn SYSTEMS

The evolutionary definition of an RS CVn binary suggested in section 5.3 .3 may be tested by using it to predict a theoretical mass distribution and luminosity function for the RS CVn systems.

To do this, we must determine the initial mass function for the solar neighbourhood and the mass-luminosity function of late Hertzsprung gap objects.

The initial mass function, or IMF, is obtained by considering the solar neighbourhood as a cylinder of radius $\sim 1 \mathrm{kpc}$ with its axis passing through the sun perpendicular to the plane of the Galaxy. In order to avoid effects due to the observed concentration of high-mass stars close to the plane of the Galaxy, and the rather more extensive distribution of low-mass stars above and below the plane, all densities are integrated over the height of the cylinder (which extends several hundred parsecs above and below the Galactic plane) and expressed per square parsec.

The present day mass function (PDMF) of main sequence stars in the solar neighbourhood is defined as the number of main sequence stars per unit logarithmic mass interval per square parsec in the solar neighbourhood.

The PDMF has been determined by several investigators, notably McCuskey (1966), Luyton (1968) and Wielen (1974). It may be used to determine the relationship between the history of the stellar birthrate in the Galactic plane and the initial mass function (IMF) by assuming that the distribution of masses among newly-formed stars is independent of time. This distribution, called the IMF, is defined as the total number of stars ever formed per unit logarithmic mass 
per square parsec.

Miller and Scalo (1979) have investigated in detail the effects of various birthrate histories on the IMF, given the observed form of the PDMF for main sequence stars. They find that the average past birthrate may be at most 5 times larger or 3 times smaller than the present birthrate if a smoothly varying IMF is to be obtained. The IMF, the PDMF and the birthrate are related by

$$
\begin{aligned}
& \phi_{\mathrm{ms}}(\log M)=\frac{\xi(\log M)}{\mathrm{T}_{\mathrm{O}}} \int^{\mathrm{T}_{\mathrm{O}}} \mathrm{b}(t) d t, \mathrm{~T}_{\mathrm{ms}}<\mathrm{T}_{\mathrm{O}}(5-9 \mathrm{a}) \\
& \mathrm{T}_{\mathrm{o}}-\mathrm{T}_{\mathrm{ms}} \\
& \phi_{\mathrm{mS}}(\log M)=\frac{\xi(\log M)}{\mathrm{T}_{\mathrm{O}}} \int_{0}^{\mathrm{T}_{0}} \mathrm{~b}(t) d t, T_{\mathrm{ms}} \geqslant T_{0}(5-9 b) \\
& \text { where } \phi_{\mathrm{ms}}(\log M) \text { is the PDMF; } \xi(\log M) \\
& \text { is the IMF; } b(t) \text { is the birthrate as a } \\
& \text { function of time in units of the present } \\
& \text { birthrate; } T_{0} \text { is the age of the Galactic } \\
& \text { disc ; and } \mathrm{T}_{\mathrm{ms}} \text { is the main sequence } \\
& \text { Iifetime of a star of mass } M \text {. }
\end{aligned}
$$


Milier and scalo found that a constant birthrate

(i.e. $b(t) \equiv 1$ ) was most consistent with both theoretical birthrate history models and the observed IMFs for galactic clusters.

For the purposes of this investigation, the best-fit IMF derived by Miller and scalo for a constant birthrate is adopted, and an age $\mathrm{T}_{0}=12 \times 10^{9}$ years is assumed for the solar neighbourhood. The IMF takes the form of a Gaussian

$$
\xi(\log M)=C_{0} \exp \left[-C_{1}\left(\log M-C_{2}\right)^{2}\right]
$$

where the coefficients have values

$$
\begin{aligned}
& c_{0}=106.0 \\
& c_{1}=1.09 \\
& c_{2}=-1.02 .
\end{aligned}
$$

The uncertainties in the birthrate history do not affect $C_{1}$ and $C_{2}$ greatly, but render $C_{0}$ uncertain by a factor of 2. The uncertainty in $T_{0}$ introduces a further $10 \%$ uncertainty.

The present-day mass function of Hertzsprung gap stars may be obtained by replacing $\mathrm{T}_{\mathrm{ms}}$ in equations (5-9) with ${ }_{\text {CHG }}$ Equation $(5-9 b)$ gives the result that $\phi_{\text {CHG }}$ drops to zero for stars in which $\mathrm{T}_{\mathrm{ms}} \geqslant \mathrm{T}_{\mathrm{o}}$ ' which is to say that no star with $M \leqslant \sim 1 M_{\odot}$ can have evolved beyond the main sequence since the formation of the Galactic disk.

Equation 5-9(a) becomes

$$
\phi_{\mathrm{CHG}}(\log \mathrm{M})=\xi(\log \mathrm{M}) \frac{\mathrm{T}_{\mathrm{CHG}}}{\mathrm{T}_{\mathrm{O}}}, \mathrm{T}_{\mathrm{ms}}<\mathrm{T}_{\mathrm{O}}
$$

The values of $T_{\mathrm{CHG}^{\prime}} \xi(\log M)$ and $\phi_{\mathrm{CHG}}(\log M)$ are listed in Table 5.7.1 for stars with $I \mathrm{M}_{\odot}<M \leqslant 5 \mathrm{M}_{\odot}$, and $\phi_{\mathrm{CHG}}(\log \mathrm{M})$ is plotted in Fig. 5.7.1. 
TABLE $\quad 5.7 .1$

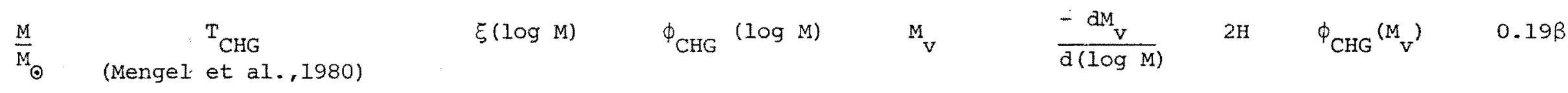

\begin{tabular}{|c|c|c|c|c|c|c|c|}
\hline 4.40 & $4.540 \times 10^{4}$ & 5.193 & $1.96 \times 10^{-5}$ & -0.82 & 9.5 & 180 & $1.15 \times 10^{-8}$ \\
\hline 3.50 & $2.325 \times 10^{5}$ & 7.367 & $1.43 \times 10^{-4}$ & 0.12 & 9.3 & 180 & $8.54 \times 10^{-8}$ \\
\hline 2.80 & $6.130 \times 10^{5}$ & 10.15 & $5.18 \times 10^{-4}$ & 1.01 & 8.8 & 185 & $3.18 \times 10^{-7}$ \\
\hline 2.20 & $3.387 \times 10^{6}$ & 14.02 & $3.96 \times 10^{-3}$ & 1.90 & 7.8 & 195 & $2.60 \times 10^{-6}$ \\
\hline 1.75 & $3.431 \times 10^{7}$ & 18.63 & $5.33 \times 10^{-2}$ & 2.60 & 6.3 & 230 & $3.68 \times 10^{-5}$ \\
\hline 1.40 & $1.064 \times 10^{8}$ & 24.08 & $2.14 \times 10^{-1}$ & 3.14 & 6.0 & 325 & $1.10 \times 10^{-4}$ \\
\hline 1.25 & $2.045 \times 10^{8}$ & 27.21 & $4.64 \times 10^{-1}$ & 3.42 & 6.0 & 415 & $1.86 \times 10^{-4}$ \\
\hline 1.10 & $4.191 \times 10^{8}$ & 31.05 & 1.08 & 3.76 & 6.0 & 510 & $3.54 \times 10^{-4}$ \\
\hline 0.90 & $1.158 \times 10^{9}$ & 37.67 & 0.0 & - & - & - & - \\
\hline 0.70 & $1.763 \times 10^{9}$ & 46.88 & 0.0 & - & - & - & - \\
\hline
\end{tabular}

Table 5.7.1 Hertzsprung gap lifetimes, initial mass function, present day Hertzsprung gap mass function and luminosity function, scale heights and proportion of RS CVn binaries for stars with $0.7 \leqslant M \leqslant 4.4$. 


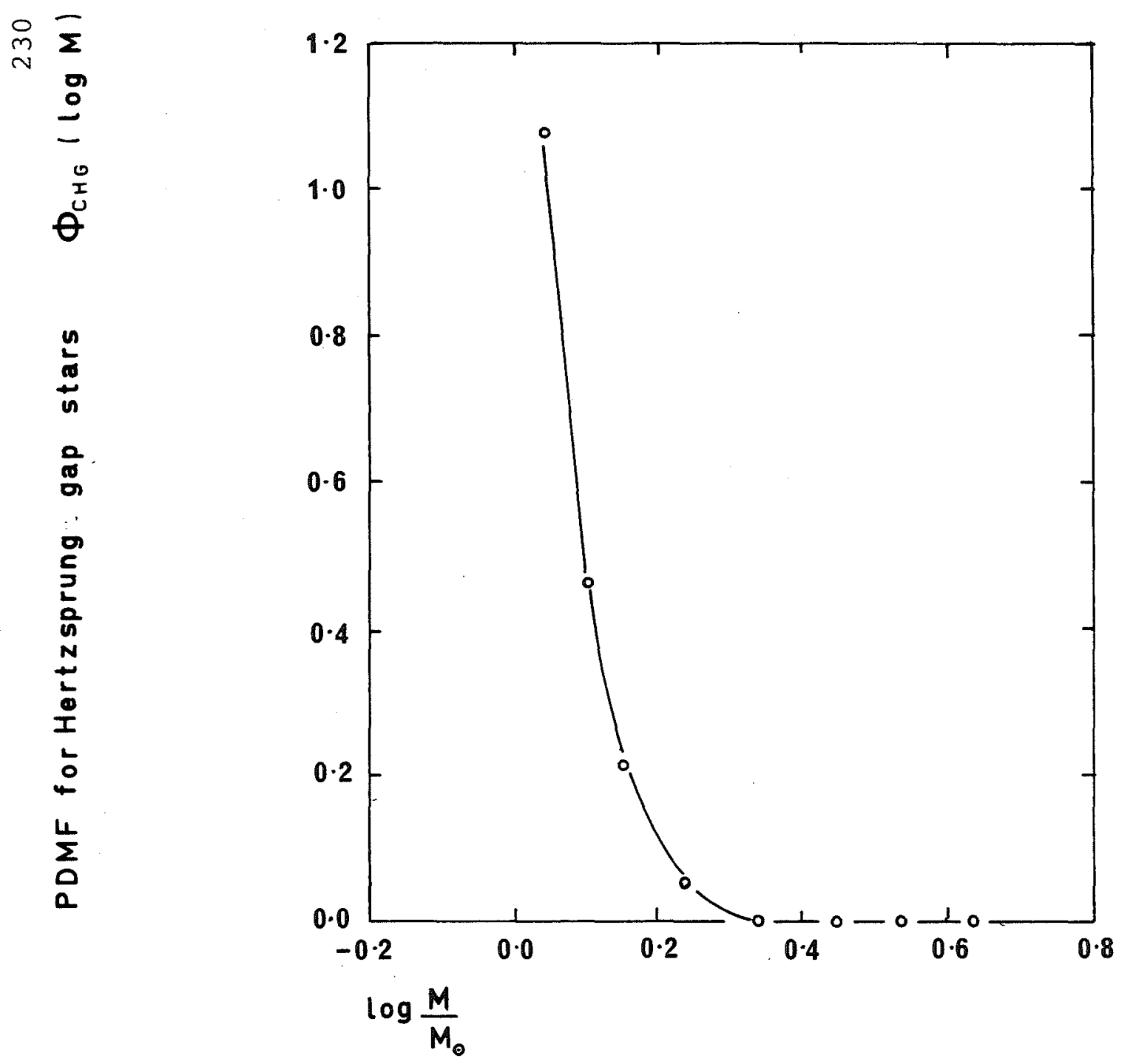

Fig. 5. 7.1 (a)
Theoretical mass function for Hertzsprung gap stars with $M<5 M_{\odot}$.

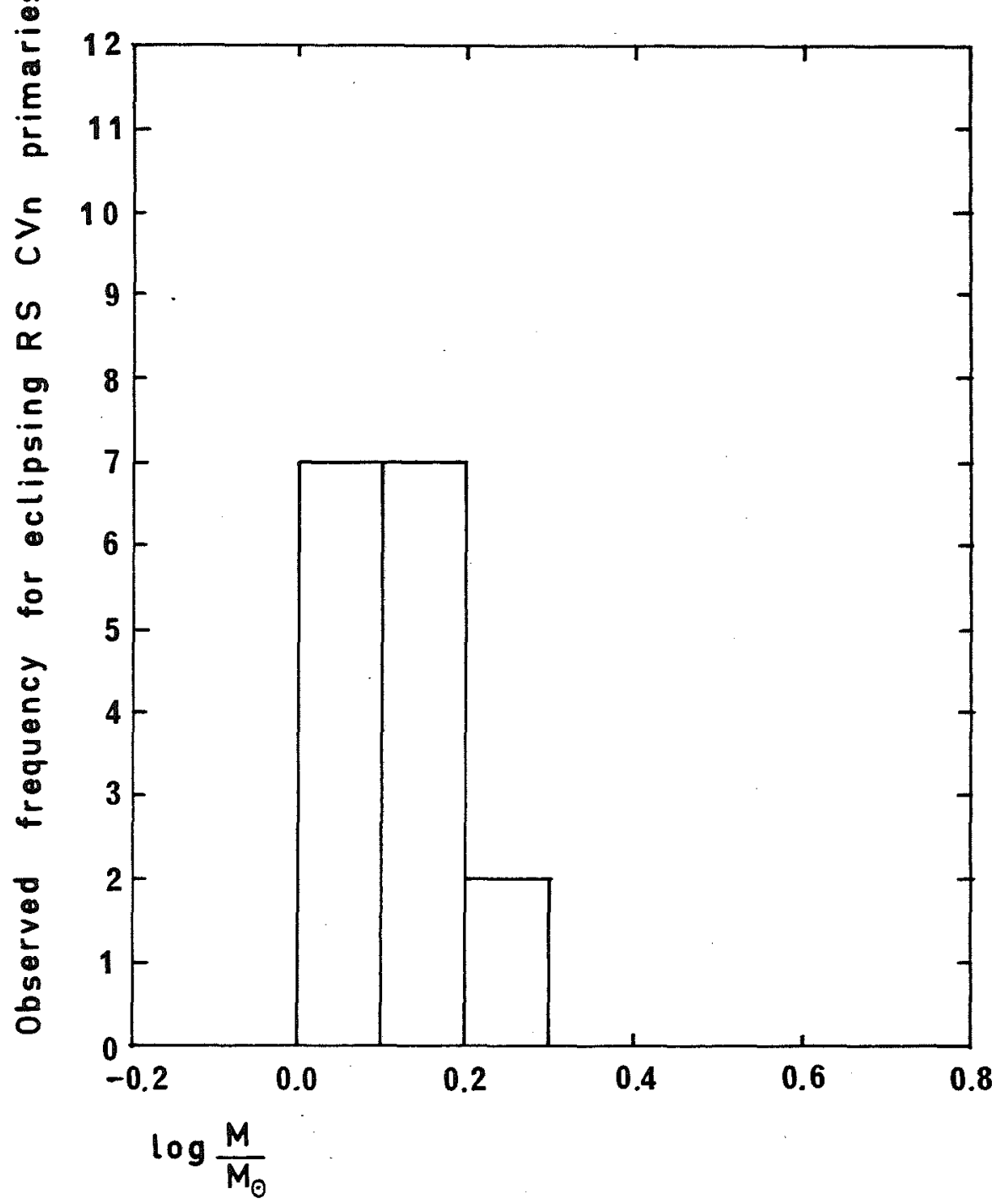

(b) Mass distribution of Hertzsprung gap primaries in eclipsing RS CVn systems (Popper and Ulrich,1977). 


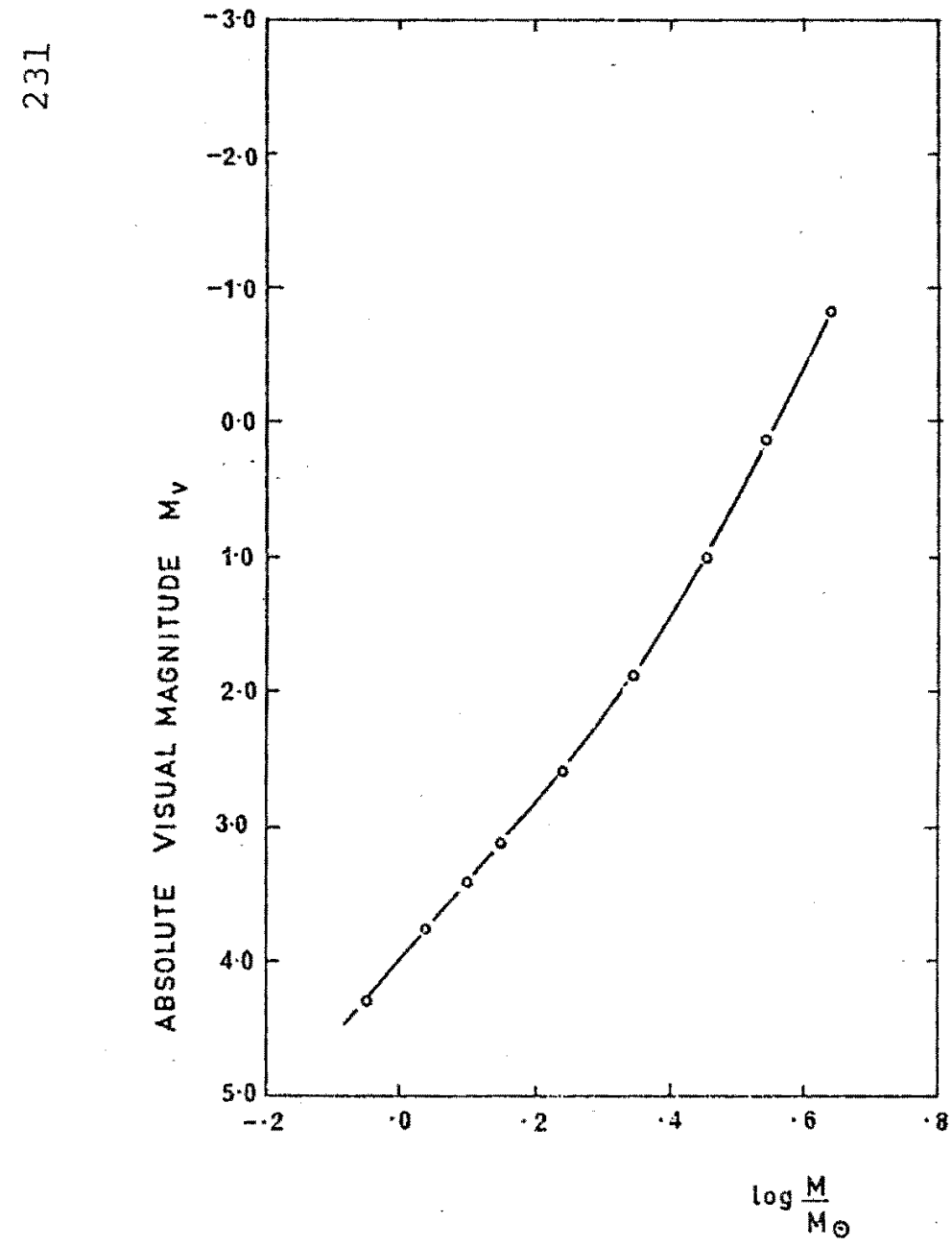

Fig. 5.7.2 Mass-luminosity relation for stars at base of giant branch with $M<5 M_{\odot}$.

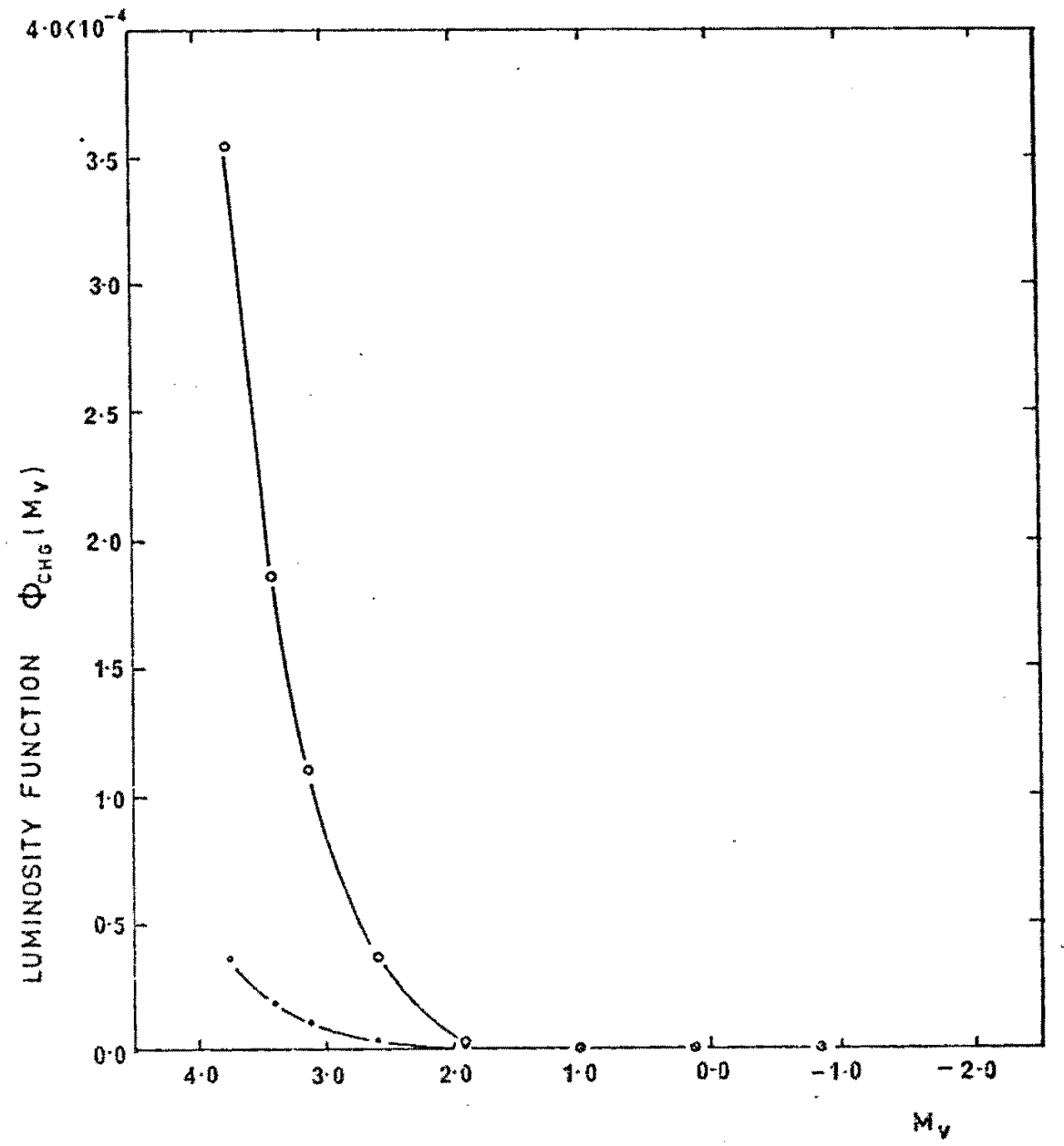

Fig. 5.7.3 Theoretical luminosity functions for late Hertzsprung gap stars (open circles) and RS CVn systems (dots), with $M<5 M_{0}$. 
The Iuminosity function $\phi_{\mathrm{CHG}}\left(\mathrm{M}_{\mathrm{v}}\right)$ is defined as the number of Hertzsprung gap stars per unit absolute magnitude and per cubic parsec, in the Galactic plane. The Iuminosity function is derived from the PDMF by

$$
\phi_{C H G}\left(M_{v}\right)=\frac{\phi_{C H G}(\log M)}{\left|\frac{d M v}{d(\log M)}\right| 2 H(\log M)}
$$

where $\mathrm{H}(\log \mathrm{M})$ is the scale height of the luminosity function for stars of given logarithmic mass. It is assumed that the luminosity function varies with perpendicular distance $z$ from the plane of the Galaxy according to

$$
\phi(z)=\phi(z=0) e^{-\left|\frac{z}{H}\right|} .
$$

The values of $2 \mathrm{H}$ given in Table 5.7.1 were obtained from the mean relation between $\log \frac{\mathrm{M}}{\mathrm{M}_{\odot}}$ and $2 \mathrm{H}$ adopted by Miller and Scalo (1979).

Fig. 5.7.2 gives the mass-1uminosity function for stars of various masses at the base of the giant branch taken from the models of Mengel et al. (1980). All the models have effective temperatures near $4800 \mathrm{~K}$, and a bolometric correction of -0.30 magnitudes (Johnson, 1966) was assumed in all cases.

The luminosity function for Hertzsprung gap stars obtained from Eq. (5-11) is given in Table 5.7.1 and in Fig. 5.7.3. The reasons for the observed range of primary masses among the RS CVn systems are at once apparent from Figs.5.7.1 and 5.7.3. For stars more massive than $\sim 2 \mathrm{M}_{\odot}$, the Hertzsprung gap lifetime is so short that at any time we expect to see 
very few such objects, compared with less luminous but more numerous and more long-lived Hertzsprung gap stars of between 1.0 and 1.6 solar masses.

Having determined the luminosity function for Hertzsprung gap stars, we may estimate the luminosity function for the RS CVn binaries, bearing in mind the constraints discussed in section 5.3.3. The values of $\phi_{\mathrm{CHG}}(\log M)$ and $\phi_{\mathrm{CHG}}\left(\mathrm{M}_{\mathrm{V}}\right)$ may be simply multiplied by the $47{ }^{\circ}$ duplicity factor of Jaschek and Gomez (1970), the $40 \%$ factor of Kraitcheva et.al. (1978) for binaries evolving in Case B, and the factor $\beta$ representing the fraction of Case $B$ binaries which may be considered as members of the RS CVn class, in each mass range.

The resulting fraction of Hertzsprung gap stars belonging to RS CVn binaries is listed in the last column of Table 5.7.1, and gives the luminosity function for the RS CVn binaries represented by the solid curve in Fig.5.7.3.

The theoretical space density of RS CVn systems is obtained by integrating the luminosity function over all absolute visual magnitudes $\mathrm{M}_{\mathrm{V}}$, i.e.

$$
\psi_{\mathrm{RS}}\left(\mathrm{pc}^{-3}\right)=0.19 \int_{-\infty}^{4.0} \beta_{\mathrm{V}}\left(\mathrm{M}_{\mathrm{V}}\right) \phi_{\mathrm{CHG}}\left(\mathrm{M}_{\mathrm{V}}\right) \mathrm{dM} \mathrm{V}
$$

Numerical evaluation of $(5-13)$ gives

$$
\psi_{\mathrm{RS}}=1.88 \times 10^{-5} \mathrm{pc}^{-3}
$$

The Iuminosity function for the RS CVn systems may also be used to derive the expected number of RS CVn systems brighter than a given limiting apparent visual magnitude,

$$
N\left(m_{v}<m\right)=\int_{-\infty}^{\infty} \phi_{R S}\left(M_{v}\right) \int_{0}^{2 \pi} \int_{-R\left(M_{v}, m\right)}^{R\left(M_{v}, m\right)} \exp \left[-\frac{|z|}{H\left(M_{v}\right)}\right] \int_{0}^{\left(R^{2}\left(M_{v}, m\right)-z^{2}\right)^{\frac{1}{2}}} r d r d \theta d M_{v}
$$




$$
\begin{aligned}
& \text { where } R\left(M_{v}, m\right)=10^{I+0.2\left(m-M_{v}\right)} \text { (parsecs), } \\
& \phi_{\mathrm{RS}}\left(\mathrm{M}_{\mathrm{V}}\right)=0.19 \beta\left(\mathrm{M}_{\mathrm{V}}\right) \phi_{\mathrm{CHG}}\left(\mathrm{M}_{\mathrm{V}}\right) \text {, } \\
& \text { and, } r, z, \theta \text { are cylindrical polar coordinates } \\
& \text { in which } \mathrm{z} \text { is perpendicular distance } \\
& \text { above the Galactic plane in parsecs; } \\
& r \text { and } \theta \text { are heliocentric polar coordinates } \\
& \text { measured in the plane of the Galaxy. } \\
& r \text { is also measured in parsecs. }
\end{aligned}
$$

Equation (5-13) reduces to an integral over absolute magnitude of the form

$N\left(m_{V}<m\right)=2 \pi \int_{-\infty}^{\infty} \phi_{R S}\left(M_{V}\right)\left[R^{2} H-2 H^{3}+2\left(R H^{2}+H^{3}\right) \exp \left(-\frac{R}{H}\right)\right] d M_{V}$

$$
\begin{aligned}
\text { where } & \mathrm{R} & =\mathrm{R}\left(\mathrm{M}_{\mathrm{v}}, \mathrm{m}\right) \\
\text { and } & \mathrm{H} & =\mathrm{H}\left(\mathrm{M}_{\mathrm{v}}\right)
\end{aligned}
$$

The value of the integrand in Eq. (5-14) is plotted against $\mathrm{M}_{\mathrm{V}}$ in Fig. 5.7.4, for limiting apparent visual magnitudes of 7.0 and 8.0 .

The greater luminosities of the more massive stars add to their apparent number density, because for a given limiting apparent magnitude they are visible over a far greater volume of space than the low-mass systems. In spite of this, they are still greatly outnumbered by the systems with primary masses between 1.0 and $2.0 \mathrm{M}_{\odot} \cdot$ For instance, Fig. 5.7.4 indicates that of a theoretical total of $\sim 48$ RS CVn primaries brighter than $V=8.0$, only $\sim 3$ will have masses greater than $2.2 \mathrm{M}_{\odot}$. None are likely to be more massive than $2.8 \mathrm{M}_{\odot}$. A comparison of Fig.5.7.4 and 5.7 .1 (b) shows excellent qualitative agreement between the observations and theory. 


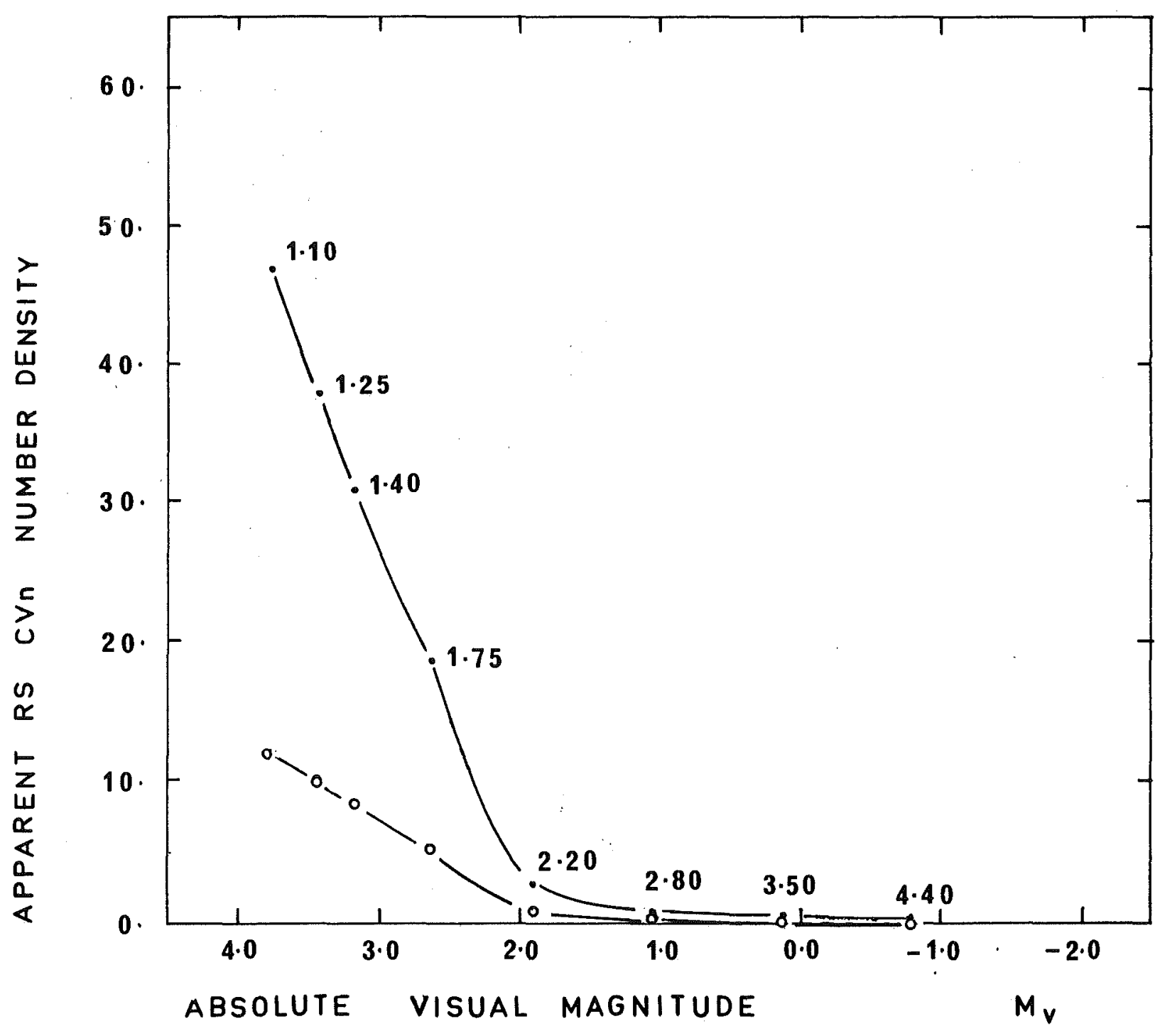

Fig. 5.7.4 Number density of synchronised, detached Hertzsprung gap binaries brighter than $V=8.0$ (dots) and $V=7.0$ (circles) as a function of absolute visual magnitude of the primary (more evolved) star. Points on the upper curve are labelled with their corresponding masses. 


\section{TABLE 5.7 .2}

$\begin{array}{ll}\text { Apparent } & \text { Predicted } \\ \text { Visual } & \text { Number } \\ \text { Magnitude } & \text { of RS CVn } \\ \text { Range } & \text { Systems }\end{array}$

Number of known

Detached RS CVn systems

This survey Popper \& Ulrich TOTAL

$9.0 \leqslant V<10.0 \quad 394$

0 (1977)

$8.0 \leqslant v<9.0 \quad 120$

6

$\geqslant 6$

$7.0 \leqslant V<8.0 \quad 35$

8

3

$\geqslant 8$

$6.0 \leqslant V<7.0 \quad 9$

3

$5.0 \leqslant \mathrm{v}<6.0$

3

0

1

$\mathrm{V}<5.0$

1

0

$\geqslant 6$

$\geqslant 1$

$\geqslant 0$

Table 5.7.2 Predicted and observed numbers of detached RS CVn systems for various apparent magnitude ranges. 
Table 5.7 .2 gives the expected numbers of RS CVn systems in various apparent magnitude ranges, for $1.0 \leqslant M_{\odot} \leqslant 4.4$. Considering the uncertainties built into the expression (5-10) used earlier for the initial mass function, the theoretical numbers bear a very close resemblance to the observed incidence of RS CVn systems in the brighter magnitude ranges.

Ha11 (1976) estimates the space density of the eclipsing RS CVn systems at between 1.0 and $2.0 \times 10^{-6} \mathrm{pc}^{-3}$. If we include non-eclipsing double-lined systems, this figure will increase by a factor of about 3 and inclusion of single-lined systems will give a further increase by a factor of at least 2 . This gives an adjusted space density between $6 \times 10^{-6}$ and $1.2 \times 10^{-5} \mathrm{pc}^{-3}$. Allowance for incompleteness in the very small sample of eclipsing binaries used for Hall's estimate brings the observed value even closer to the theoretical $1.9 \times 10^{-5} \mathrm{pc}^{-3}$.

It should be remembered that this theoretical space density in the Galactic plane is uncertain by an amount which depends on uncertainties in

(a) the empirical determinations of the PDMF for main sequence stars (Millex and Scalo, 1979);

(b) the frequency distribution for spectroscopic binaries of various masses and periods; and

(c) the convective Hertzsprung gap lifetimes of stars o varying composition, as compared with the values for $X=0.79 \quad Y=0.20$ and $z=0.01$ published by Mengel et al. (1980). The birthrate history and IMF both depend on the PDMF for main sequence stars. Fortunately, the various determinations of the PDMF for stars of about one solar mass and greater 
show good agreement; the uncertainty in $\log \phi_{\mathrm{ms}}\left(\mathrm{M}_{\mathrm{v}}\right)$ is about 0.15 for main sequence stars with $0.0 \leqslant \mathrm{M}_{\mathrm{v}} \leqslant 5.0$. This renders the space density uncertain by a factor of about 1.4. If we also consider the effects of the variation of evolutionary timescales for stars of different composition, and uncertainties in the results of Jaschek and Gomez (1970) and Kraitcheva et.al. (1978), the theoretical space density determined above is uncertain by a factor of about two.

The closeness of the observed RS CVn space density to the predicted space density of detached binaries with completely or partly synchronously rotating Hertzsprung gap primaries is remarkable, and provides a potentially useful solution to the much-discussed problem of defining an RS CVn binary in evolutionary terms. Moreover, this definition leads by the simple evolutionary arguments presented in this section to a theoretical mass distribution and an orbital period range which agree well with those observed among the most active $\mathrm{Ca}$ II $\mathrm{H}$ and $\mathrm{K}$ emission binaries. 


\section{ACKNOWLEDGMENTS}

During the course of this work, many people have provided me with invaluable assistance of one kind or another. of all these people, I particularly wish to thank my supervisor, John Hearnshaw, who kept a discreet but capable eye on my work and provided invaluable guidance in times of confusion. Mike Clark (Mt. John Observatory) displayed endless patience during many long hours spent on the telephone, diagnosing equipment problems at all hours of the night.

Don Mathewson (Director, Mt. Stromlo and Siding Spring observatories) made the spectroscopic work contained in this thesis possible, through the generous allocation of telescope and computer time during my stay in Australia. Alan Wright (CSIRO) was instrumental in turning the idea of the Parkes radio survey to reality. During that survey, John Hillier (Mt. Stromlo) spent four clear nights taking $\mathrm{H} \alpha$ spectra on the 74-inch telescope, only to be clouded out for his own run immediately afterwards.

Beverley Bristowe typed the original manuscript and my many subsequent alterations, and retained her sense of humour about it.

To these people, and to all the others who have helped, I extend my grateful thanks.

Finally, I wish to thank my parents, whose kindness and generosity have made my whole education possible. 
REFERENCES

Ayres, T.R., 1979. Astrophys. J. 228, 509.

Babcock, H.W., 1961. Astrophys. J. 133, 572.

Barnes, T.G. and Evans, D.S., 1976. Mon.Not.R.astr.Soc. 174, 489.

Barnes, T.G., Evans, D.S. and Parsons; S.B., 1976. Mon. Not. R. astr. Soc. 174, 503 .

Barnes, T.G., Evans, D.S. and Moffett, T.J., 1978. Mon. Not. R. astr. Soc. 183, 298 .

Belvedere, G., Chiuderi, C. and Paternò, L., 1982. Astron. Astrophys. 105, 133 .

Bennett, W.W., Evans, D.S. and Laing, J.D., 1962. Roy. Obs. Bull. No. 61 .

Bidelman, W.P. and MacConnell, D.J., 1973. Astron. J. 78,687. Biermann, P. and Hall, D.S., 1976. In IAU Symp.No.73, "Structure and Evolution of Close Binary Systems", eds. P. Eggleton et al. (Dordrecht:Reidel), p.381.

Bopp, B.W. (University of Toledo, Ohio, U.S.A.), 1981. Private Communication.

Bopp, B.W. and Noah, P.V., 1980. publ, astr. Soc. Pacific 92, 717 .

Bopp, B.W. and Stencel, R.E., 1981. Astrophys. J. 247, L131. Bopp, B.W. and Talcott, J.C., 1978. Astron. J. 83, 1517. Bopp, B.W. and Talcott, J.C., 1980. Astron. J. 85, 55. Bopp, B.W., Noah, P.V., Klimke, A. and Africano, J., 1981. Astrophys. J. 249, 210 .

Brault, J.W. and White, O.R., 1971. Astron. Astrophys. 13,169. Catalano, S. and Rodonò, M., 1967. Mem. Soc. astr. Ital. 38, 395 . 
Catalano, S. and Rodonò, M., 1969. In "Non-Periodic

Phenomena in Variable Stars", ed. L.Detre.

(Budapest:Academic Press), p.435.

Catalano, S. and Rodonò, M., 1974. Publ. astr. Soc.Pacific 86, 390 .

Chisari, D. and Lacona, G., 1965. Mem. astr. Soc. Ital. 36,463.

${ }^{*}$ Collier, A.C., 1982. Mon. Not. R. astr. Soc. 199, in press. Collier, A.C., Hearnshaw, J.B. and Austin, R.R.D., 1981.

Mon. Not. R. astr. Soc. 197, 769.

†Collier, A.C., Haynes, R.F., Slee, O.B, Wright, A.E. and Hillier, D.J., 1982. Mon. Not. R. astr. Soc. 200 , in press.

Cousins, A.W.J., 1973. Mem. R. astr. Soc. 77, 223.

Cousins, A.W.J., 1976. Mem. R. astr. Soc. 81, 25.

Durney, B.R., Mihalas, D. and Robinson, R.D., 1981. Astron. Astrophys. 93, 537 .

Durney, B.R. and Robinson, R.D., 1982. Astrophys. J., in press.

Eaton, J.A. and Hall, D.S., 1979. Astrophys. J. 227, 907.

Eggen, O.J., 1973. Publ. astr. Soc. Pacific 85, 42.

Eggen, O.J., 1978. Inf. Bull. var. Stars No. 1426.

Feldman, P.A. et al., 1978. Astron. J. 83, 1471.

Fraquelli, D.A., 1978. Astron. J. 83, 1535.

Garcia, M. et al., 1980. Astrophys. J. 240, L107.

Giannone, P., Refsdal, S. and Weigert, A., 1970. Astron. Astrophys . 4, 428 .

Gieseking, F., 1981. Astron. Astrophys. Suppl. Ser. 43,33 .

Griffin, R.F., 1972. Mon. Not. R. astr. Soc. 155, 449.

Grygar, J., Cooper, M.L. and Jurkevich, I., 1972. Bull. astr. Inst. Csl. 23,147 .

Hall, D.S., 1972.. Publ, astr. Soc. Pacific 84, 323. 
Ha11, D.S., 1976.

In IAU Coll. NO.29, "Multiply Periodic Phenomena in Variable stars", Part I, ed. W.S. Fitch (Dordrecht: Reidel),p.287.

Hall, D.S., Kreiner, J. and Shore, S.N., 1980. In IAU Symp. No. 88, "Close Binary Stars: Observations and Interpretation", eds. M.J. Plavec et al. (Dordrecht:Reidel), p. 383.

Hardie, R.H., 1962. In "Astronomical Techniques", ed. W.A. Hiltner (Chicago"Univ. of Chicago), p. 178 .

Hearnshaw, J.B., 1978. Astron. J. 83, 1531.

Hearnshaw, J.B., 1979. In IAU Coll. No. 46, "Changing Trends

in Variable Star Research", eds.

F.M. Bateson et al. (Hamilton, N.Z.:

Univ. of Waikatol, p.371.

Hearnshaw, J.B. and Oliver, J.P., 1977. Inf. Bull. var. Stars No. 1342 .

Hejlesen, P.M., 1980. Astron. Astrophys. Suppl. Ser. 39, 347. Hjellming, R.M., Webster, E. and Balick, B., 1972. Astrophys. J. 178, L139.

Houk, N., 1978. "Michigan Spectral Catalogue", Vol.II (Michigan: Univ, of Michigan).

Houk, N. and Cowley, A.P., 1975. "Michigan Spectral Catalogue", Vol. I (Michigan: Univ of Michigan).

Iben, I., Jnr., 1967. Ann. Rev. Astron. Astrophys. 5, 571. Jaschek, C. and Gomez, A.E., 1970, Publ. astr. Soc. Pacific 82,809 .

Johnson, H.L., 1966. Ann. Rev. Astron. Astrophys. 4, 193. Johnson, H.L., 1966a. In "Nebulae and Interstellar Matter", eds. B.M. Middlehurst and L.H. Aller (Chicago: Univ. of Chicago), p.167. 
Johnson, H.L., Mitchell, R.I., Iriarte, B. and Wisniewski,W.Z.,

1966. Comm. Lunar Planet. Lab. 4 , 99.

Joy, A.H., 1930. Astrophys. J. 72, 41.

Keller, G. and Limber, D.N., 1951. Astrophys. J. 113, 637." Kraitcheva, Z.T., Popova, E.I., Tutukov, A.V. and Yungelson,I.R., 1978. Astron. Zh. 55, 1176 (English Translation in Soviet Astronomy).

Kron, G.E., 1947. Publ. astr. Soc. Pacific 59, 261. Linsky, J.L., Worden, S.P., McClintock, W. and Robertson, R.M.

1979. Astrophys. J. Supp1. Ser. 41, 47.

Leighton, R.B., 1969. Astrophys. J. 156, 1.

Lucy, L.B. and Ricco, E., 1979. Astron. J. 84, 401.

Luyton, W.J., 1968. Mon. Not. R. astr. Soc. 139, 221.

McCuskey, S.W., 1966. In "Vistas in Astronomy", Vol. 7, ed.

A. Beer (Oxford:Pergamon), p.14l.

Maltby, P., 1972. Solar Phys. 26, 76.

Menge1, J.G., Sweigart, A.V., Demarque, P. and Gross, P.G.,1980.

Astrophys. J. Supp1. Ser. 40, 733.

Miller, G.E. and Scalo, J.M., 1979. Astrophys. J. Suppl.Ser.

41,513 .

Montle, R.E., 1973. Unpublished M.A. thesis, Vanderbilt

University, Nashville, Tennessee, U.S.A.

Necke1, H., 1974. Astron. Astrophys. 35, 99.

Paczynski, B., 1971. Ann. Rev. Astron. Astrophys. 9, 183.

Paku11, M.W., 1981. Astron. Astrophys. 104, 33.

Parker, E.N., 1955. Astrophys. J. 122, 293.

Parker, E.N., 1975. Astrophys. J. 198, 205.

Popper, D.M., 1961. Astrophys. J. 133, 148.

Popper, D.M., 1977. In "Highlights of Astronomy", Vo1. 4,

Part II., ed.E.A.Müller

(Dordrecht:Reidel), p.397. 
Popper, D.M. and Ulrich, R.K., 1977. Astrophys. J. 212, L131. Raveendran, A.V., Mekkaden, M.V. and Mohin, S., 1981. Inf. Bull. var. Stars No. 1975.

Refsdal, S. and Weigert, A., 1969: Astron. Astrophys. 6, 426. Reimers, D., 1973. Astron. Astrophys 24, 79.

Robinson, R.D. and Durney, B.R., 1982. Astron. Astrophys. (in press).

Rosner, R., Tucker, W. and Vaiana, G., 1978. Astrophys. J. $\underline{220}, 643$.

Rucinski, S.M., 1977. Publ. astr. Soc. Pacific 89, 280.

Rucinski, S.M., 1979. Acta Astr. 29, 203.

Rucinski, S.M., 1981 . Astron. Astrophys. 104, 260.

Sandage, A. and Eggen, O.J., 1969. Astrophys. J. 158, 685. Sanduleak, N. and Philip, A.G.D., 1968. Astron. J. 포 566. Shore, S.N. and Hall, D.S., 1980. In IAU Symp. No. 88,

$$
\begin{aligned}
& \text { "Close Binary Stars: Observations } \\
& \text { and Interpretation", eds. M.J. Plavec } \\
& \text { et al. (Dordrecht:Reidel), p. } 389 .
\end{aligned}
$$

Simkin, S.M., 1974. Astron. Astrophys. 31, 129.

Simon, T. and Linsky, J.L., 1980. Astrophys. J. 241, 759.

Sitterly, B.W., 1930. Contr.Princeton Univ. Obs. 11, 21.

Spangler, S.R., Owen, F.N. and Hulse, R.A., 1977. Astron. J.

$82,989$.

Stacy, J.G., Stencel, R.E, and Weiler, E.J., 1980. Astron. J. 85,858 .

Stapinski, T.E., Rodgers, A.W. and Ellis, M.J., 1979. Adv. Electronics and Electron Phys. 52, 389.

Stapinski, T.E., Rodgers, A.W. and Ellis, M.J., 1981. Publ. astr. Soc. Pacific $93,242$.

Steenbeck, M. and Krause, F. Astron. Nach. 291, 49. Stimets, R.W. and Giles, R.H., 1980. Astrophys. J. 242, L37. 
Strohmeier, W., Knigge, R. and Ott, H., 1965. Inf. Bull. var. Stars No. 100.

Sweigart, A.V. and Gross, P.G., 1978. Astrophys. J. Suppl.Ser. 36, 405 .

Thompson, K. (Monash University, Clayton, Victoria, Australia), 1982. Private Communication. Vaughan, A.H. et al., 1981. Astrophys. J. 250, 276. Vogt, s.S., 1981. Astrophys. J. 250, 327. Walter, F.M., and Bowyer, S., 1981. Astrophys. J. 245, 671. Walter, F.M., Linsky, J.L., Bowyer, S. and Garmire, G., 1980. Astrophys. J. 236, L137. Webbink,R.F., 1976. Astrophys. J. 209, 829. Weiler, E.J. et al., 1978. Astrophys. J. 225, 919. Weiler, E.J. and Stencel, R.E., 1980. Astron. J. 84, 1372. White, N.E., Holt, S.S., Becker, R.H., Boldt, E.A. and Serlemitsos, P.J., 1980. Astrophys. J. 239, L69.

Wielen, R., 1974. In "Highlights of Astronomy", Vol. 3, ed. G. Contopoulos (Dordrecht:Reidel), p. 395 .

Willstrop, R.V., 1964. Mem. R. astr. Soc. 69, 83. Wilson, O.C., 1968. Astrophys. J. 153, 221. Wilson, O.C., 1978. Astrophys. J. 226, 379. Wilson, O.C. and Bappu, M.K.V., 1957. Astrophys. J. 125, 661. Wood, D.B., 1972. "A Computer Program for Modeling Non-Spherical Eclipsing Binary star Systems" (Goddard space Flight Center, Greenbelt, Maryland, U.S.A. Document $x-110-72-473)$.

Wood, D.B., 1978. WINK Status Reports Nos. I to 9 (1973 - 1978), Private Communication. 
Worley, C.E., 1978. Publ. U.S. Naval Obs. Vol. XXIV, Part VI, p.89.

Worley, C.E., (U.S. Naval Observatory, Washington, D.C.), 1981. Private Communication.

Yoshimura, H., 1975. Astrophys. J. Supp1. Ser. 29, 467.

Zahn, J-P., 1977. Astron. Astrophys. 57, 383.

Ziolkowski, J., 1970. Acta. Astr. 20, 213. 
The work presented in this paper is based entirely on my own research.

\section{A.C. Collies.}

A. C. Collier. 
DISCOVERY OF TWO FK COMAE STARS IN THE SOUTHERN HEMISPHERE

$$
\text { A, C, COLLIER }
$$

DEPARTMENT OF PHYSICS

UNIVERSITY OF CANTERBURY

CHRISTCHURCH, 1,

NEW ZEALAND, 


\section{ABSTRACT}

Radial velocity measurements, high resolution $\mathrm{H} \alpha$ spectra and broad-band photometry are presented for two Jate $G$-early $\mathrm{K}$ southern giant stars, HD 32918 and HD 36705. Both stars are known Ca II emitters; the high rotational broadening found in their spectra $\left(V_{\mathrm{e}} \sin i \geqslant 50 \mathrm{kms}^{-1}\right)$ and lack of evidence for any radial velocity variations indicates that they are members of the FK Comae class of fast-rotating surface-active late-type giants. Photometry of HD 32918 shows an RS CVn-like distortion wave with an amplitude of 0.3 magnitudes in $V$, and a period of 9.55 days. Variable $\mathrm{H} \alpha$ emission is present in both stars. The space density of FK Comae stars is discussed; the results are broadly consistent with the hypothesis that the FK Comae stars represent a stage in the post-nainsequence evolution of $W$ Ursae Majoris systems. 


\section{INTRODUCTION}

A working definition of the FK Comae stars has been outlined by Bopp and stencel (1981). The three original members of the class are FK Com, UZ Lib and HD 199178. All three are G-K giants exhibiting strong chromospheric and transition region emission spectra at the $\mathrm{Ca}$ II $\mathrm{H}$ \& $\mathrm{K}$ lines and in the ultraviolet. Erratically variable emission is also observed at $H \alpha$. Broad band photometric variability similar to the "distortion wave" phenomenon in the RS CVn binaries is seen. High rotational broadening is present in both the absorption spectra and the Ca II emission reversals ; typical $\mathrm{v}_{\mathrm{e}} \sin i$ values are around $100 \mathrm{kms}^{-1}$. No radial velocity variations suggesting duplicity are present in any of these stars.

The evolutionary origins of such objects are of particular interest. Bopp \& stencel have interpreted their behaviour in terms of the post main sequence evolution of $W$ Ursae Majoris systems. A theoretical study of W UMa evolution by webbink (1.976) showed that coalescence of contact binary systems may occur following core hydrogen exhaustion in the primary, to produce a single fast rotating luminosity class III object.

The observations presented here were made during an investigation into the surface activity levels of a number of late-type stars in the southern hemisphere classified by Bidelman and Macconnell (1973) as exhibiting strong $\mathrm{Ca} I I \mathrm{H} \& \mathrm{~K}$ emission on objective prism plates. The program consisted of broad band photometric monitoring for variability, a radial velocity study seeking evidence of duplicity, and a high-resolution $H \alpha$ survey of the candidate stars. 
Two of the stars examined,HD 32918 and HD 36705, were found to behave in a similar manner to the known FK Comae stars. It is proposed that they be added to the list, bringing the total number of such objects known to five.

II. OBSERVATIONS

1. Photometry :

Broad-band differential photometry in the BVRI system of Cousins (1976) was carried out during 1979 and 1980 at Mount John University Observatory, using the $61 \mathrm{~cm}$ Bollex and Chivens telescope equipped with an RCA C $31.034 \mathrm{~A}$ photomultiplier cooled to $-30^{\circ} \mathrm{C}$. Three integrations of ten seconds each in photon counting mode per filter were made for each observation. All observations were corrected for extinction, using coefficients determined each night when possible or else mean values for each run. The results were then transformed to the cousins system.

The comparison and check stars used for HD 32918 were HD 33763 and HD 33747 respectively; neither was found to vary. Regular monitoring of HD 32918 during the 1979 August to 1980 February observing season revealed a quasi-sinusoidal variation in $V$, with a period of $9.55 \pm 0.02$ days and a total amplitude of $\sim 0.3$ magnitude (Fig. 1). The variation is accompanied by a colour change (Table I) suggestive of a longitudinal variation in surface temperature, similar to that which is believed to give rise to the "distortion wave" seen in the Iightcurves of RS CVn binaries (Eaton and Hall, 1979). The large amplitude of the wave implies a high degree of surface activity and an axial inclination such that the syste $\mathrm{n}$ is viewed nearly equator-on.

HD 36705 was observed on only one night (1979 August 10 U.T.), permitting a preliminary determination of colour indices (Table I). Variability zould not, however, be established, and further observations should be made. 
The broad band colours suggest spectral types of $\mathrm{Kl}-2$ III and G8 III for HD 32918 and HD 36705 respectively.

\section{Radial Velocity study :}

The radial velocity observations were made during 1980 October and November at Mount stromlo Observatory. Spectra were obtained in the region of the Mg I b lines near $5160 \AA$ in the second order of the " $C$ " grating (600 Jines $\mathrm{mm}^{-1}$, blazed at $12,000 \AA$ in the first order) at the coude focus of the $1.88 \mathrm{~m}$ telescope. The detector was the 1024-element red-sensitive photon counting array (PCA) described by stapinski, Rodgers and Ellis (1979). The effective dispersion of this configuration was $14 \AA$ $\mathrm{mm}^{-1}$, or about $0.17 \AA$ per reticon array element. A typical slit width of one arc second gave a resolution of about $0.4 \AA$.

The spectra were reduced using the interactive SPECT pack age on the $\operatorname{VAX} 11 / 780$ computer at Mt.stromlo. The radial velocities were determined using the cross-correlation method of Simkin (1974); the template spectrum used was that of HR 141 (spectral type KO III ; $v_{x}=18.7 \mathrm{kms}^{-1}$ ). Each spect rum was cross-correlated with the template, and the shift of the cross-correlation function (ccf) peak from the origin measured. Heliocentric corrections were then applied in order to obtain a heliocentric radial velocity for each spectrum. Five additional radial velocity standard stars were included a nong the program stars. These were HR 387, HR 406, HR 507, HR 8897 and HR 8928. The mean deviation of the measured radial velocities of 
these stars from their catalogued values (Griffin, 1972) was found to be $1.2 \mathrm{kms}^{-1}$.

Typical ccfs for HD 32918, HD 36705 and the radial velocity standard HR 406 are illustrated in Figure 2 . The degree of rotational smearing of the ccf peaks for HD 32918 and HD 36705 is typical of $v_{e}$ sin $i$ values of $50 \pm 10 \mathrm{kms}^{-1}$ and $70 \pm 10 \mathrm{kms}^{-1}$ respectively. The radial velocities as determined from the ccf shifts are summarised in Table II. The HD 32918 data give a mean radial velocity of $\bar{v}_{x}=-11.7 \mathrm{kms}^{-1}$, with standard error $s=2.0 \mathrm{kms}^{-1}$. The mean and standard error for HD 36705 are $\bar{v}_{r}=+28.0 \mathrm{kms}^{-1}$ and $\mathrm{s}=4.6 \mathrm{kms}^{-1}$. In the latter case, the increased standard error axises from the high rotational smealing of the ccf, making the determination of the shift rather uncertain. The variations in Table II appear to be random; no periodic variation appears in the data for either star.

\section{Ha spectra :}

A program of high resolution $H \alpha$ spectroscopy was carried out during 1980 December and 1981 February. During this survey, several spectra of both HD 32918 and HD 36705 were obtained using the êchelle spectrograph at the conde focus of the $1.88 \mathrm{~m}$ telescope at Mt. Stromlo. The detector was the same red PCA used for the radial velocity study. The effective dispersion was $2.8 \AA \mathrm{mm}^{-1}$ at the reticon, or $0.034 \AA$ per pixel; the resolution with a one arc second slit was about $0.2 \AA$. Reduction of the spectra consisted of a flat-field 
correction for removal of coherent noise, and application of a mild Gaussian filter prior to continuum fitting. Both HD, 32918 and HD 36705 exhibit variable emission at $H \alpha$. In the case of HD 32918, the profile is strongly reminiscent of that seen in the RS CVn binary HR 1099, with an emission feature whose FWHM is about $200 \mathrm{kms}^{-1}$. The feature varies somewhat in strength, and sometimes appears asymmetric. (Fig. 3(a)).

HD 36705 occasionally shows a very shallow absorption feature at $\mathrm{H} \alpha$, but more usually this is filled to continuum level (Fig. 3(b)). The H $\alpha$ profile of HD 157457 (spectral type G8 III) is included for comparison in Fig. 3 (c).

\section{DISCUSSION}

The present observations indicate that both HD 32918 and HD 36705 show evidence of. high surface activity levels in conjunction with extremely high rotational broadening of their spectra. The values of $v_{e}$ sin. $i$ observed for both these stars are rather higher than those typically observed in RS CVn systems; the lack of any firm evidence for radial velocity variations in their spectra indicates that they are single stars, rather than close binaries. As such, they appear to be similar to the FK Comae stars, and thus may be considered as new members of the class.

Lower limits on the radii of the FK Comae stars may be estimated from their $v_{e}$ sin $i$ ralues and their photometricdetermined rotation periods (Table III). In spite of the rather small sample available, it is probably valid to take 
the mean $R$ sin $i$ value thus obtained and correct it for the effect of random orientation of rotation axes, to get an order-of-magnitude estimate of the "typical" radius of an $\mathrm{FK}$ Com star.

The result is $R / R_{\odot} \sim 10$. Combined with the observed temperature range of the FK Com stars, this gives a probable range of absolute visual magnitudes $M_{v}=1.0 \pm 1.0$. This location on the HertzsprungRussell diagram corresponds more or less to the Hertzsprung gap for stars with masses in the range $1.5 \leqslant M_{\odot} \leqslant 3.0$ (Iben, 1967).

At the same time, the range of absolute magnitudes estimated above would imply distances no greater than $\sim 400$ pc for the five known FK Com stars. This in turn gives a lower limit on the space density of FK Com systems of about $2 \times 10^{-8} \mathrm{pc}^{-3}$.

At this stage, it is worth checking the consistency of these estimates with the coalesced W. Ursae Majoris system hypothesis proposed by Bopp and stencel for the evolutionary origins of the FK Com stars. For this purpose, the assumption will be made that, following the disruption of the secondary component in a $W$ UMa system evolving according to Webbink's (1976) scheme, the resulting single fast-rotating Hertzsprung gap object will attain a thermal equilibrium configuration similar in appearance and subsequent evolutionary behaviour to a single, nonrotating star of the same mass and composition.

The lifetime of a $1.5 \leqslant M / M_{\odot} \leqslant 3.0$ star in the Hertasprung gap stage of its evolution thus corresponds 
to the time in which we might reasonably expect to see the FK Com syndrome; envelope expansion and enhanced mass and angular momentum loss during the ascent of the giant branch will subsequently contribute to a rapid lowering of the equatorial rotation.

If, as we have assumed, the Iuminosities of the FK Com stars are reliable indicators of their masses, then their evolutionary progenitors would be $W$ UMa systems with total masses greater than about $1.5 \mathrm{M}_{\odot}$.

The space density of such high-mass $W$ UMa systems is about $3 \times 10^{-7} \mathrm{pc}^{-3}$ (Kraft, $1967 ;$ Van't Veer, 1975). The space density ratio

$$
\frac{\text { FK Com stars }}{\text { massive } W \text { UMa systems }} \geqslant 7 \times 10^{-2}
$$

thus suggests that the lifetirie of an FK Com star formed by the coalescence of a contact binary system would be roughly an order of magnitude less than its main sequence W UMa Iifetime.

Such a main sequence lifatime would thus be of the same order of magnitude as that for a single star of similar mass to the primary component in the contact system. It should, however, be noted that we are working with a lower limit on the FK Com space density. Nonetheless, no serious inconsistencies are found between the observed space density and that which might reasonably be estimated from Webbink's models using t ie assumptions made here. 
Pakull (1981) reports that HD 36705 has been detected at $\mathrm{X}$-ray wavelengths, and that a photometric wave with a minimum at JD $2444296.575 \pm 0.003$ and $a$ period of $0.51423 \pm 0.00005$ has been established from observations made between 1980 February and October. The period is by far the shortest observed to date among the FK Com stars. A trial mass of $2 \mathrm{M}_{\odot}$ for the star gives a maximum radius of $\sim 3.4 \mathrm{R}_{\odot}$, beyond which equatorial disruption would occur. The corresponding equatorial rotation velocity would be $\sim 360 \mathrm{~km} \mathrm{~s}^{-1}$ at breakup.

Examination of the phasing of the radial velocity data shows that fairly uniform coverage was obtained between phases 0.538 and 1.160. This confirms that no systematic radial velocity variation with rotational phase exists.

Examination of eleven.low-dispersion blue spectrograms of HD 36705 taken by Hearnshaw (1979) at Mount John indicated a luminosity class near IV from the line ratio $\lambda 4077 \mathrm{SrII} / \lambda 4071 \mathrm{FeI}$. This is probably consistent with a star of the above dimensions or smaller, rotating near equatorial breakup velocity.

The observed value of $v$ sin $i$ thus implies a low value for the axial inclination; the actual equatorial rotation speed is probably between $200 \mathrm{~km} \mathrm{~s}^{-1}$ and $300 \mathrm{~km} \mathrm{~s}^{-1}$, implying that the inclination: ies in the range $i \simeq 16^{\circ} \pm 4^{\circ}$. 
Again, the data are consistent with the Webbink model; in fact the observations bear a striking resemblance to the stage in the model's evolution immediately following coalescence. If $W$ UMa systems are the evolutionary ancestors of FK Com stars, HD 36705 is a likely candidate for the youngest FK Com star identified to date.

An interesting final note is that if, as the Webbink models suggest, the main sequence lifetime of a W UMa system is comparable with that of a single star with the same mass as the primary, we would not expect to see FK Com-like descendants of contact systems with total masses less than about $1.5 \mathrm{M}_{\odot}$. For less massive systems, the time required before core hydrogen exhaustion occurred in the primary would exceed the age of the Galaxy.

\section{ACKNOWLEDGMENTS}

I should like to thank the Director, staff and students of Mt.stromlo Observatory for their willing and able assistance and for the generous allocation of telescope and computer time during the course of this work. Financial assistance from the University Grants Committee of New Zealand is acknowledged.

I am also grateful to Dr. B.W. Bopp for many useful. discussions on the subject, and for drawing to my attention the work of Dr. M.W. Pakull on HD 36705. 
REFERENCES

BIDELMAN, W.P. and MacCONNELL, D.J., 1973. Astron. J. 78, 687.

BOPP, B.W. and STENCEL, R.E., 1981. Astrophys.J. (Lett) 247,L131. Cousins, A.W.J., 1976. Mem. R. Astr. Soc. 81, 25. EATON, J.A. and HALL, D.S., 1979. Astrophys. J.227, 907. GRIFFIN, R.F., 1972. Mon. Not. R. Astr. Soc. 155, 449 HEARNSHAW, J.B., 1979. IAU Colloquium No. 46, Changing Trends in Variable star Research (University of Waikato, Hamilton), 371.

IBEN, I. Jr., 1967. Ann. Rev. Astron. Astrophys. 5, 571. KRAFT, R.P., 1967. Pub. Astr. Soc. Pac. 79, 395. PAKULL, M.W., 1981. Astron. Astrophys, Letters (preprint). SIMKIN, S.M., 1974. Astron. Astrophys. 31, 129. STAPINSKI, T.E., RODGERS, A.W. and ELLIS, M.J., 1979. Advances in Electronics and Electron Physics $\underline{52}$, 389.

VAN'T VEER, F., 1975. Astron. Astrophys. 40, 167. WEBBINK, R.F., 1976. Astrophys. J. 209, 829. 
TABLE I

Magnitudes and Colour Indices for Program Stars

$\begin{array}{llcccc}\text { OBJECT } & & \mathrm{V} & (\mathrm{B}-\mathrm{V}) & (\mathrm{V}-\mathrm{R})_{\mathrm{C}} & (\mathrm{V}-\mathrm{I})_{\mathrm{C}} \\ \mathrm{HD} 32918 & (\mathrm{max}) & 8.60 & 1.04 & 0.61 & 1.16 \\ \mathrm{~N} & (\mathrm{~min}) & 8.89 & 1.12 & 0.65 & 1.23 \\ \text { HD } 36705 & & 6.94 & 0.86 & 0.49 & 0.93\end{array}$

TABLE II

Radial velocities of HD 32918 and HD 36705,1981 OctoberNovember

$\begin{array}{cccc}\text { HJD } & \mathrm{V}_{\mathrm{R}}(\mathrm{HD} 32918) & \mathrm{HJD} & \mathrm{V}_{\mathrm{R}}(\mathrm{HD} 36705) \\ 2444000+ & \left(\mathrm{km} \mathrm{s}^{-1}\right) & 2444000+ & \left(\mathrm{km} \mathrm{s}^{-1}\right) \\ 538.119 & -11.8 & 538.133 & +22.3 \\ 539.062 & -8.8 & 539.077 & +33.5 \\ 539.227 & -11.3 & 539.136 & +26.5 \\ & & 539.203 & +37.4 \\ 566.007 & -9.6 & 565.993 & +29.3 \\ 567.013 & -13.9 & 566.035 & +24.7 \\ 567.052 & -14.5 & 567.001 & +24.4 \\ 567.099 & -12.7 & 567.041 & +27.5 \\ 567.153 & -10.9 & 567.086 & +24.9 \\ & & 567.142 & +29.9\end{array}$




\section{TABLE III}

Some observational properties of the FK Comae stars (data on FK Com, UZ Lib and HD 199178 from Bopp and Stencel (1981)).

\begin{tabular}{|c|c|c|c|c|c|c|}
\hline Star & V & $\begin{array}{l}\text { Spectral } \\
\text { type }\end{array}$ & $\begin{array}{l}v_{e} \sin i \\
\left(k m s^{-1}\right)\end{array}$ & $\begin{array}{l}\text { Photometric } \\
\text { period } \\
\text { (days) }\end{array}$ & $\begin{array}{r}\text { velocity } \\
\text { variation } \\
\left(\mathrm{km} \mathrm{s}^{-1}\right)\end{array}$ & $\frac{R}{R_{0}} \sin i$ \\
\hline FK Com & 8.2 & G2 II Ia & 100 & 2.40 & $\leqslant \pm 20$ & 4.7 \\
\hline HD 199178 & 7.3 & G5 III/IV & 90 & $\sim 4:$ & $\leqslant \pm 3$ & 6.3 \\
\hline UZ Lib & 9.2 & KO III & 80 & 4.75 & $\leqslant \pm 25$ & 8.4 \\
\hline HD 32918 & 8.6 & K2 III* & 50 & 9.55 & $\leqslant \pm 3$ & 9.4 \\
\hline HD 36705 & 6.9 & G8 III* & 70 & $0.514^{\dagger}$ & $\leqslant \pm 5$ & 2.7 \\
\hline
\end{tabular}


FIGURE CAPTIONS

Fig. I : Lightcurve of HD 32918, plotted against rotational phase determined using the ephemeris HJD $=2444155.64+9.5476 \mathrm{E}$. Crosses represent 1979 August data; dots, 1979 October ; Circles, 1980 January ; squares, 1980 February.

Fig. 2 : Cross-correlation functions of Mg I bline region spectra in (a) HD 32918 ; (b) HD 36705 ; (c) HR 406, giving radial velocity shifts with respect to template star HR 141. No heliocentric corrections have been made ; for corrected velocities, see text. Note rotational broadening of c.c.f. peaks in HD 32918 and HD 36705 , compared with HR 406 .

Fig. 3 : Typical Ha profiles in (a) HD 32918, (b) HD 36705 , (c) HD 157457. Sharp absorption features in (a) and (b) are telluric $\mathrm{H}_{2} \mathrm{O}$ lines. 


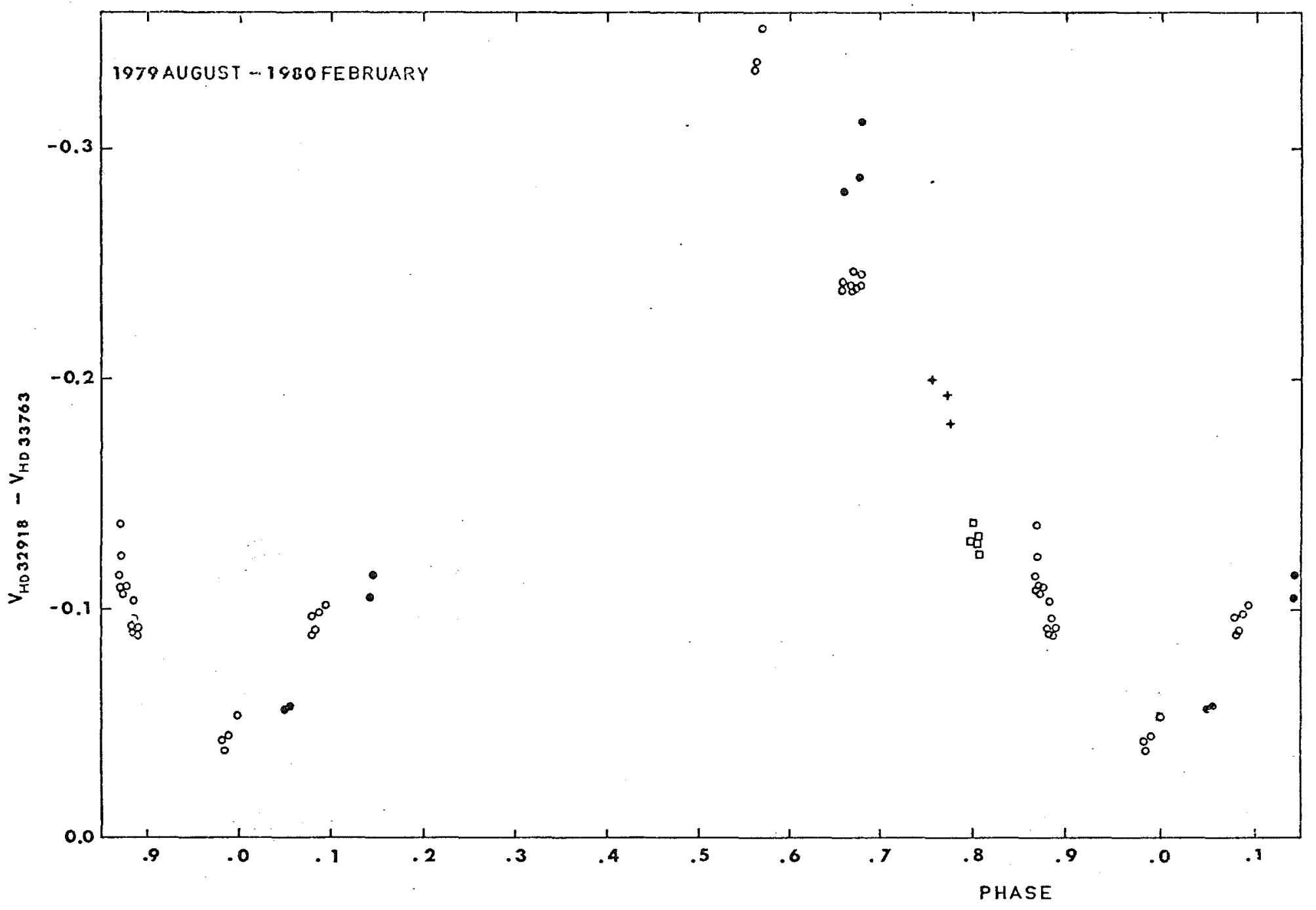


Figure 2. (a)

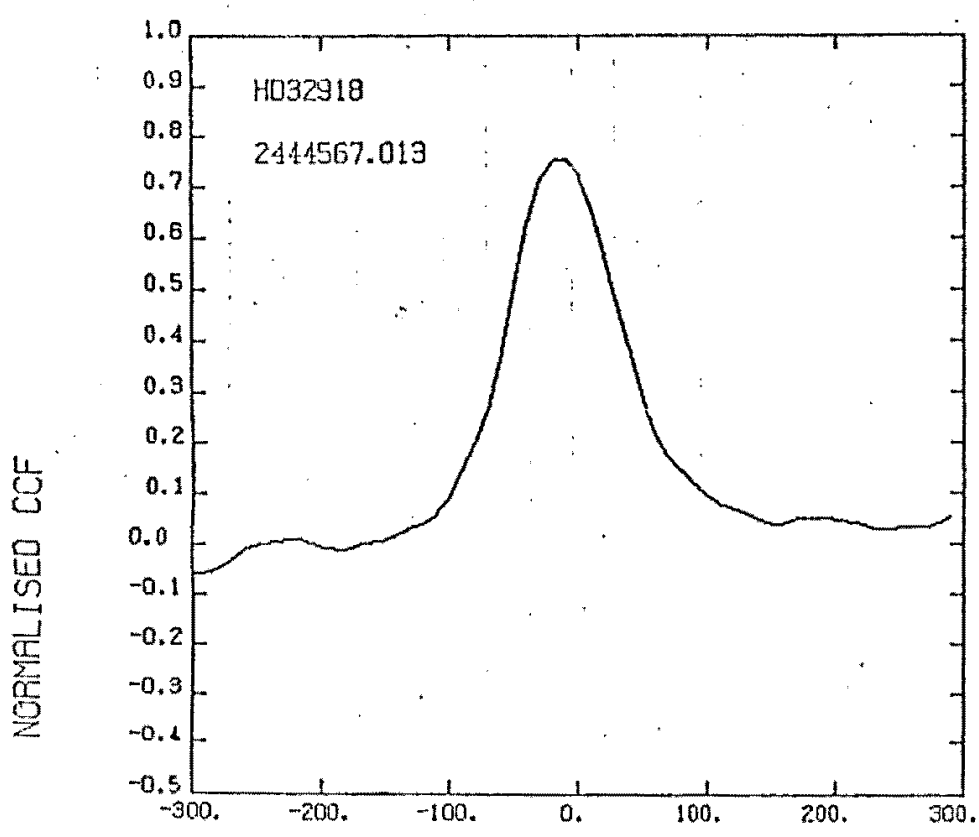

VELOCITY SHIFT $\mathrm{Km} / \mathrm{sec}$

(b)

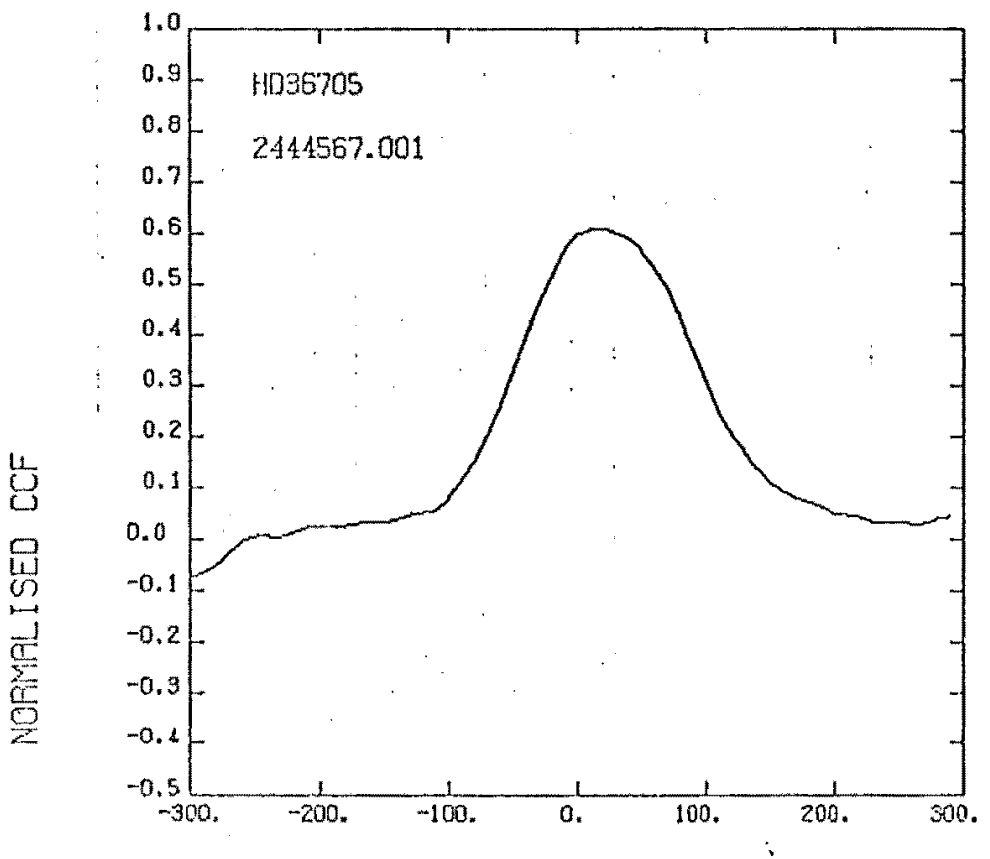

VELOCITY SHIFT $\mathrm{Km} / \mathrm{sec}$

(c)

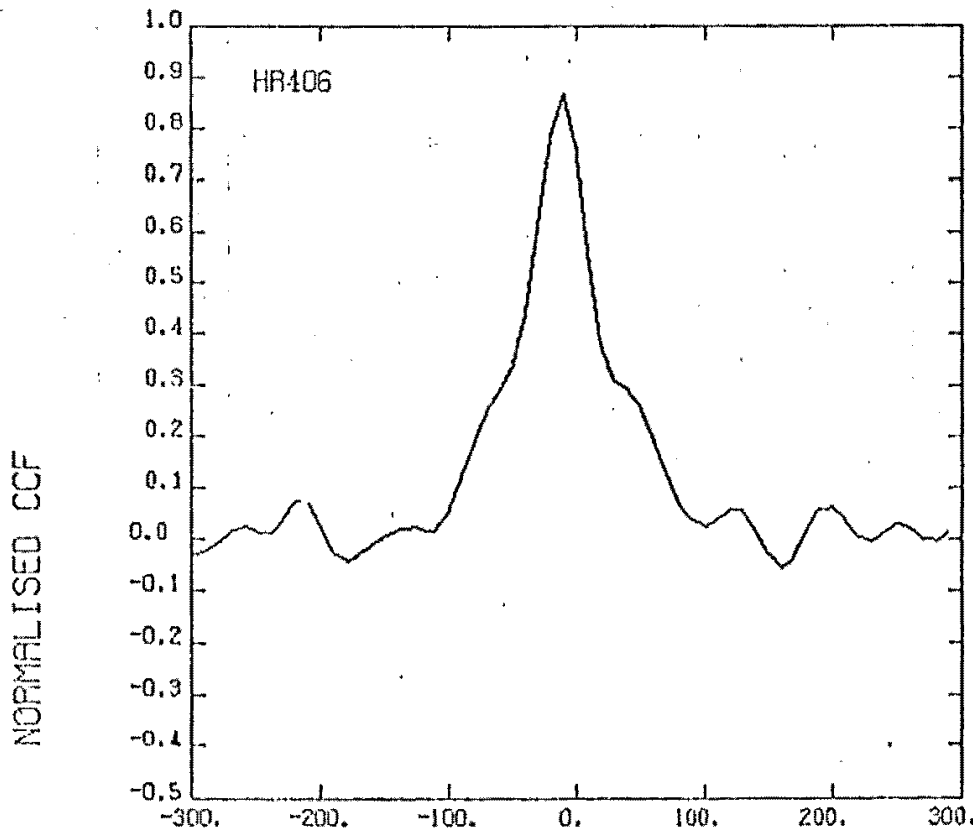

VELOCITY SHIFT Km/sec 
Figure 3 .

(a)

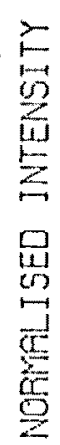

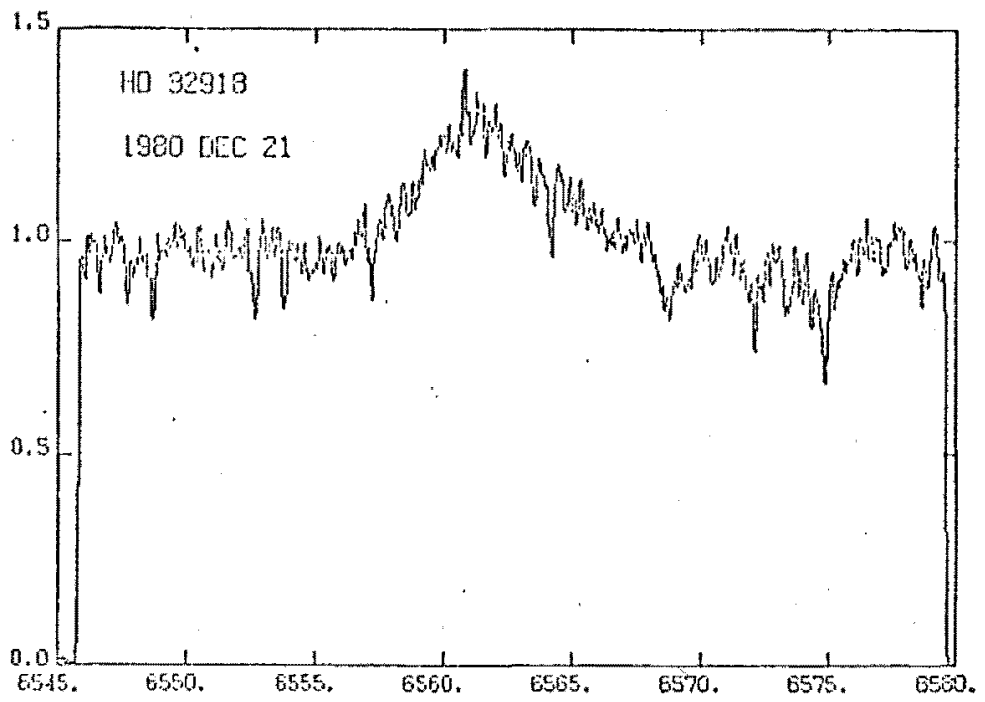

VAVELENGTH (A)

(b)

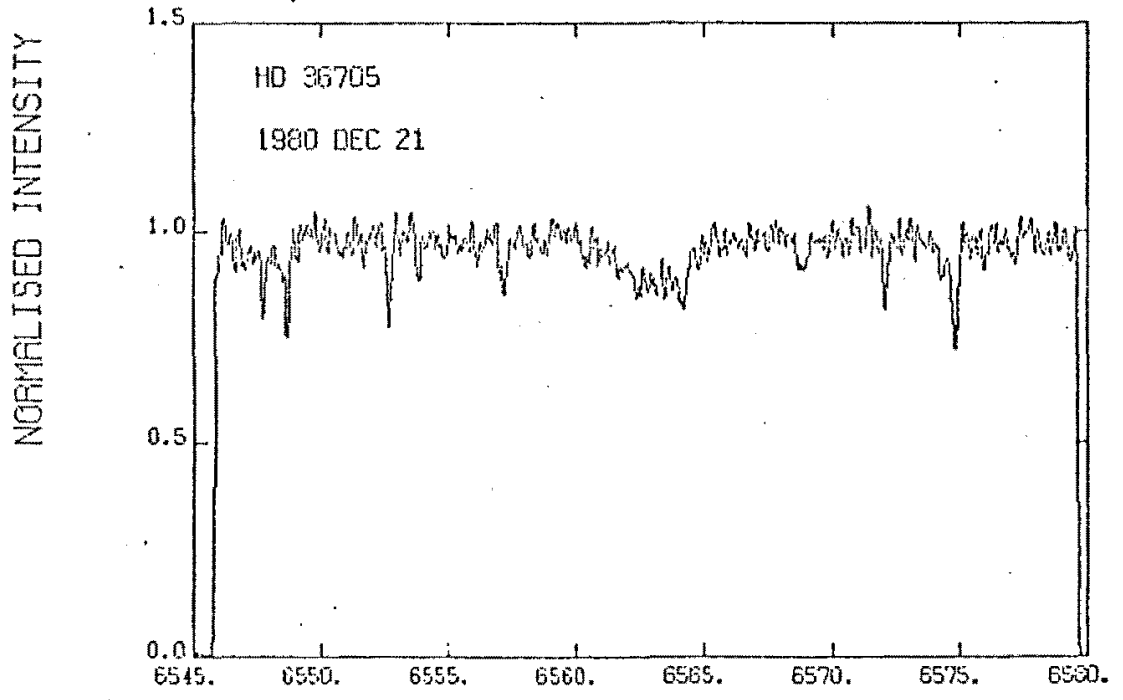

UAVELENOTH (A)

(c)

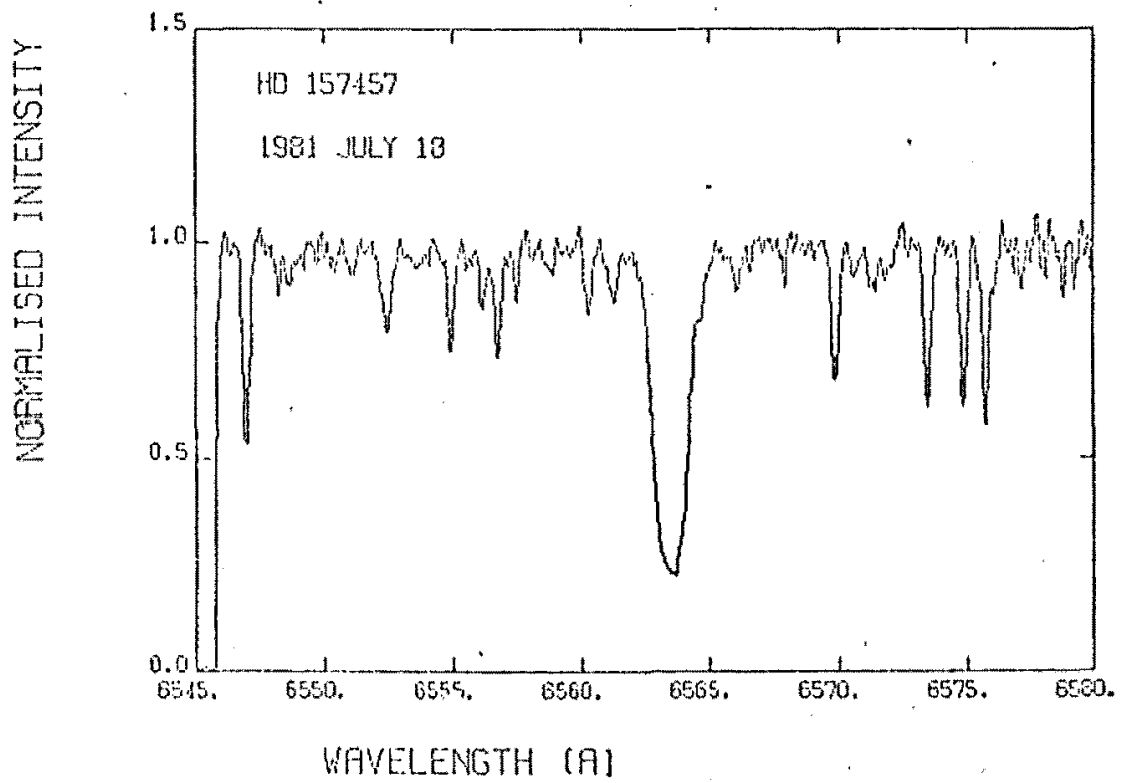


The work presented in this paper is based on an investigation which I initiated early in 1981, with the help of Dr.A.E.Wright of the CSIRO Radiophysics Division.

As principal investigator on the project, I prepared the source list, and was present at Parkes throughout the survey, sharing the tasks of observing and data reduction with the CSIRO co-authors. Mr. D.J. Hillier obtained the $\mathrm{H} \alpha$ spectra. I performed the reduction of these spectra at Mt. Stromlo, after the survey was completed, and prepared the first draft of the paper. Subsequent additions and alterations to the sections concerning the details of the radio observations have been made by the CSIRO co-authors.

\section{A. C. Collibr.}

A.C. Collier 
A COORDINATED RADIO AND Ha SURVEY OF SOUTHERN RS CVN SYSTEMS AND RELATED OBJECTS

A.C. Collier, Department of Physics, University of Canterbury, Christchurch, New Zealand.

R.F. Haynes, O.B. Slee and Alan E. Wright, CSIRO, Division of Radiophysics, P.O.Box 76, Epping, 2121, N.S.W., Australia.

and

D.J. Hillier, Mount Stromlo and Siding Spring Spring Observatories, Woden P.O., A.C.T., 2606, Australia. 
SUMMARY

We present the results of a search for radio emission at $5 \mathrm{GHz}$ from a selection of 21 southern hemisphere RS CVn binaries and related late-type emission-line stars. Three of the program stars were detected above the survey limit of $10 \mathrm{mJy}$, including the previously known RS CVn radio binary HR 1099 and two new radio binaries, HR 4492 (=HD 101379) and HD 195040. A simultaneous program of high-resolution $H \alpha$ spectroscopy revealed significant emission profile variability in both the latter stars. Radio variability on timescales of $\sim 1$ lay was observed in both HR 1099 and HR 4492. An increase by a factor of 3 in the observed radio flux density from HR 4492 on 1981 July 18 was accompanied by a significant enhancement of the Ho equivalent width by $\sim 0.5 \AA$ on that date. 


\section{INTRODUCTION}

Radio surveys of northern, close-binary stars in recent years have revealed the RS Canum Venaticorum (RS CVn) stars (Hal1, 1976; Eaton \& Hall, 1979) as an important class of flaring, apparently non-thermal radio sources at centimetre wavelengths (Owen \& Gibson, 1978). Recent unpublished work by A.C.Collier at optical wavelengths has resulted in the identification of sixteen new RS CVn binaries in the southern hemisphere.

The purposes of our survey were (a) to begin monitoring this sample of southern, active-chromosphere objects in a manner similar to that employed by several earlier northern radio surveys (Spangler, Owen \& Hulse, 1977), and (b) to explore the suspected link between variations in the observed radio flux density and the strength of the Ha emission from these stars.

Our survey included three objects which are not strictly members of the RS CVn class. Two of these are single stars of luminosity class III exhibiting extreme rotational brcadening ( $v_{e} \sin i \sim 70 \mathrm{~km} \mathrm{~s}^{-1}$ ), and strong emission features at $\mathrm{H} \alpha$ and the $\mathrm{Ca}$ II $\mathrm{H}+\mathrm{K}$ lines; both have been classified by Collier (1982a) as new members of the FK Comae class (Bopp \& Stencel, 1981). The third, HR 4492, is a quadruple system in which the active component appears to be an early $\mathrm{K}$ giant of mass $\sim 3 \mathrm{M}_{\odot}$ with a companion of mass $\sim 0.6 \mathrm{M}_{\odot}$ in a 54-day orbit. The $\mathrm{K}$ giant exhibits $\mathrm{H \alpha}$ and Ca II emiss. on comparable to that observed in the most active RS CVn systems (Collier, 1982b), and was the strongest new radio source 
detected during this survey.

We report here the results of an eight-day monitoring program carried out using the $5 \mathrm{GHz}$ receiver on the Parkes 64-metre telescope, and a simultaneous four-night $\mathrm{H} \alpha$ survey of the radio program stars with the $1.88 \mathrm{~m}$ telescope at Mt. Stromlo observatory.

\section{SOURCE SELECTION}

The program stars were selected according to the results of a series of photometric and spectroscopic surveys by A.C. Collier of late-type southern stars with strong chromospheric emission reversals in the $\mathrm{Ca}$ II $\mathrm{H} \& \mathrm{~K}$ line cores. The optical program stars were initially selected from the lists of Bidelman \& MacConnell (1973), Hearnshaw (1978) and Weiler \& Stencel (1978). Of the 21 stars selected for the radio survey, 17 show definite short-period radial velocity variations, 15 have high rotational smearing of their spectra, and 16 have abnormally shallow absorption and/or variable emissior features at $\mathrm{H} \alpha$. Spectroscopic orbits and/or photometric rotation periods have been determined for 12 of the stars surveyed.

III. INSTRUMENTATION AND OBSERVATIONAL TECHNIQUES

(a) Radio

The Parkes $64 \mathrm{~m}$ telescope was equiped for these measurements with a dual-feed system which produced two beams each of half-power width 4.3 arc min. One beam was located on-axis and the other was displaced off-axis by 
10.1 arc min on the sky.

Two different observing techniques were used. The first was "on-on" or wagging observations in which the telescope was moved so that the on-axis and then the offaxis beams observed the star alternately each 30 secs (see e.g. Wright, 1974). This method was used for the initial search since it is a fast way to detect weak sources. For those stars lying close to the galactic plane, background confusion can lead to erroneous detections (and non-detections). For those stars we suspected may have been so affected, we made extra wagging observations at four positions each 2.1 arc min from the star and forming a cross pattern around the star.

Objects for which a signal was detected by wagging were later scanned in both right ascension and declination to : (a) Further investigate the possibility of confusing sources in the star's neighbourhood; and (b) obtain a source position.

Such scans were typically each of length 40 arc min (about 10 half-power beamwidths) with individual data samples every 0.2 arc min.

\section{b) Optical}

The Ha spectroscopy was carried out using the ecchelle spectrograph at the coudé focus of the $1.88 \mathrm{~m}$ telescope at Mount stromlo observatory. The detector used was the 1024element red-sensitive photon colnting array (stapinski, Rodgers \& Ellis, 1979). The effective dispersion of this configuration was $2.7 \AA_{\mathrm{mm}^{-1}}$ or $0.034 \AA$ per reticon array element. A slit width of $200 \mu$ gave a resolution of $0.2 \AA$. 
The spectra were reduced using the SPECT spectral reduction package on the VAX $11 / 780$ computer at Mount Stromlo. Fixed pattern noise was removed by division of each spectrum by a "Flat-field" exposure of the spectrum of an incandescent source, made prior to the start of observations each night. A mild Gaussian filter was applied to the data prior to continuum fitting.

\section{OBSERVATIONAL RESULTS}

1. Radio survey

of the 21 stars surveyed, three detections were made above the $3 \sigma$ level of $10 \mathrm{mJy}$. A summary of the survey results is given in Table $I$.

HR 1099 was seen to exhibit variability on timescales of about a day, the flux density varying between 20 and 70 mJy. Further, there is little doubt that significant variation occurred in six hours, e.g. on July $17 / 18$ (see Fig. 1 (a)).

HD 195040 was detected at the $5 \sigma$ level at $16 \mathrm{mJY}$ on all days of the survey. Four positions located a half beamwidth (2.1 arc minutes) to each side of the source in both right ascension and declination confirmed that the source was compact. A series of 100,60-second scans each of length 40 arc min in both right ascension and declination gave a source position only 18 arc seconds north of the SAO catalogue position. Bearing in mind the weakness of the source and pointing uncertainties this indicates that the identification of the source with HD 195040 is probably correct. 
HR 4492 was detected as a $40 \mathrm{mJy}$ source initially. However, the source is located only $3^{\circ}$ south of the galactic plane. Scans of the region, as described above, revealed a compact radio source at the required optical position but there existed a small amount of confusion in the vicinity. The effect of one particular confusing source in the reference beam was eliminated by rotating the feed through $90^{\circ}$ and nodding, rather than wagging, to obtain flux measurements over the hour angle range concerned. The source position derived from the scans was

$$
\begin{aligned}
& \alpha_{1950}=j 1^{\mathrm{h}} 37^{\mathrm{m}} 05^{\mathrm{s}} \cdot 3 \quad \delta_{1950}=-65^{\circ} 07^{\prime} 31^{\prime \prime} . \\
& \text { The SAO position for HR } 4492 \text { is } \\
& \alpha_{1950}=11^{\mathrm{h}} 37^{\mathrm{m}} 09^{\mathrm{s}} \cdot 7 \quad \delta_{1950}=-65^{\circ} 07^{\prime} 14^{\prime \prime} .
\end{aligned}
$$

The discrepancy in these positions is probably not significant when pointing uncertainties and the weakness of the source are taken into account. The flux density was found to be variable (Fig.l(b)), with similar amplitude and timescale to the variation obsered in HR 1099, indicating that the physical size of the source is small.

A sharp increase by a factor of $\sim 3$ to a flux density of $65 \mathrm{mJy}$ was observed for HR 4492 on $1980 \mathrm{July} 18$; the level dropped again to $25 \mathrm{mJy}$ on July 19. The increase is similar to the flaring episodes observed previously in HR 1099 and a number $\mathrm{C}$ I other RS CVn binaries.

A frurth object, HD 36705, was detected at the 30 level on three days out of five. Observations at the half-power points of the on-axis beam, however, revealed the side effects of an extended source. The location of the source on the 
edge of the Large Magellanic Cloud casts considerable doubt on the validity of identifying it as the radio counterpart of HD 36705 .

2. $H \propto$ Observations

Fifty-two spectra were obtained on the nights of 1981 July 16-19 inclusive. The results are summarized in Table II. Following the radio detections of HR 4492 and $\mathrm{HD} 195040$, particular emphasis was laid on observing these two objects for time-dependent profile changes. The most obvious Ha variability was seen in the case of HD 195040, as illustrated in Figure 2. However, the low flux density of the radio source during our observations and the rather poor signal-to-noise ratio of the spectra did not permit any conclusions to be arawn concerning correlations between the two types of activity.

HR 4492 showed an extended Ha emission feature on all four nights, slightly displaced towards the blue part of the spectrum with a FWHM near $30 \mathrm{~km} \mathrm{~s}^{-1}$ and with an $H \alpha$ absorption core with the same radial velocity as the metallic line spectrum. The increase in radio flux density on July 18 was accompanied by an enhancement of the red wing of the emission feature.

In Figure 2, several photospheric absorption features are seer. to overlap with the extended wings of the $\mathrm{H} \alpha$ emission in $H R$ 4472. Accurateevaluation of the $H \alpha$ emission surface flux cannot be made without a detailed model atmosphere analysis of the underlying $H \alpha$ absorption profile, and an accurate spectrophotometric flux calibration. 
Instead, a method was adopted which permitted variations in the equivalent width of the $\mathrm{H} \alpha$ emission to be measured accurately, and which would allow a surface flux calibration to be made when the required information becomes available. A "total equivalent width" for all absorption and emission features, over the wavelength interval $6551.70 \AA$ to $6572.80 \AA$ was evaluated in the rest frame of the star for each spectrum, using

$$
W(\AA)=\int_{6551.7}^{6572.8} \frac{F(\lambda)-F_{c}(\lambda)}{F_{c}(\lambda)} d \lambda
$$

where $F_{C}(\lambda)$ is the fitted monochromatic continuum flux level and $F(\lambda)$ is the observed flux, in arbitrary units. The sign convention adopted in equation (1) is such that the contribution of the emission is positive, and those of the photospheric absorption features are negative.

The results are plotted in Figure 3. The total equivalent widths over the same wavelength range for the radial velocity standard star HD 109379 (= B CrV spectral type G5 III) are also plotted, and give some idea of the expected night-to-night scatter in the results.

An increase of $0.34 \pm 0.12 \AA$ in the emission width of HR 4492 occurred between July 17 and July 18. Although only one spectrum was obtained on July 19, a decrease of similax magnitude in the emission width from the previous night i. evident. 
V. DISCUSSION

The detection of HR 4492 as a flaring radio source resembling $H R 1099$ in its quiescent state, adds to the body of evidence for a very high level of chromospheric and coronal activity in this system. It has been examined in the ultra-violet (Stacy, Stencel \& Weiler, 1978) and has been identified as a source of both hard and soft X-rays (Garcia et al., 1980).

Houk and Cowley (1975) classify it as a composite-spectrum object, with pectral types $\mathrm{G} 5$ and $\mathrm{AO} ;$ those two components form a visual binary of separation $0.24 \pm 0.02$ arc seconds and undetermined period (Worley, 1978). The AO component is itself an eclipsing binary, with components near AOV and A2V. The chromospherically-active component is the other member of the visual pair. The spectral type is $\mathrm{K} 4$. III, and the mass and radius are in the range $2.5 \pm 0.5 \mathrm{M}_{\odot}$ and $40 \pm 10 \mathrm{R}_{\odot}$ respectively. It is a single-lined spectroscopic binary, with a companion whose mass probably lies in the range $2.0 \pm 0.5 \mathrm{M}_{\odot}$, and an orbital period of $53.9 \pm 0.1$ days (Collier, 1982b).

Despite the dominance of the earlier components' spectrum in the blue, strong emission is seen in the $H \& K$ lines of $\mathrm{Ca}$ II sharing the radial velocity variations of the $\mathrm{K}$ giant and with a similar degree of rotational broadening, $v_{e} \sin i=10 \pm 2 \mathrm{~km} \mathrm{~s}^{-1}$. The timescale for tidal synchronization of the giant's convective envelope (zahn, 1977) is of the order of $10^{4}$ years, indicating that the giant should rotate synchronously with its companion. The 
observed degree of rotational broadening is consistent with this.

The distance of the system is $140 \pm 30 \mathrm{pc}$, assuming typical main sequence luminosities for the A stars in the shorter-period binary.

The angular diameter of the $\mathrm{K}$ giant, derived from the estimated $V-R$ colour index using the Barnes-Evans relationship (Barnes, Evans \& Moffett, 1978) is $(2.5 \pm 0.2) \times 10^{-3}$ arc sec. The brightness temperature thus ob'aining during the flare-like event on $1981 \mathrm{July} 18$ is

$$
\begin{aligned}
& B^{2} \mathrm{~T}_{\mathrm{b}}=(7.7 \pm 2.0) \times 10^{8} \mathrm{~K} \\
& \text { where } \beta \text { is the ratio } \frac{\text { source diameter }}{\text { stellar diameter }}
\end{aligned}
$$

Thermal emission from a $10^{7} \mathrm{~K}$ corona would require a source diameter some nine times the stellar diameter, or roughly seven times the 54 -day binary separation.

It is unlikely that the radio emission can come from a stellar wind region of similar size and temperature. The relatively low value of the velocity of such a wind $(200 \mathrm{~km}$ $\sec ^{-1}$ - deduced from the Ha emission width) would imply a minimum timescale for significant radio variability of about 25 days as compared with the observed variability which has timescales of the order of 1 day or less. The simultaneous enhancement observed at both $\mathrm{H \alpha}$ and at $5 \mathrm{GHz}$ on July 18 suggests that a non-thermal mechanism similar to that postulated for HR 1099 (Owen \& Gibson, 1978) is more likely to be responsible. Further similtane us radio observations of this object at different frequencies are needed to determine its radio spectral index. 
The detection of HD 195040 was unexpected, in that the apparent visual magnitude of this system is only $\mathrm{V}=9.0 . \quad$ The brightness temperature obtained from the observed $5 \mathrm{GHz}$ flux density and the Barnes-Evans angular diameter of $0.38 \mathrm{milliarc}$ sec is

$$
B^{2} \mathrm{~T}_{\mathrm{b}}=(7 \pm 3) \times 10^{9} \mathrm{~K}
$$

This figure is comparable with that which may be derived by the same method from the flux densities observed at various frequencies in HR 1099 during its 1978 February outburst (Feldman et al., 1978). The strong, variable $\mathrm{H} \alpha$ emission profiles observed during the survey support the conecture that a large flaring episode was in progress during the survey. Further observations to establish the degree of variability of the radio source should therefore be made.

\section{ACKNOWLEDGMENTS}

We would like to thank the Director and staff at Mount Stromlo and siding spring observatories, for the generous allocations of telescope and computer time which made the optical side of this project possible. 
REFERENCES

Barnes, T.G., Evans, D.S. and Moffett, T.J., 1978. Mon.Not.R.Astron.Soc, , 183, 298.

Bidelman, W.P. and MacConnell, D.J., 1973. Astron. J.,78, 687. Bopp, B.W. and stencel, R.E., 1981. Astrophys.J.(Lett.), 247, L131.

Collier, A.C. 1982a. Mon.Not.R.Astron.Soc. (submitted). 1982b. Ph.D. thesis, University of Canterbury (in preparation).

Eaton, J.A. and Hall, D.S., 1979. Astrophys. J., 227, 907. Feldman, P.A., et al., 1978. Astron. J., 83, 1471. Garcia, M., et al., 1980. Astrophys. J. (Lett.), 240, L107. Hall, D.S. 1976. IAU Coll. $29,287$.

Hearnshaw, J.B., 1979. IAU Col1. 46, 371. Houk, N. and Cowley, A.P., 1975. Michigan Speotral Catalogue, Vol. I, University of Michigan. Houk, N., 1978. Michigan Spectral Catalogue, Vol. II, University of Michigan.

Owen, F.N. and Gibson, D.M., 1978. Astron. J., 83, 1488. Spangler, S.R., Owen, F.N. and Hulse, R.A., 1977. Astron.J., 82, 989 .

Stacy, J.G., Stencel, R.E. and Weiler, E.J., 1980. Astron. J., 85, 858 .

Stapinski, T.E., Rodgers, A.W. and Ellis, M.J., 1979. Advances in Eleotronics and EZeotron Physics, 52, 389. Weiler, E.J. and Stencel, R.E., 1978. Astron. J., 84, 1372. Worley, C.E., 1978. Publications of the U.S. Naval observatory, Vol. XXIV, Part VI, 89. 
Wright, A.E., 1974. Mon. Not. R. Astron. Soc., 167, 251. Zahn, J.P., 1977. Astron. Astrophys., 57, 383 . 
TABLE I

Summary of Parkes $5 \mathrm{GHz}$ survey results

\begin{tabular}{|c|c|c|c|c|c|}
\hline $\begin{array}{c}\text { Object } \\
\text { HD }\end{array}$ & $\begin{array}{l}\text { Dist. } \\
\text { (pc) }\end{array}$ & $\begin{array}{c}\text { No.of } \\
\text { days } \\
\text { examined }\end{array}$ & Detection & $\begin{array}{c}\text { Flux } \\
\text { Density } \\
(\mathrm{mJy})\end{array}$ & Notes \\
\hline 5303 & $54 *$ & 4 & no & & \\
\hline 8435 & $(100)$ & 3 & no & & \\
\hline 14643 & $77 *$ & 4 & no & & \\
\hline 22468 & 35 & 7 & yes & $25-70$ & $=\mathrm{HR} 1099$ \\
\hline 32918 & $\geqslant 300 *$ & 5 & no & & FK Comae type \\
\hline 36705 & $40 *$ & 5 & possible & $\leqslant 10$ & FK Comae type \\
\hline 37434 & $(30)$ & 4 & no & & $=\mathrm{HR} 1927$ \\
\hline 37847 & $(50)$ & 3 & no & & \\
\hline 46697 & $(60)$ & 6 & no & & \\
\hline 77137 & 50 & 6 & no & & \\
\hline 81410 & $38 *$ & 5 & no & & \\
\hline 101309 & $62^{*}$ & 7 & no & & \\
\hline 101379 & $140 *$ & 8 & yes & $10-65$ & $=\mathrm{HR} 4492$ \\
\hline 119285 & $(80)$ & 1 & $?$ & & Confused regio \\
\hline 127535 & $63 *$ & 1 & $?$ & & Confused regio \\
\hline 137164 & $54 *$ & 6 & no & & \\
\hline 155555 & $30 *$ & 5 & no & & \\
\hline 174429 & $(75)$ & 5 & no & & \\
\hline 181809 & $31 *$ & 4 & no & & \\
\hline 195040 & $99 *$ & 7 & yes & 15 & \\
\hline 196818 & $53 *$ & 5 & no & & \\
\hline
\end{tabular}

Distances in parentheses estimated from visual magnitude, assuming $\mathrm{M}_{\mathrm{V}} \sim+3.5$ for a typical RS CVn subgiant component. Asterisked distances from Barnes-Evans angular diameters estimated system dimensions (Collier, 1981b). 
TABLE II

\begin{tabular}{|c|c|c|c|c|c|}
\hline ct & sp. type & $\mathrm{V}_{\max }$ & $\begin{array}{l}\text { No. of } \\
\text { Spectra }\end{array}$ & $\begin{array}{c}\mathrm{H} \alpha \\
\text { Profiles }\end{array}$ & $\begin{array}{l}\text { Velocity } \\
\text { variations }\end{array}$ \\
\hline 03 & $\mathrm{~F} 8 \mathrm{~V}+\mathrm{K} l \mathrm{IV}$ & 7.5 & 1 & Filled & SB2 \\
\hline 68 & $\mathrm{G} 8 \mathrm{IV} / \mathrm{V}+\mathrm{K} I \mathrm{IV}$ & 6.0 & 1 & Emission & SB2 \\
\hline 09 & $\mathrm{G} 5 \mathrm{~V}+\mathrm{K} 1 \mathrm{IV}$ & 8.0 & 1 & Emission & SB2 \\
\hline 79 & $\mathrm{~K} 2-4 \operatorname{III}(+\mathrm{A} 0)$ & 4.8 & 15 & $\begin{array}{l}\text { Emission }+ \\
\text { abs.core }\end{array}$ & SBI \\
\hline 79 & G5 III & 2.7 & 5 & Absorption & RV standar \\
\hline 85 & *KI Vp & 8.0 & 1 & $\begin{array}{l}\text { Emission }+ \\
\text { abs.core }\end{array}$ & SBI \\
\hline 35 & Kl IV & 8.3 & 1 & Emission & SBI \\
\hline 64 & $\mathrm{~K} 2 \mathrm{IV}$ & 7.0 & 2 & Shallow abs. & SB2 \\
\hline 55 & $\mathrm{G} 8 \mathrm{~V}+\mathrm{K} 0 I \mathrm{~V}$ & 6.8 & 3 & Filled & SB2 \\
\hline 57 & G8 III & 5.2 & 3 & Absorption & RV standar \\
\hline 29 & $* \mathrm{~K} 0 \mathrm{Vp}$ & 7.9 & 1 & $\begin{array}{l}\text { Broad, } \\
\text { shallow abs. }\end{array}$ & - \\
\hline 09 & $\mathrm{~K} \perp \mathrm{IV}$ & 6.8 & 1 & Absorption & - \\
\hline 40 & K3 III/IV & 8.4 & 7 & $\begin{array}{l}\text { Variable em; } \\
\text { abs.core }\end{array}$ & SBI \\
\hline 18 & $\mathrm{~K} 3 \mathrm{III} / \mathrm{IV}$ & 7.6 & 1 & Shallow abs. & - \\
\hline 38 & K0 III & 5.8 & 2 & Abs. & stande \\
\hline
\end{tabular}

ectral types from Houk and Cowley (1975); Houk (1978). 


\section{FIGURE CAPTIONS :}

Fig. 1. $5 \mathrm{GHz}$ flux densities for (a) HR 1099 and (b) HR 4492, plotted versus time. Each point represents the mean value of four consecutive wags; a $1 \sigma$ error bar is given near the left hand margin of each plot.

Fig. 2. Ha profiles of stars detected at parkes, illustrating observed night-to-night profile changes in HD 195040 and HD 101379 (=HR 4492). An Ha spectrum of HR 1099 is also included for comparison. Note increase in red wing of the HD 101379 emission on 1981 July 18.

Fig.3. Total equivalent widths in $\mathrm{H} \alpha$ region plotted versus time for HD 101379 (dots) and the radial velocity standard stars HD 109379 (Sp. type G5 III ; squares), HD 157457 (G8 III; open circles) and HD 203638 (KO III ; crosses). The standard error of the observations is indicated by the error bar at left. 

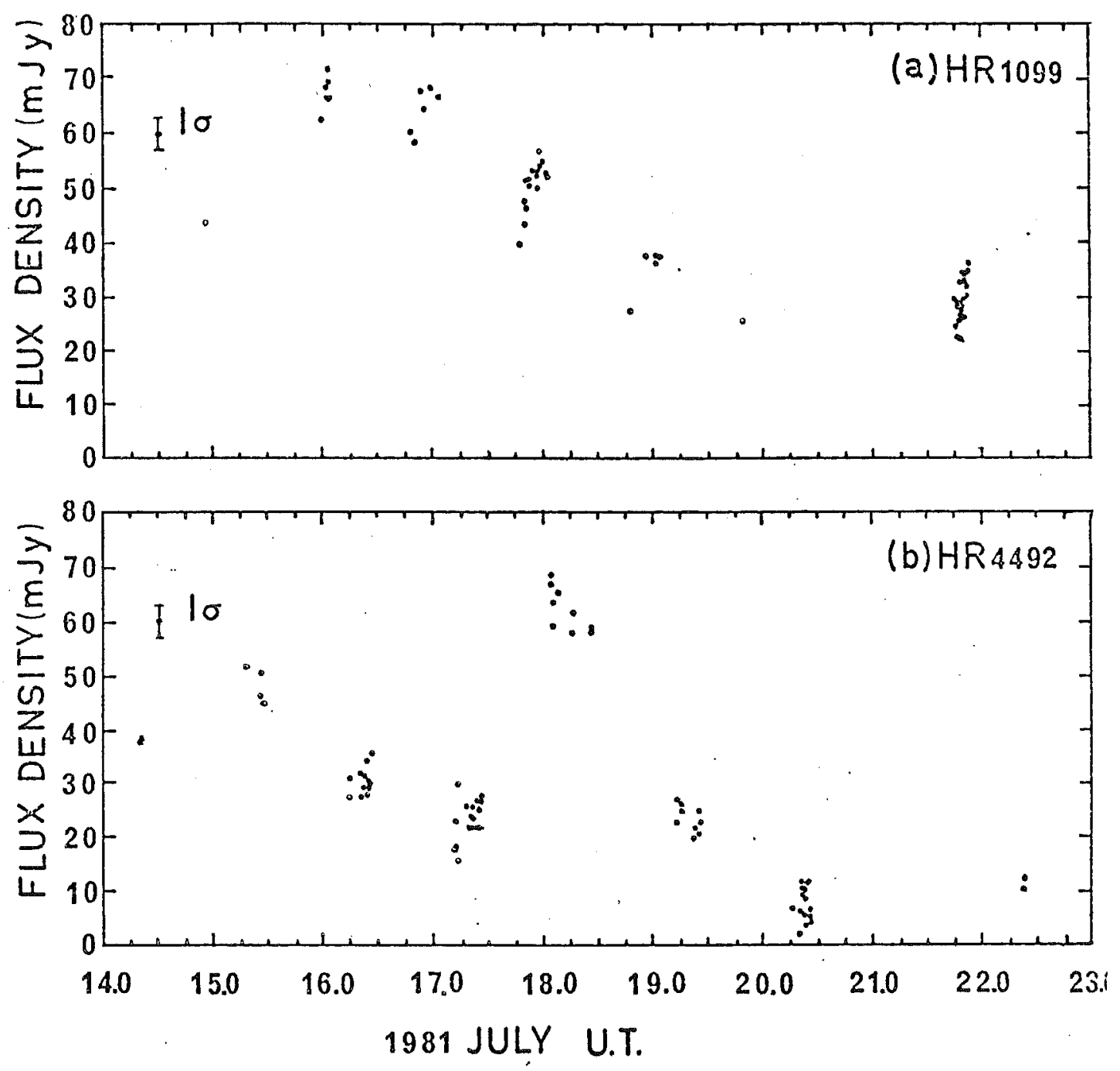

Figure 1. 
Figure 2.
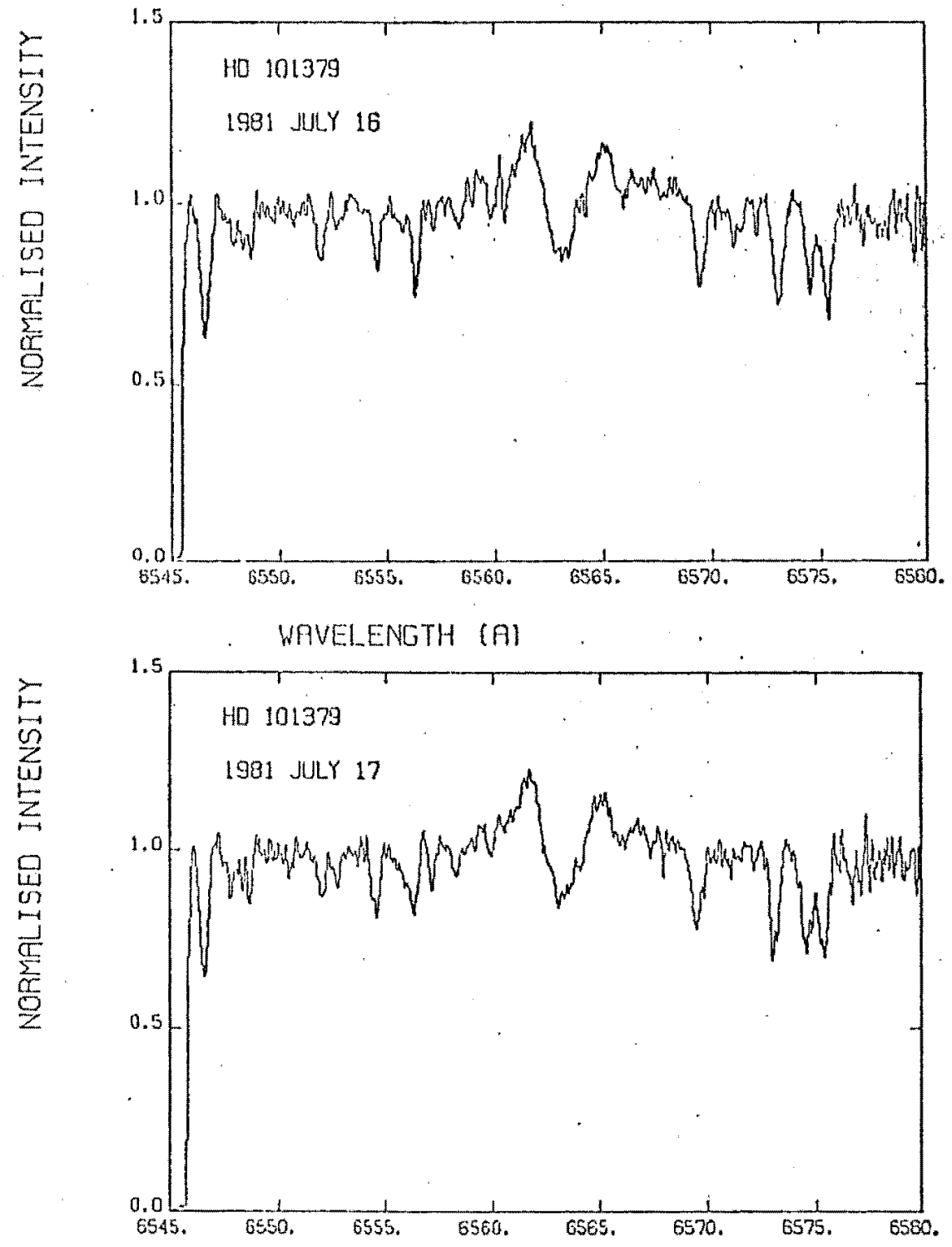

WAVELENGTH (A)

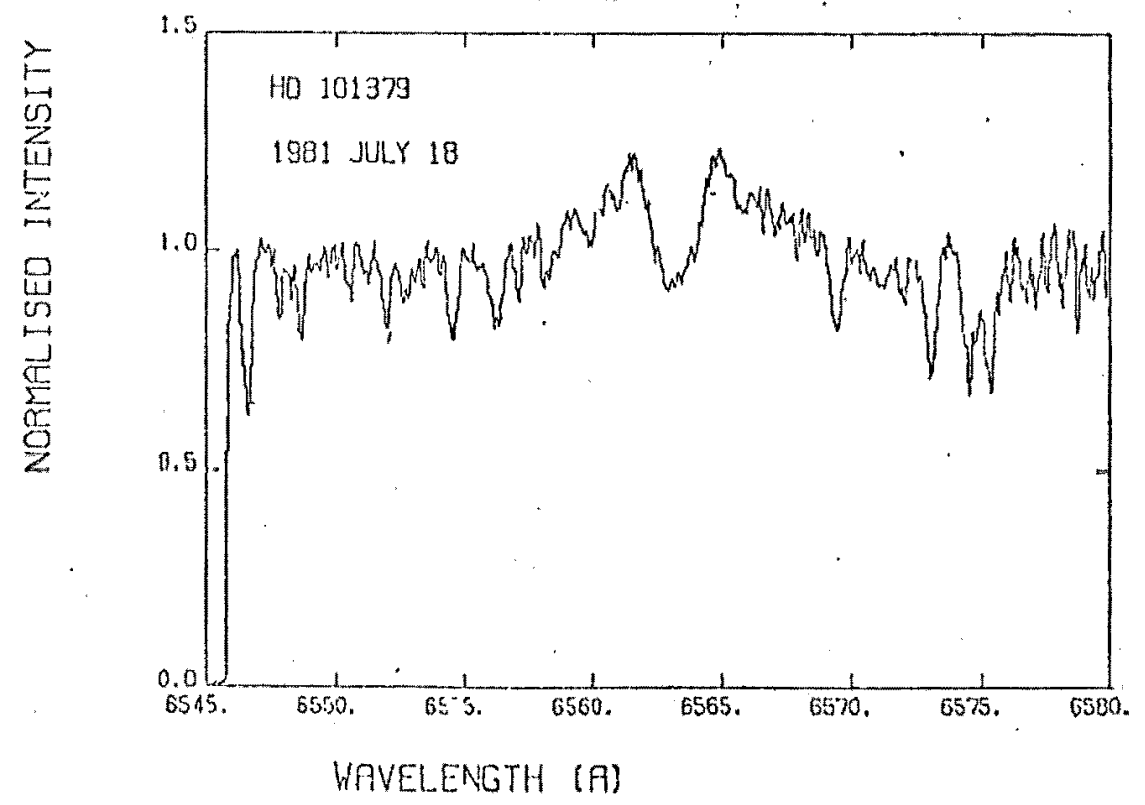


Figure 2 cont...

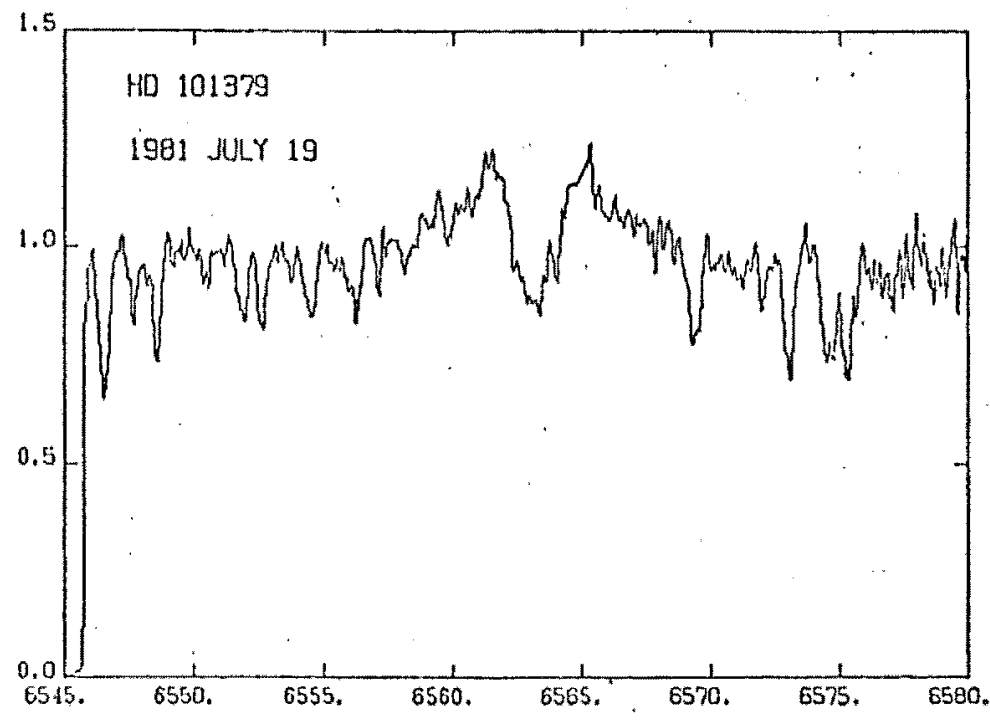

WAVELENGTH (A)
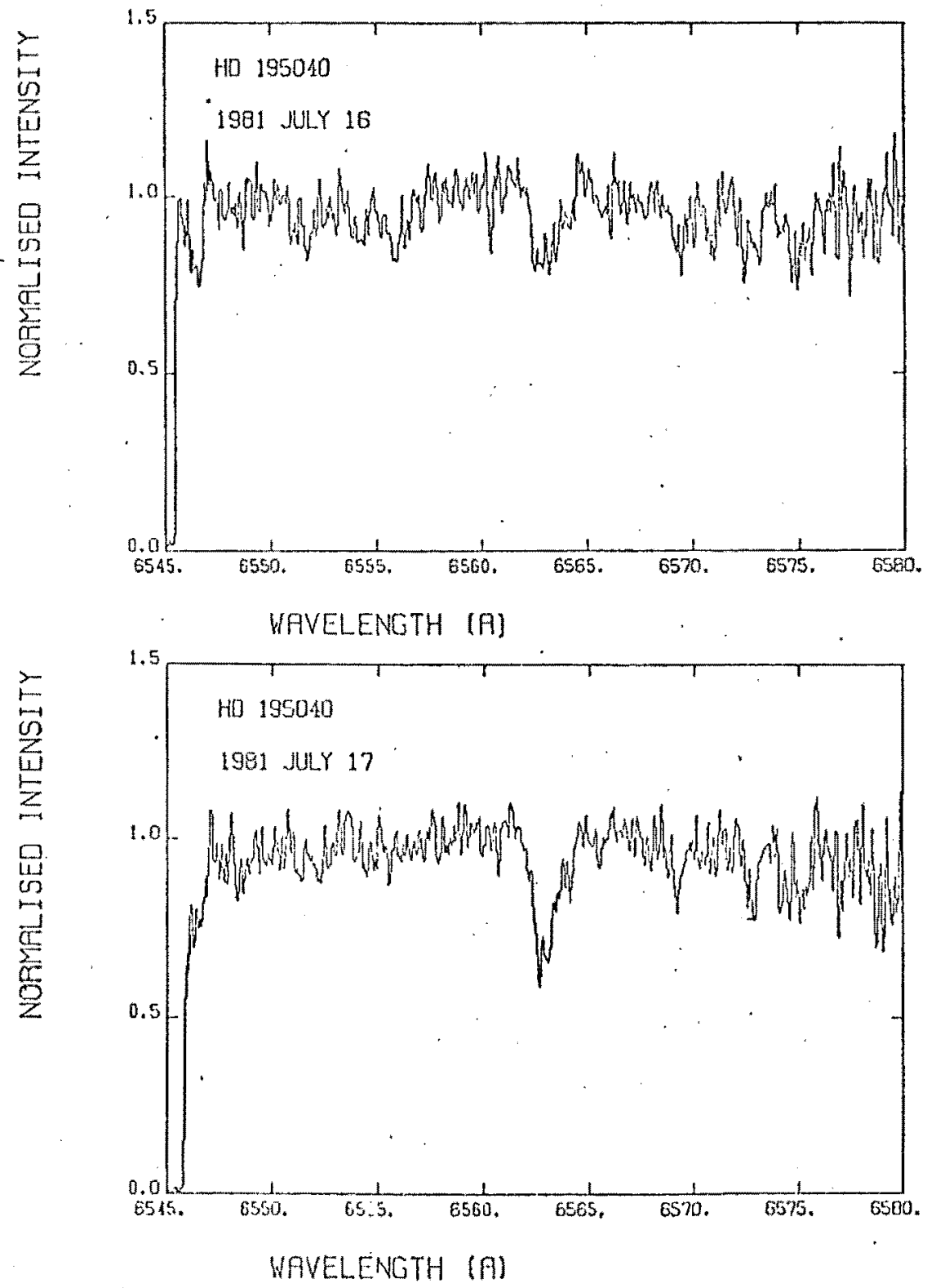
Figure 2 cont,..

告

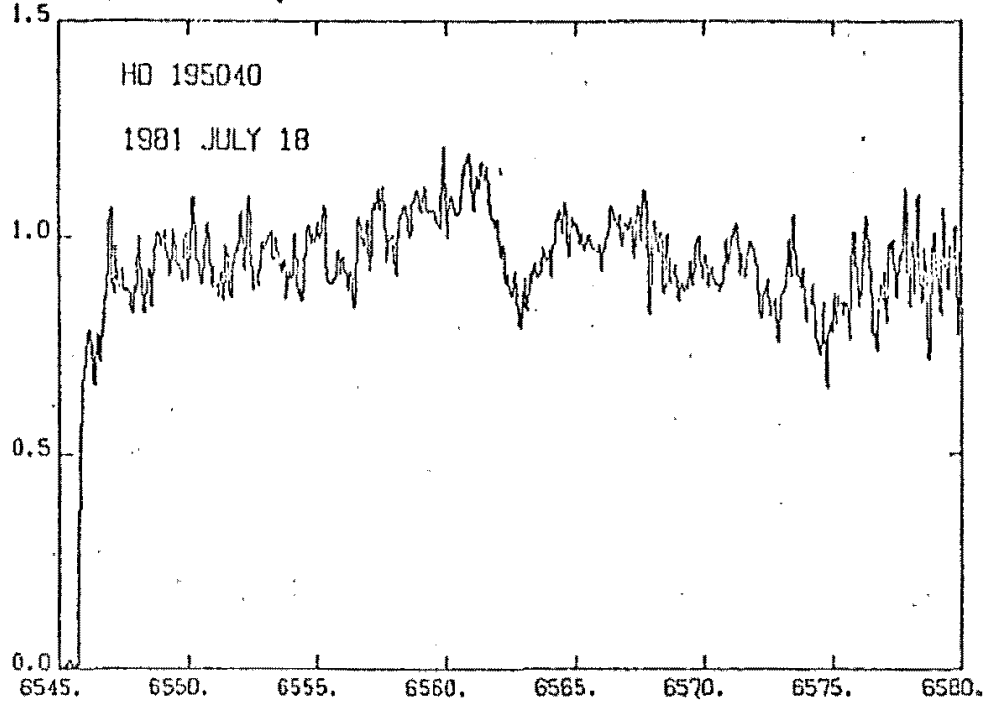

WAVELENGTH (A)

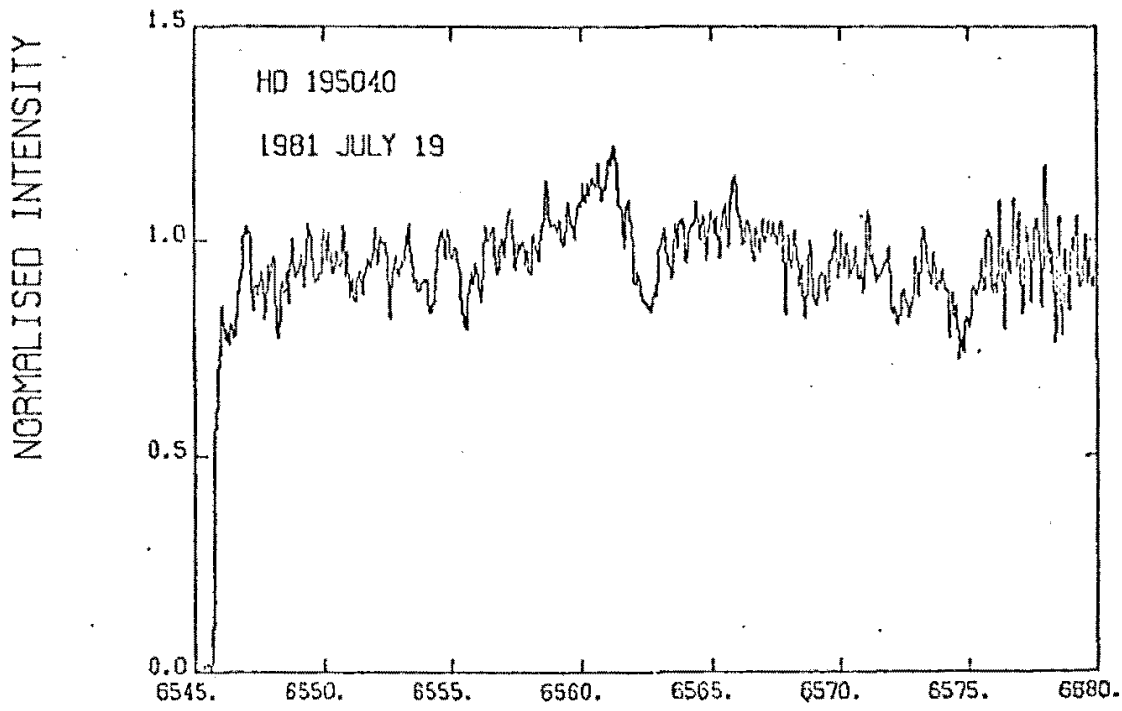

VAVELENGTH (A)

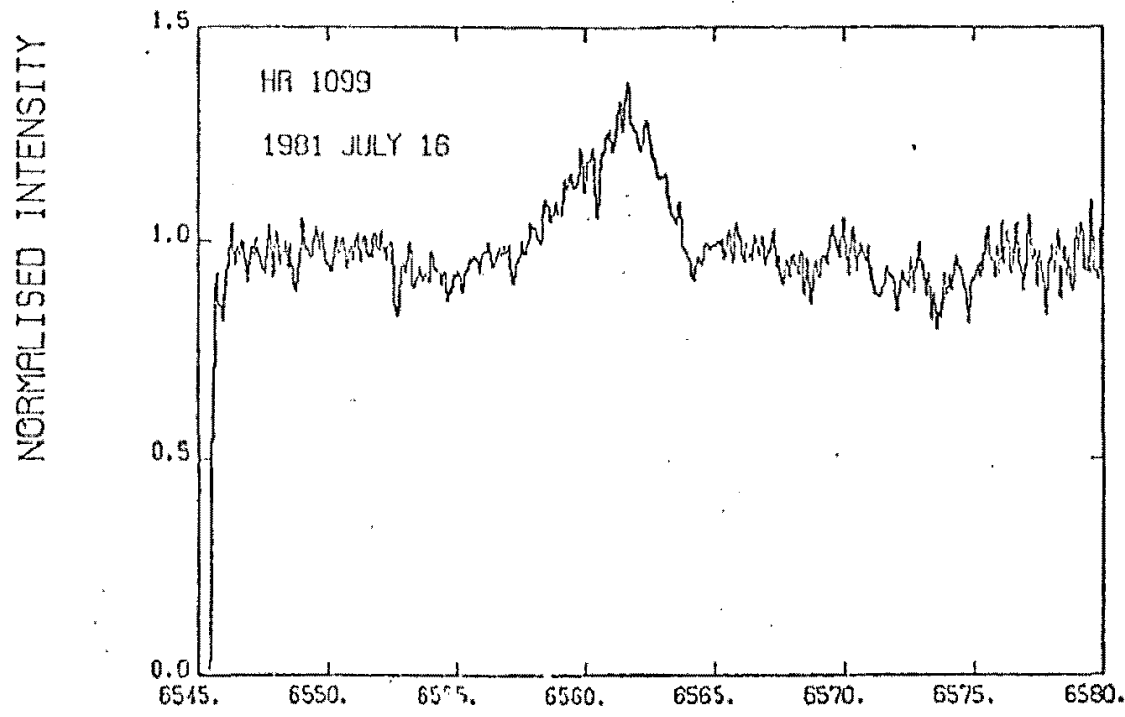

WAVELENGTH (A) 


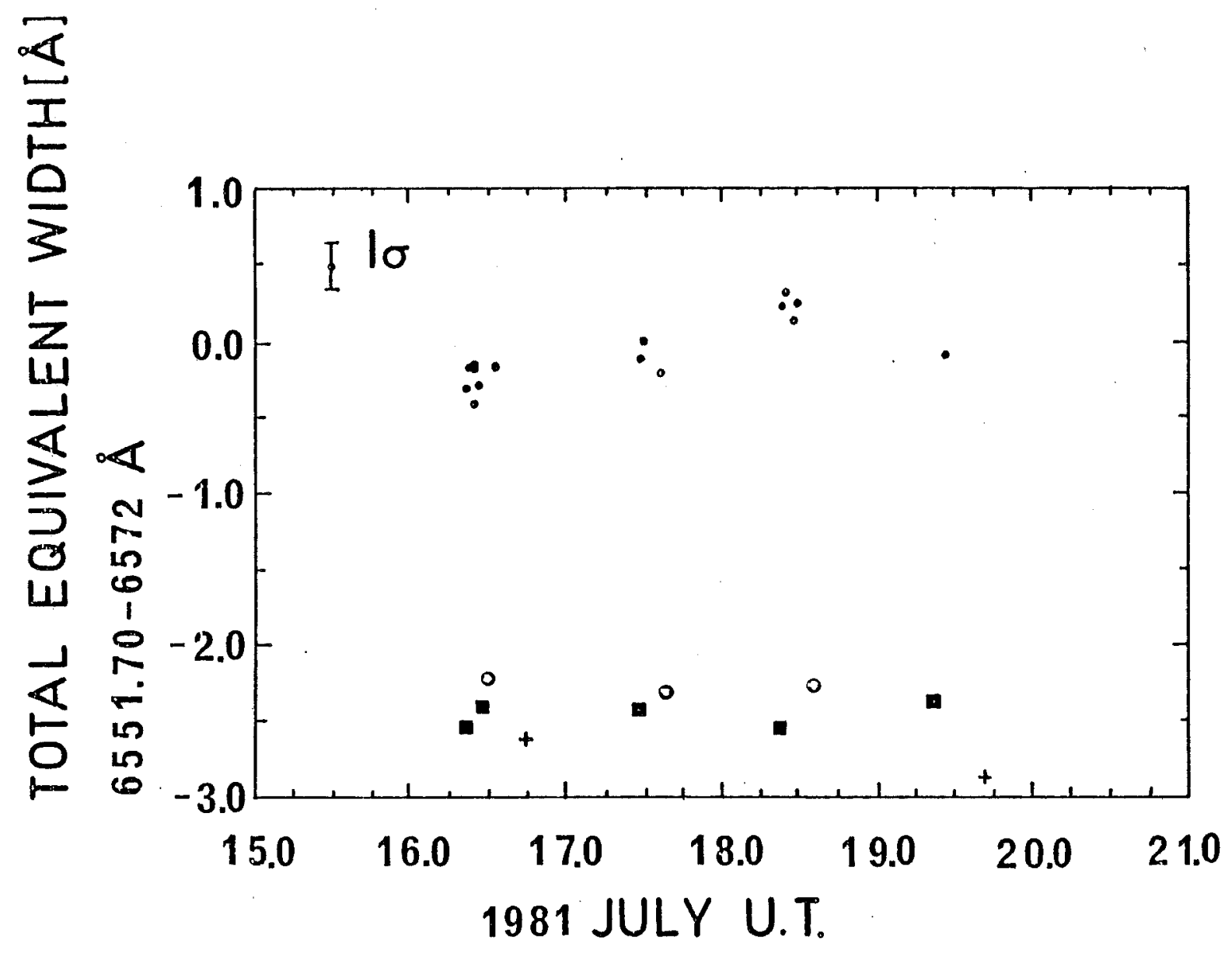

Figure 3 . 